INTERNATIONAL MONETARY FUND

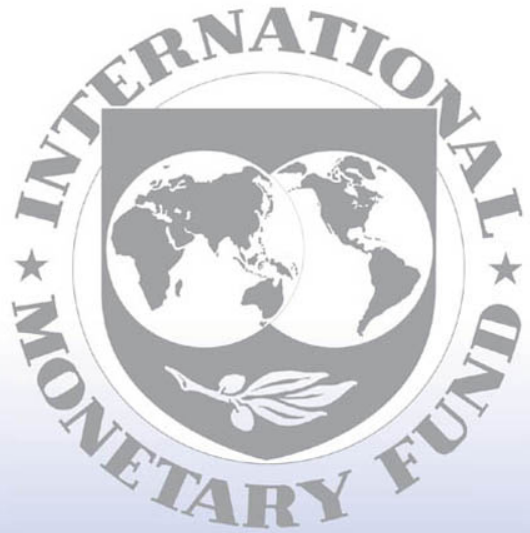

Staff

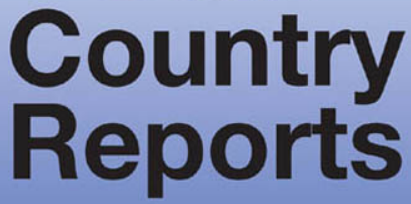




\section{Democratic Republic of the Congo: Staff Report for the 2009 Article IV Consultation, Request for a Three-Year Arrangement Under the Poverty Reduction and Growth Facility, and Request for Additional Interim Assistance Under the Enhanced Initiative for Heavily Indebted Poor Countries}

The following documents have been released and are included in this package:

- $\quad$ The staff report, prepared by a staff team of the IMF, following discussions that ended on Friday, December 11, 2009, with the officials of the Democratic Republic of the Congo on economic developments and policies. Based on information available at the time of these discussions, the staff report was completed on November 30, 2009. The views expressed in the staff report are those of the staff team and do not necessarily reflect the views of the Executive Board of the IMF.

- $\quad$ Supplements to the staff report.

- $\quad$ A Public Information Notice (PIN).

- $\quad$ A Press Release summarizing the views of the Executive Board as expressed during its December 11, 2009 discussion of the staff report that completed the request and review.

- $\quad$ A statement by the Executive Director for the Democratic Republic of the Congo.

The document(s) listed below will be separately released.

Letter of Intent sent to the IMF by the authorities of the Democratic Republic of the Congo * Memorandum of Economic and Financial Policies by the authorities of the Democratic Republic of the Congo * Technical Memorandum of Understanding* Other Background papers

*Also included in Staff Report

The policy of publication of staff reports and other documents allows for the deletion of market-sensitive information.

Copies of this report are available to the public from

International Monetary Fund • Publication Services

$70019^{\text {th }}$ Street, N.W. • Washington, D.C. 20431

Telephone: (202) 623-7430 • Telefax: (202) 623-7201

E-mail: publications@imf.org Internet: http://www.imf.org

\section{International Monetary Fund Washington, D.C.}


INTERNATIONAL MONETARY FUND

DEMOCRATIC REPUBLIC OF THE CONGO

\title{
Staff Report for the 2009 Article IV Consultation, Request for a Three-Year Arrangement Under the Poverty Reduction and Growth Facility, and Request for Additional Interim Assistance Under the Enhanced Initiative for Heavily Indebted Poor Countries
}

\author{
Prepared by the African Department \\ (In consultation with other departments) \\ Approved by Mark Plant and Dominique Desruelle
}

November 30, 2009

Mission dates: March 16-31; April 22-28; August 10-18; and October 27-November 10, 2009.

Team: Messrs. Ames (head), Ben Ltaifa, and Farah, and Ms. Bovha-Padilla (all AFR) and Messrs. Callegari (FAD), Hostland (SPR), and Jahjah (Resident Representative). The team coordinated closely with the local World Bank office.

Context: Growth in 2008 was strong, but was weakened by the onset of the global financial crisis during the second half of the year that resulted in a deterioration of the country's terms of trade and large job losses in the mining sector. The escalation of the conflict in the eastern provinces, along with weaknesses in public financial management, resulted in large spending overruns during the fourth quarter of 2008, which placed pressure on the exchange rate and inflation through early 2009 . The constitutionally mandated fiscal decentralization policy created further challenges during the year given weak institutional capacity at both the central and provincial levels. Macroeconomic policies for the rest of 2009 and 2010 aim at reducing inflation while mitigating the impact of the global financial sector on the economy.

Article IV consultation: Discussions focused on policies and reforms that (i) foster sustained and higher economic growth; (ii) enhance external sustainability; and (iii) strengthen institutional capacity for economic management.

Poverty Reduction and Growth Facility (PRGF): The authorities are requesting a new three-year arrangement under the PRGF in an amount of SDR 346.45 million (65 percent of quota) and additional interim assistance under the enhanced Heavily Indebted Poor Countries (HIPC) Initiative.

IMF relations: The last Article IV consultation took place on September 5, 2007, and since then staff have been monitoring the authorities' economic program through a series of staff monitored programs (SMPs). The previous PRGF arrangement (SDR 580 million; 109 percent of quota) was almost fully drawn (SDR 553.47 million) before it expired in March 2006 without completing the sixth review owing to policy slippages in the lead-up to the first democratic elections in four decades. The Democratic Republic of the Congo (DRC) reached the decision point of the enhanced HIPC Initiative in July 2003.

Exchange rate regime: The DRC's exchange rate regime is classified as a free float. It has accepted the obligations under Article VIII, section 2, 3, and 4 of the Fund's Articles of Agreement, but maintains an exchange restriction and a multiple currency practice. 
Acronyms

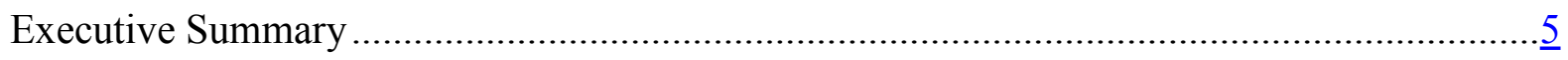

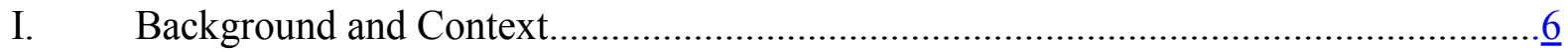

II. Recent Economic Developments ............................................................

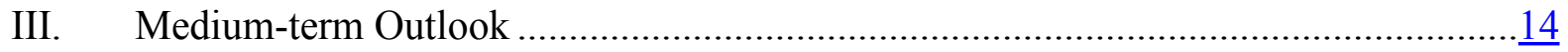

IV. Article IV Consultation and Policy Discussions............................................... 15

A. Fostering Sustained and High Economic Growth....................................... $\frac{15}{16}$

B. Enhancing External Sustainability ........................................................ $\frac{16}{18}$

C. Strengthening Institutional Capacity for Economic Management ......................18

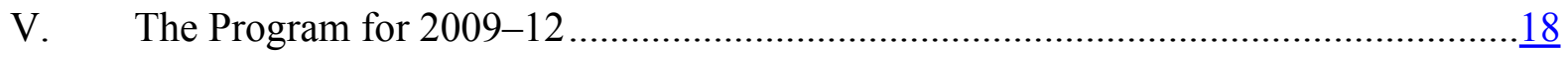

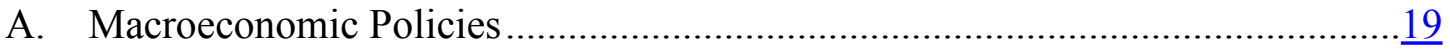

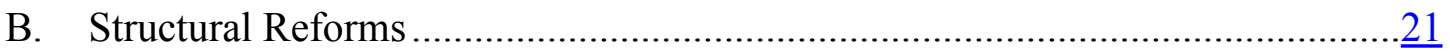

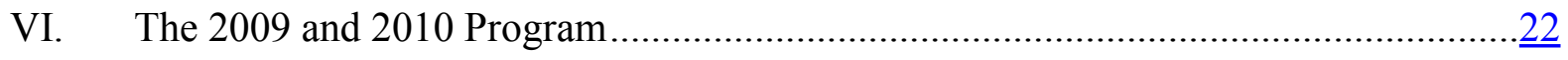

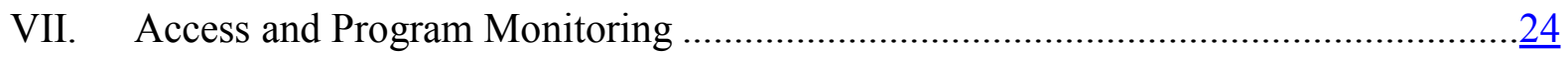

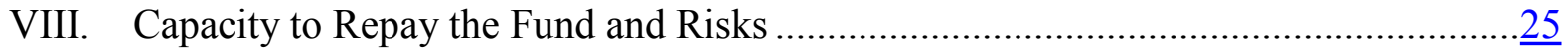

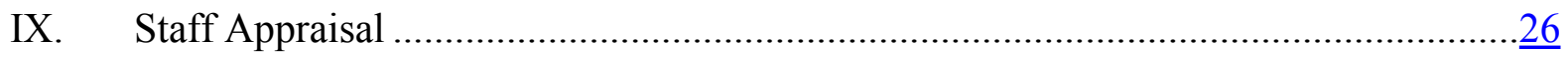

Boxes

1. The Sino-Congolese Cooperation Agreement …................................................10

2. Main Recommendations of the 2007 Article IV Consultations .................................. $\frac{15}{17}$

3. An Assessment of the Exchange Rate Policy and External Competitiveness ..............17

4. Poverty Reduction and Growth Strategy ................................................................19

Figures

1a. Progress Toward Millennium Development Goals 1990-2015 ...............................

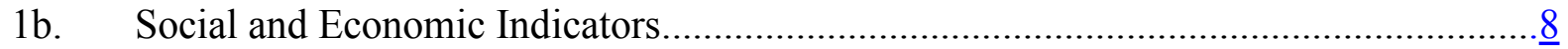

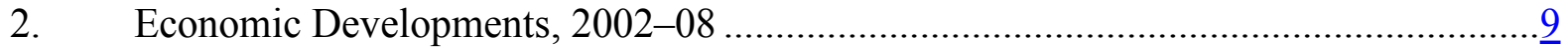

3. Recent Economic Developments …....................................................................

Text Tables

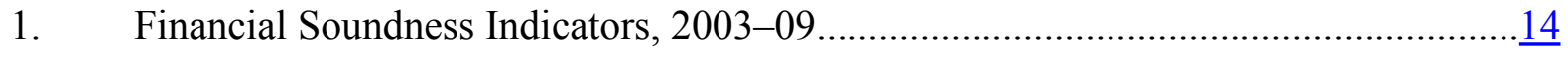

2. Structural Reform and Burden Sharing........................................................... 
Tables

1. Selected Economic and Financial Indicators, 2007-14 .........................................28

2a. Central Government Financial Operations, 2007-12 ........................................... $\frac{29}{30}$

2b. Central Government Financial Operations, 2007-12 ........................................... $\underline{\underline{30}}$

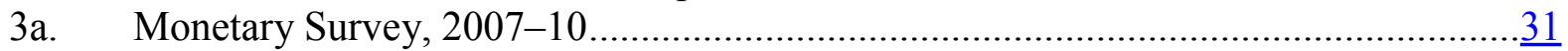

3b. Accounts of the Central Bank of the Congo, 2007-10 …......................................... $\frac{32}{33}$

4. Balance of Payments Summary, 2007-14 .............................................................33

5. Tentative Schedule of Disbursements and Reviews Under the

PRGF Arrangement, 2009-12 ....................................................................... $\frac{34}{35}$

6. Indicators of Capacity to Repay the Fund, 2009-22 ........................................... $\frac{35}{36}$

7. Progress Status of HIPC Completion Point Triggers.............................................

Appendix I

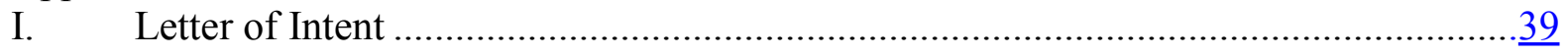

Appendix I Attachments

I. Memorandum of Economic and Financial Policies, 2009-12 ….................... $\frac{40}{62}$

II. Technical Memorandum of Understanding .............................................

Appendix I Tables

I.1. Quarterly Quantitative Performance Criteria and Indicative Targets, 2009-10 ..........54

I.2. Structural Conditionality and Macroeconomic Relevance, $2009-10$....................... $\frac{55}{56}$

I.3. Matrix of Economic and Financial Policy Measures for 2009-12 .......................... $\underline{56}$ 


\section{ACRONYMS}

AfDB

AFRITAC

ANAPI

$\mathrm{BCC}$

BTR

CEEAC

CIRR

DRC

DGRAD

DSA

HIPC

FDI

I-PRGS

JSAN

MCM

MDRI

MEFP

NCG

NDA

NEER

NFA

OCC

OFIDA

OHADA

PAP

PEG

PFM

PRGF

PRGS

PRS-NSC

PRSP

PV

RAC-ESF

REER

SADC

SB

SCCA

SMP

TA

TMU

VAT

African Development Bank

African Regional Technical Assistance Centers

National Investment Promotion Agency

Central Bank of the Congo

Central bank bills

Economic Community of Central African States

Commercial interest reference rate

Democratic Republic of the Congo

Directorate of Administrative and State Revenue

Debt Sustainability Analysis

Heavily Indebted Poor Countries

Foreign direct investment

Interim Poverty Reduction and Growth Strategy

Joint Staff Advisory Note

Monetary and Capital Markets Department

Multilateral Debt Relief Initiative

Memorandum of Economic and Financial Policies

Net credit to government

Net domestic assets

Nominal effective exchange rate

Net foreign assets

Congolese Office of Controls

Custom and Excise Collection Agency

Organization for the Harmonization of Business Law in Africa

Priority Action Plan

Government Economic Program

Public financial management

Poverty Reduction and Growth Facility

Poverty Reduction and Growth Strategy

Poverty Reduction Strategy National Steering Commission

Poverty Reduction Strategy Paper

Present value

Rapid Access Component of the Exogenous Shocks Facility

Real effective exchange rate

Southern African Development Community

Structural benchmark

Sino-Congolese Cooperation Agreement

Staff Monitored Program

Technical assistance

Memorandum of Technical Understanding

Value added tax 


\section{EXECUTIVE SUMMARY}

Recent developments. A sharp decline in world commodity prices in late 2008 slowed economic activity in 2009, while the escalation of conflict in the eastern provinces led to higher security spending, increased government borrowing from the central bank, and an upsurge in inflation and depreciation of the exchange rate during the first quarter of 2009. Lower government borrowing from the central bank and tight monetary policy during the first half of 2009 helped reduce inflation and stabilize the exchange rate. However, inflation increased and the exchange rate depreciated during the third quarter of 2009 reflecting excess liquidity in the economy largely due to central bank lending to distressed banks and failure to anticipate and mop up liquidity injections by the government. The DRC's debt situation remains unsustainable absent substantial debt relief.

Article IV Consultation. The discussions focused on fostering sustained and high economic growth, enhancing external sustainability, and strengthening institutional capacity for economic management. Key themes included:

- Enhancing macroeconomic stability. Fiscal policy should be geared to avoiding government recourse to central bank financing to alleviate the burden on monetary policy for achieving the inflation objective. Central bank reorganization, restructuring and recapitalization is key to enhancing its independence and capacity to conduct monetary policy. The free floating exchange rate regime is appropriate given the economy's susceptibility to exogenous shocks.

- Increasing fiscal space. The challenge is to mobilize domestic revenue and strengthen public financial management (PFM) in support of priority expenditure, including reconstruction. This requires bold reforms to modernize and consolidate tax administration, streamline exemptions, and simplify the tax system. Strengthening PFM, including at the provincial level given the decentralization policy, will also help improve expenditure allocation and mobilize development aid.

- Improving the business climate. The authorities need to press ahead with reforms to reduce the cost of doing business, with emphasis on restructuring public enterprises that provide growth critical services and on simplifying procedures for operating a business.

- Strengthening institutional capacity. The authorities need to strengthen policy coordination within government, including through the merging of the ministries in charge of finance and budget. Improving socioeconomic statistics is also critical for monitoring and assessing the effectiveness of policies and reforms.

New program. The authorities are requesting a three-year PRGF arrangement to support the implementation of their poverty reduction and growth strategy (PRGS) and additional interim HIPC assistance in the amount of SDR 45.66 million. The program aims to enhance macroeconomic stability, create fiscal space for priority spending, and accelerate structural reforms.

Program risks. The economy is vulnerable to exogenous shocks and the lingering conflict. Weak institutional capacity could also adversely affect program implementation. These risks could be lowered by increased donor financial and technical assistance and by the ongoing peace efforts to pacify the eastern provinces. 


\section{BACKGROUND AND CONTEXT}

1. Socioeconomic conditions in the DRC remain dire following a decade long conflict. The human cost and destruction of the country's social and economic infrastructure were enormous. As a result, although rich in natural resources, the country's per capita income and human development indicators remain amongst the lowest in Africa (Figure 1).

2. The return of peace to most of the country in $\mathbf{2 0 0 3}$ paved the way for political and economic stability. During 2003-06, the transitional government implemented prudent macroeconomic polices, restored confidence, brought hyperinflation under control, and laid the foundation for high levels of growth (Figure 2). This facilitated the installation of the new democratically elected government in March 2006.

3. The country's dilapidated infrastructure constitutes one of the principal impediments to economic growth, private sector development, and poverty reduction. Given the huge infrastructure development needs and limited concessional resources available from traditional development partners, the authorities are pursuing nontraditional modes of financing to fill the gap, including resource-backed infrastructure development financing (Box 1).

4. The security situation in the eastern provinces remains fragile despite recent peacekeeping efforts. Attacks by armed militants continue to destabilize the eastern provinces. In 2008 and early 2009, this resulted in urgent spending requests outside the expenditure chain that placed pressure on scarce budgetary resources. In March 2009, the government and key rebel groups signed an agreement to integrate combatants into the national army. Disarmament and reintegration remains a key challenge with important budgetary implications.

\section{The constitutionally mandated decentralization policy also poses significant} budgetary and implementation challenges. The authorities are committed to transferring 40 percent of government revenues to the provinces, along with the responsibility for implementing social sector programs. Given existing constraints, however, they have decided to slow down the pace of implementation while strengthening capacity in PFM and project implementation at the provincial level.

6. The onset of the global financial crisis has aggravated economic conditions. The sudden and sharp drop in world commodity prices resulted in a marked deterioration in the country's terms of trade - in the context of its narrow commodity export base - and a decline in foreign direct investment (FDI). This slowed economic activity, raised unemployment, and amplified macroeconomic imbalances. It also weakened fiscal performance, on top of an already low level of domestic revenue mobilization.

7. The DRC is in debt distress. The stock of external debt is about US\$13.1 billion with debt service amounting to one-fourth of total expenditure. At end 2008, the present value of public and publicly guaranteed external debt is estimated at 93 percent of GDP, 150 percent of exports, and 502 percent of government revenue (excluding grants), well above the policy-based threshold levels. ${ }^{1}$ Given resource constraints, the country continues to accumulate arrears to

\footnotetext{
${ }^{1}$ See the joint Fund/Bank Debt Sustainability Analysis.
} 
bilateral (including Paris Club) and commercial creditors, but remains current on obligations to international financial institutions. The authorities continue to seek a settlement with the London Club and other bilateral creditors on outstanding loans. The World Bank is financing, through its Debt Reduction Facility, financial and legal technical assistance to the DRC to reach a settlement with its London Club creditors.

Figure 1a. Democratic Republic of the Congo: Progress Toward Millennium Development Goals, 1990-2015

(Percent, unless otherwise indicated)
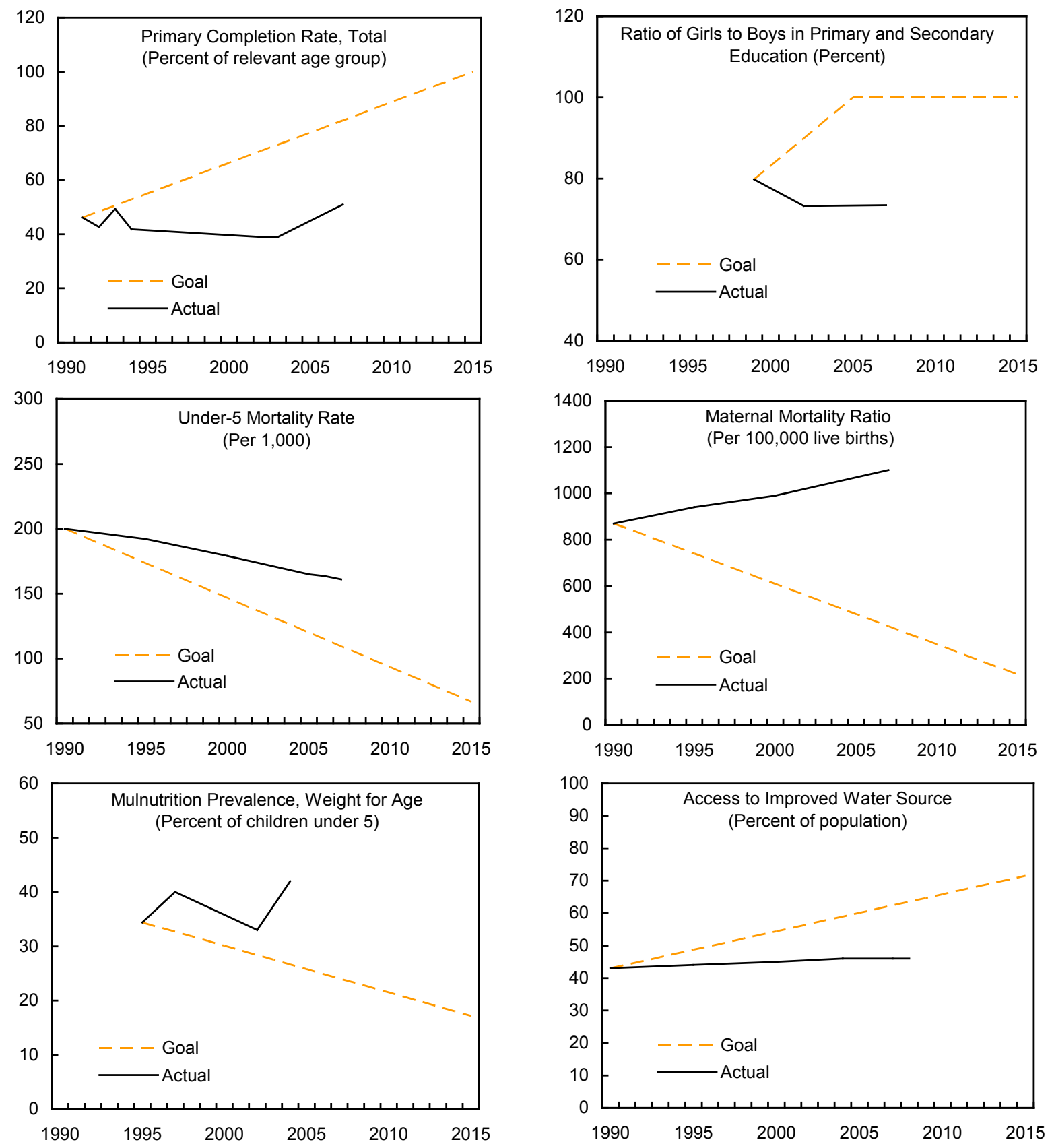

Sources: World Bank, http://ddp-ext.worldbank.org; and United Nations, http://unstats.un.org. 
Figure 1b. Democratic Republic of the Congo: Social and Economic Indicators
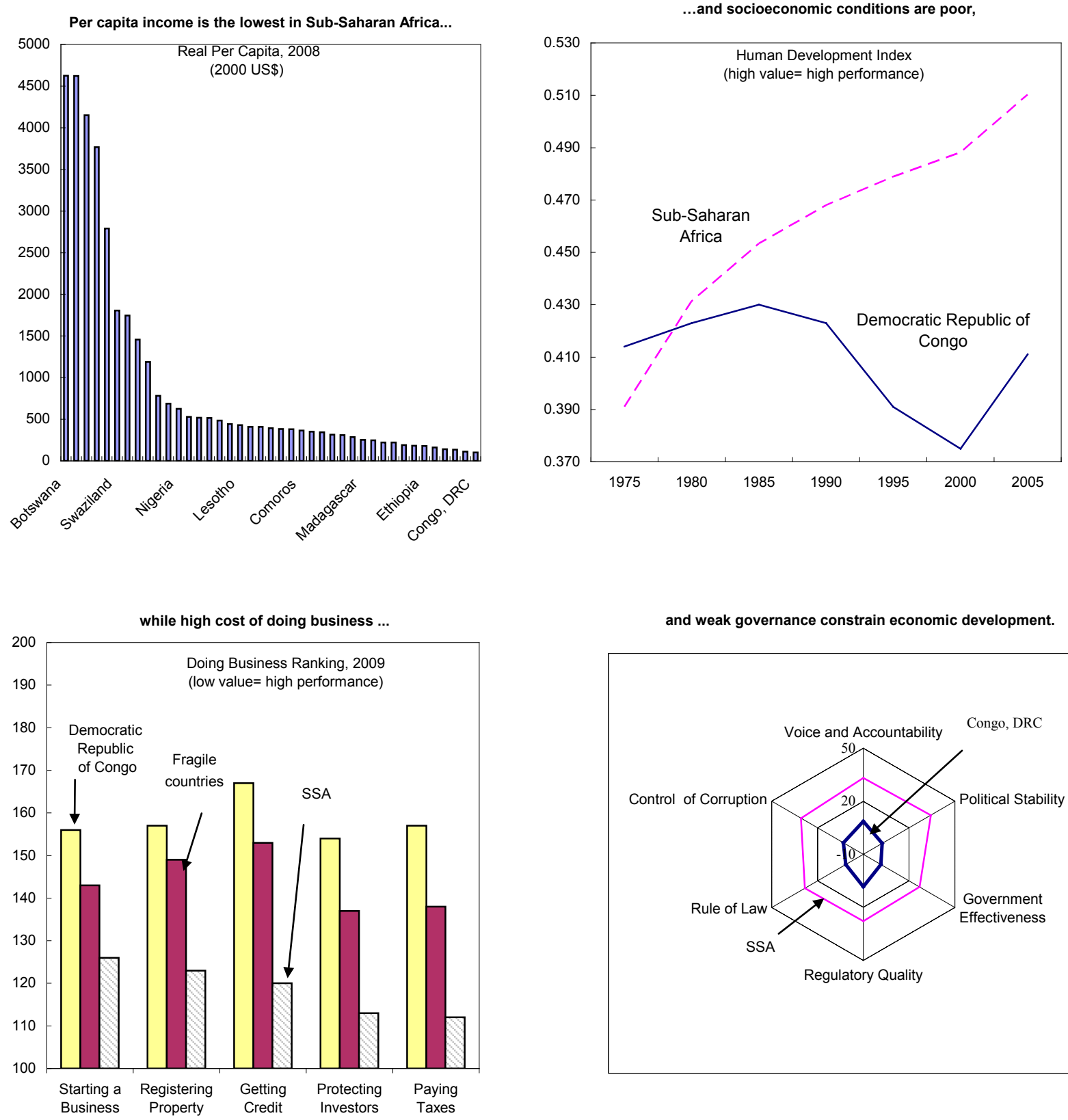

and weak governance constrain economic development.

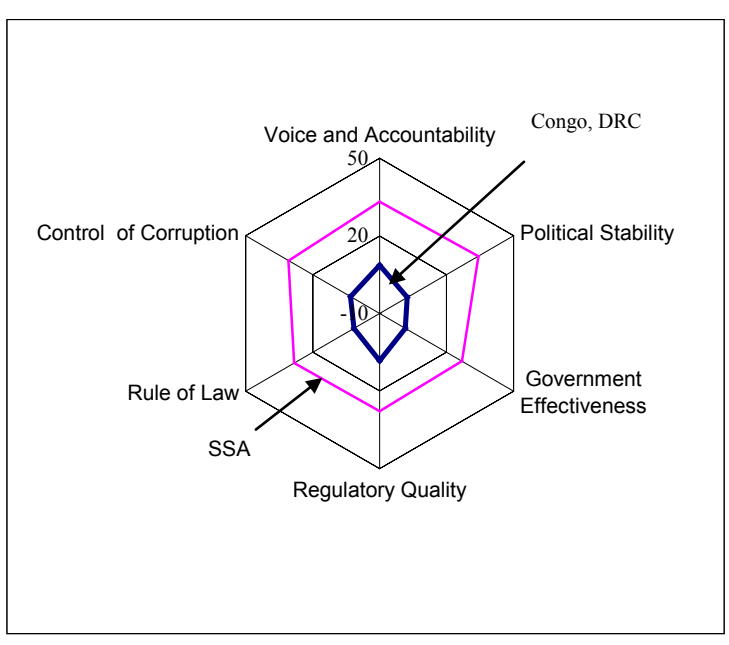

Sources: IMF, World Economic Outlook (2009), World Bank, Doing Business (2010), and Human Development Report, 2008-09. 
Figure 2. Democratic Republic of the Congo: Economic Developments, 2002-08
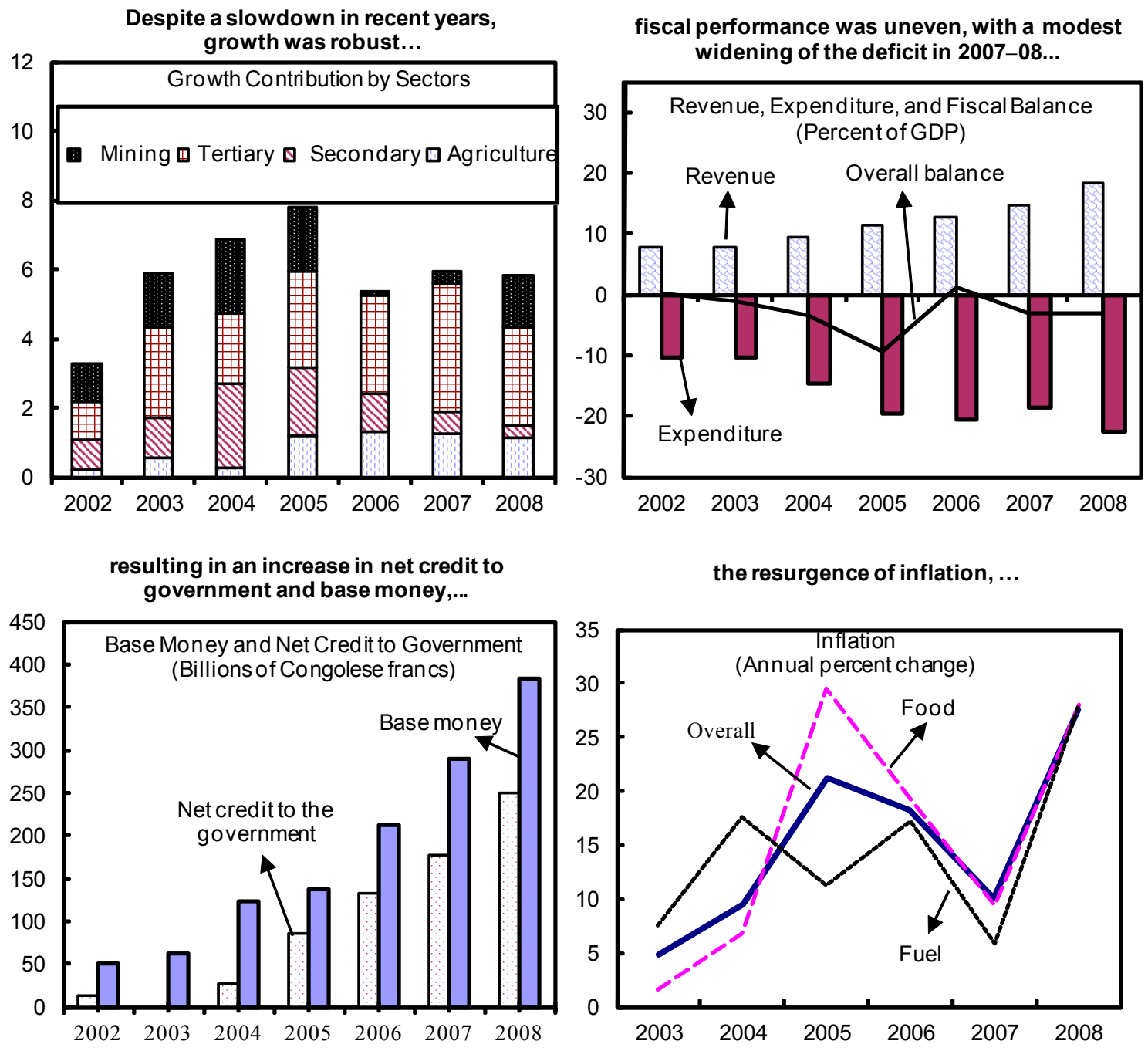

a widening of the external current account deficit, ...

... and drop in reserves.

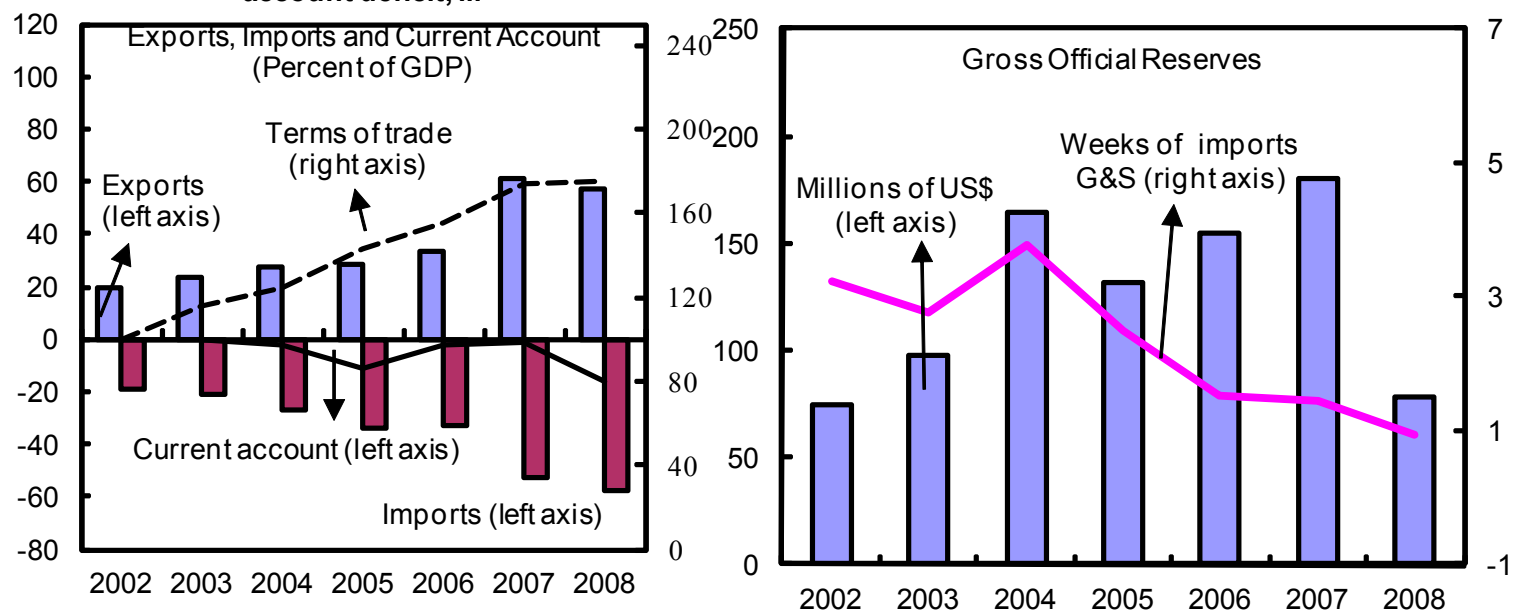

Sources: Congolese authorities; and IMF staff estimates. 


\section{To address these challenges, the authorities are requesting a new three-year PRGF arrangement to support the implementation of their PRGS. The program aims to bolster} macroeconomic stability, strengthen PFM, reinforce central bank independence, and improve the business climate. It is underpinned by an updated PRGS (2009-10) that bridges the period the authorities need to finalize their second generation PRGS (2011-16) by June 2010.

\footnotetext{
Box 1. Democratic Republic of the Congo: The Sino-Congolese Cooperation Agreement (SCCA)

In April 2008, the DRC signed a cooperation agreement with a consortium of Chinese enterprises involving a US $\$ 3.2$ billion mining project and a set of US\$6 billion public infrastructure projects to be implemented in two phases. The Agreement was amended in October 2009 to exclude the second phase public infrastructure projects, leaving just a single phase totaling US\$3 billion to be implemented over the period 2009-14. The amended agreement also limited the government guarantee to the financing of the infrastructure projects.
}

The Agreement calls for the creation of a joint venture (SICOMINES) between a Congolese parastatal mining enterprise (GECAMINES) and the consortium of Chinese enterprises. Paid-in capital of US $\$ 100$ million gave GECAMINES a 32 percent equity share in the joint venture. SICOMINE will invest US $\$ 3.2$ billion in the mining project financed by a US\$1.1 billion interest-free loan and a US\$2.1 billion loan with a fixed interest rate of 6.1 percent. The US\$3 billion investment in public infrastructure is to be financed through a series of loans at a fixed interest rate of 4.4 percent. All loans are denominated in US dollars.

Operating profits from the mining project will be used to repay the mining and public infrastructure financing. The mine is expected to generate operating profits beginning in 2013. Initially, the net profits of SICOMINES will repay principal and capitalized interest on four priority public infrastructure projects (US\$375 million). After this financing is fully repaid, 85 percent of the net operating profits will be used to repay the principal and capitalized interest on the mining loan. After the mining financing has been fully repaid, 85 percent of SICOMINES' net operating profits will be used to repay the principal and capitalized interest on the remaining public infrastructure loans and the balance will be distributed as a dividend to the SICOMINES shareholders.

The grant element of the infrastructure financing is estimated at 42-46 percent, depending whether the US $\$ 250$ million signing bonus is taken into account. This is based on the following assumptions:

(i) the mining project does not generate any net income and the government guarantee is invoked after 25 years as provided for under the amended Agreement; (ii) accrued interest is compounded; and (iii) a fixed interest rate of 4.4 percent applies. 


\section{RECENT ECONOMIC DEVELOPMENTS}

9. Macroeconomic policies were satisfactory during the first half of $\mathbf{2 0 0 8}$. The fiscal balance (cash basis) recorded modest surpluses through end-June. ${ }^{2}$ Domestic revenue performance was robust and expenditures were kept in line with available resources. This allowed the central bank to contain the growth of monetary aggregates while keeping its policy rate unchanged at 28 percent.

\section{The escalation of conflict weakened the fiscal position during the second half of}

2008. An acceleration of security-related spending together with weak PFM shifted the underlying fiscal balance to a deficit in the last quarter and limited the surplus for the year as a whole to about 1 percent of GDP - about 1 percentage point below target (Figure 3). Under these circumstances, net credit to government (NCG) by the Central Bank of the Congo (BCC) was 2 percentage points of GDP above target at year-end. This boosted base money and eased liquidity conditions.

\section{Macroeconomic policies were mixed through September 2009.}

- During the first half of the year, underlying fiscal surpluses (on a cash basis) averaged 0.1 percent of GDP, as the government slowed spending. During the third quarter, modest improvements in domestic revenue (excluding the SCCA signing bonus) combined with the government's success in containing expenditures kept the fiscal position in line with projections.

- On monetary policy, the $\mathrm{BCC}$ raised its indicative interest rate in two steps from 40 percent to 65 percent in January and stepped up sales of central bank bills. However, increased lending by the BCC to distressed banks and the failure to mop up liquidity injections by government led to periodic accelerations of base money growth during the third quarter. In response, in October the $\mathrm{BCC}$ raised its indicative interest rate further to 70 percent, increased the reserve requirement ratio from 5 to 7 percent, and stepped up sales of central bank bills.

\footnotetext{
${ }^{2}$ The underlying fiscal balance is defined as revenue minus expenditure excluding interest on foreign debt, foreignfinanced investment, and exceptional outlays.
} 
Figure 3. Democratic Republic of the Congo: Recent Economic Developments
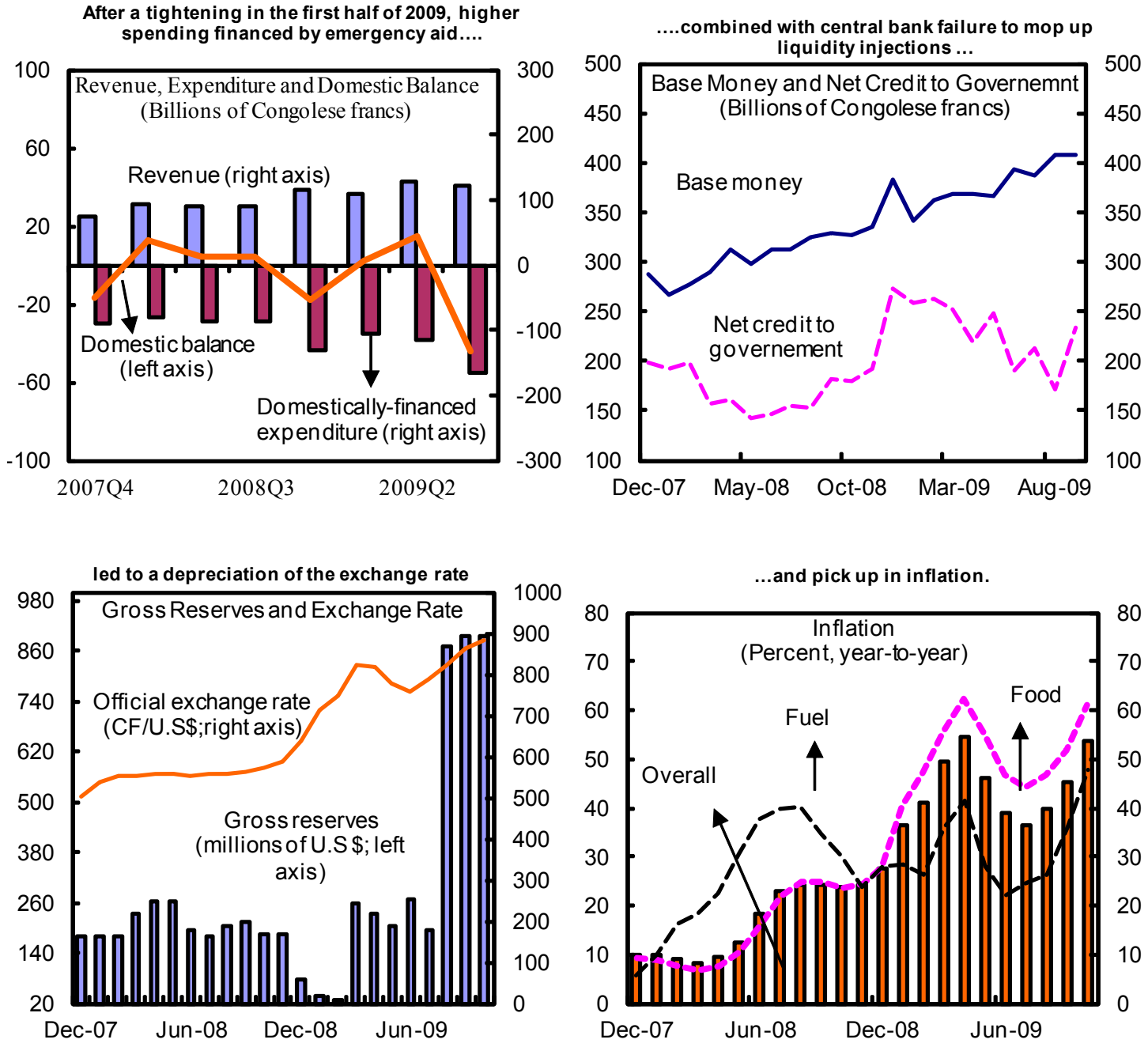

In response, the central bank tightened monetary
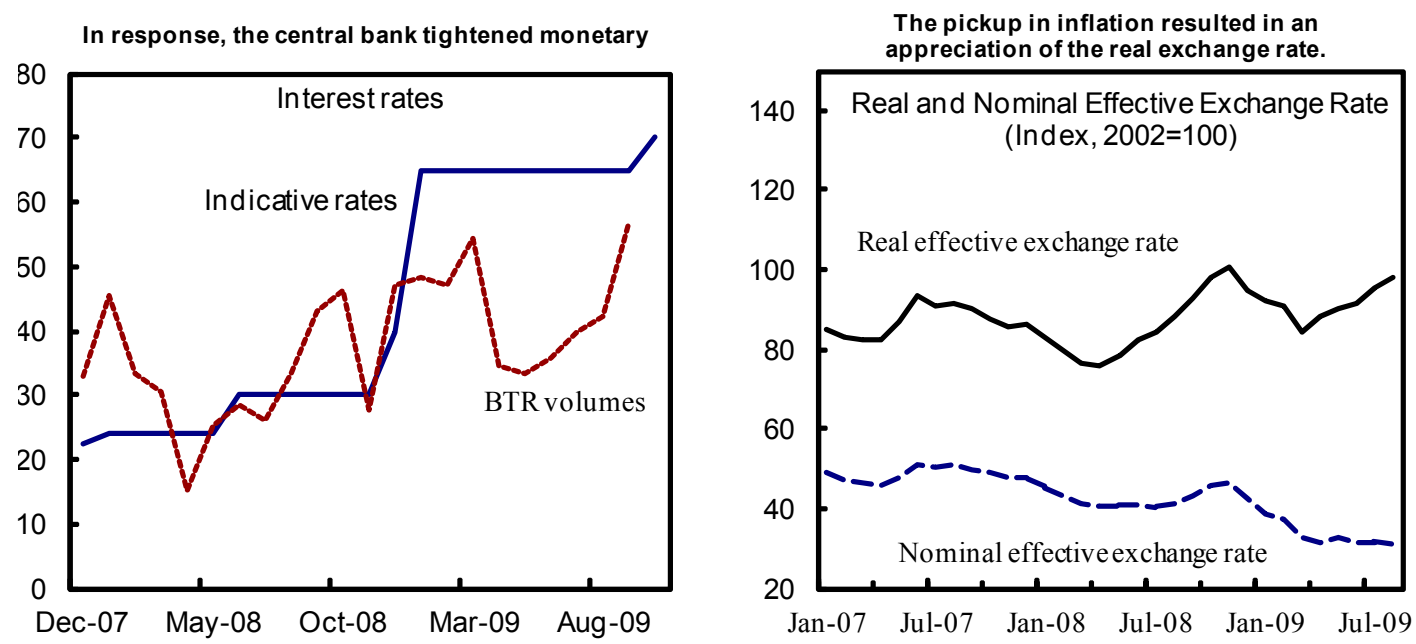

Sources: Congolese authorities; and IMF staff estimates. 


\section{Against this background, macroeconomic outcomes were mixed.}

- Economic activity. In 2008, real GDP growth was about 6 percent instead of the projected 10 percent reflecting weaker exports in the face of the global financial crisis and supply bottlenecks (especially shortages in cement and electricity) that constrained construction and manufacturing activities (Table 1). It is projected to be 2.7 percent in 2009 owing to the effects of the global financial crisis.

- Prices. There had been setbacks in containing inflation. After easing during the second half of 2008 thanks to the retrenchment of world fuel and food prices, inflation picked up in late 2008 and early 2009 owing mainly to government borrowing from the BCC. After a brief decline in May-August, inflation started to rise and reached 53 percent (year-onyear) at end-October in response to the periodic strong increases in base money and the depreciation of the Congolese franc. Under these circumstances, dollarization increased.

- Current account and exchange rate. In 2008, the external current account deficit (including transfers) widened by some 14 $\frac{1}{2}$ percentage points of GDP to about 16 percent of GDP owing to buoyant imports and the drop of export prices in second half of the year on account of the global financial crisis. The Congolese franc depreciated by 35 percent against the U.S. dollar between December 2008 and September 2009. The real exchange rate remained broadly stable over the past several years.

- Gross official reserves. Reserves declined from US\$180 million at end-2007 to about US\$78 million (one week of nonaid imports) by end-2008 and further to a historically low level of US\$30 million in February 2009. They reached US\$894 million at end-September (10 weeks of nonaid import cover) following the disbursement of emergency assistance from the Fund and other development partners, the arrival of the first tranche of the SCCA signing bonus, and the general and special SDR allocations (SDR 424.5 million).

13. Banking system soundness and vulnerability. The health of the financial sector remains fragile (Text Table 1). At end September 2009, the capital-risk-weighted adequacy ratio averaged about 15 percent (compared with the required 10 percent), up from about 11 percent at the end of 2008. A large number of banks also have been unable to observe the required liquidity and net foreign exchange position ratios. Nonperforming loans increased significantly at the end of September 2009, reflecting in part recent improvements in the accuracy of the reporting system. The contagion effects of the global financial crisis have thus far had a limited direct impact on the banking system. Nevertheless, three local banks have shown signs of distress and urgently need to be recapitalized. The BCC is implementing a comprehensive strategy to address the issue, with technical assistance from the Fund and the World Bank. Specifically, it is strengthening on-site and off-site banking supervision and tightening and enforcing prudential regulations. It has also taken initial steps regarding the restructuring of one large bank. 


\begin{tabular}{|c|c|c|c|c|c|c|c|}
\hline \multicolumn{8}{|c|}{ Text Table 1. Democratic Republic of the Congo: Financial Soundness Indicators, 2003- September 2009} \\
\hline & 2003 & 2004 & 2005 & 2006 & 2007 & 2008 & $\begin{array}{r}\text { Sep-09 } \\
\text { Prel. }\end{array}$ \\
\hline \multicolumn{8}{|l|}{ Capital adequacy } \\
\hline Regulatory capital to risk-weighted assets & -3.40 & 6.83 & 7.66 & 10.52 & 12.84 & 10.85 & 15.00 \\
\hline Regulatory Tier 1 capital to risk-weighted assets & -4.06 & 6.56 & 7.12 & 10.30 & 11.46 & 9.18 & $\cdots$ \\
\hline \multicolumn{8}{|l|}{ Asset Quality } \\
\hline Non-performing loans to total gross loans & 1.54 & 2.03 & 6.75 & 3.00 & 4.46 & 2.77 & 10.60 \\
\hline Non-performing loans net of provisions to capital & 0.06 & 4.06 & 9.28 & 6.53 & 11.72 & 10.53 & 16.88 \\
\hline \multicolumn{8}{|l|}{ Earnings and profitability } \\
\hline Return on assets & 0.55 & -1.12 & 1.66 & 2.65 & 2.52 & 2.10 & 0.60 \\
\hline Return on equity & 0.59 & -18.51 & 25.28 & 51.61 & 40.83 & 37.62 & 5.60 \\
\hline Interest margin to gross income & 18.92 & 20.91 & 32.68 & 39.77 & 87.89 & 91.89 & $\ldots$ \\
\hline Non-interest expenses to gross income & 77.36 & 86.41 & 58.02 & 44.45 & 79.28 & 79.83 & $\ldots$ \\
\hline \multicolumn{8}{|l|}{ Liquidity } \\
\hline Liquid assets to total assets (liquid asset ratio) & 47.03 & 77.19 & 88.46 & 82.99 & 79.80 & 74.77 & 75.00 \\
\hline Liquid assets to short-term liabilities & 53.64 & 81.42 & 94.96 & 92.88 & 62.85 & 103.97 & 78.00 \\
\hline \multicolumn{8}{|l|}{ Sensitivity to market risk } \\
\hline Net open in foreign exchange position to capital & 29.97 & 52.53 & 12.92 & 50.43 & 41.75 & -11.46 & $\ldots$ \\
\hline Foreign currency-denominated liabilities to total liabilities & 69.23 & 80.23 & 73.61 & 76.37 & 76.19 & 81.31 & 91.90 \\
\hline
\end{tabular}

14. Performance under the program to date was satisfactory. All prior actions and indicative targets through end-September were observed and structural reforms remained on track (MEFP, Tables 1 and 2).

\section{MEDIUM-TERM OUTLOOK}

\section{Although short-term economic conditions remain weak, the medium-term outlook is} positive. The deterioration in the country's terms of trade and the decline in FDI on account of the global financial crisis are taking their toll on economic growth in 2009, particularly in the mining sector. Growth should pick up in 2010 and average 6.5 percent in 2010-12, supported by higher public and private investment and a recovery in the mining sector. However, it will remain below the 8 percent projected during the previous Article IV consultation (IMF Country Report No. 07/327). Inflation should decline to 48.7 percent by end-2009 and to single digits by 2012 , supported by prudent fiscal and monetary policies. Given an expected gradual recovery in exports and large increases in investment-related imports, the current account deficit is expected to widen over the medium term, but will be financed in large part by capital and financial flows tied to infrastructure and mining projects. Gross reserves will remain to the equivalent of about 10 weeks of imports in 2012, in large part supported by the government's decision to save the SDR allocations and maintain them in SDRs. 


\section{Article IV Consultation and Policy Discussions}

16. Drawing on the recommendations from the 2007 Article IV consultation (Box 2), the 2009 Article IV consultation discussions centered on three main themes: (i) fostering sustained and high economic growth; (ii) enhancing external sustainability; and (iii) strengthening institutional capacity for economic management.

\begin{tabular}{|c|c|}
\hline $\begin{array}{l}\text { Improve revenue collection, including through a } \\
\text { comprehensive reform of collection agencies. }\end{array}$ & $\begin{array}{l}\text { While progress was made in improving revenue } \\
\text { collection, there have been delays in reforming the } \\
\text { collection agencies, increasing mining sector taxation, } \\
\text { and simplifying taxes. }\end{array}$ \\
\hline Improve PFM and prioritization of spending. & $\begin{array}{l}\text { There was progress in addressing the urgent spending } \\
\text { procedures, but comprehensive PFM reform remains } \\
\text { at infancy stage and the lingering conflict undermines } \\
\text { spending prioritization. }\end{array}$ \\
\hline Reform the financial sector. & $\begin{array}{l}\text { Some progress has been made in strengthening } \\
\text { banking supervision and reinforcing compliance with } \\
\text { prudential regulations, but efforts to restructure weak } \\
\text { banks has been slow. }\end{array}$ \\
\hline $\begin{array}{l}\text { Address fiscal dominance and enhance central bank } \\
\text { independence. }\end{array}$ & $\begin{array}{l}\text { Reducing fiscal dominance has been hampered by } \\
\text { periods of increased government borrowing from the } \\
\text { central bank partly related to the lingering conflict in } \\
\text { the eastern provinces; restructuring of the central bank } \\
\text { is proceeding. }\end{array}$ \\
\hline $\begin{array}{l}\text { Implement structural reforms to enhance the } \\
\text { economy's supply response. }\end{array}$ & $\begin{array}{l}\text { Initial steps were taken to prepare key public } \\
\text { enterprises for privatization, but reforms to streamline } \\
\text { the regulatory environment and address governance } \\
\text { problems have been slow. }\end{array}$ \\
\hline
\end{tabular}

\section{A. Fostering Sustained and High Economic Growth}

\section{Sustained and high economic growth is critical to increasing per capita income and} reducing poverty. The authorities agreed with staff that this requires bolstering macroeconomic stability, creating fiscal space for the provision of critical public goods, and advancing structural reforms to foster private sector development.

18. Moving toward macroeconomic stability. Given the country's history of hyperinflation and uneven implementation of policies, staff believe that the fiscal policy rule of zero net bank credit to the government is critical to taming inflation and addressing fiscal dominance over monetary policy. The central bank would need to remain vigilant and mop up any excess liquidity, particularly those related to donor-financed public spending. The authorities agreed on the need to avoid recourse to central bank borrowing as this would reduce the burden on monetary policy in an environment where its effectiveness is already limited by the high degree of dollarization and underdeveloped financial markets. The authorities and staff also concurred that the reorganizing, restructuring, and recapitalizing the central bank is critical to enhancing its independence and capacity to conduct monetary and exchange rate policy. 
19. Increasing fiscal space for priority spending. To create such space, the authorities need to mobilize domestic revenues and strengthen PFM. Staff noted the low levels of tax collection to date, particularly in the mineral sector, and the importance of increasing domestic revenues to address the country's large reconstruction needs. The authorities agreed that bold reforms are needed to modernize and consolidate tax and customs management, streamline exemptions, and simplify the tax system, including introducing a VAT. They maintained, however, that this would need to be addressed over the medium term given capacity constraints. However, they have already begun taking steps to strengthen mining sector tax collection, including by reviewing exemptions and establishing a unit within the tax administration dedicated to this sector.

20. PFM. Poor budget planning, circumvention of budgetary procedures, and ineffective budget controls have weakened fiscal discipline and accountability. This, together with high levels of nondiscretionary spending, impedes progress in achieving the country's poverty reduction objectives. Staff stressed the importance of realistic budgets, following proper budgetary procedures, strengthening controls, and improving priority expenditure tracking. Strengthening institutional capacity in PFM at the provincial level, with technical assistance from development partners, will also be important given the ongoing decentralization. The authorities concurred with the staff's assessment and noted that progress on these fronts would also help allay concerns over governance. They stressed that the speed of PFM reforms should take into account the country's post-conflict situation.

21. Improving the business climate. Staff urged the authorities to press ahead with reforms to reduce the cost of doing business and to diversify the economy. The authorities indicated that they are placing emphasis on restructuring public enterprises and privatizing the management of key public services (i.e., transport and utilities) as a first step toward privatization. They plan to submit to parliament a law that will allow the DRC to participate in the Organization for the Harmonization of Business Law in Africa (OHADA), which will help simplify procedures for business registration and strengthen legal protection of foreign investment. The authorities also noted that they established and staffed the office responsible for the implementation of the 2004 anti-money laundering and combating financing of terrorism legislation. Staff welcomed these efforts, but encouraged the authorities to advance reforms in the areas of banking supervision, credit information management, and the judiciary.

\section{B. Enhancing External Sustainability}

\section{A flexible exchange rate regime is appropriate given the country's low level of} foreign reserves and vulnerability to exogenous shocks (Box 3). Staff noted that the exchange rate has broadly tracked economic fundamentals and appears appropriate assuming that donor flows continue and the DRC benefits from debt relief under the enhanced HIPC Initiative and Multilateral Debt Relief Initiative (MDRI). The authorities concurred, noting that the central bank intervenes in the foreign exchange market to achieve its reserve target and to smooth sharp fluctuations in the rate. Staff stressed that this could best be addressed by advancing reforms to reduce the cost of doing business. 


\section{Box 3. The Democratic Republic of the Congo: An Assessment of the Exchange Rate Policy and External} Competitiveness

The exchange rate of the Congo franc has been freely floating since the liberalization of the foreign exchange market and the unification of the exchange rate in 2001. Since then, BCC intervention in the foreign exchange market has been limited. Thus, the exchange rate appears to have been responsive to changes in macroeconomic policies and exogenous shocks. The nominal effective exchange rate of the Congo franc (NEER) depreciated by 10 percent a year on average over 2002-08 while the real effective exchange rate (REER) appreciated by 2 percent on average over the same period. Although assessing the equilibrium exchange rate is a complicated exercise, particularly in a post-conflict country with poor data and significant structural breaks, two approaches were used to assess the consistency of the real exchange rate with its equilibrium level.

1. An estimation of the long-run trend of the real exchange rate by using standard smoothing filters-Fiveyear moving average of the REER and the Hodrik Prescott (HP) filter - to isolate the permanent component from the transient and short term shocks. The smoothed variable is then interpreted as the equilibrium real exchange rate. In 2002-08, the actual REER was below its equilibrium level most of the period, but appears to have converged to its equilibrium level at the end of the period.

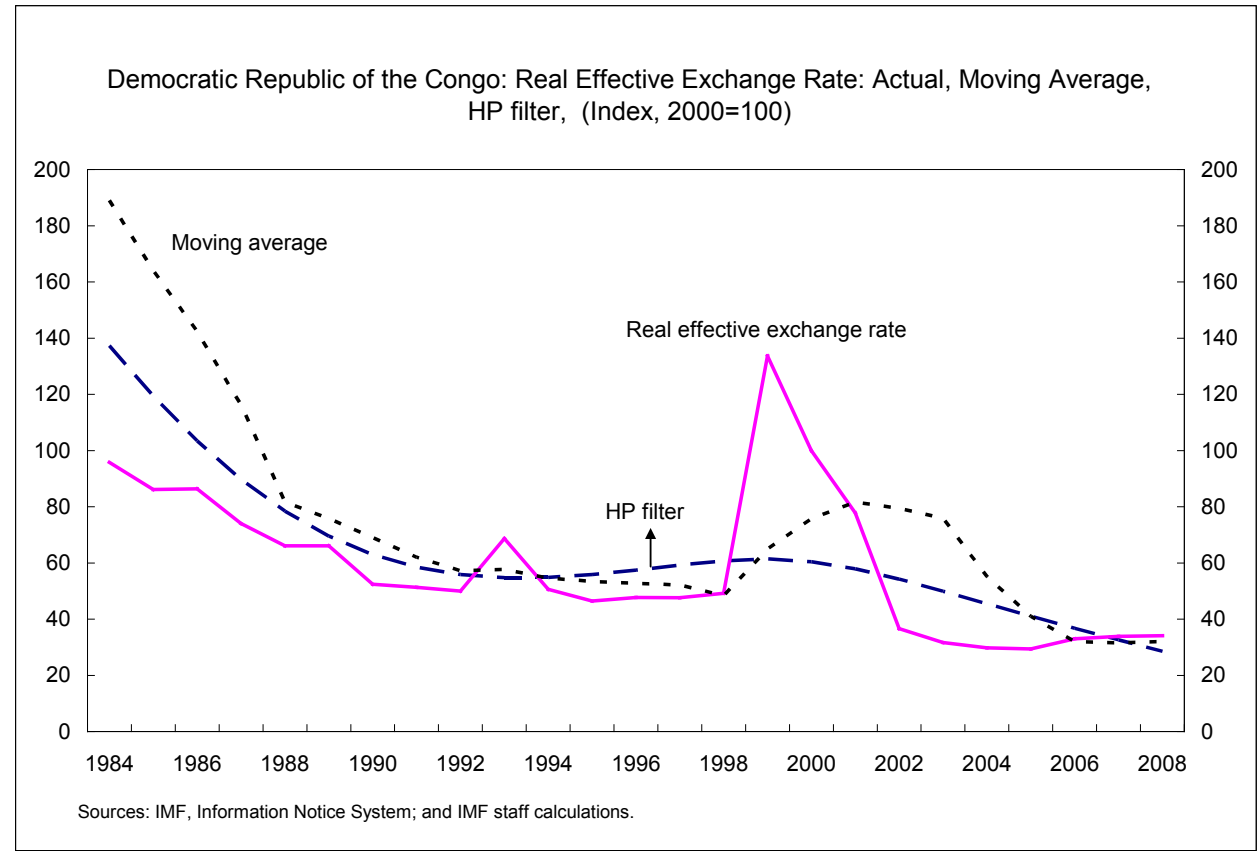

2. An application of the macro-balance approach developed by the Consultative Group on Exchange Rate Issues. It consists of three steps. First, estimating an underlying current account (UCA) based on the medium term framework, excluding temporary factors. Second, computing a current account norm (CAN) based on the fundamentals. Third, deriving the adjustment in the REER necessary to close the gap between UCA and CAN, assuming import and export elasticities. Using different approaches, the UCA is estimated at a deficit of around 12 percent of GDP. The CAN is derived from variables and estimated coefficients on a panel of developing countries (see Isard and others (IMF Occasional Paper, 2001). Essentially driven by low level of net foreign assets and foreign aid, the CAN is estimated at about -8.4 percent. Using standard export and import elasticities, the analysis suggests the exchange rate would need to depreciate by about 9 percent to close the gap between the UCA and the CAN.

The above results do not suggest significant over or under valuation of the exchange rate. However, the inability by the DRC to fully service its debt (and thus accumulate arrears on its external debt) suggests that macroeconomic policies and structural reforms were insufficient to generate the growth and resources needed to fully meet debt service obligations. Nevertheless, the exchange rate level would be appropriate if foreign aid flows continue and the DRC benefits from debt relief from the enhanced HIPC initiative and the MDRI. 
23. The DRC's debt situation is unsustainable absent substantial debt relief. Debt burden indicators markedly exceed their policy-based thresholds. ${ }^{3}$ In the absence of debt relief from the enhanced HIPC Initiative and the MDRI, external debt burden indicators are projected to remain above policy-dependent thresholds over prolonged periods. The DRC will continue to be vulnerable to adverse exogenous shocks even after access to debt relief under the enhanced HIPC Initiative and MDRI reduces the country's debt burden indicators below the thresholds. It is expected to reach the HIPC Completion point at earliest during the second quarter of 2010 , assuming completion of the first review of the PRGF arrangement, indications that program implementation through end-March 2010 remains satisfactory, one-year satisfactory implementation of their PRGS, and observance of the HIPC triggers (Table 7). The authorities committed to adhere to a debt strategy that minimizes the risks of debt stress by relying strictly on grants or highly concessional loans. In this context, they have agreed with their partners to remove from the SCCA the second phase infrastructure projects and the government guarantee on the mining project.

\section{Strengthening Institutional Capacity for Economic Management}

\section{Fragmented institutions and political considerations have complicated policy}

management. The bifurcation of the ministries in charge of finance and budget impedes efficiency and coordination of fiscal policy. This, along with weak monetary policy coordination and fragmentation of responsibilities across other ministries, complicates policy implementation and reform. These weaknesses would likely be exacerbated by the constitutionally mandated fiscal decentralization policy given weak PFM at the provincial level. Staff encouraged the authorities to merge the ministries in charge of finance and budget, improve fiscal and monetary policy coordination, and strengthen the institutional capacities of provinces. The authorities agreed, but stressed that this should be seen in the context of the country's nascent institutions and broad-based government coalition that has played a critical role in national reconciliation.

\section{Improvement in economic statistics is critical for program implementation and} monitoring. Weaknesses in data preparation, timeliness, and quality persist on account of inadequate human and material resources and weak coordination. Staff urged the authorities to adopt a new statistics law, finalize their national strategy for statistics development, and complete the new national accounts. The authorities indicated that they would do so, but stressed that rebuilding capacity in post-conflict setting would require substantial donor assistance.

\section{The Program FOR 2009-12}

26. The authorities are requesting a three-year PRGF arrangement and disbursement of additional interim assistance under the enhanced HIPC Initiative to support their economic program over the period July 2009 to June 2012. The program's objectives are: (i) average real GDP growth of 5.5 percent; (ii) end-period inflation rate of 9 percent by 2012;

\footnotetext{
${ }^{3}$ See the joint Bank/Fund Debt Sustainability Analysis.
} 
(iii) gross reserves equivalent to 10 weeks of nonaid imports by 2012; and (iv) the external current account deficit (including grants) limited to an average of 25 percent of GDP. The program supports the implementation of the authorities' PRGS (Box 4) and underpinning policies and reforms, which are presented in the attached Letter of Intent (LOI), Memorandum of Economic and Financial Polices (MEFP), and Technical Memorandum of Understanding (TMU). The program focuses on enhancing macroeconomic stability, mobilizing domestic revenues, strengthening PFM, reforming the central bank, and improving the business climate.

Box 4. The Democratic Republic of the Congo: Poverty Reduction and Growth Strategy (PRGS)

The authorities completed their first full PRGS paper covering the period 2006-08 through a participatory process in July 2006. It was endorsed by the democratically elected government in March 2007. The strategy is based on five pillars:

- $\quad$ Promotion of peace and good governance;

- Consolidation of macroeconomic stability and promotion of economic growth;

- Improvement in access to social services;

- $\quad$ Fight against HIV/AIDS; and

- $\quad$ Promotion of a dynamic community

In their PRSP Progress Report dated July 2008, the authorities acknowledged that the strategy was overly ambitious and its implementation hindered by the conflict in the eastern provinces, capacity constraints, and budgetary constraints. As a result, there was little progress in achieving the PRGS objectives and key social indicators did not improve significantly, with the exception of primary education enrollment and maternal mortality rates. Delays in rebuilding the democratic institutions also affected the government's ability monitor key reforms.

With a view to ensuring the achievement of the PRGS objectives despite the problems experienced to date, the authorities decided to extend their strategy for an additional 18 months through the end of 2010 . They have adopted an updated Priority Action Plan (PAP2) for the period 2009-10 that seeks to correct the weaknesses identified in the progress report by prioritizing the key policies and actions to be implemented. Aligning the budget with these priorities will be a key element of the government's strategy going forward. Given that the poverty dynamics in the DRC remain unchanged, staff believes that the current PRGS remains as the appropriate basis for underpinning the PRGF in 2009 and 2010.

In parallel, the authorities are preparing their second generation PRGS, which will cover the period 2011-15. This document will be developed in a participatory manner, including at the provincial level, and is expected to be completed and approved by December 2010.

\section{A. Macroeconomic Policies}

\section{Macroeconomic policies aim at reducing inflation and supporting strong and} sustained economic growth. Fiscal policy will avoid recourse to central bank financing and lay the foundation for long-term sustainability. Absent fiscal dominance, monetary policy will focus on reducing inflation to single digits in the context of a flexible exchange rate regime.

\section{Fiscal policy}

\section{The budget deficits will be capped by the availability of concessional foreign}

assistance. In this context, the underlying fiscal balance (cash basis) is targeted to shift from a deficit of 2.1 percent of GDP in 2009 to 0.4 percent in 2012 (Table 2). This fiscal adjustment is consistent with the government's objectives of zero net borrowing from the banking sector and, 
thus, reducing fiscal dominance over monetary policy. ${ }^{4}$ Under the assumption that the DRC receives substantial debt relief under the enhanced HIPC Initiative and MDRI, the program's medium-term fiscal adjustment is consistent with fiscal sustainability over the long term. ${ }^{5}$ Domestic revenue (excluding the signing bonus from the SCCA) is projected to increase from 15.7 percent of GDP to about 20 percent over the same period. The projections are reasonable given DRC's post-conflict situation whereby economic recovery, expansion of central government's authority over the eastern provinces, the teething of tax and customs administration reforms, and the introduction of a VAT are expected to improve revenue collection. ${ }^{6}$ This, along with scaled up development assistance, should allow higher domestic spending on priority programs. The government is committed to reduce nonpriority spending (including investment and transfers) in the event that domestic revenue or external budget support are lower than envisaged. Further, the fiscal program provides for a budget reserve for unanticipated events, such as natural disasters and security-related spending. The staff will continue to work closely with the authorities to improve reporting on budget execution, including information on social and other priority spending.

29. The resolution of the conflict in the eastern provinces and the improved relationship between the DRC and its neighbors will reduce security-related spending. In recent years, security-related spending constrained other priority spending and complicated budget planning. Over the medium term, improved security conditions will help enhance expenditure allocation, reduce recourse to urgent spending procedures, and make budget planning more predictable This should facilitate the implementation of the second generation PRGS.

\section{Monetary and exchange rate policies}

30. The objective of monetary policy is to achieve single-digit inflation by 2012. Progress on this front is critical to fostering confidence in the local currency. Base money will remain as the nominal anchor. Pending the development of a treasury bill market, the BCC will primarily use central bank bills to mop up excess liquidity (MEFP q21). As necessary, it will auction foreign exchange to sterilize liquidity arising from donor-financed government spending. The BCC will improve its liquidity forecasting in close collaboration with the Treasury.

31. The BCC will rebuild its gross official foreign reserves. The program objective is to maintain gross official reserves at the equivalent of 10 weeks of import coverage by 2012 . This level corresponds to slightly below one standard deviation of export levels and should therefore be adequate, particularly given the country's free-floating exchange rate regime. The BCC will intervene in the foreign exchange market only to smooth large fluctuations in the rate and to achieve the foreign reserve target (MEFP $\mid 21) .{ }^{7}$ To support reserve build up while fostering financial market development, the authorities are ceasing making payments in foreign exchange to domestic suppliers and requiring tax payments to be made in local currency.

\footnotetext{
${ }^{4}$ The zero net credit to government target for the year as whole is consistent with net borrowing within the year for treasury management purposes. The ceiling will limit undue exposure to domestic debt and the crowding out of private sector credit.

${ }^{5}$ The program also includes a zero ceiling on nonconcessional external borrowing.

${ }^{6}$ In 2002-08, the revenue-GDP ratio increased on average by some 2 percentage points notwithstanding the effects of the lingering conflict in the eastern provinces and slow implementation of reforms.

${ }^{7}$ The central bank may also intervene to avoid market disruptions due to the small size of the market.
} 


\section{B. Structural Reforms}

32. The authorities' structural reform agenda will focus on domestic revenue mobilization, PFM, central bank reform, and private sector development. The proposed reforms are drawn from those outlined in the PRGS. Given the complementarity of reforms in achieving the authorities' objectives, the PRGF-supported program will be coordinated closely with other development partners (Text Table 2).

33. Domestic revenue mobilization. The focus is on expanding the tax base by streamlining tax exemptions in the investment, mining, and forestry codes with a view to eliminating them over time. Strengthening the Large Taxpayers Unit at the income tax department will improve tax collection from mining companies (MEFP 926 ), while the introduction of a VAT in 2012 will allow for the removal of various discretionary taxes (MEFP q23). To prepare for this, the authorities plan to establish tax collection centers for medium-sized enterprises across the country. Customs administration reform aims at reducing fraud, shortening clearance delays, and improving valuation (MEFP 924 ). The customs administration will be transformed into a department of the Ministry of Finance with its own budget. Key priorities will include improving the monitoring of mining exports, expanding one-stop customs windows at border posts, modernizing information management, enforcing pre-inspection regulations and enhancing antifraud activities, and implementing a single taxpayer identification number.

34. PFM. The focus is on strengthening budget preparation, execution, and accountability (MEFP -27). The authorities will adopt an organic public finance law aimed at rationalizing and harmonizing the budget process. Budget planning and control will be strengthened by developing a medium-term expenditure framework in cooperation with line ministries and by requiring all government transactions to adhere to proper budget procedures. Other key reforms include the introduction of a double entry accounting system at the treasury, enhancement of ex ante and ex post budget controls, and preparation and implementation of an action plan to strengthen PFM at the provincial level.

35. Central Bank reform. The focus is on restoring the BCC's independence (MEFP 930 ) and strengthening its supervisory capacity (MEFP \31) with the technical assistance from the IMF's Monetary and Capital Markets (MCM) department. Given the BCC's limited income base-low seignorage (due to dollarization), and interest earnings on foreign reserves (due to low level of interest-bearing reserves), restructuring and reorganization are critical to make the bank more efficient. This in turn will facilitate its recapitalization by the government. Strengthening banking supervision will involve improving the legal and regulatory framework, and reinforcing banking supervision capacity, including human and material resource requirements.

36. Private sector development. Key elements include reforming public enterprises (MEFP \37), streamlining government regulations and protecting foreign investment (MEFP $\llbracket 33$ ), and improving transparency in natural resource management by adhering to the EITI (MEFP q36). 
Text Table 2: The Democratic Republic of the Congo: Structural Reform and Burden Sharing

\begin{tabular}{lll}
\hline & IMF & Other Agencies \\
$\begin{array}{l}\text { Fiscal Structural Reforms } \\
\text { Tax and customs administration }\end{array}$ & IMF & World Bank \\
$\begin{array}{l}\text { Public financial management } \\
\text { Decentralization }\end{array}$ & IMF & World Bank \\
Debt management & IMF & World Bank \\
& IMF & World Bank/UNCTAD \\
Monetary and Financial Sector Reforms & & \\
$\quad$ Central Bank reform & IMF & \\
$\begin{array}{l}\text { Monetary operations } \\
\text { Foreign exchange market reforms }\end{array}$ & IMF & Belgium \\
$\begin{array}{l}\text { Payment system } \\
\text { Banking supervision }\end{array}$ & IMF & \\
$\begin{array}{l}\text { Restructuring of financial institutions } \\
\text { Microfinance }\end{array}$ & IMF & World Bank/USAID \\
$\begin{array}{l}\text { Other Reforms } \\
\text { Civil service }\end{array}$ & World Bank \\
$\begin{array}{l}\text { Public enterprises } \\
\text { Regulatory environment for business }\end{array}$ & & World Bank \\
Judicial & & \\
Extractive industries & & World Bank \\
Governance & & World Bank \\
Trade & & World Bank \\
\hline
\end{tabular}

VI. The 2009 AND 2010 Program

37. The program objectives for $\mathbf{2 0 0 9}$ are: (i) real GDP growth of 2.7 percent; (ii) an end-ofperiod inflation rate of 48.7 percent; and (iii) gross official reserves equivalent to 10 weeks of nonaid imports.

\section{Macroeconomic policies}

38. The fiscal program targets a reduction in government borrowing from the banking sector. The underlying fiscal deficit (cash basis) is therefore targeted at 2.1 percent of GDP, a deterioration of about 3 percentage points of GDP relative to 2008. This will allow a reduction in net credit to government of about 1.2 percent of GDP, reflecting the savings of a large portion of the signing bonus of the SCCA. To reach the deficit target, domestic revenues (excluding the signing bonus) are projected at 15.7 percent of GDP (about 3 percentage points less than in 2008), reflecting mainly the impact of lower petroleum prices and weak economic activity. Further, domestically financed spending (outside exceptional outlays) is targeted at 16.3 percent of GDP.

39. The fiscal objectives for the remainder of 2009 appear to be achievable. Domestic revenue is expected to be line with program objectives for the year as a whole. On the expenditure side, the authorities have taken corrective measures to address a large build up of expenditure commitments in October that could have threatened the fiscal targets. Specifically, they cancelled all commitments for which goods and services have not yet been delivered 
(CF 68 billion) and delayed the repayment of arrears from 2007 and 2008 pending the outcome of an ongoing audit (CF 31 billion). They also ceased all new expenditure commitments as of end-October in line with budget procedures and reallocated credits for discretionary spending to offset higher priority spending (i.e., goods and services and domestically financed investment). These measures should keep the underlying fiscal deficit in line with the program objective.

40. In 2010, fiscal policy will further enhance macroeconomic stability. Staff reached understandings on a 2010 fiscal framework, which is consistent with draft budget submitted by government to parliament. The underlying fiscal deficit (cash basis) is projected to decline to 0.5 percent of GDP in 2010. Higher domestically financed expenditure outside exceptional spending (18 percent of GDP or about 2 percentage points more than in 2009) will be accommodated by an increase in domestic revenues. Foreign-financed capital expenditure, which includes the infrastructures projects implemented under the SCCA, is projected to reach 14 percent of GDP. The resulting overall fiscal deficit on a cash basis (12.7 percent of GDP) will be entirely financed through external budget and project support, with no recourse to domestic financing. In this regard, the authorities have committed to reduce spending - mainly in the areas of goods and services and domestically financed investment in non-priority programsin the event that domestic revenue or external budget support is lower than programmed.

41. Monetary policy for the remainder of $\mathbf{2 0 0 9}$ and in $\mathbf{2 0 1 0}$ aims to reduce further endyear inflation to 48.7 percent and 15 percent, respectively. The $\mathrm{BCC}$ intends to maintain a tight monetary policy stance to achieve its inflations objectives. In this context, base money growth is targeted at 19.6 percent and 22 percent in 2009 and 2010, respectively, in line with nominal GDP growth and the inflation objectives (Table 3). In addition to the use of central bank bills to achieve the monetary targets, the BCC may auction foreign exchange while achieving its gross reserves target. The central bank will maintain its current policy rate to support disinflation.

42. The program is fully financed. After taking into account debt relief under the enhanced HIPC Initiative and MDRI, the remaining balance of payments financing gap for July 2009-June 2010 is estimated at about US\$154 million. This is expected to be covered by the first and second disbursements under the PRGF. The financing gap for the period July-December 2010 is estimated at US\$355 million and is expected to be covered by budget support from the World Bank (US\$100 million), the European Commission (US\$66 million), and bilateral development partners (US\$189 million). In the event of a shortfall in external budget support, the disbursement of the second tranche of the SCCA signing bonus (US\$125 million), which is expected in December 2009, could be used.

\section{Structural reforms}

43. Structural reforms in $\mathbf{2 0 0 9}$ and $\mathbf{2 0 1 0}$ will focus on the areas of tax and customs administration, PFM, and the financial sector (MEFP $q 42$ ). 
44. Fiscal structural reforms aim at strengthening tax administration and PFM. The focus is to establish new tax centers for medium-sized enterprises in key provinces while strengthening the existing ones. Customs administration will also be improved by computerizing border posts and increasing the diffusion of one-stop windows. The simplification and rationalization of revenue collection will be accompanied by the assessment and elimination of nuisance taxes. On PFM, key reforms include bolstering budget planning and execution by, inter alia, adopting an organic budget law, eliminating extra budgetary spending, enhancing the PFM capacities of local governments, and improving the reliability of fiscal reporting.

45. Financial sector reforms would focus on strengthening the role and independence of the central bank. The BCC will first implement a new reorganization and restructuring plan that will help put the bank's financial situation on sustainable financial footing. The government will in turn adopt a recapitalization plan for the BCC to restore its independence. With regard to banking supervision, the focus will be on completing financial audits of the commercial banks and developing restructuring plans for those that are financially weak. The BCC is tightening prudential ratios and their monitoring, in line with recommendation by IMF technical assistance. Further, the authorities have initiated the implementation of the 2004 anti-money laundering and combating financing terrorism legislation.

\section{Access and Program Monitoring}

\section{Access under the proposed program is consistent with the country's balance of} payments needs. The proposed access of 65 percent of quota (SDR 346.45 million) takes into account the strength of the program, projected donor contributions, and the high level of external debt. The level of access is relatively modest in light of the severe debt situation and the substantial risks to the program. Fund financial support would represent 38 percent of the balance of payments need over 2010-12 (Tables 4 and 5). Disbursements will be conditioned on the completion of semiannual reviews. Access levels can be revisited and augmented if need be after the Completion point and/or in the event of a subsequent exogenous shock. The authorities are also requesting additional interim assistance under the enhanced HIPC initiative in the amount of SDR 45.66 million (20 percent of the total HIPC commitment) to help cover 51.1 percent of the principal repayments falling due to the IMF during the twelve-month period from December 2009. ${ }^{8}$

\section{The program will be monitored based on a set of quantitative semiannual} performance criteria and quarterly indicators, while structural reforms will be assessed in the context of semiannual reviews. The proposed program would begin on July 1, 2009. The quantitative performance criteria and indicators include ceilings on: (i) net credit to government by the banking sector; (ii) net domestic assets of the central bank; (iii) net foreign assets of the central bank; (iv) central bank payments on government expenditure without prior authorization

\footnotetext{
${ }^{8}$ The Fund committed SDR 228.3 million in HIPC assistance at the decision point, of which SDR 3.394 million was disbursed as interim assistance in three tranches during 2003-05, before DRC's last PRGF-supported program went off-track.
} 
by the ministers of finance and budget; (v) external arrears; and (vi) nonconcessional external debt (MEFP, Table 1). The first review is to be completed by end-June 2010 and would cover macroeconomic developments and policies for 2010 and progress in structural reforms in tax and customs administration, PFM, and the financial sector. The second review is to be completed by end-December 2010 and would cover macroeconomic developments and policies for 2011 and debt sustainability after debt relief.

48. In the context of the proposed PRGF, an update safeguards assessment of the BCC is currently in progress. The previous update assessment, concluded in January 2008, found a strengthened external audit mechanism, but significant safeguards risks remained in the control framework of the central bank, including serious concerns regarding the bank's independence. As a result, the assessment recommended specific measures for implementation in advance of a Board discussion of a new PRGF arrangement. The BCC agreed with the findings of the assessment and committed to implementing the safeguards recommendations, some of which were prior actions and others are structural benchmarks in the proposed PRGF program. Staff is monitoring BCC's implementation of the measures. The update assessment will be completed by no later than the first review.

49. The authorities have strengthened their program monitoring capacity at both the political and technical level with a view to ensuring coordination across ministries. They have created a Poverty Reduction Strategy National Steering Commission (PRS-NSC) under the authority of the Prime Minister which is responsible for supervising two commissions involved in the design and implementation of the poverty reduction and growth strategy (MEFP q46). An interministerial technical committee will serve as the main counterpart to Fund staff regarding program monitoring, reporting, and implementation.

\section{CAPACITY TO REPAY THE FUND AND RISKS}

50. The DRC's capacity to repay the Fund is satisfactory, assuming debt relief (Table 6). There are significant risks that, absent higher external balance of payments support and debt relief under the enhanced HIPC Initiative and MDRI, the DRC would be in a difficult position to meet its obligations to the Fund. Assuming such external financing and debt relief, repayment obligations to the Fund would peak at about 3.4 percent of exports in 2009-10 and average about 1 percent of exports in 2010-22. Purchases under the proposed PRGF program would not have a significant impact on the external debt outlook. They would raise the present value of external public and publicly-guaranteed debt by a maximum of 2.9 percentage points in 2012 and decline below 0.7 of a percentage point by 2019 .

51. The implementation of the program is subject to downside risks. An escalation in the conflict in the eastern provinces and/or exogenous adverse shocks such as a drop in the terms of trade could dampen economic growth, reduce revenues, worsen macroeconomic imbalances, and increase inflation and exchange rate volatility. Delayed financial sector reforms could further weaken the health of the financial sector and destabilize the economy. Weak PFM and government acquiescence to expenditure pressures (especially on public sector wages) could undermine the fiscal program and place pressures on inflation and the exchange rate. Lack of strong political will to carry forward proposed reforms could also derail the program. 


\section{StAfF APPRAisal}

52. The DRC faces critical development challenges. Although there has been good progress in macroeconomic stabilization, challenges remain to establish sustainable growth and fiscal space for poverty reduction. In this context, staff welcome the authorities' ambitious reform agenda, but implementation will require strong leadership to build a broad consensus in support of the program.

\section{Macroeconomic stabilization requires a prudent and credible fiscal policy.}

Expenditure discipline will be critical to achieving the program's fiscal objectives. The recent actions by the government to reduce expenditure commitments and delay the repayment of arrears pending their audit are encouraging. However, durable fiscal discipline will, inter alia, require the alignment of the treasury and expenditure commitment plans in order to avoid an excessive build up of commitments as in 2009. Progress on this front will help avoid a build up in domestic arrears and reduce central bank financing of the deficit. In this context, the authorities' decision to bolster the coordination of fiscal policy implementation, including through the active participation of the council of ministers, is welcome.

54. Priority spending needs to be safeguarded. Improvements in domestic revenue in the context of the expected economic recovery should help increase priority spending in line with the PRGS. A prudent wage policy is also critical for achieving the established macroeconomic objectives while avoiding the crowding out of priority spending programs.

55. Strengthening PFM is paramount to enhancing the efficiency and credibility of the budget process. This involves strengthening revenue collection, rationalizing spending procedures, minimizing recourse to urgent spending procedures, and improving priority expenditure tracking. Civil service reform and strengthened payroll management will also be essential to containing the wage bill.

56. A tight monetary policy stance is critical for reducing inflation. In this regard, the recent tightening of monetary policy by the BCC in response to a pick in inflation is welcome. This policy needs to be maintained in order to achieve inflation objective. To improve the effectiveness of monetary policy, the BCC should implement MCM TA recommendations regarding improving liquidity forecast in close collaboration with the Treasury, deepening monetary policy instruments and foreign reserve management. It should also improve the transparency of its operations in order to increase the credibility of its monetary policy. Foreign exchange market interventions should be limited to smoothing daily fluctuations and bridging private sector demand with forex generated from donor flows.

57. Structural reforms to help enhance the economy's supply response should be a high priority. The focus should be on addressing delays in mining sector and public enterprise reforms, accelerating adherence to the EITI, and reforming the judiciary. The recent establishment of the office responsible for the implementation of the 2004 anti-money laundering and combating financing terrorism is welcome. 
58. Debt sustainability hinges on debt relief. In the absence of debt relief under the HIPC Initiative and MDRI, external debt burden indicators are projected to remain above policydependent thresholds over prolonged periods. It is therefore crucial that the government implements all measures (including the HIPC triggers) required to reach the completion point under the enhanced HIPC Initiative as soon as possible. Strengthening debt management, including the avoidance of nonconcessional public debt, will also be important. The amendment to the SCCA that made it consistent with debt sustainability is welcome.

59. The staff is of the view that the Lending into Arrears Policy should be applied. This is based on the financing assurances review conducted by staff, the good faith efforts exerted by the authorities toward reaching collaborative agreements with commercial creditors, and the fact that relations with commercial creditors would not undermine the program.

60. There are downside risks to the program. An escalation in the conflict in the eastern provinces and adverse exogenous shocks could dampen economic growth, weaken domestic revenue, and worsen macroeconomic imbalances. Weak PFM and institutional capacity could also undermine the program. These risks can be lowered by the ongoing internationally supported peace efforts and increased donor financial and technical assistance.

61. The authorities' program promotes sustained high levels of growth and poverty reduction. Staff supports the authorities' request for a three-year PRGF arrangement and additional interim assistance under the enhanced HIPC Initiative. This will help close the remaining balance of payments financing gap and catalyze donor assistance to support the DRC in implementing its development agenda. Staff believe that satisfactory assurances regarding interim HIPC debt relief are in place from the African Development Fund and the International Development Agency.

62. It is proposed that the next Article IV consultation cycle with the DRC be held in accordance with the provisions on the decision on consultation cycles approved on July 15, 2002. 
Table 1. Democratic Republic of the Congo: Selected Economic and Financial Indicators, 2007-14

\begin{tabular}{|c|c|c|c|c|c|c|c|c|}
\hline & 2007 & 2008 & 2009 & 2010 & 2011 & 2012 & 2013 & 2014 \\
\hline & & Prel. & Est. & \multicolumn{5}{|c|}{ Projections } \\
\hline & \multicolumn{8}{|c|}{ (Annual percentage change; unless otherwise indicated) } \\
\hline \multicolumn{9}{|l|}{ GDP and prices } \\
\hline Real GDP & 6.3 & 6.2 & 2.7 & 5.4 & 7.3 & 6.8 & 8.1 & 7.0 \\
\hline GDP deflator & 17.8 & 19.4 & 30.3 & 23.4 & 12.8 & 9.8 & 8.3 & 8.4 \\
\hline Consumer prices, period average & 16.7 & 18.0 & 45.0 & 24.7 & 13.5 & 10.5 & 9.0 & 8.8 \\
\hline Consumer prices, end-of-period & 10.0 & 27.6 & 48.7 & 15.0 & 12.0 & 9.0 & 9.0 & 8.5 \\
\hline \multicolumn{9}{|l|}{ External sector } \\
\hline Exports, f.o.b. (U.S.dollars) & 109.6 & 7.2 & -42.5 & 18.3 & 27.8 & 13.2 & 17.2 & 16.4 \\
\hline Imports, f.o.b. (U.S.\$ dollars) & 81.8 & 27.6 & -21.7 & 21.6 & 26.1 & 0.5 & 4.5 & 6.8 \\
\hline Export volume & 75.0 & -3.9 & 5.4 & 9.4 & 23.9 & 11.8 & 16.5 & 13.9 \\
\hline Import volume & 69.0 & 15.8 & -11.9 & 17.0 & 23.4 & -1.4 & 2.5 & 4.9 \\
\hline Terms of trade & 11.3 & 1.1 & -38.6 & 4.1 & 0.9 & -0.8 & -1.4 & 0.3 \\
\hline Nominal effective exchange rate ${ }^{1}$ & -15.5 & -11.7 & $\ldots$ & $\ldots$ & $\ldots$ & $\ldots$ & $\ldots$ & $\ldots$ \\
\hline \multirow[t]{2}{*}{ Real effective exchange rate ${ }^{1}$} & -3.1 & -0.7 & $\cdots$ & $\cdots$ & $\cdots$ & $\cdots$ & $\cdots$ & $\cdots$ \\
\hline & \multicolumn{8}{|c|}{$\begin{array}{c}\text { Annual change in percent of beginning-of-period broad money; } \\
\text { unless otherwise indicated) }\end{array}$} \\
\hline \multicolumn{9}{|l|}{ Money and credit } \\
\hline Broad money & 49.5 & 55.7 & 33.8 & 27.0 & $\ldots$ & $\ldots$ & $\ldots$ & $\ldots$ \\
\hline Net foreign assets & 30.8 & -6.0 & -6.9 & 38.6 & $\ldots$ & $\ldots$ & $\ldots$ & $\ldots$ \\
\hline Net domestic assets & 20.2 & 65.6 & 41.6 & -11.2 & $\ldots$ & $\ldots$ & $\ldots$ & $\ldots$ \\
\hline Domestic credit & 29.5 & 54.7 & 15.6 & 13.8 & $\ldots$ & $\ldots$ & $\ldots$ & $\ldots$ \\
\hline \multicolumn{9}{|l|}{ Of which: } \\
\hline Net credit to government & 10.3 & 11.3 & -10.6 & 0.0 & $\ldots$ & $\ldots$ & $\ldots$ & $\ldots$ \\
\hline \multirow[t]{2}{*}{ Credit to the private sector (annual percent change) } & 73.6 & 141.9 & 55.5 & 25.4 & $\ldots$ & $\ldots$ & $\ldots$ & $\ldots$ \\
\hline & \multicolumn{8}{|c|}{ (Percent of GDP; unless otherwise indicated) } \\
\hline \multicolumn{9}{|l|}{ Central government finance } \\
\hline Total government revenue & 14.8 & 18.5 & 18.0 & 17.9 & 19.0 & 20.2 & 20.8 & 21.2 \\
\hline Excluding signining bonus from the Sino-Congolese Cooperation Agreement & 14.8 & 18.5 & 15.7 & 17.9 & 19.0 & 20.2 & 20.8 & 21.2 \\
\hline Grants & 1.5 & 1.9 & 7.9 & 6.1 & 6.2 & 5.7 & 4.8 & 4.3 \\
\hline Total government expenditure ${ }^{2}$ & 18.8 & 22.7 & 29.1 & 36.2 & 35.3 & 34.6 & 32.1 & 30.8 \\
\hline Underlying fiscal balance (cash basis) & 0.8 & 0.9 & -1.9 & -0.5 & -0.6 & -0.4 & 0.4 & 0.4 \\
\hline Overall fiscal balance (payment order basis, incl. grants) & -2.5 & -2.3 & -3.2 & -12.2 & -10.1 & -8.7 & -6.4 & -5.3 \\
\hline Overall fiscal balance (cash basis, incl. grants) & -3.1 & -3.1 & -4.8 & -12.7 & -10.8 & -9.2 & -6.2 & -4.9 \\
\hline \multicolumn{9}{|l|}{ Investment and saving } \\
\hline Gross national saving & 16.7 & 6.1 & 7.0 & 5.8 & 5.6 & 7.4 & 11.2 & 14.4 \\
\hline Government & -1.0 & -0.5 & 1.0 & -0.1 & 1.8 & 2.9 & 3.8 & 4.2 \\
\hline Nongovernment & 17.7 & 6.5 & 6.0 & 5.9 & 3.8 & 4.4 & 7.4 & 10.2 \\
\hline Investment & 18.3 & 22.0 & 23.4 & 33.5 & 37.3 & 32.5 & 30.8 & 30.0 \\
\hline Government $^{3}$ & 2.4 & 3.7 & 9.3 & 18.0 & 17.9 & 17.1 & 15.1 & 13.8 \\
\hline Nongovernment & 15.9 & 18.2 & 14.1 & 15.5 & 19.4 & 15.4 & 15.7 & 16.2 \\
\hline \multicolumn{9}{|l|}{ Balance of payments } \\
\hline Exports of goods and services & 65.5 & 61.3 & 41.8 & 42.7 & 49.0 & 50.8 & 53.9 & 57.2 \\
\hline Imports of goods and services & 68.9 & 76.4 & 66.4 & 71.1 & 81.1 & 76.0 & 73.0 & 71.8 \\
\hline Current account balance, incl. transfers & -1.5 & -15.9 & -16.4 & -27.7 & -31.7 & -25.1 & -19.6 & -15.6 \\
\hline Current account balance, excl. transfers & -9.0 & -24.7 & -30.4 & -34.4 & -37.7 & -30.7 & -24.6 & -20.2 \\
\hline Current account balance, incl. transfers, after debt relief & 3.0 & -12.3 & -13.0 & -24.1 & -27.3 & -21.6 & -16.8 & -13.4 \\
\hline Gross official reserves (end-of-period, U.S.\$ millions of U.S. dollars) & 180.6 & 77.8 & 1,039 & 1,223 & 1,314 & 1,511 & 1,769 & 1,919 \\
\hline \multirow[t]{2}{*}{ Gross official reserves (weeks of nonaid-related imports of goods and services) } & 1.5 & 1.0 & 9.9 & 8.7 & 9.2 & 9.9 & 11.5 & 11.5 \\
\hline & & & is of U.S. do & ars; unless & therwise inc & licated) & & \\
\hline External public debt & & & & & & & & \\
\hline Total stock, including IMF ${ }^{4}$ & 13,425 & 13,149 & 12,785 & 4,329 & 5,386 & 6,434 & 6,882 & 7,761 \\
\hline Net present value (NPV) of debt ${ }^{5}$ & 10,820 & 10,729 & 10,603 & 4,106 & 5,255 & 6,248 & 6,599 & 7,326 \\
\hline NPV of debt (percent of exports of goods and services) ${ }^{5}$ & 264.5 & 189.7 & 175.5 & 73.8 & 98.9 & 100.8 & 90.9 & 88.0 \\
\hline Scheduled debt service & 739.6 & 891.2 & 815.5 & 252.1 & 346.6 & 430.4 & 355.7 & 350.8 \\
\hline Percent of exports of goods and services & 11.3 & 14.0 & 18.2 & 4.9 & 5.5 & 6.0 & 4.3 & 3.7 \\
\hline Percent of government revenue & 45.6 & 37.8 & 29.4 & 8.8 & 10.6 & 11.8 & 9.0 & 8.2 \\
\hline Exchange rate, (Congo franc per U.S. dollar) & & & & & & & & \\
\hline Period average & 516.0 & 563 & $\ldots$ & $\ldots$ & $\ldots$ & $\ldots$ & $\ldots$ & $\ldots$ \\
\hline End-of-period & 503.0 & 639 & $\ldots$ & $\ldots$ & $\ldots$ & $\ldots$ & $\ldots$ & $\ldots$ \\
\hline Memorandum item: & & & & & & & & \\
\hline Nominal GDP (billions of Congo francs) & 5,148 & 6,526 & 8,729 & 11,361 & 13,746 & 16,117 & 18,869 & 21,877 \\
\hline
\end{tabular}

Sources: Congolese authorities; and IMF staff estimates and projections.

${ }^{1}$ Change in annual average. Minus sign indicates depreciation.

2 Includes interest due before debt relief and expenditure financed by HIPC resources.

${ }^{3}$ Includes investment financed by resources released under the enhanced HIPC Initiative.

${ }^{4}$ End-of-period debt stock includes most of London Club debt (some US $\$ 1.2$ billion in 2008), which is expected to be bought back with deep discount grants from IDA, and accumulated arrears.

${ }^{5}$ Estimates and projections are based on the 2009 DSA and after HIPC Initiative interim relief assistance. Includes assistance beyond the terms of the enhanced HIPC 'Initiative granted by some Paris Club creditors. Exports are on a three-year backward moving average; projections assume DRC reaches the HIPC completion point during the first half of 2010. 
Table 2a. Democratic Republic of the Congo: Central Government Financial Operations, 2007-12

\begin{tabular}{|c|c|c|c|c|c|c|c|}
\hline & \multirow[t]{2}{*}{2007} & \multirow{2}{*}{$\frac{2008}{\text { Prel. }}$} & \multicolumn{2}{|c|}{2009} & 2010 & 2011 & 2012 \\
\hline & & & Prog. & Est. & \multicolumn{3}{|c|}{ Projections } \\
\hline & \multicolumn{7}{|c|}{ (Billions of Congo francs; unless otherwise indicated) } \\
\hline Total revenue and grants & 837.0 & $1,326.8$ & $2,363.6$ & $2,253.1$ & $2,729.2$ & $3,464.1$ & $4,172.9$ \\
\hline Total revenue & 761.0 & $1,205.3$ & $1,554.3$ & $1,565.7$ & $2,031.1$ & $2,605.0$ & $3,247.8$ \\
\hline Customs and excise & 278.1 & 427.1 & 542.5 & 555.1 & 805.7 & $1,025.8$ & $1,219.8$ \\
\hline Direct and indirect taxes & 259.9 & 430.7 & 557.0 & 557.0 & 733.7 & 982.4 & $1,353.9$ \\
\hline Petroleum (royalties and taxes) & 159.3 & 229.6 & 119.5 & 110.5 & 289.8 & 354.7 & 387.8 \\
\hline Administrative revenues & 63.8 & 118.0 & 335.2 & 343.0 & 202.0 & 242.2 & 286.2 \\
\hline Of which: signing bonus for joint venture with China & 0.0 & 0.0 & 195.2 & 199.0 & 0.0 & 0.0 & 0.0 \\
\hline Total grants & 76.0 & 121.5 & 809.4 & 687.4 & 698.1 & 859.1 & 925.1 \\
\hline Budget grants & 0.0 & 0.0 & 231.2 & 237.6 & 0.0 & 0.0 & 0.0 \\
\hline Project grants & 32.5 & 70.7 & 359.1 & 372.9 & 618.7 & 766.6 & 815.5 \\
\hline HIPC Initiative assistance ${ }^{1}$ & 43.5 & 50.8 & 219.1 & 76.9 & 79.5 & 92.5 & 109.6 \\
\hline Total expenditure & 966.7 & $1,478.7$ & $2,501.5$ & $2,543.1$ & $4,112.2$ & $4,852.3$ & $5,568.9$ \\
\hline Current expenditure & 800.5 & 1160.6 & $1,406.6$ & $1,420.0$ & 1857.0 & 2262.8 & 2712.8 \\
\hline Wages & 301.0 & 452.2 & 547.6 & 547.6 & 758.2 & 924.2 & $1,083.7$ \\
\hline Interest due & 184.1 & 203.8 & 281.1 & 294.4 & 345.0 & 370.3 & 381.8 \\
\hline Of which: on external debt & 155.4 & 169.5 & 248.2 & 261.5 & 308.9 & 303.4 & 307.9 \\
\hline Transfers and subsidies & 111.7 & 226.9 & 260.9 & 260.9 & 346.8 & 416.2 & 497.1 \\
\hline Subsidies & 6.2 & 15.7 & 15.0 & 15.0 & 31.5 & 26.9 & 28.5 \\
\hline Subordinated budgetary institutions & 9.3 & 9.2 & 9.8 & 9.8 & 21.1 & 26.3 & 31.7 \\
\hline Transfers to provinces & 49.7 & 125.0 & 142.6 & 142.6 & 185.6 & 224.5 & 263.3 \\
\hline Transfers to collection agencies & 46.6 & 74.8 & 91.4 & 91.4 & 97.2 & 124.6 & 157.3 \\
\hline Scholarships & $\ldots$ & 2.1 & 2.0 & 2.0 & 11.5 & 13.9 & 16.3 \\
\hline Goods and services & 203.7 & 277.7 & 317.1 & 317.1 & 407.0 & 552.0 & 750.2 \\
\hline Capital expenditure & 121.1 & 243.7 & 792.0 & 812.7 & $2,041.6$ & $2,453.8$ & $2,754.4$ \\
\hline Foreign-financed & 76.9 & 136.7 & 539.2 & 559.9 & $1,589.3$ & $1,853.9$ & $1,978.1$ \\
\hline Domestic-financed & 44.2 & 107.0 & 252.8 & 252.8 & 452.2 & 599.9 & 776.3 \\
\hline Government & 44.2 & 107.0 & 141.3 & 141.3 & 105.6 & 127.7 & 149.8 \\
\hline Provinces & 0.0 & 0.0 & 111.6 & 111.6 & 346.7 & 472.2 & 626.6 \\
\hline Exceptional expenditure ${ }^{2}$ & 45.0 & 74.4 & 296.4 & 303.9 & 185.2 & 101.4 & 61.4 \\
\hline Foreign-financed & 0.0 & 0.0 & 195.0 & 202.5 & 91.6 & 73.3 & 58.6 \\
\hline Domestic-financed & 45.0 & 74.4 & 101.4 & 101.4 & 93.6 & 28.1 & 2.8 \\
\hline Budget Reserve & 0.0 & 0.0 & 6.5 & 6.5 & 28.4 & 34.4 & 40.3 \\
\hline Overall fiscal balance (payment order basis) & -129.7 & -151.9 & -137.8 & -289.9 & $-1,383.0$ & $-1,388.2$ & $-1,396.0$ \\
\hline Underlying fiscal balance (payment order basis) ${ }^{3}$ & 71.6 & 107.2 & -61.6 & -53.7 & 2.3 & 11.3 & 26.2 \\
\hline Domestic fiscal balance $^{4}$ & 26.6 & 32.8 & -160.1 & -152.4 & -91.3 & -16.8 & 23.4 \\
\hline Change in arrears (increase $=+$ ) & -11.3 & -15.6 & -49.9 & -51.4 & -28.0 & -55.5 & -50.0 \\
\hline Central bank operational result & -19.9 & -35.8 & -74.4 & -74.4 & -34.7 & -34.7 & -34.7 \\
\hline Overall fiscal balance (cash basis, before interest rescheduling) & -160.8 & -203.3 & -262.2 & -415.7 & $-1,445.7$ & $-1,478.4$ & $-1,480.7$ \\
\hline Underlying fiscal balance (cash basis) & 40.5 & 55.8 & -185.9 & -179.5 & -60.4 & -78.9 & -58.5 \\
\hline Domestic fiscal balance & -4.5 & -18.6 & -284.5 & -278.2 & -154.0 & -106.9 & -61.3 \\
\hline Total financing & 160.8 & 203.3 & 262.2 & 415.7 & $1,445.7$ & $1,478.4$ & $1,480.7$ \\
\hline Domestic financing & 48.6 & 58.7 & -70.6 & -69.2 & 0.0 & 0.0 & 0.0 \\
\hline Banking system & 44.1 & 72.7 & -105.8 & -105.8 & 0.0 & 0.0 & 0.0 \\
\hline Nonbank & 4.4 & -14.0 & 0.0 & 0.0 & 0.0 & 0.0 & 0.0 \\
\hline Privatization receipts & $\ldots$ & 0.0 & 35.3 & 36.6 & $\ldots$ & $\ldots$ & $\ldots$ \\
\hline Foreign financing & 155.4 & 119.4 & 282.3 & 484.9 & $1,180.1$ & $1,224.5$ & $1,216.6$ \\
\hline Amortization due before debt relief & -202.8 & -249.7 & -420.1 & -346.5 & -237.8 & -297.2 & -349.1 \\
\hline Exceptional Financing & 0.0 & 0.0 & 827.0 & 0.0 & $1,497.7$ & 0.0 & 0.0 \\
\hline Budget loans & 0.0 & 0.0 & 0.0 & 0.0 & 0.0 & 0.0 & 0.0 \\
\hline Project loans & 60.9 & 51.8 & 398.3 & 413.6 & $1,090.8$ & $1,203.0$ & $1,261.4$ \\
\hline Debt relief & 39.5 & 42.8 & 27.8 & 62.6 & 327.2 & 318.8 & 304.2 \\
\hline Accumulation of external arrears (net, + increase) & 257.7 & 274.5 & -550.6 & 355.2 & $-1,497.7$ & 0.0 & 0.0 \\
\hline Residual financing gap/errors and omissions & -43.1 & 25.3 & 50.40 & 0.00 & 265.6 & 253.9 & 264.2 \\
\hline \multicolumn{8}{|l|}{ Memorandum items: } \\
\hline GDP (Billions of Congo francs) & 5,148 & 6,526 & 8,729 & 8,729 & 11,361 & 13,746 & 16,117 \\
\hline Underlying fiscal balance (cash basis; excl. oil revenues) ${ }^{3}$ & -118.8 & -173.8 & -305.4 & -290.1 & -350.1 & -433.5 & -446.3 \\
\hline Total wage bill & 301.0 & 452.2 & 547.6 & 547.6 & 758.2 & 924.2 & $1,083.7$ \\
\hline Central government wage bill & 301.0 & 452.2 & 547.6 & 367.0 & 516.9 & 628.8 & 737.3 \\
\hline Provincial government wage bill & $\ldots$ & 0.0 & 0.0 & 180.5 & 241.3 & 295.4 & 346.4 \\
\hline Total goods and services & 253.4 & 402.7 & 459.7 & 459.7 & 592.6 & 776.6 & $1,013.5$ \\
\hline Central government goods and services & 203.7 & 277.7 & 317.1 & 317.1 & 407.0 & 552.0 & 750.2 \\
\hline Provincial governments' non-wage expenditure & 49.7 & 125.0 & 142.6 & 142.6 & 185.6 & 224.5 & 263.3 \\
\hline Domestically financed spending & 765.5 & $1,223.9$ & $1,637.0$ & $1,638.5$ & $2,156.6$ & $2,677.6$ & $3,268.7$ \\
\hline
\end{tabular}

Sources: Congolese authorities; and IMF staff estimates and projections.

${ }^{1}$ Reflects revised calculation of HIPC Initiative assistance on the basis of the 2009 Debt Sustainability Analysis.

${ }^{2}$ Exceptional expenditure includes spending for the Demobilization, Disarmament, and Reintegration (DDR) program, and cost of the elections

${ }^{3}$ Underlying fiscal balance is defined as revenue (excluding signing bonus from the SCCA) minus expenditure (excluding interest on foreign debt, foreign-financed capital expenditure, and all exceptional spending).

${ }^{4}$ The domestic fiscal balance is defined as revenue (excluding the signing bonus from the SCCA) minus total expenditure (excluding interest on foreign debt, foreign-financed capital and exceptional expenditure).

\section{CInternational Monetary Fund. Not for Redistribution}


Table 2b. Democratic Republic of the Congo: Central Government Financial Operations, 2007-12

\begin{tabular}{|c|c|c|c|c|c|c|c|}
\hline & \multirow[t]{2}{*}{2007} & \multirow{2}{*}{$\frac{2008}{\text { Prel. }}$} & \multicolumn{2}{|c|}{2009} & 2010 & 2011 & 2012 \\
\hline & & & Prog. & Est. & \multicolumn{3}{|c|}{ Projections } \\
\hline & \multicolumn{7}{|c|}{ (Percent of GDP; unless otherwise indicated) } \\
\hline Total revenue and grants & 16.3 & 20.3 & 27.1 & 25.8 & 24.0 & 25.2 & 25.9 \\
\hline Total revenue & 14.8 & 18.5 & 17.8 & 17.9 & 17.9 & 19.0 & 20.2 \\
\hline Customs and excise & 5.4 & 6.5 & 6.2 & 6.4 & 7.1 & 7.5 & 7.6 \\
\hline Direct and indirect taxes & 5.0 & 6.6 & 6.4 & 6.4 & 6.5 & 7.1 & 8.4 \\
\hline Petroleum (royalties and taxes) & 3.1 & 3.5 & 1.4 & 1.3 & 2.6 & 2.6 & 2.4 \\
\hline Other & 1.2 & 1.8 & 3.8 & 3.9 & 1.8 & 1.8 & 1.8 \\
\hline Of which: Signing bonus from joint venture with China & $\ldots$ & 0.0 & 2.2 & 2.3 & $\ldots$ & $\ldots$ & $\ldots$ \\
\hline Total grants & 1.5 & 1.9 & 9.3 & 7.9 & 6.1 & 6.2 & 5.7 \\
\hline Budget grants & 0.0 & 0.0 & 2.6 & 2.7 & 0.0 & 0.0 & 0.0 \\
\hline Project grants & 0.6 & 1.1 & 4.1 & 4.3 & 5.4 & 5.6 & 5.1 \\
\hline HIPC Initiative assistance ${ }^{1}$ & 0.8 & 0.8 & 2.5 & 0.9 & 0.7 & 0.7 & 0.7 \\
\hline Total expenditure & 18.8 & 22.7 & 28.7 & 29.1 & 36.2 & 35.3 & 34.6 \\
\hline Current expenditure & 15.5 & 17.8 & 16.1 & 16.3 & 16.3 & 16.5 & 16.8 \\
\hline Wages & 5.8 & 6.9 & 6.3 & 6.3 & 6.7 & 6.7 & 6.7 \\
\hline Interest due & 3.6 & 3.1 & 3.2 & 3.4 & 3.0 & 2.7 & 2.4 \\
\hline Of which: On external debt & 3.0 & 2.6 & 2.8 & 3.0 & 2.7 & 2.2 & 1.9 \\
\hline Transfers and subsidies & 2.2 & 3.5 & 3.0 & 3.0 & 3.1 & 3.0 & 3.1 \\
\hline Subsidies & 0.1 & 0.2 & 0.2 & 0.2 & 0.3 & 0.2 & 0.2 \\
\hline Subordinated budgetary institutions & 0.2 & 0.1 & 0.1 & 0.1 & 0.2 & 0.2 & 0.2 \\
\hline Transfers to provinces & 1.0 & 1.9 & 1.6 & 1.6 & 1.6 & 1.6 & 1.6 \\
\hline Transfers to collection agencies & 0.9 & 1.1 & 1.0 & 1.0 & 0.9 & 0.9 & 1.0 \\
\hline Goods and servicees & 4.0 & 4.3 & 3.6 & 3.6 & 3.6 & 4.0 & 4.7 \\
\hline Capital expenditure & 2.4 & 3.7 & 9.1 & 9.3 & 18.0 & 17.9 & 17.1 \\
\hline Foreign-financed & 1.5 & 2.1 & 6.2 & 6.4 & 14.0 & 13.5 & 12.3 \\
\hline Domestic-financed & 0.9 & 1.6 & 2.9 & 2.9 & 4.0 & 4.4 & 4.8 \\
\hline Exceptional expenditure ${ }^{2}$ & 0.9 & 1.1 & 3.4 & 3.5 & 1.6 & 0.7 & 0.4 \\
\hline Foreign-financed & 0.0 & 0.0 & 2.2 & 2.3 & 0.8 & 0.5 & 0.4 \\
\hline Domestic-financed & 0.9 & 1.1 & 1.2 & 1.2 & 0.8 & 0.2 & 0.0 \\
\hline Budget Reserve & 0.0 & 0.0 & 0.1 & 0.1 & 0.3 & 0.3 & 0.3 \\
\hline Overall fiscal balance (payment order basis) & -2.5 & -2.3 & -1.6 & -3.3 & -12.2 & -10.1 & -8.7 \\
\hline Underlying fiscal balance (payment order basis) ${ }^{3}$ & 1.4 & 1.6 & -0.7 & -0.6 & 0.0 & 0.1 & 0.2 \\
\hline Domestic fiscal balance $^{4}$ & 0.5 & 0.5 & -1.8 & -1.7 & -0.8 & -0.1 & 0.1 \\
\hline Change in arrears (increase $=+$ ) & -0.2 & -0.2 & -0.6 & -0.6 & -0.2 & -0.4 & -0.3 \\
\hline Central bank operational result & -0.4 & -0.5 & -0.9 & -0.9 & -0.3 & -0.3 & -0.2 \\
\hline Overall fiscal balance (cash basis, before interest rescheduling) & -3.1 & -3.1 & -3.0 & -4.8 & -12.7 & -10.8 & -9.2 \\
\hline Underlying fiscal balance (cash basis) ${ }^{3}$ & 0.8 & 0.9 & -2.1 & -2.1 & -0.5 & -0.6 & -0.4 \\
\hline Domestic fiscal balance $^{4}$ & -0.1 & -0.3 & -3.3 & -3.2 & -1.4 & -0.8 & -0.4 \\
\hline Total financing & 3.1 & 3.1 & 3.0 & 4.8 & 12.7 & 10.8 & 9.2 \\
\hline Domestic financing & 0.9 & 0.9 & -0.8 & -0.8 & 0.0 & 0.0 & 0.0 \\
\hline Banking system & 0.9 & 1.1 & -1.2 & -1.2 & 0.0 & 0.0 & 0.0 \\
\hline Nonbank & 0.1 & -0.2 & 0.0 & 0.0 & 0.0 & 0.0 & 0.0 \\
\hline Privatization receipts & $\ldots$ & 0.0 & 0.4 & 0.4 & $\ldots$ & $\ldots$ & $\ldots$ \\
\hline Foreign financing & 3.0 & 1.8 & 3.2 & 5.6 & 10.4 & 8.9 & 7.5 \\
\hline Amortization due before debt relief & -3.9 & -3.8 & -4.8 & -4.0 & -2.1 & -2.2 & -2.2 \\
\hline Exceptional Financing & 0.0 & 0.0 & 9.5 & 0.0 & 13.2 & 0.0 & 0.0 \\
\hline Budget loans & 0.0 & 0.0 & 0.0 & 0.0 & 0.0 & 0.0 & 0.0 \\
\hline Project loans & 1.2 & 0.8 & 4.6 & 4.7 & 9.6 & 8.8 & 7.8 \\
\hline Debt relief & 0.8 & 0.7 & 0.3 & 0.7 & 2.9 & 2.3 & 1.9 \\
\hline Accumulation of external arrears & 5.0 & 4.2 & -6.3 & 4.1 & -13.2 & 0.0 & 0.0 \\
\hline Residual financing gap & -0.8 & 0.4 & 0.6 & 0.0 & 2.3 & 1.8 & 1.6 \\
\hline \multicolumn{8}{|l|}{ Memorandum items: } \\
\hline GDP (billions of Congo francs) & 5,148 & 6,526 & 8,729 & 8,729 & 11,361 & 13,746 & 16,117 \\
\hline Underlying fiscal balance (cash basis; excl. oil revenues) ${ }^{3}$ & -2.3 & -2.7 & -3.5 & -3.3 & -3.1 & -3.2 & -2.8 \\
\hline Total wage bill & 5.8 & 6.9 & 6.3 & 6.3 & 6.7 & 6.7 & 6.7 \\
\hline Central government wage bill & 5.8 & 6.9 & 6.3 & 4.2 & 4.5 & 4.6 & 4.6 \\
\hline Provincial government wage bill & $\ldots$ & $\ldots$ & 0.0 & 2.1 & 2.1 & 2.1 & 2.1 \\
\hline Total goods and services & 4.9 & 6.2 & 5.3 & 5.3 & 5.2 & 5.6 & 6.3 \\
\hline Central government goods and services & 4.0 & 4.3 & 3.6 & 3.6 & 3.6 & 4.0 & 4.7 \\
\hline Provincial governments' non-wage expenditure & 1.0 & 1.9 & 1.6 & 1.6 & 1.6 & 1.6 & 1.6 \\
\hline
\end{tabular}

Sources: Congolese authorities; and IMF staff estimates and projections.

${ }^{1}$ Reflects revised calculation of HIPC Initiative assistance on the basis of 2009 Debt Sustainability Analysis

${ }^{2}$ Exceptional expenditure includes spending for the Demobilization, Disarmament, and Reintegration (DDR) program, and cost of the elections.

${ }^{3}$ Underlying fiscal balance is defined as revenue (excluding signing bonus from the SCCA) minus expenditure (excluding interest on foreign debt, foreign-financed capital, and all exceptional spending).

${ }^{4}$ The domestic fiscal balance is defined as revenue (excluding the signing bonus from the SCCA) minus total expenditure (excluding interest on foreign debt, foreign-financed capital and exceptional expenditure) . 
Table 3a. Democratic Republic of the Congo: Monetary Survey, 2007-10

(At current exchange rates)

\begin{tabular}{|c|c|c|c|c|c|}
\hline & 2007 & 2008 & \multicolumn{2}{|c|}{2009} & \multirow{2}{*}{$\begin{array}{c}2010 \\
\text { Dec } \\
\text { Proj }\end{array}$} \\
\hline & Dec & Dec & $\begin{array}{l}\text { Sep } \\
\text { Prel }\end{array}$ & $\begin{array}{l}\text { Dec } \\
\text { Est. }\end{array}$ & \\
\hline & \multicolumn{5}{|c|}{ (Billions of Congo francs) } \\
\hline Net foreign assets & -172.6 & -211.0 & -219.8 & -279.7 & 235.8 \\
\hline Net domestic assets & 831.4 & 1252.4 & 1544.0 & 1668.2 & 1518.3 \\
\hline Domestic credit & 373.4 & 724.3 & 812.6 & 880.3 & 1065.1 \\
\hline Net credit to government & 176.2 & 248.9 & 199.0 & 143.1 & 143.1 \\
\hline Credit to the private sector & 195.2 & 472.3 & 593.7 & 734.1 & 920.3 \\
\hline Credit to parastatals & 1.9 & 3.1 & 20.0 & 3.1 & 1.7 \\
\hline Other items, net (including valuation change) & 458.1 & 528.1 & 731.4 & 787.8 & 453.2 \\
\hline Broad money (M2) & 641.2 & 998.4 & 1286.9 & 1335.5 & 1696.2 \\
\hline Narrow money (M1) & 300.3 & 392.5 & 411.5 & 436.8 & 530.5 \\
\hline Currency in circulation & 233.3 & 304.6 & 331.5 & 354.5 & 435.9 \\
\hline Demand deposits & 67.0 & 87.9 & 80.0 & 82.3 & 94.6 \\
\hline Quasi money & 341.0 & 606.0 & 875.3 & 898.8 & 1165.7 \\
\hline Time deposits in domestic currency & 2.5 & 1.3 & 3.4 & 4.7 & 13.5 \\
\hline Foreign currency deposits & 338.5 & 604.7 & 872.0 & 894.0 & 1152.2 \\
\hline \multirow[t]{2}{*}{ Import deposits } & 17.6 & 42.9 & 37.3 & 52.9 & 57.9 \\
\hline & \multicolumn{5}{|c|}{ (Year-on year change in percent) } \\
\hline Net foreign assets & 43.4 & -22.2 & -74.5 & -32.6 & 184.3 \\
\hline Net domestic assets & 11.6 & 50.6 & 50.0 & 33.2 & -9.0 \\
\hline Domestic credit & 51.3 & 94.0 & 53.1 & 21.5 & 21.0 \\
\hline Net credit to government & 33.4 & 41.2 & 36.3 & -42.5 & 0.0 \\
\hline Credit to the private sector & 73.6 & 141.9 & 55.6 & 55.5 & 25.4 \\
\hline Credit to parastatals & -11.8 & 63.4 & 540.4 & 0.0 & -45.6 \\
\hline Other items, net (including valuation change) & -8.1 & 15.3 & 46.7 & 49.2 & -42.5 \\
\hline Broad money (M2) & 49.5 & 55.7 & 46.6 & 33.8 & 27.0 \\
\hline Narrow money (M1) & 41.2 & 30.7 & 26.9 & 11.3 & 21.4 \\
\hline Currency in circulation & 28.2 & 30.6 & 27.6 & 16.4 & 23.0 \\
\hline Demand deposits & 117.6 & 31.2 & 23.9 & -6.4 & 14.8 \\
\hline Quasi money & 57.8 & 77.7 & 58.2 & 48.3 & 29.7 \\
\hline Time deposits in domestic currency & 483.0 & -47.8 & 80.1 & 262.0 & 185.9 \\
\hline Foreign currency deposits & 56.9 & 78.6 & 58.2 & 47.9 & 28.9 \\
\hline \multirow[t]{2}{*}{ Import deposits } & 54.0 & 144.0 & 44.5 & 23.3 & 9.4 \\
\hline & \multicolumn{5}{|c|}{ (Annual change in percent of beginning-of-period broad money) } \\
\hline Net foreign assets & 30.8 & -6.0 & -0.9 & -6.9 & 38.6 \\
\hline Net domestic assets & 20.2 & 65.6 & 29.2 & 41.6 & -11.2 \\
\hline Domestic credit & 29.5 & 54.7 & 8.8 & 15.6 & 13.8 \\
\hline Net credit to government & 10.3 & 11.3 & -5.0 & -10.6 & 0.0 \\
\hline Credit to the private sector & 19.3 & 43.2 & 12.2 & 26.2 & 13.9 \\
\hline Credit to parastatals & -0.1 & 0.2 & 1.7 & 0.0 & -0.1 \\
\hline Other items, net (including valuation change) & -9.4 & 10.9 & 20.4 & 26.0 & -25.1 \\
\hline Broad money (M2) & 49.5 & 55.7 & 28.9 & 33.8 & 27.0 \\
\hline Narrow money (M1) & 20.4 & 14.4 & 1.9 & 4.4 & 7.0 \\
\hline Currency in circulation & 12.0 & 11.1 & 2.7 & 5.0 & 6.1 \\
\hline Demand deposits & 8.4 & 3.3 & -0.8 & -0.6 & 0.9 \\
\hline Quasi money & 29.1 & 41.3 & 27.0 & 29.3 & 20.0 \\
\hline Time deposits in domestic currency & 0.5 & -0.2 & 0.2 & 0.3 & 0.7 \\
\hline Foreign currency deposits & 28.6 & 41.5 & 26.8 & 29.0 & 19.3 \\
\hline Import deposits & 1.4 & 4.0 & -0.6 & 1.0 & 0.4 \\
\hline \multicolumn{6}{|l|}{ Memorandum items: } \\
\hline Nominal GDP (billions of Congo francs) & 5,148 & 6,526 & $\ldots$ & 8,729 & $11,361.3$ \\
\hline Velocity (GDP/broad money) & 8.0 & 6.5 & $\ldots$ & 6.5 & 6.7 \\
\hline Foreign currency deposits (percent of M2) & 52.8 & 60.6 & 67.8 & 66.9 & 67.9 \\
\hline Foreign currency deposits (percent of total deposits) & 83.0 & 87.1 & 91.3 & 91.1 & 91.4 \\
\hline
\end{tabular}

Sources: Congolese authorities; and IMF staff estimates and projections. 
Table 3b. Democratic Republic of the Congo: Accounts of the Central Bank of the Congo, 2007-10 (At current exchange rates)

\begin{tabular}{|c|c|c|c|c|c|}
\hline & 2007 & 2008 & \multicolumn{2}{|c|}{2009} & \multirow{2}{*}{$\begin{array}{c}2010 \\
\text { Dec } \\
\text { Proj }\end{array}$} \\
\hline & Dec & Dec & $\begin{array}{l}\text { Sep } \\
\text { Prel }\end{array}$ & $\begin{array}{l}\text { Dec } \\
\text { Est. }\end{array}$ & \\
\hline & \multicolumn{5}{|c|}{ (Billions of Congo francs) } \\
\hline Net foreign assets & -333.3 & -390.1 & -620.0 & -515.4 & -80.4 \\
\hline Net domestic assets & 621.7 & 773.8 & 1027.5 & 974.2 & 640.1 \\
\hline Domestic credit & 206.6 & 295.2 & 298.0 & 230.5 & 231.1 \\
\hline Net credit to government & 198.7 & 272.4 & 232.9 & 166.6 & 166.6 \\
\hline Credit to the private sector & 3.485 & 3.0 & 7.1 & 3.0 & 3.0 \\
\hline Credit to parastatals & 0.0 & 0.0 & 0.0 & 0.0 & 0.0 \\
\hline Claims on deposit money banks & 4.4 & 19.7 & 58.0 & 60.9 & 61.4 \\
\hline Other items, net & 415.1 & 478.6 & 729.4 & 743.7 & 409.0 \\
\hline Of which: BTR & 32.9 & 47.0 & 56.9 & 81.9 & 98.8 \\
\hline Base money & 288.5 & 383.7 & 407.5 & 458.7 & 559.7 \\
\hline Narrow base money & 283.6 & 378.1 & 400.0 & 453.1 & 554.1 \\
\hline Currency in circulation & 243.8 & 320.3 & 352.9 & 367.5 & 453.9 \\
\hline In bank vaults & 10.5 & 15.7 & 21.4 & 13.0 & 18.0 \\
\hline Outside banks & 233.3 & 304.6 & 331.5 & 354.5 & 435.9 \\
\hline Deposits at the central bank & 38.7 & 56.2 & 43.8 & 84.1 & 98.6 \\
\hline Private sector deposits & 0.6 & 0.9 & 1.6 & 0.9 & 0.9 \\
\hline Parastatal deposits & 0.4 & 0.7 & 1.7 & 0.7 & 0.7 \\
\hline Foreign currency deposits & 1.9 & 2.7 & 3.5 & 2.7 & 2.7 \\
\hline \multirow[t]{2}{*}{ Import deposits } & 3.0 & 2.9 & 4.0 & 2.9 & 2.9 \\
\hline & \multicolumn{5}{|c|}{ (Year-on year change in percent) } \\
\hline Net foreign assets & 23.1 & -17.0 & -87.9 & -32.1 & 84.4 \\
\hline Net domestic assets & -3.6 & 24.5 & 55.9 & 25.9 & -34.3 \\
\hline Domestic credit & 15.5 & 42.9 & 46.6 & -21.9 & 0.3 \\
\hline Net credit to government & 21.8 & 37.1 & 28.0 & -38.8 & 0.0 \\
\hline Credit to the private sector & 279.1 & -13.1 & 36.2 & 0.0 & 0.0 \\
\hline Credit to parastatals & -48.5 & 0.0 & 0.0 & 0.0 & 0.0 \\
\hline Claims on deposit money banks & -70.2 & 347.7 & 258.9 & 208.7 & 1.0 \\
\hline Other items, net & -10.9 & 15.3 & 60.0 & 55.4 & -45.0 \\
\hline Base money & 36.5 & 33.0 & 23.8 & 19.6 & 22.0 \\
\hline Narrow base money & 38.9 & 33.3 & 23.2 & 19.8 & 22.3 \\
\hline Currency in circulation & 31.5 & 31.4 & 24.4 & 14.7 & 23.5 \\
\hline In bank vaults & 205.4 & 49.1 & -10.2 & -17.3 & 38.6 \\
\hline Outside banks & 28.2 & 30.6 & 27.6 & 16.4 & 23.0 \\
\hline Deposits at the central bank & 110.4 & 45.2 & 11.1 & 49.5 & 17.3 \\
\hline Private sector deposits & 426.3 & 48.5 & 312.0 & 0.0 & 0.0 \\
\hline Parastatal deposits & 101.8 & 62.6 & 39.8 & 0.0 & 0.0 \\
\hline Foreign currency deposits & -56.0 & 39.0 & 72.6 & 0.0 & 0.0 \\
\hline \multirow[t]{2}{*}{ Import deposits } & 5.0 & -1.7 & 58.7 & 0.0 & 0.0 \\
\hline & \multicolumn{5}{|c|}{ (Annual change in percent of beginning-of-period base money) } \\
\hline Net foreign assets & 47.5 & -19.7 & -59.9 & -32.7 & 94.8 \\
\hline Net domestic assets & -11.0 & 52.7 & 66.1 & 52.2 & -72.8 \\
\hline Domestic credit & 13.2 & 30.7 & 0.7 & -16.9 & 0.1 \\
\hline Net credit to government & 16.8 & 25.6 & -10.3 & -27.6 & 0.0 \\
\hline Credit to the private sector & 1.2 & -0.2 & 1.1 & 0.0 & 0.0 \\
\hline Credit to parastatals & 0.0 & 0.0 & 0.0 & 0.0 & 0.0 \\
\hline Claims on deposit money banks & -4.9 & 5.3 & 10.0 & 10.7 & 0.1 \\
\hline Other items, net & -24.1 & 22.0 & 65.4 & 69.1 & -73.0 \\
\hline Base money & 36.5 & 33.0 & 6.2 & 19.6 & 22.0 \\
\hline Narrow base money & 37.6 & 32.8 & 5.7 & 19.6 & 22.0 \\
\hline Currency in circulation & 27.7 & 26.5 & 8.5 & 12.3 & 18.8 \\
\hline In bank vaults & 3.4 & 1.8 & 1.5 & -0.7 & 1.1 \\
\hline Outside banks & 24.3 & 24.7 & 7.0 & 13.0 & 17.8 \\
\hline Deposits at the central bank & 9.6 & 6.1 & -3.2 & 7.3 & 3.2 \\
\hline Private sector deposits & 0.2 & 0.1 & 0.2 & 0.0 & 0.0 \\
\hline Parastatal deposits & 0.1 & 0.1 & 0.3 & 0.0 & 0.0 \\
\hline Foreign currency deposits & -1.2 & 0.3 & 0.2 & 0.0 & 0.0 \\
\hline Import deposits & 0.1 & 0.0 & 0.3 & 0.0 & 0.0 \\
\hline
\end{tabular}

Sources: Congolese authorities; and IMF staff estimates and projections. 
Table 4. Democratic Republic of the Congo: Balance of Payments Summary, 2007-14

\begin{tabular}{|c|c|c|c|c|c|c|c|c|}
\hline & 2007 & $\begin{array}{r}2008 \\
\text { Prel. }\end{array}$ & $\begin{array}{c}2009 \\
\text { Est. }\end{array}$ & \multicolumn{5}{|c|}{ Projections } \\
\hline & \multicolumn{8}{|c|}{ (Millions of dollars; unless otherwise indicated) } \\
\hline Current account & -153 & $-1,840$ & $-1,760$ & $-3,305$ & $-4,115$ & $-3,528$ & $-3,011$ & $-2,606$ \\
\hline Merchandise trade & 886 & -125 & $-1,467$ & $-1,907$ & $-2,330$ & $-1,620$ & -870 & -204 \\
\hline Exports, f.o.b. & 6,143 & 6,585 & 3,787 & 4,480 & 5,727 & 6,481 & 7,594 & 8,836 \\
\hline Of which: mining products & 5,108 & 5,422 & 3,076 & 3,639 & 4,788 & 5,494 & 6,550 & 7,569 \\
\hline Imports, f.o.b. & $-5,257$ & $-6,711$ & $-5,254$ & $-6,388$ & $-8,057$ & $-8,101$ & $-8,465$ & $-9,040$ \\
\hline Of which: aid-related imports & -739 & $-1,109$ & $-1,538$ & $-1,573$ & $-1,597$ & $-1,573$ & $-1,451$ & $-1,431$ \\
\hline Services & $-1,225$ & $-1,624$ & $-1,169$ & $-1,496$ & $-1,843$ & $-1,914$ & $-2,063$ & $-2,240$ \\
\hline Receipts & 392 & 522 & 695 & 618 & 631 & 660 & 682 & 708 \\
\hline Expenditure & $-1,618$ & $-2,146$ & $-1,864$ & $-2,115$ & $-2,474$ & $-2,573$ & $-2,745$ & $-2,947$ \\
\hline Of which: aid-related imports & -230 & -205 & -551 & -560 & -427 & -423 & -386 & -384 \\
\hline Income & -635 & $-1,321$ & -745 & -782 & -811 & -880 & -969 & $-1,069$ \\
\hline Receipts & 26 & 27 & 27 & 27 & 30 & 31 & 33 & 36 \\
\hline Expenditure & -661 & $-1,349$ & -772 & -809 & -841 & -911 & $-1,002$ & $-1,105$ \\
\hline Of which: interest payments ${ }^{1}$ & -367 & -366 & -326 & -297 & -291 & -272 & -258 & -243 \\
\hline Current transfers & 821 & 1,231 & 1,621 & 880 & 869 & 885 & 891 & 907 \\
\hline Of which: official aid & 740 & 1,019 & 1,499 & 805 & 781 & 784 & 772 & 763 \\
\hline Capital and financial account & 66 & 1,266 & 1,347 & 2,845 & 3,492 & 3,071 & 2,760 & 2,307 \\
\hline Capital account & 18 & 145 & 375 & 435 & 518 & 521 & 490 & 490 \\
\hline Official & 92 & 223 & 458 & 524 & 615 & 624 & 600 & 608 \\
\hline Private & -74 & -78 & -84 & -89 & -97 & -103 & -110 & -118 \\
\hline Financial account & 49 & 1,121 & 972 & 2,410 & 2,974 & 2,550 & 2,270 & 1,816 \\
\hline Official capital & -96 & -315 & -23 & 904 & 919 & 855 & 312 & 434 \\
\hline Gross disbursements & 294 & 102 & 513 & 1,148 & 1,136 & 1,099 & 945 & 931 \\
\hline Scheduled amortization ${ }^{2}$ & -390 & -417 & -536 & -243 & -217 & -244 & -273 & -299 \\
\hline Private capital (net) & 145 & 1,435 & 995 & 1,505 & 2,055 & 1,695 & 1,959 & 1,382 \\
\hline Of which: Foreign direct investment & 374 & 1,713 & 626 & 786 & 1,119 & 1,026 & 965 & 1,050 \\
\hline Other Private non-banking sector (net) ${ }^{3}$ & -229 & -277 & 369 & 719 & 936 & 669 & 993 & 332 \\
\hline Balance before errors and omissions & -87 & -574 & -413 & -461 & -623 & -457 & -251 & -299 \\
\hline Errors and omissions & -262 & 0 & 0 & 0 & 0 & 0 & 0 & 0 \\
\hline Overall balance & -349 & -574 & -413 & -461 & -623 & -457 & -251 & -299 \\
\hline Financing & -99 & 158 & -31 & $-1,972$ & -345 & -415 & -295 & -191 \\
\hline Net change in non-Fund arrears ${ }^{4}$ & 163 & 171 & 197 & $-1,576$ & 0 & 0 & 0 & 0 \\
\hline Net banking sector reserves (increase $=-$ ) & -262 & -13 & -228 & -397 & -345 & -415 & -295 & -191 \\
\hline Central bank & -199 & -52 & -244 & -347 & -263 & -304 & -295 & -191 \\
\hline Of which: net Fund credit & -62 & -137 & 51 & -164 & -172 & -107 & -37 & -41 \\
\hline Commercial banks & -63 & 39 & 16 & -49 & -82 & -111 & 0 & 0 \\
\hline Financing need before exceptional assistance & -448 & -416 & -444 & $-2,433$ & -968 & -873 & -546 & -490 \\
\hline Exceptional financing & 448 & 416 & 368 & 1,999 & 574 & 489 & 431 & 372 \\
\hline Consolidation of arrears & 0 & 0 & 0 & 1,576 & 0 & 0 & 0 & 0 \\
\hline Debt relief ${ }^{5}$ & 448 & 416 & 368 & 424 & 574 & 489 & 431 & 372 \\
\hline \multirow[t]{2}{*}{ Residual financing need (overfinancing $=+$ ) } & 0 & 0 & -76 & -433 & -394 & -384 & -116 & -118 \\
\hline & \multicolumn{8}{|c|}{ (Percent of GDP, unless otherwise indicated) } \\
\hline Memorandum items: & & & & & & & & \\
\hline Debt service after debt relief (percent of exports of goods and services) & 1.4 & 1.5 & 1.5 & 1.6 & 2.7 & 2.7 & 2.3 & 2.0 \\
\hline Current account balance ( including official transfers, but before debt relief) & -1.5 & -15.9 & -16.4 & -27.7 & -31.7 & -25.1 & -19.6 & -15.6 \\
\hline Current account balance (excluding official transfers and debt relief) & -9.0 & -24.7 & -30.4 & -34.4 & -37.7 & -30.7 & -24.6 & -20.2 \\
\hline Current account balance (including official transfers and debt relief) & 3.0 & -12.3 & -13.0 & -24.1 & -27.3 & -21.6 & -16.8 & -13.4 \\
\hline Current account balance (excluding official transfers, but including debt relief) & -4.5 & -21.1 & -27.0 & -30.8 & -33.3 & -27.2 & -21.8 & -18.0 \\
\hline Gross official reserves (in millions of U.S. dollars) & 180.6 & 77.8 & $1,039.3$ & $1,222.6$ & $1,314.1$ & $1,511.2$ & $1,768.9$ & $1,919.0$ \\
\hline Weeks of non-aid-related imports of goods and services & 1.5 & 1.0 & 9.9 & 8.7 & 9.2 & 9.9 & 11.5 & 11.5 \\
\hline
\end{tabular}

Sources: Congolese authorities; and IMF staff estimates and projections.

' Including interest due to the IMF.

Excluding repayments to the IMF.

${ }^{3}$ Including unrecorded transactions. The latter may be substantial given weaknesses in statistics.

${ }^{4}$ Mainly arrears to Paris Club creditors.

${ }^{5}$ Assumes HIPC Completion point and MDRI during the first half of 2010 
Table 5. The Democratic Republic of the Congo: Tentative Schedule of Disbursements and Reviews Under the PRGF Arrangement, 2009-12

\begin{tabular}{|c|c|c|}
\hline Date & Disbursement & Conditions \\
\hline December 11, 2009 & SDR 49.493 million & Executive Board Approval. \\
\hline May 31, 2010 & SDR 49.493 million & $\begin{array}{l}\text { Completion of first review, based on } \\
\text { observance of performance criteria at end- } \\
\text { December } 2009 \text {. }\end{array}$ \\
\hline December 31, 2010 & SDR 49.493 million & $\begin{array}{l}\text { Completion of second review, based on } \\
\text { observance of performance criteria at end- } \\
\text { June } 2010 \text {. }\end{array}$ \\
\hline May 31, 2011 & SDR 49.493 million & $\begin{array}{l}\text { Completion of third review, based on } \\
\text { observance of performance criteria at end- } \\
\text { December } 2010 \text {. }\end{array}$ \\
\hline December 31, 2011 & SDR 49.493 million & $\begin{array}{l}\text { Completion of fourth review, based on } \\
\text { observance of performance criteria at end- } \\
\text { June } 2011 \text {. }\end{array}$ \\
\hline May 31, 2012 & SDR 49.493 million & $\begin{array}{l}\text { Completion of fifth review, based on } \\
\text { observance of performance criteria at end- } \\
\text { December } 2011 \text {. }\end{array}$ \\
\hline December 15, 2012 & SDR 49.492 million & $\begin{array}{l}\text { Completion of sixth review, based on } \\
\text { observance of performance criteria at end- } \\
\text { June } 2012 \text {. }\end{array}$ \\
\hline
\end{tabular}


Table 6. Democratic Republic of the Congo: Indicators of Capacity to Repay the Fund, 2009-22

\begin{tabular}{|c|c|c|c|c|c|c|c|c|c|c|c|c|c|c|}
\hline & 2009 & 2010 & 2011 & 2012 & 2013 & 2014 & 2015 & 2016 & 2017 & 2018 & 2019 & 2020 & 2021 & 2022 \\
\hline \multicolumn{15}{|l|}{ Fund obligations based on existing credit } \\
\hline (In millions of SDRs) & 99.6 & 107.7 & 112.5 & 70.0 & 25.1 & 27.6 & 32.8 & 27.3 & 27.2 & 27.0 & 13.6 & 0.3 & 0.3 & 0.3 \\
\hline Principal & 97.3 & 105.4 & 110.7 & 68.7 & 24.0 & 26.7 & 32.0 & 26.7 & 26.7 & 26.7 & 13.3 & 0.0 & 0.0 & 0.0 \\
\hline Charges and interest ${ }^{1}$ & 2.3 & 2.4 & 1.8 & 1.3 & 1.1 & 1.0 & 0.8 & 0.7 & 0.5 & 0.4 & 0.3 & 0.3 & 0.3 & 0.3 \\
\hline \multicolumn{15}{|l|}{ Fund obligations based on prospective credit ${ }^{2}$} \\
\hline (In millions of SDRs) & 0.0 & 0.5 & 1.0 & 1.5 & 1.7 & 1.7 & 16.6 & 36.3 & 55.8 & 70.4 & 70.0 & 54.8 & 34.8 & 14.9 \\
\hline Principal & 0.0 & 0.0 & 0.0 & 0.0 & 0.0 & 0.0 & 14.9 & 34.7 & 54.4 & 69.3 & 69.3 & 54.4 & 34.6 & 14.9 \\
\hline Charges and interest & 0.0 & 0.5 & 1.0 & 1.5 & 1.7 & 1.7 & 1.7 & 1.6 & 1.4 & 1.1 & 0.7 & 0.4 & 0.2 & 0.0 \\
\hline \multicolumn{15}{|l|}{ Total obligations on existing and prospective credit ${ }^{1}$} \\
\hline (In millions of SDRs) & 99.6 & 108.2 & 113.5 & 71.4 & 26.8 & 29.4 & 49.4 & 63.6 & 83.0 & 97.4 & 83.6 & 55.1 & 35.1 & 15.2 \\
\hline (In millions of U.S. dollars) & 152.7 & 168.4 & 176.3 & 110.9 & 41.6 & 45.5 & 76.4 & 98.4 & 128.5 & 150.8 & 129.5 & 85.3 & 54.3 & 23.5 \\
\hline In percent of exports of goods and NFS & 3.4 & 3.3 & 2.8 & 1.5 & 0.5 & 0.5 & 0.7 & 0.8 & 1.0 & 1.1 & 0.9 & 0.5 & 0.3 & 0.1 \\
\hline In percent of GDP & 1.4 & 1.4 & 1.4 & 0.8 & 0.3 & 0.3 & 0.4 & 0.5 & 0.6 & 0.7 & 0.5 & 0.3 & 0.2 & 0.1 \\
\hline In percent of quota & 18.7 & 20.3 & 21.3 & 13.4 & 5.0 & 5.5 & 9.3 & 11.9 & 15.6 & 18.3 & 15.7 & 10.3 & 6.6 & 2.8 \\
\hline In percent of gross international reserves & 14.7 & 13.8 & 13.4 & 7.3 & 2.4 & 2.4 & 3.9 & 4.8 & 5.8 & 6.1 & 4.8 & 2.9 & 1.7 & 0.7 \\
\hline \multicolumn{15}{|l|}{ Fund credit outstanding (end-period) } \\
\hline In millions of SDRs & 510.2 & 503.8 & 492.1 & 522.4 & 498.4 & 471.7 & 424.9 & 363.6 & 282.5 & 186.6 & 103.9 & 49.5 & 14.9 & 0.0 \\
\hline In millions of U.S. dollars & 781.7 & 784.2 & 764.6 & 811.1 & 773.2 & 730.5 & 657.9 & 563.0 & 437.4 & 288.9 & 160.9 & 76.6 & 23.0 & 0.0 \\
\hline In percent of exports of goods and NFS & 17.3 & 15.3 & 12.0 & 11.3 & 9.3 & 7.6 & 6.1 & 4.6 & 3.3 & 2.0 & 1.1 & 0.5 & 0.1 & 0.0 \\
\hline In percent of GDP & 7.3 & 6.6 & 5.9 & 5.8 & 5.0 & 4.4 & 3.6 & 2.9 & 2.1 & 1.3 & 0.7 & 0.3 & 0.1 & 0.0 \\
\hline In percent of quota & 95.7 & 94.5 & 92.3 & 98.0 & 93.5 & 88.5 & 79.7 & 68.2 & 53.0 & 35.0 & 19.5 & 9.3 & 2.8 & 0.0 \\
\hline In percent of total external debt & 6.1 & 5.1 & 4.5 & 4.4 & 4.0 & 3.6 & 3.1 & 2.6 & 2.0 & 1.3 & 0.8 & 0.4 & 0.1 & 0.0 \\
\hline \multirow{2}{*}{ In percent of gross international reserves } & 75.2 & 64.1 & 58.2 & 53.7 & 43.7 & 38.1 & 33.5 & 27.2 & 19.6 & 11.6 & 0.1 & 0.0 & 0.0 & 0.0 \\
\hline & \multicolumn{14}{|c|}{ (Millions of U.S. dollars, unless otherwise indicated) } \\
\hline \multicolumn{15}{|l|}{ Memorandum items } \\
\hline Exports of goods and services (in millions of U.S. dollars) & 4,509 & 5,126 & 6,388 & 7,172 & 8,310 & 9,580 & 10,853 & 12,204 & 13,236 & 14,167 & 15,160 & 16,083 & 16,988 & 17,911 \\
\hline Quota (in millions of SDRs) & 533 & 533 & 533 & 533 & 533 & 533 & 533 & 533 & 533 & 533 & 533 & 533 & 533 & 533 \\
\hline Gross international reserves (in millions of U.S. dollars) & 1,039 & 1,223 & 1,314 & 1,511 & 1,769 & 1,919 & 1,965 & 2,069 & 2,233 & 2,491 & 2,684 & 2,908 & 3,144 & 3,403 \\
\hline
\end{tabular}

Sources: IMF staff estimates and projections

${ }^{1}$ Upon the effectiveness of the ammendments to the PRGT instrument, there is a temporary interest waiver through end-2011 on PRGF outstanding obligations and drawings.

${ }^{2}$ Includes prospective disbursement under the PRGF of SDR 346.45 million. 
Table 7. The Democratic Republic of the Congo: Progress Status of HIPC Completion Point Triggers (As of October 31, 2009)

Triggers

\section{PRSP}

Completion of a full PRSP through a participatory process and its implementation for one year, duly documented in the DRC's annual progress report.

\section{Macroeconomic stability}

Continued maintenance of macroeconomic stability after reaching the decision point, as evidenced by satisfactory performance in a program supported by an arrangement under the IMF's PRGF.

3. Use of budgetary savings resulting from enhanced HIPC Initiative-related debt service relief during the interim period for poverty-related expenditures in accordance with the I-PRSP, with supporting documentation satisfactory to the staffs of IDA and the IMF.

\section{Public expenditure management}

(a) Implementation of a modernized budget-execution system, providing information from commitment to payment, and making it possible to monitor of arrears;

\section{Progress status}

DRC's full PRSP (2006-08) was (a) completed through a participatory process, (b) adopted by the government in July 2006, (c) endorsed by the democratically-elected government in March 2007, and (d) submitted to the Bank and the IMF. A priority action plan (PAP1) for the implementation of the PRSP (July 2007-December 2008) was adopted in July 2007 and an updated priority action plan (PAP2) for 2009-10 was adopted in 2009. Monitoring of the PRSP implementation suggests that little progress has been made in the social sectors and that many PRSP objectives will not be met. The government has decided to extend the PRSP through 2010, due to early delays in the beginning of its implementation. Preparation of a new PRSP (2011-15) began in June 2009.

After unsatisfactory performance on the first PRGF, the government embarked on a series of staff monitored programs (SMPs) from April 2006 through June 2009. Satisfactory completion of the first review of a new PRGF-supported program will be necessary.

Initially a special HIPC account was set up but it has not been used in recent years. The authorities plan to assess poverty-related expenditure as defined in the PRSP in comparison with other expenditures. The results of this approach are expected in early 2010.

(a) A new budget-execution system has been introduced in 2003 provides a reasonable amount of information on expenditure tracking (Chaine de la dépense). Budget execution reports (États de suivi budgétaire - ESB) are produced although they need improvements. Additionally, there are considerable delays in producing ESB harmonized with the state financial operations table (Tableau des opérations financières de l'État - TOFE). Sectoral budget execution reports are prepared by line ministries and sent to the Ministry of Budget but are not exhaustive. 
Table 7. The Democratic Republic of the Congo: Progress Status of Trigger for

Reaching the HIPC Completion Point (Continued)

Triggers

(b) adoption and implementation of a double-entry government accounting system and a new chart of accounts; and

(c) production of quarterly budget execution reports using economic, administrative, and functional classifications.

\section{Progress status}

(b) The double-entry system is in place. Staff have been trained, equipment purchased, and software developed. However, treasury accounts produced from the system are not exhaustive or fully reliable.

(c) A new classification has been adopted. Budgetary reports are produced using economic, administrative, and functional classifications, but with a lag of two to three months.

\section{Governance and service delivery in priority sectors}

(a) Completion of a budget-tracking exercise of health, education, rural development and infrastructure expenditure, consisting of (i) monitoring the execution of poverty-related expenditure; (ii) evaluation by user groups of the quality of related public services, and (iii) evaluation by serviceproviders of constraints to effective provision; and

(b) Adoption and implementation of a new procurement code and key implementing decrees. (a) The Treasury monitors poverty-related public expenditures in all sectors, using the new budget classifications. The evaluation by user groups of health clinics and schools and the evaluation by service providers in education and health were conducted in 2005 through an Institutional and Governance Review with World Bank support. Another survey of users on the quality of service delivery was launched in April 2009, with results expected in December 2009.

(b) The draft procurement code and its implementing laws were adopted by the government and in December 2008 submitted to Parliament which is expected to be adopted by year-end 2009. Institutions to operationalize the new code will be established.

The country health status report (RESP) was issued in May 2005 and led to the completion of the Health Sector Strategy in March 2006. This strategy will be updated during late in 2009.

The Status Report on the National Education System was finalized in September 2004. An outline of the sector strategy was drafted in 2006 and integrated into the full PRSP. Technical work to identify priorities for reform of the sector was conducted by independent commissions and finalized in 2008. The World Bank is currently reviewing the draft strategy for the primary and secondary education subsector. A note on the development on higher education strategy is expected to be completed by April 2010.

An Agriculture Sector Strategy that was finalized in September 2007 has yet to be validated and adopted. 
Table 7. The Democratic Republic of the Congo: Progress Status of Trigger for Reaching the HIPC Completion Point (Concluded)

Triggers

\section{Debt management}

Installation and full activation of a computerized debtrecording system, covering public and publiclyguaranteed debt that can (a) produce monthly debtservice projections, and incorporate actual disbursement and debt-service payment execution data; (b) produce advance monthly debt-service projections that will be published quarterly; and (c) support the centralization of debt information into a single center.
Progress status
Debt management software (SYGADE) was installed, external debt outstanding recorded, and the implementation program, including the training of relevant staff for debt management, completed. In 2008 the OGEDEP issued the first statistical report on public debt as of end-December 2007 using

SYGADE. The final report was due in April 2009 but has not yet been released. A Prime Ministerial decree strengthening the role of OGEDEP as the single center for debt information was signed in February 2008.

The related action plan for OGEDEP was validated by the Minister of Finance in May 2008, but implementation is yet to be effected.

Sources: World Bank and IMF staff based on information from Congolese authorities. 


\title{
APPENDIX I \\ TRANSLATED FROM FRENCH
}

\section{Democratic Republic OF THE CONGo LETTER OF INTENT}

Kinshasa, November 30, 2009

\author{
Mr. Dominique Strauss-Kahn \\ Managing Director \\ The International Monetary Fund \\ Washington, D.C. 20431 \\ United States of America
}

\section{Mr. Strauss-Kahn:}

As you know, since 2001 the Democratic Republic of the Congo has made progress on both the political and economic fronts. Indeed, during the period, the government has taken important steps toward establishing democracy, restoring peace, and fostering national reconciliation. On the economic front, prudent policies and reforms helped rekindle economic growth and tame hyperinflation.

The main obstacles that continue to affect economic recovery, despite progress in the pacification of the eastern provinces, are the continued periodic episodes of instability created by certain pockets of resistance. This situation has slowed down the implementation of our development agenda and put a severe burden on our limited budgetary resources. Economic recovery is also constrained by the lack to adequate infrastructure: dilapidated roads and railways, insufficient social infrastructure especially hospitals and schools, with negative consequences for human capital.

Most recently, despite the difficult circumstances brought on by the global financial crisis, we have persevered in implementing sound policies in the context of our economic program for the period January-June 2009 which was monitored by IMF staff. Notwithstanding the significant revenue shortfalls caused by weak mining activity and the high levels of securityrelated spending on account of the conflict in the eastern provinces, the government was able to abstain from recourse to central bank financing while building up its gross foreign reserves, broadly in line with the objectives of the staff monitored program. This was greatly facilitated by the assistance provided by the IMF through the Rapid-Access Component of its Exogenous Shocks Facility and the catalytic effect it had on generating emergency financial assistance from other development partners, including the World Bank, the African Development Bank, the European Commission, and bilateral donors including Belgium. 
Together, these helped reduce macroeconomic pressures going into the second half of the year.

The government's economic program for 2009-12 aims to address the challenges facing the country in line with our current Poverty Reduction and Growth Strategy, which we have extended through the end of 2010. Further, our second-generation strategy for 2011-15 is currently under preparation. Our program focuses on enhancing macroeconomic stability, increasing investment in physical and human capital formation, and the implementation of structural reforms to improve domestic resource mobilization, strengthen public financial management, enhance central bank independence, and boost our economy's productive capacity. These policies and reforms are set out in the attached Memorandum of Economic and Financial Policies (MEFP) for 2009-12 and accompanying Matrix of Structural Reforms. We also attach the Memorandum of Technical Understandings (TMU) that provides the modalities for monitoring program implementation.

I am pleased to inform you that we made significant progress in addressing earlier concerns over the debt sustainability implications of Sino-Congolese Cooperation Agreement involving large mining and infrastructure projects critical to our country's development. We agreed with our Chinese partners to remove the government guarantee on the financing of the mining component (US\$3.2 billion) and cancelled the second phase of infrastructure financing (US\$3 billion). This allowed us to limit the government guaranteed borrowing under the Agreement to strictly the US\$3 billion infrastructure component, which is highly concessional (i.e., grant element of 42-46 percent depending on whether the signing bonus is taken into account).

In this context, the Democratic Republic of the Congo is requesting IMF financial support under the Poverty Reduction and Growth Facility (PRGF) during the period July 2009-June 2012 in an amount of SDR 346.45 (or 65 percent) of quota. In addition, we request the resumption of interim relief under the enhanced HIPC initiative in the amount of SDR 45.66 million (20 percent of the total HIPC commitment) to help us cover part of the principal payments to the IMF falling due during the twelve month period from December 2009. IMF support will complement additional budget and balance of payments support being provided by other development partners, including the World Bank.

The government believes that the policies and reforms set forth in the attached MEFP are adequate to achieve our program's objectives. Nevertheless, we are committed to take any further measures that may prove necessary for achieving the program objectives. During the period covered by the three-year arrangement, the government will consult with the staff of the IMF on the adoption of any additional measures deemed to be appropriate.

The Government will provide the IMF with any information it may request for monitoring the implementation of the program's economic and financial policies. Furthermore, the government will conduct with the IMF a mid-term review of the first year of the program (July 2009-June 2010) no later than end-June 2010 and a 
subsequent review of the annual program (January 2010-June 2010) no later than endDecember 2010. The government intends to make the contents of this letter, the attached MEFP, the TMU, and the related IMF staff report available to the public. Therefore, it authorizes the Fund to publish these documents on its website once the Executive Board approves the PRGF arrangement and in the context of the subsequent reviews.

Sincerely yours,

Adolphe Muzito
Prime Minister

Attachments: Memorandum of Economic and Financial Policies

Technical Memorandum of Understanding 


\section{APPENDIX I \\ ATtaChMEnT I \\ TRANSLATED FROM FRENCH \\ DeMOCRATIC REPUblic OF THE CONGO \\ Memorandum of ECONOMiC ANd Financial Policies, 2009-12}

Kinshasa, November 30, 2009

\section{INTRODUCTION}

1. This Memorandum describes the economic and financial policies as well as the structural reforms that the government intends to implement in its program supported by an arrangement under the Poverty Reduction and Growth Facility (PRGF) of the International Monetary Fund (IMF) for the period July 2009 to June 2012. Through this program, the government is pursuing efforts to reestablish the conditions for lasting political, economic, and social stability. In particular, the proposed policies and reforms are aimed to address the legacy of decades of poor economic management, armed conflicts, corruption, and disregard for the rule of law.

2. The government's three-year program supported by the PRGF arrangement is based on the Poverty Reduction and Growth Strategy (PRGS) paper drafted in 2006 in the context of a largely participatory approach supported by the democratically elected government. The strategy is based on five pillars: (i) promote peace and good governance; (ii) consolidate macroeconomic stability and promote economic growth; (iii) improve access to social services; (iv) combat HIV/AIDS; and (v) promote proactive community involvement. Given that these objectives have yet to be realized, the government decided to extend its PRGS through December 2010, supported by a Priority Action Plan (PAP) which seeks to correct the weaknesses identified in the July 2008 PRGS progress report. The weaknesses included delays in implementing public financial management reforms, unexpected security-related spending, poor revenue mobilization, and weak institutional and human capacities. During this period, the government will prepare its second generation PRGS covering the period 2011-15 in a participatory manner.

\section{OVERVIEW}

3. The government has made considerable progress on the social and political fronts. Since 2003, the political transition has paved the way for democratization, restoration of peace, and reunification of the national territory with assistance from the international community, in particular through the deployment of a United Nations peacekeeping and institutional-building mission (MONUC).

4. On the economic side, the government successfully implemented its interim economic program for the period June 2001 to March 2002 that was monitored by IMF staff (SMP), which ended a long period of hyperinflation and led to the Government's Economic Program (PEG) being supported by a PRGF arrangement in April 2002. 
5. At the same time, the government prepared its Interim Poverty Reduction and Growth Strategy paper (I-PRGS). The priorities of the I-PRGS paper were restoration and reinforcement of peace, macroeconomic stabilization, and involvement of communities and civil society. The I-PRGS paper also identified ways in which new resources to finance the strategy could be generated. The satisfactory execution of the PEG underpinned by I-PRGS paper enabled the Democratic Republic of the Congo (DRC) to reach Decision point under the Heavily Indebted Poor Countries (HIPC) Initiative in July 2003 and obtain interim debt relief. The implementation of the PRGF-supported program and interim debt relief allowed the Congolese economy, for the first time in a decade, to experience positive rates of growth and to reduce inflation to below 10 percent in 2003-04.

\section{The momentum of the reforms proved difficult to sustain on account of the} continued conflict in the eastern provinces and the preparations for the first national election in 40 years. As a result, the macroeconomic program went off track and certain performance criteria were not observed, resulting in the sixth and final PRGF review not being concluded prior to the expiration of the arrangement and the suspension of the program.

7. Since 2006, the government has been implementing an IMF staff-monitored program that has been extended several times after yielding mixed results. The escalation of the conflict in the eastern provinces made public financial management difficult and led to significant security-related expenditure overruns that were difficult to offset with other fiscal measures. In this context, the implementation of the PRGS was less satisfactory than foreseen.

\section{The DRC is facing major post-conflict challenges of reconstruction and} rehabilitation of its dilapidated infrastructure, while maintaining macroeconomic stability and ensuring debt sustainability. The continued presence and military activities of rebel forces have set back the implementation of the government's development agenda and put a strain on its limited budgetary resources. Economic recovery was hampered by nonexistent or dilapidated basic infrastructure (roads, railways, hospitals, and schools). These factors constrain productivity and increase the cost of doing business, thereby reducing competitiveness. The government therefore aims at achieving its dual objective of rebuilding the country's infrastructure and obtaining significant debt relief through the HIPC Initiative and the Multilateral Debt Relief Initiative (MDRI).

\section{RECENT ECONOMIC DEVELOPMENTS (2008-09)}

9. The DRC was affected by the escalation of the conflict in the eastern provinces and the negative impact of exogenous shocks on the country's terms of trade following the onset of the global financial crisis. On the domestic front, the search for a lasting solution to the security problems in the eastern region of the country resulted in the holding of a Conference on Peace, Security, and Development of the Great Lakes Region in January 2008. However, the conference failed to reduce tensions and conflict reemerged in October 2008, thus jeopardizing the progress made since the end of the political transition 
and the establishment of the new democratic order. Through diplomatic efforts, the joint DRC-Rwanda and DRC-Uganda armed forces set out to dismantle the pockets of resistance created by Rwandan Hutu rebels (FDLR) and Ugandan rebels (LRA) in early 2009. These events resulted in significant unforeseen budgetary expenditures. Given the urgent and binding nature of these expenditures, the government at times had to execute them outside the expenditure chain. At the same time, the reduction in world demand and the sharp decline in export prices markedly reduced mining revenues. The combination of these endogenous and exogenous shocks widened the underlying fiscal deficit, increased net credit to government (NCG) from the banking sector, and reduced gross foreign reserves to less than one week of nonaid imports.

10. The global financial crisis weakened macroeconomic performance. In 2008, real GDP growth slowed to 6.2 percent (as opposed to a projected 10 percent) owing to the slump in mining sector activity. Inflation, which was at 27.6 percent at end-2008, peaked at over 55 percent in the first quarter of 2009, subsided in May and June, but started to rise in July and reached 54 percent in September in response to periodic strong increases in base money. At the same time, the Congolese franc depreciated from about CF 555 per U.S. dollar in September 2008 to about CF 880 per U.S. dollar at end October 2009.

11. The government implemented a stabilization program during the first half of 2009 . It reduced borrowing from the central bank notwithstanding weak revenue performance. This, combined with a tight monetary policy, helped reduce inflation and stabilize the exchange rate. Despite these efforts, the impact of the global financial crisis, the continuation of conflict in the eastern provinces, and the need for the central bank to address liquidity problems regarding certain commercial banks resulted in deviations from the targets under our SMP.

12. Government implemented its economic program satisfactorily through the third quarter of 2009, but expenditure pressures have increased in the fourth quarter. All prior actions and quantitative benchmarks through September were observed. However, there was a large build-up of expenditure commitments in September and October reflecting unavoidable security-related spending and cost overruns on the Southern African Development Community (SADC) and Economic Community of Central African States (CEEAC) regional conference hosted by the DRC. The government has already initiated measures to ensure that program's fiscal objectives are met.

13. The government made progress in resolving the earlier cases of misreporting to the IMF. Up to end April 2008, a large number of government accounts held at the BCC and the commercial banks limited the transparency of government operations. Furthermore, the government did not always observe proper procedures regarding the payment by the central bank of urgent government spending, which led the misreporting of data to IMF staff. From May 2008, to remedy this problem, the government ordered an audit of all its accounts, including those related to urgent spending. Moreover, in May 2008 it implemented new procedures to improve the management and transparency of urgent spending. However, delays were experienced in implementing these procedures. To further strengthen urgent 
spending management, the government adopted an inter-ministerial decree on December 29, 2008, that defined the scope for using urgent spending procedures. To date, the government successfully and transparently applied the new procedures and all payments on urgent government spending were in accordance with the December 2008 decree.

14. Following the international financial crisis, the government requested the IMF and other development partners to provide urgent balance of payments and budget support. In response, the IMF granted approximately US\$200 million through the Rapid Access Component of its Exogenous Shocks Facility. The World Bank granted US\$100 million, disbursed in multiple tranches starting in March 2009, while the African Development Bank (AfDB) disbursed about US\$97 million. Thanks to this support, international reserves rose to US\$250 million at end-July 2009. Other foreign partners, including the European Commission and Belgium, are providing emergency assistance to the DRC.

15. In order to rebuild the country's economic and social infrastructure, the government signed a cooperation agreement with a consortium of Chinese enterprises, which was amended in October 2009 to be consistent with debt sustainability. The agreement includes a government guarantee on US\$3 billion of financing for infrastructure development. The repayment of debt service associated with the financing will be covered by the profits of a joint venture between GECAMINES (a Congolese state-owned company) and the Chinese enterprises, and the government guarantee could only be invoked in the event that the joint venture fails to repay the entire debt service 25 years after its legal incorporation (i.e., 2034). In this context, the government believes that the deal is consistent with debt sustainability and the level of concessionality expected under a PRGF-supported program.

\section{Medium-term Economic ANd Financial Policies}

16. The government is implementing its medium-term economic and financial policies for the period July 2009 to June 2012 against the backdrop of an improved security outlook in the eastern provinces. Relations with neighboring countries are being normalized. Nonetheless, the presence of foreign-armed groups, which continue to terrorize local populations, and the integration of former rebel elements into the army, continue to impose large security-related budget and humanitarian costs.

\section{A. Macroeconomic framework}

17. The macroeconomic objectives are as follows: (i) achieving an average growth rate of 6.5 percent in 2009-12, sustained by a recovery of mining sector activity and infrastructure projects; (ii) reducing end-period inflation to 9 percent by 2012 supported by the implementation of prudent monetary and fiscal policies; and (iii) maintain gross foreign reserves to the equivalent of about 10 weeks of nonaid imports by the end of the program period. The external current account deficit (excluding grants) is projected to increase over the medium term to an average of 34 percent of GDP, owing mainly to a sharp increase in investment related imports. Exports will gradually recover from the adverse effects of the global financial crisis as world demand improves. The external current deficit will be financed by foreign direct investment and development assistance, including debt relief. 


\section{B. Fiscal policy}

18. Medium-term fiscal policy is geared to avoiding government recourse to central bank financing. The domestic fiscal deficit (cash basis) is targeted to decline from 3.3 percent of GDP in 2009 to 0.4 percent of GDP in 2012. ${ }^{9}$ Domestic revenue (excluding the signing bonus under the Sino-Congolese Cooperation Agreement) is projected to increase from about 15.7 percent of GDP in 2009 and to 20 percent of GDP in 2012, while domestically financed expenditure (including exceptional spending) will increase from 17.5 percent of GDP in 2009 to about 20 percent of GDP in 2012.

\section{The government is committed to implement a prudent expenditure policy.}

Regarding current spending, the policy of standardizing public sector wages of central government and provinces in 2010 will be maintained, with a wage bill capped at 6.7 percent of GDP over the medium term. This objective will be achieved by improving the reliability of the computerized payroll procedure and the rationalization of civil service staffing. The program will provide realistic allocations for spending on goods and services taking into account the significant increase in public investment. Over the medium term, domestically financed investment will increase from about 2.9 percent of GDP to about 5 percent of GDP. Foreign-financed investment will rise from about 6 percent of GDP in 2009 to about 12 percent in 2012, reflecting large investments in infrastructure, including those under the Sino-Congolese Cooperation Agreement.

20. Moreover, the government is committed to creating the fiscal space needed to meet its development and poverty reduction objectives. In particular, a large share of domestic revenue (40 percent) will be transferred to provinces, which will be responsible for the implementation of the education, health, and rural sector development. These large transfers will help strengthen human capital formation especially for vulnerable groups and the poor in line with our growth and poverty reduction strategy. Further, the country expects to reach the completion point under the HIPC Initiative in 2010 in order to benefit from debt relief associated with that initiative and debt cancellation under the MDRI. The government commits to conducting a prudent external debt management policy, including by relying on grants and highly concessional loans with a grant element significantly exceeding 35 percent for development financing. More importantly, the government will refrain from contracting or guaranteeing new debt that would jeopardize debt sustainability. It also intends to request from Paris Club creditors the extension over the medium term of the Cologne terms rescheduling agreement reached in 2002, the rescheduling of arrears accumulated since July 2006, and an exceptional rescheduling of debt service falling due in SeptemberDecember 2009. It is also planned to normalize relations with non-Paris Club bilateral and commercial creditors. In turn, it will avoid accumulating any new arrears on nonreschedulable external debt during the program period. The government will also seek to reach rescheduling agreements on similar terms with non-Paris Club creditors.

\footnotetext{
${ }^{9}$ Domestic fiscal balance is defined as total revenue (excluding grants and proceeds from signing bonus from the SCCA) minus total expenditure (excluding foreign financed projects and foreign interest payments due) plus the BCC's net operating deficit and net accumulation of domestic arrears).
} 


\section{Monetary and exchange policies}

21. The objective of monetary policy is price stability under a floating exchange rate regime. The $\mathrm{BCC}$ will strengthen the monetary policy framework by making its indirect monetary policy instruments more flexible, and enhancing its liquidity management and forecasting capacity. In the interest of transparency, it will adopt a communication strategy to signal its policies to markets. In addition, the BCC will apply an exchange rate policy aimed at smoothing exchange rate fluctuations. With a view to improving financial intermediation, boost confidence in credit institutions, and encourage de-dollarization, the BCC will also improve the quality of currency in circulation, strengthen banking supervision, and develop a payments and settlements system.

\section{Structural policies}

22. Structural reforms are focused on increasing domestic revenue, strengthening public financial management, restructuring and recapitalizing the $\mathrm{BCC}$, strengthening monetary policy and banking supervision, and putting the economy on strong and lasting growth path. The detailed measures and timetable are provided in the attached Matrix of Economic and Financial Policy Measures.

\section{Revenue and expenditure policy}

23. Tax policy (Matrix, I.A.): The government has adopted an action plan aimed at enhancing the efficiency of revenue collection while expanding the tax base. The main step will be the introduction of a single rate value-added tax (VAT), to be implemented by end2011. Other measures will be taken to rationalize tax exemptions and streamline the plethora of duties and taxes and their collection procedures. This effort will be complemented by the adoption of a new Customs Code in line with international best practices.

24. Customs administration (Matrix, I.B.): The government's is committed to put in place an efficient and modern customs administration. To this end, it will streamline customs procedures to reduce costs to the private sector while improving internal administration and controls to increase revenue collection. To simplify collection procedures, the government will expand one-stop customs windows to all important border posts, including in the eastern provinces. Moreover, it will transfer the customs activities currently performed by the Congolese Control Office (OCC) to the Custom and Excise Collection Agency (OFIDA), thereby making it the sole agency in charge of customs valuation and the collection of all taxes and fees. OCC's role will henceforth be limited to quality and standards control. OFIDA will enhance its information technology systems, based on SYDONIA++, in all customs offices. To improve oversight and ensure the satisfactory implementation of reforms, OFIDA will be converted into a general directorate of the Ministry of Finance.

25. Tax administration (Matrix, I.C.): The government will also establish an efficient and a modern tax administration. It will expand the network of corporate tax centers (CDIs) to manage the taxation of corporations at the national level, and establish tax centers (CIS) 
focused on middle and small enterprises. The development of a comprehensive taxpayer databank, combined with the adoption of a single identification numbers for all business entities, will improve post- and pre-collection controls and expand the tax base. The government will also strengthen post-collection controls by consolidating the audit and research functions of the tax department into a single office while improving its information system.

26. Collection of mining sector taxes (Matrix, I.D.): The government's objective is to increase the contribution of the mining sector to domestic revenue, which has been weak thus far. Accordingly, the government will strengthen the capacity of the tax department's (DGI) Large Taxpayers Office responsible for revenue collection from the sector, including by cooperating closely with specialized audit companies. Further, the collection of royalties and mining taxes (outside customs) will be transferred from the Directorate of Administrative and State Revenue (DGRAD) to the DGI, with the relevant legislation planned to be submitted to Parliament by April 2010. OFIDA will also establish specialized centers to assess adequately the quality and values of key mineral exports.

27. Public financial management (Matrix, I.E.): The government recognizes the need to improve public financial management to ensure the efficiency of budget preparation and implementation. It will implement over the program period an action plan built on the following five strategic pillars: (i) modernization of the legal framework, including the adoption of organic law on public finance and of a procurement code; (ii) improvement of the annual process of budget preparation, based on a medium-term fiscal framework that will be initially applied to key sector such as health and education; (iii) streamlining budget execution and cash management to improve the expenditure tracking process in order to avoid the accumulation of arrears; (iv) strengthening the reporting of expenditure execution through progressive computerization; and (v) reinforcing ex-ante and ex-post budget controls, including by the adoption of organic law on the audit office.

28. The Government intends to normalize its relationship with all its creditors. The government intends to start negotiate with all its creditors, in particular Paris Club creditors to obtain debt rescheduling in the most favorable terms given the weak repayment capacity of the country. Regarding the London Club, the government intends to finalize discussions for the cancellation of the commercial debt in the context of a buy-back program supported by the World Bank. It will seek to obtain from non-Paris Club creditors debt relief in terms that are at least comparable to those obtained from the Paris Club.

\section{The authorities are putting in place a comprehensive program to address the stock} of arrears and other claims on the government. An action plan for the gradual repayment of the stock of arrears at end-2007 has been put in place. In particular, fifty percent of the stock of arrears at end-2007 will be cleared in 2010 and the balance in 2011 . A repayment plan for the stock of 2008 arrears based the internal audit of the Inspection General des Finances will be designed no later than February 2010. In addition, the government will establish a plan to compile an inventory of all remaining claims on the government with proper auditing procedures. 


\section{Financial sector}

30. Central bank restructuring and recapitalization (Matrix, II.A.): The objective is to enhance the BCC's operationally capacity while putting it on a sound financial footing. Accordingly, the first step will be to restructure the BCC in line with the action plan developed with IMF technical assistance. Following the recent nominations of Director Generals and Directors, the BCC will put in motion the reorganization of departments and units within the central bank. It has adopted a three-year strategic plan that aims to improve governance and reinforce the bank's capacity to execute effectively its responsibilities. In parallel, the government will put in motion the process for recapitalizing the central bank, which is expected to take place in 2011.

31. Banking supervision (Matrix, II.C.): The government's objective is to strengthen banking supervision with a view of safeguarding financial system stability and soundness. Reforms in this area include improving the legal and regulatory framework, enhancing the operating skills of bank inspectors, introducing macro-prudential functions, and strengthening on-site and off-site inspection. Particular emphasis will be placed on increasing the minimum capital requirements for banks, adopting more stringent licensing procedures, and improving the quality and professionalism of inspectors.

\section{Other structural reforms}

32. Civil service reform (Matrix, III.A.): The improvement of administrative governance through capacity building is critical to national reconstruction. In August 2009, the government intends to approve a medium-term retirement strategy for public sector employees and to submit a draft civil service law to parliament in December 2009, including a regime governing provincial-level civil servants. The civil service census will be completed in December 2009 and that for the army and police will be completed by December 2010.

33. Business climate (Matrix, III.B.): An improved business climate is at the core of the government's strategy to revitalize the private sector. The government is determined to strengthen the protection of investments by joining the Organization for the Harmonization of Business Law in Africa (OHADA). It will also convert the National Investment Promotion Agency (ANAPI) into a one-stop shop for investment in the DRC. Planned changes in laws and regulations will help reduce red tape in establishing companies and settling commercial disputes. The government will continue to set up commercial and labor courts in the remaining provinces and adopt a commercial code aimed at eliminating tariff and non-tariff barriers, in compliance with the international agreements ratified by the DRC.

34. Decentralization (Matrix, III.C.): The government is placing particular emphasis on fiscal decentralization consistent with the 2006 Constitution. The Constitution establishes the principle of revenue sharing between the central government and the provinces. The goal is to improve the quality of public services at the local level. However, the decentralization process also poses certain risks, and the government has taken transitional measures to improve PFM at the provincial level in anticipation of the approval of the organic law on 
public finance. It will improve the mechanism for allocating national revenue to the provinces and decentralized territorial entities (ETDs) and to draft laws on the exclusive functions and powers of the provinces in order to guarantee effective public spending.

35. Debt management (Matrix, III.D.): The government will strengthen external and domestic debt management in order to limit risks of debt sustainability. To this end, the government acknowledges the exclusive authority of the Minister of Finance to make commitments on the government's behalf regarding borrowing by the central, provincial, and local authorities. External and domestic debt management will be centralized in the Public Debt Management Office (OGEDEP), which will produce a biannual statistical bulletin and quarterly forecasts of public debt service.

36. Economic governance (Matrix, III.E. \& F.): Transparency and improvement of economic governance are important elements of our strategy to encourage private investment. The government undertakes to publish, within 60 days, the partnership contracts between public and private enterprises, including the relevant financial and tax details. It will set up an independent anti-corruption agency. The government also intends to improve the national statistics in order to enhance the reliability of macroeconomic, social, and demographic data. Finally, the government will implement the action plan for the Extractives Industries Transparency Initiative (EITI) to bolster the transparency of the mining industries and their impact on tax revenue.

\section{Public enterprises (Matrix, III.G.): The government intends to accelerate public} enterprise reform to improve the delivery of services critical for promoting economic growth. It plans to divest from all enterprises involved in commercial activities. The process is ongoing and is implemented in two steps. In the first step, the government has initiated the privatization of the management of public enterprises, which is aimed at stabilizing and improving their financial situation. In the second step, the government will develop restructuring and privatization plan. To facilitate the public enterprise restructuring and privatization process, the government will develop social programs to help redundant public enterprise employees eligible to retirement or departing on a voluntary basis and a plan to address cross-debts between public enterprises and between public enterprises and the government. Enterprises that are involved in nonprofit activities or that are extensions of government agencies will be transformed, as appropriate, into public establishments while those whose activities have ceased or are no longer warranted will be dissolved.

\section{Policy FOR THE ReST OF 2009 AND FOR 2010}

38. The macroeconomic objectives for $\mathbf{2 0 0 9}$ are: (i) a real GDP growth rate of 2.7 percent sustained by the partial revitalization of the mining and construction sectors and by the expansion of the agriculture, telecommunications, and transport sectors; (ii) limiting year-onyear inflation at 48.7 percent; and (iii) maintain the coverage of gross foreign reserves at about 10 weeks of nonaid imports. To attain these objectives, prudent macroeconomic policies will need to be put in place, as well as structural and sectoral reforms to promote a business climate conducive to the development of a viable private sector that would underpin strong and lasting economic growth. 
39. The objective of fiscal policy for the rest of 2009 is to limit the government's recourse to domestic financing. In this regard, the domestic fiscal deficit (cash basis) will be limited to 3.3 percent of GDP, consistent with a reduction of net credit to the government of 1.2 percent of GDP. Tax revenue for the year as a whole is estimated at 15.6 percent of GDP and domestically financed expenditure will be capped at 17.5 percent of GDP. Foreignfinanced investment is projected to reach 6.2 percent of GDP, including infrastructure projects implemented under the Sino-Congolese Cooperation Agreement.

\section{The excessive level of expenditure commitments in the pipeline at end-October} (CF 258 billion) requires corrective measures to ensure consistency with the treasury plan 2009. Given the level of commitments at end-October, certain sectoral ministries and other institutions have very little room to maneuver. To address the situation, the government has (i) cancelled all commitments for which goods and services have not been committed (CF 68 billion); (ii) postponed the repayment of part of the arrears accumulated in 2007-08 (CF 31 billion), pending their audit; and (iii) stopped all new commitments at end-October. These measures should allow achieve fiscal policy objectives.

41. The objective of monetary policy in $\mathbf{2 0 0 9}$ is to contain inflation in the context of a floating exchange rate regime. The expansion of the monetary base will be limited to 19.6 percent in 2009. Broad money is projected to grow at 33.8 percent. To achieve the monetary targets, the BCC will continue to sell BTRs (central bank bills) to mop up excess liquidity in the economy. Its plan for selling BTRs will be drawn up in close collaboration with the Treasury. Exchange rate intervention will aim at smoothing undue volatility while achieving the reserve target. Subject to the reserve target, the central bank also could sell foreign exchange to help mop up excess liquidity, especially that generated by government use of external budget support.

42. Key structural reforms for the period July 2009 to June 2010 include the promulgation of a new organic public finance law, adoption of a world class procurement code, streamlining of duties and taxes, evaluation and elimination of ad hoc tax exemptions, the transfer of OFIDA into a department of the Ministry of Finance, the restructuring of the $\mathrm{BCC}$ and the modernization of its monetary policy instruments, and the submission to Parliament of a new statistics law.

43. The objective of fiscal policy for $\mathbf{2 0 1 0}$ will be to limit recourse to domestic financing. In this context, the domestic fiscal deficit (cash basis) will be limited to 1.4 percent of GDP, consistent with zero net credit to the government from the banking system. Tax revenue and domestically financed expenditure are both projected to be 17.9 percent of GDP and 18.8 respectively. Foreign-financed capital expenditure is projected at 14 percent of GDP.

44. The main objective of monetary policy in $\mathbf{2 0 1 0}$ will be to further reduce inflation. In that context, the growth of base money is targeted at 22 percent in 2010 . With money multiplier expected to remain stable, broad money growth is also projected to grow in line with base money. The BCC will review its policy rate taking into account progress toward the inflation objectives. 


\section{Technical Assistance Needs and Priorities for Training}

45. The government will request technical assistance from the international community in macroeconomic and structural reforms. It is grateful for the technical assistance provided by the IMF through its Central African Regional Technical Assistance Centers (Central AFRITAC), the World Bank, the AfDB, the UNDP, and other development partners. This technical assistance will be a determining factor in the success of the decentralization process and in the formulation and implementation of sectoral policies as defined in our PRGS. The government will prepare a list of technical assistance needs and priorities for strengthening institutional capacity at the provincial level.

\section{Program Monitoring}

\section{The monitoring of program will be carried out on the basis of quantitative} performance criteria and benchmarks as well as structural benchmarks in the context of semi-annual reviews (Tables 1-2). The first review will be completed by end-June 2010 and will cover macroeconomic developments and policies for 2010 and progress in structural reforms in tax and customs administration, PFM, and financial sector reform. An update safeguards assessment is currently in progress in the context of the proposed PRGF arrangement, as required by the IMF safeguards policy.

\section{In the context of the proposed PRGF, an update safeguards assessment of the} Banque Centrale du Congo (BCC) is currently in progress. The previous update assessment, concluded in January 2008, found a strengthened external audit mechanism, but significant safeguards risks remained in the control framework of the central bank, including serious concerns regarding the bank's independence. As a result, the assessment recommended specific measures for implementation in advance of a Board discussion of a new PRGF arrangement. The BCC agreed with the findings of the assessment and committed to implementing the safeguards recommendations, some of which were prior actions and others are structural benchmarks in the proposed PRGF program. Staff is monitoring BCC's implementation of the measures. The update assessment will be completed no later than the first review.

48. The government is strengthening its program monitoring and evaluation capacity. A National Steering Committee on the Poverty Reduction Strategy (CNP-SRP) will be established directly under the authority of the Prime Minister. This Committee will be assisted by the Interministerial Technical Committee in the Prime Minister's Office and will supervise the activities of the two interministerial committees: one in charge of drafting the National Poverty Reduction Strategy (CI-SNLCP) and the other in charge of steering and monitoring the implementation of the PRS (CISPI). The CI-SNLCP will be chaired by the Minister of Planning, assisted by the other members, namely the ministers in charge of priority sectors. Its mission will be to steer the process of preparing the national poverty reduction strategy. As part of its mission, it will work closely with the Steering Committee for the Poverty Reduction Strategy Preparation Process (UPPE-SRP). The CISPI will be chaired by the Minister of Finance and its members will include the ministers of Budget, 
Economy, Planning, Trade, and those ministers concerned with the implementation of the reforms. It will be assisted by the technical committee for monitoring reforms (CTR). The government has also established an Interministerial Technical Committee (CTSP) that monitors the implementation of the government's macroeconomic program supported by the PRGF arrangement. 
Table I.1. Democratic Republic of the Congo: Quarterly Quantitative Performance Criteria and Indicative Targets, 2009-10

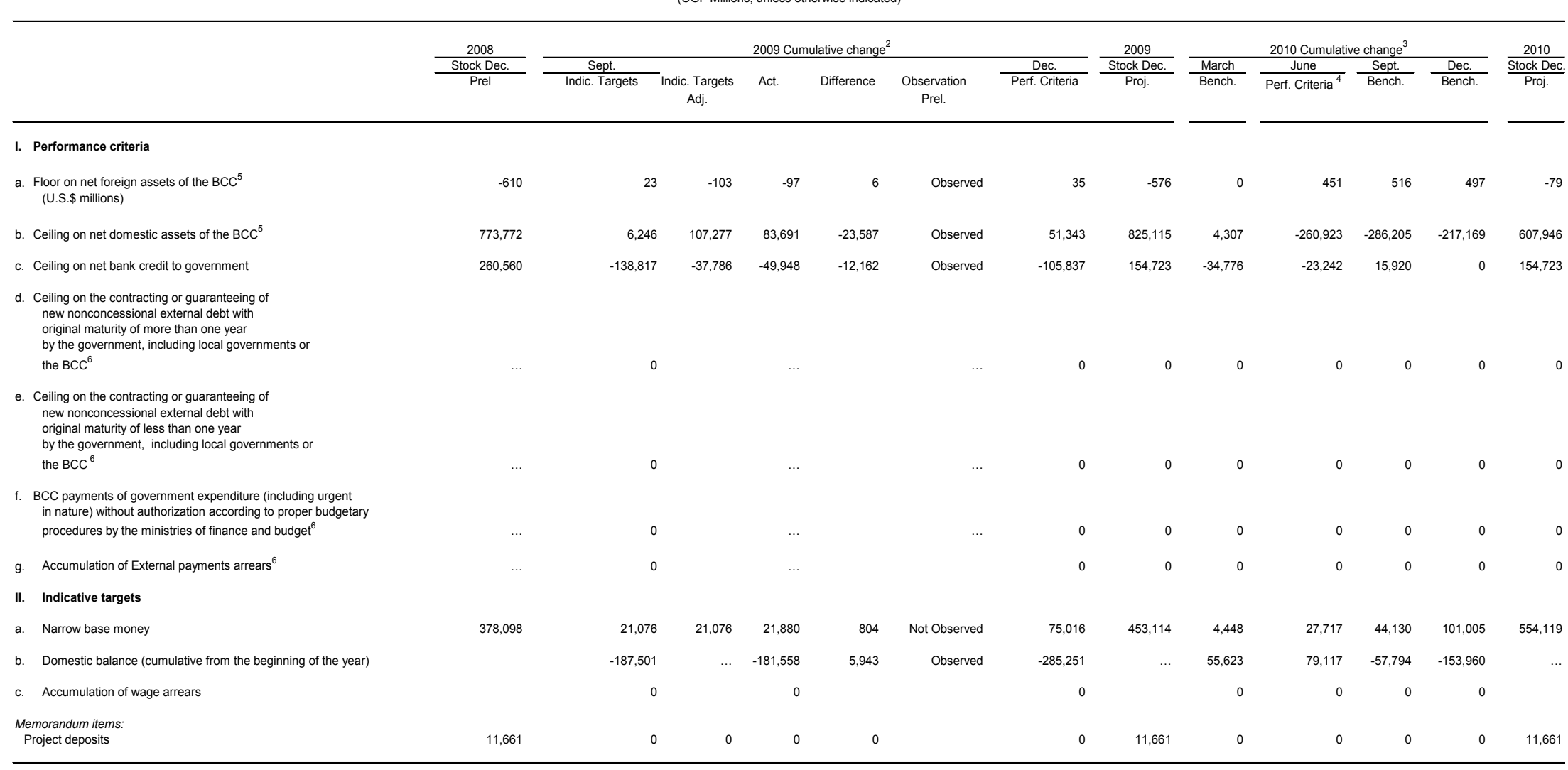

Source: Congolese authorities; and IMF staff estimates and projections.

${ }^{1}$ For definition and adjustors see the attached Program Monitoring Section of Memorandum of Economic and Financial Polices and the Technical Memorandum of Understanding. ${ }^{2}$ Cumulative changes are calculated from end-December 2008.
${ }_{3}^{3}$ Cumulative changes are calculated from end-December 2009 .

These performance criteria may be revised in the context of the first review of the PRGF arrangement.

${ }^{5}$ The stocks of net foreign assets and net domestic assets of the BCC are valued at the program exchange rates (US\$1 = CGF 639.32; and 1 Euro = 905.07)

${ }^{6}$ These performance criteria will be monitored on a continuous basis. 
Table I.2. The Democratic Republic of the Congo: Structural Conditionality and Macroeconomic Relevance, 2009-10

\begin{tabular}{|c|c|c|}
\hline Measure & Status & Macroeconomic Rationale \\
\hline \multicolumn{3}{|l|}{ Prior Actions } \\
\hline $\begin{array}{l}\text { Establishment of a committee to assess tax incentives and exemptions granted } \\
\text { under the mining code, investment code, and special conventions in } \\
\text { accordance with terms of reference devised in consultation with IMF staff. }\end{array}$ & Observed & $\begin{array}{l}\text { Enhance revenue mobilization to achieve the fiscal objectives of the } \\
\text { program }\end{array}$ \\
\hline $\begin{array}{l}\text { Collection of all revenue from the mining sector by a new unit specialized in } \\
\text { mining taxation along with the Large Taxpayers Unit. }\end{array}$ & Observed & $\begin{array}{l}\text { Enhance revenue mobilization to achieve the fiscal objectives of the } \\
\text { program. }\end{array}$ \\
\hline $\begin{array}{l}\text { Finalization of the audit of domestic arrears accumulated as of end-December } \\
2007 \text { and adoption by government of a plan of eliminating them. }\end{array}$ & Observed & $\begin{array}{l}\text { To ensure meeting the obligations of the government to the private } \\
\text { sector and avoid stunting private sector development and economic } \\
\text { growth. }\end{array}$ \\
\hline $\begin{array}{l}\text { Operational implementation of the National Financial Intelligence Unit } \\
\text { (CENAREF) in the context of anti-money laundering efforts. }\end{array}$ & Observed & Promote orderly financial sector development \\
\hline $\begin{array}{l}\text { Amend the Sino-Congolese cooperation agreement to ensure that its terms are } \\
\text { consistent with debt sustainability. }\end{array}$ & Observed & Strengthen debt management to achieve debt sustainability. \\
\hline $\begin{array}{l}\text { Selection of an audit firm of international repute to audit the PRGF criteria } \\
\text { for the duration of the program }(2009-11)\end{array}$ & Observed & Reinforce program monitoring and control. \\
\hline $\begin{array}{l}\text { Appointment of directors at the } \mathrm{BCC} \text {, to be in charge of the new Directorate } \\
\text { created in the context of the central bank restructuring }\end{array}$ & Observed & $\begin{array}{l}\text { Promote the modernization of the } \mathrm{BCC} \text {, in particular in improve its } \\
\text { monetary policy design and banking supervision }\end{array}$ \\
\hline \multicolumn{3}{|l|}{ Structural benchmarks } \\
\hline Restructuring one large commercial bank. & End-Dec. 2009 & Systemic risk. \\
\hline $\begin{array}{l}\text { Adoption by the Council of Ministers of an action plan prepared by the } \\
\text { committee responsible for assessing tax incentives and exemptions granted } \\
\text { under the mining code, investment code, and special convention. }\end{array}$ & End- Dec. 2009 & $\begin{array}{l}\text { Enhance revenue mobilization to achieve the fiscal objectives of the } \\
\text { program. }\end{array}$ \\
\hline Implementation of ASYCUDA++ in the customs offices in Kinshasa & End- Dec. 2009 & $\begin{array}{l}\text { Enhance revenue mobilization to achieve the fiscal objectives of the } \\
\text { program. }\end{array}$ \\
\hline $\begin{array}{l}\text { Repayment plan for } 2008 \text { arrears based the internal audit of the Inspection } \\
\text { General des Finances and establish a plan to identify and audit of any } \\
\text { remaining government arrears. }\end{array}$ & End- Feb. 2010 & $\begin{array}{l}\text { To ensure meeting the obligations of the government to the private } \\
\text { sector and avoid stunting private sector development and economic } \\
\text { growth. }\end{array}$ \\
\hline $\begin{array}{l}\text { Submission to Parliament of a draft organic law on public finance that inter } \\
\text { aliea prohibits provinces from borrowing from commercial banks and the } \\
\text { Central Bank. }\end{array}$ & End-March. 2010 & Establish fiscal discipline based on transparent rules and regulations \\
\hline $\begin{array}{l}\text { Generation and publishing of a monthly budget execution tables (reconciled } \\
\text { with the TOFE, the BCC, and the monetary statistics), no later than } 30 \text { days } \\
\text { after the end of each month. }\end{array}$ & Continuous & Strengthen the monitoring of the program \\
\hline $\begin{array}{l}\text { Inclusion of externally financed expenditure in the monthly budget execution } \\
\text { tables (reconciled with the TOFE, the BCC, and the monetary statistics), no } \\
\text { later than } 3 \text { months after the end of each month. }\end{array}$ & Continuous & Strengthen the monitoring of the program \\
\hline $\begin{array}{l}\text { Publication of mining sector partnership contracts between public and private } \\
\text { enterprises with } 60 \text { days of signature (including urgent expenditure payments) } \\
\text { without prior authorization by the Minister of Finance in accordance with } \\
\text { existing legislation. }\end{array}$ & Continuous & $\begin{array}{l}\text { Enhance economic governance and ensure accountability for public } \\
\text { resources }\end{array}$ \\
\hline $\begin{array}{l}\text { No payment by the BCC of expenditures on behalf of the government } \\
\text { (including urgent expenditure payments) without prior authorization by the } \\
\text { Minister of Finance in accordance with existing legislation. }\end{array}$ & Continuous & $\begin{array}{l}\text { Enhance economic governance and ensure accountability for public } \\
\text { resources }\end{array}$ \\
\hline
\end{tabular}

Source: Congolese authorities; and IMF staff assessments 
Table I.3. Democratic Republic of the Congo: Matrix of Economic and Financial Policy Measures for 2009-12

\begin{tabular}{lll}
\hline Area & Measures & Timing \\
\hline
\end{tabular}

\section{Tax and fiscal policies and reforms}

\section{A. Tax policy}

\section{Objective: Modernize the Tax System}

1. Reduce the number of low-yielding taxes:

- Adoption by the Council of Ministers the report on identifying lowyielding taxes.

- Submit to Parliament draft laws on the elimination of these taxes.

End-December 2009

End-June 2010

2. Limit tax and customs incentives and exemptions:

- Adoption by the Council of Ministers of the action plan prepared by the Commission in charge of evaluation of incentives and fiscal exemptions granted in the framework of the Mining Code, Investment Code, and special conventions. (Structural benchmark)

- Refrain from granting or renewing discretionary tax incentives and exemptions outside the investment, mining, and forestry codes.

3. Reduce the number of nuisance taxes and border fees:

- $\quad$ Produce a report identifying parafiscal taxes and border fees, without a quid pro quo, and adopt an action plan to eliminate them

- Implement this action plan.

4. Establish the VAT:

- Adopt and enact the draft law establishing the modern single-rate VAT.

- Collect the first VAT tax returns from businesses by the DGI.

5. Implement the new customs code:

- Adoption by the Council of Ministers.

- Submission to the Parliament.

- $\quad$ Promulgation by the President.

Objective: Modernize Customs Administration

B. Customs administration
1. Transfer customs activities from the Congolese Office of Control (OCC) to OFIDA:

- Adoption by the Council of Ministers of the new statutes for OCC and OFIDA.

- Approval by the Council of Ministers the action plan for the effective transfer of customs activities from the OCC to OFIDA.

2. Issuance by the Prime Minister of a decree to convert OFIDA into a Directorate-General of Customs reporting to the Ministry of Finance.

3. Strengthen the rules requiring pre-inspection of imports by BIVAC; for goods not yet subject to pre-inspection, OFIDA will determine their value by using the BIVAC database.
End-December 2009

End-December 2010

End-December 2010

End-January 2012

End-December 2009

Ongoing

End-December 2009

End-December 2009

End-June 2010

December 2009

April 2010

End-December 2009

End-December 2009 
Table I.3. Democratic Republic of the Congo: Matrix of Economic and Financial Policy Measures for 2009-12 (Continued)

\begin{tabular}{|c|c|c|}
\hline Area & & Measures \\
\hline \multirow{5}{*}{$\begin{array}{l}\text { B. Customs } \\
\text { administration } \\
\text { (continued) }\end{array}$} & & Objective: Modernize Customs Administration \\
\hline & 4. & $\begin{array}{l}\text { Implement ASYCUDA++ in all customs offices where the volume and type of } \\
\text { trade justify it: }\end{array}$ \\
\hline & & - $\quad$ At Kinshasa. (Structural benchmark) \\
\hline & & $\begin{array}{l}\text { - At other customs office that account for either a minimum of } 5 \text { percent } \\
\text { of imports (CIF value) or a minimum of } 10 \text { percent of transit (CIF } \\
\text { value). }\end{array}$ \\
\hline & 5. & Introduce the one-stop window at all customs offices. \\
\hline \multirow{9}{*}{$\begin{array}{l}\text { C. Tax } \\
\text { administration }\end{array}$} & & Objective: Strengthen Tax Administration \\
\hline & 1. & Merge the DGRAD with the DGI: \\
\hline & & $\begin{array}{l}\text { - Set up an independent commission to study the advisability and } \\
\text { feasibility of merging the DGRAD with the DGI. }\end{array}$ \\
\hline & & $\begin{array}{l}\text { - Adoption by the Council of Ministers the report on the advisability and } \\
\text { feasibility of merging the DGRAD with the DGI. }\end{array}$ \\
\hline & 2. & $\begin{array}{l}\text { Extend the use of the new single tax identification number (NIF) to all } \\
\text { provinces. }\end{array}$ \\
\hline & 3. & Transfer all enterprises that meet the eligibility criteria to the DGE portfolio. \\
\hline & 4. & $\begin{array}{l}\text { Establish tax centers (CDIs) in all provinces to manage medium-sized } \\
\text { enterprises: }\end{array}$ \\
\hline & & - $\quad$ Establish three CDIs: Bas Congo, Nord Kivu and Sud Kivu. \\
\hline & & - Establish CDIs in all other provinces. \\
\hline
\end{tabular}

End-December 2009

End-December 2011

End-December 2011

End-September 2009

End-March 2010

End-June 2010

End-December 2009

End-March 2010

End-December 2011

\section{Objective: Increase Revenues from the Mining Sector}

1. Sign a memorandum of understanding with other public institutions on the exchange of information on mining sector exports.

2. Strengthen tax administration in the mining sector:

- Strengthen the capacity of the specialized unit, including by means of agreements with specialized audit companies and international consulting firms.

- Submit to Parliament draft legislation to transfer all responsibility for tax administration in the mining sector from the DGRAD to the new specialized unit in the DGE, which becomes responsible for internal assessment, in line with international best practice, for the audit and collection of all taxes, fees, and royalties from the major mining companies.

3. Starting in July 2009, conduct annual audits by the Inspectorate General of Finance, six months after the year in question, to verify that all tax revenue paid by the mining companies have been transferred to the general account of the Treasury.

4. In the context of strengthening the capacity of OFIDA, establish centers of expertise for the principal mining exports at Kasumbalesa and Sakania in Katanga.
End-December 2009

End-December 2009

End-October 2010

Ongoing

End-June 2010 
Table I.3. Democratic Republic of the Congo: Matrix of Economic and Financial Policy Measures for 2009-12 (Continued)

Area
E. Public
finance
management
(i) Legal and
institutional
aspects

(ii) Preparation of
the budget

Measures

Timing

Objective: Strengthen Public Finance Management

\section{Adoption by the Government of a Public Financial Management Action Plan}

End-March 2010

1. Submit to Parliament a draft organic law on public finance that specifically prohibits provinces from borrowing from commercial banks and the Central Bank. (Structural Benchmark)

2. Submit to Parliament the 2008 draft budget review law by the Audit Office.

End-December 2009

3. Adopt the new Public Procurement Code and its implementing regulations.

End-December 2009

4. Reform the financing of the tax collection agencies:

- Eliminate the transfers to the three government tax collection agencies.

End-July 2010

- Replace performance premiums with operating and capital budget

End-January 2011 appropriations and by incentives for agents in the tax collection agencies.

5. Approve general regulations on government accounting.

End-December 2010

6. Submit to Parliament a draft organic law on the Audit Office.

End-December 2010

7. Review the regulatory framework of the Inspectorate General of Finance.

End-December 2010

1. Develop a medium-term budget framework:

- For the ministries of health and education.

End-March 2010

- In all ministries, in close cooperation with the sector ministries.

End-December 2012

(iii) Budget execution

1. Publish a decree by the Minister of Finance to oblige the government to pay resident suppliers in the DRC in local currency.

2. Conduct an audit of all accounts of government entities at the BCC and at commercial banks.

3. Strengthen the computerized expenditure process by improving the software and updating the hardware.

End-December 2009

End-December 2009

End-December 2010

4. Regularization of arrears on domestic payments:

- Prepare repayment plan for the stock of domestic payment arrears at end2008 based on audit of the Inspection General des Finance. (Structural Benchmark)

- Implement action plan for gradual repayment of stock of arrears at end2008.

5. Reduce the use of the exceptional expenditure procedure by:

- Strictly adhere to the December 2008 Decree and stopping the use of "mise à dispositions".

- Prepare an exit strategy to integrate the urgent spending procedure into the computerized expenditure cycle

6 Prepare a commitment plan that is consistent with the cash-flow plan.

(iv) Accounting system and budget reports
1. Finalize the implementation of double entry bookkeeping at the Directorate of the Treasury in the Ministry of Finance and expand it to all revenue collection agencies.

2. Production and publication of monthly tables: (Structural benchmark)

- Generate and publish monthly budget execution tables (reconciled with the TOFE, the BCC, and the monetary statistics), no later than 30 days after the end of each month.

- Include externally financed expenditures in the monthly budget execution tables ((reconciled with the TOFE, the BCC, and the monetary statistics), no later than 3 months after the end of each month.
February 2010

2010-11

Ongoing

End-December 2009

Ongoing

End-December 2009

Ongoing 
Table I.3. Democratic Republic of the Congo: Matrix of Economic and Financial Policy Measures for 2009-12 (Continued)

\begin{tabular}{|c|c|c|}
\hline Area & & Measures \\
\hline $\begin{array}{l}\text { E. Public } \\
\text { finance } \\
\text { management } \\
\text { (concluded) }\end{array}$ & & Objective: Strengthen Public Finance Management \\
\hline $\begin{array}{l}\text { (v) Payroll } \\
\text { management }\end{array}$ & 1. & $\begin{array}{l}\text { Check consistency between human resource database and payroll management } \\
\text { for Extend the Simplified Transitional System (PTS) for the payroll to: } \\
\text { - All public officials and government employees. } \\
\text { - All provinces. }\end{array}$ \\
\hline & 2. & $\begin{array}{l}\text { Unify the various government employee management systems, particularly by } \\
\text { revising the structure of schedules applicable to those systems. } \\
\text { II. Monetary and Financial Policy }\end{array}$ \\
\hline A. BCC Reform & & Objective: Make the Central Bank Independent and Efficient \\
\hline $\begin{array}{l}\text { (i) Restructuring } \\
\text { of the } B C C\end{array}$ & 1. & $\begin{array}{l}\text { Implement the new organization and restructuring of the BCC. } \\
\text { Establish organic units under every directorate in accordance with the } \\
\text { organizational chart developed with the technical assistance of the IMF } \\
\text { MCM department. } \\
\text { Approve the delegation of responsibilities to General Directors and } \\
\text { Directors for all ordinary transactions of the central bank and all other } \\
\text { administrative decisions developed with the technical assistance of the } \\
\text { IMF MCM department. }\end{array}$ \\
\hline
\end{tabular}

(ii) Recapitalization of the $B C C$

\section{B. Monetary policy \\ (i) Liquidity forecasting/money market interventions \\ (ii) Currency issuance and quality of currency in circulation}

(iii) Exchange transactions
1. Adoption by the Council of Ministers of the action plan to recapitalize the BCC based on the recommendations of the IMF technical assistance mission.

2. Submit to the Parliament the draft law on the BCC recapitalization.

3. Adoption by the Council of Ministers of the budget required to recapitalize the central bank by issuing government securities, at interest rates and maturities to be determined in line with market conditions.

4. Recapitalize the BCC.

End-March 2010

End-December 2010

End-December 2010

End-September 2009

End-September 2009

End-February 2010

End-October 2010

End-November 2009

End-June 2011

Objective: Improve the BCC's Capacity to make Monetary and Exchange Policy

1. Publicize the instruction authorizing the trading of BTRs on a secondary market.

End-September 2009

$2009-11$

1. Increase the banknote processing capacity to 100 percent by allocating the necessary resources to the Treasury Directorate:

- 40 percent by June 2010.

- 70 percent by end-December 2010 .

- 100 percent by June 2011 .

2. Adopt a 3-year strategy to reform the currency issuance function in the provinces, to improve the quality of currency in circulation, in coordination with assistance from the IMF expert.

3. Issuance of coins in 2010 in accordance with the strategy developed with the assistance of the IMF expert.

1. Hold regular foreign exchange auctions and publish an advance notice on the Internet, to ensure a regular presence and transparency on the foreign exchange market.
End-October 2009

End-September 2010

Continuous 
Table I.3. Democratic Republic of the Congo: Matrix of Economic and Financial Policy Measures for 2009-12 (Continued)

\begin{tabular}{|c|c|c|}
\hline Area & & Measures \\
\hline $\begin{array}{l}\text { C. Supervision/ } \\
\text { Banking system }\end{array}$ & & $\begin{array}{l}\text { Objective: Enhance Banking Supervision Capacity and the Health of the } \\
\text { Banking System }\end{array}$ \\
\hline \multirow[t]{2}{*}{ (i) Supervision } & 1. & $\begin{array}{l}\text { Strengthen the central bank's supervision capacity by implementing new } \\
\text { commercial bank prudential ratios. }\end{array}$ \\
\hline & 2. & $\begin{array}{l}\text { Implement a new matrix of sanctions for noncompliance with the banking } \\
\text { supervision regulations. }\end{array}$ \\
\hline \multirow[t]{3}{*}{$\begin{array}{l}\text { (ii) Banking } \\
\text { system }\end{array}$} & 1. & $\begin{array}{l}\text { Complete the audits to assess the quality of the loan portfolio: } \\
\text { - Of three banks, and establish a plan to restructure and/or recapitalize } \\
\text { them, in coordination with their boards of directors. } \\
\text { - Of all remaining banks, and establish a plan to restructure and/or } \\
\text { recapitalize them. }\end{array}$ \\
\hline & 2. & $\begin{array}{l}\text { Adopt a strategy to strengthen the health of commercial banks based on the } \\
\text { results of the March } 2009 \text { evaluation study undertaken with technical assistance } \\
\text { from IMF and World Bank experts. }\end{array}$ \\
\hline & 3. & Restructuring one large commercial bank \\
\hline $\begin{array}{l}\text { D. Accounting } \\
\text { and transparency }\end{array}$ & & Objective: Improve Accounting and Transparency \\
\hline \multirow[t]{2}{*}{$\begin{array}{l}\text { (i) Accounting and } \\
\text { audit operations }\end{array}$} & 1. & $\begin{array}{l}\text { No payments on government expenditures (including urgent expenditure) by the } \\
\text { BCC that have not been authorized in advance by the Minister of Finance, in } \\
\text { accordance with existing legislation. (Structural benchmark) }\end{array}$ \\
\hline & 2. & $\begin{array}{l}\text { Approval by the BCC's board of directors an action plan to apply the } \\
\text { international accounting standards (IFRS). }\end{array}$ \\
\hline $\begin{array}{l}\text { (ii) Transparency } \\
\text { and communication }\end{array}$ & 1. & $\begin{array}{l}\text { Publish the financial statements and audit reports of the BCC, no later than } 6 \\
\text { months after the end of the year, including the auditor's opinion. }\end{array}$ \\
\hline
\end{tabular}

Timing

End-October 2009

End-December 2009

End-December 2009

End-March 2010

End-November 2009

End-December 2009

\section{Other Structural Reforms}

A. Civil Service

B. Business climate

C.

\section{Decentralization}

Policy

Tax and fee classifications for provinces and ETDs
1. Complete the survey of the Civil Service.

2. Complete the survey of the Army and Police.

1. Submit to Parliament the OHADA membership treaty.

2. Eliminate all tariff and nontariff barriers that are not consistent with the international agreements ratified by the DRC.

3. Submit to Parliament the draft law on liberalization of the insurance sector.

4. Set up commercial courts in:

- Kisangani and Matadi.

- The remaining provinces.

1. Implement the 40-percent revenue transfers formula:

- Transitional period.

- Implementation.

1. Submit to the Parliament a draft law on the classification of taxes and fees of the provinces and ETDs.
End-April 2010

End-December 2010

End-September 2009

End-March 2010

End-March 2010

End-December 2010

End-December 2011

2009

January 2010

End-October 2010 
Table I.3. Democratic Republic of the Congo: Matrix of Economic and Financial Policy Measures for 2009-12 (Concluded)

\begin{tabular}{l} 
Area \\
\hline C. Decentralization \\
(concluded)
\end{tabular}

Strengthen PFM at the provincial level

\section{Debt management}

\section{F. Statistics}

\section{G. Public enterprise reform}

\section{E. Transparency}

1. Simplify budget classification at the provincial level:

- Revise the revenue and expenditure classifications.

End-September 2010

- Introduce a simplified classification within the framework of the 2012 Budget Law for the provinces.

2. Develop and approve an action plan to strengthen public financial management (PFM) capacity at the provincial level.

3. Introduce a computerized expenditure process in all provinces and link it to the central government's expenditure process.

1. Execute the action plan for effective implementation of the decree that centralizes external debt management in the OGEDEP including all information concerning public debt as well as all application measures.

2. Effectively collect and centralize data on internal and external public debt in the OGEDEP.

1. Publication within 60 days:

- Of partnership agreements between public and private enterprises (including information on bonus signing shares, taxation system, private shareholders, and members of the board of directors. (Structural benchmark)

- Of the negotiation results between mining companies and the Timing

End-December 2011

End-September 2010

End-December 2010

End-December 2009

End-December 2009 government regarding the review of mining contracts.

2. Implement the Extractive Industries Transparency Initiative.

End-March 2010

3. Establish an independent anti-corruption agency.

End-June 2010

1. Submit to Parliament by the government the draft statistics law.

End-December 2009

2. Revision of national accounts statistics:

- $\quad$ Finalize national accounts estimates (SNA93) for 2006-07.

End-March 2010

- Adoption by the government of national accounts in accordance with SNA93.

3. Finalization and adoption by the government the national statistical development strategy (SNDS).

1. Develop and submit to the Council of Ministers the social programs for the target enterprises (ONATRA, RVA, SNCC, REGIDESO, and SNEL)

2. Evaluate cross-debts between the government and the public enterprises, and between public enterprises:

- Recruit the Cabinet.

End-March 2010

- Adopt the action plan by the government. 


\section{APPENDiX I \\ ATTACHMENT II \\ TRANSLATED FROM FRENCH \\ DEMOCRATIC REPUblic OF THE CONGO \\ TECHNICAL MEMORANDUM OF UNDERSTANDING \\ ON PROGRAM IMPLEMENTATION}

Kinshasa, November 30, 2009

1. This memorandum defines the quantitative targets that will be used to assess the performance by the Democratic Republic of the Congo under the program supported by the Poverty Reduction and Growth Facility (PRGF). It also specifies the content and frequency of the data needed for program monitoring. Unless otherwise indicated, all the quantitative targets are measured in terms of cumulative changes since January 1, 2009. Variables denominated in U.S. dollars will be converted to Congo francs by using the program exchange rate of CF 639.32 per US\$. Variables denominated in currencies other than the U.S. dollar (excluding SDRs and euros) will first be converted to U.S. dollars at the endperiod US\$/currency exchange rate. Variables denominated in SDR will be valued at the program exchange rate of CF 994.02 per SDR. Variables denominated in euros will be valued at the program exchange rate of CF 905.07 per Euro.

2. Institutional coverage: The central government comprises all units of government that exercise authority over the entire economic territory. However, unless otherwise indicated for the purposes of this memorandum, the central government does not include nonprofit organizations controlled and financed by the central government. The banking system is understood to mean the Central Bank of the Congo (BCC) as well as existing or newly licensed commercial banks.

\section{Quantitative Performance Criteria}

3. Quantitative performance criteria were established for end-December 2009 and end-June 2010, and indicative targets were set for end-September 2009 and end-March 2010 with regard to the following variables:

- $\quad$ Changes in the net foreign assets of the BCC;

- $\quad$ Changes in the net domestic assets of the BCC;

- Changes in net banking system credit to the government (central government);

- $\quad$ Payments of government expenditures (including emergency expenditures) by the BCC without the prior authorization according to proper budgetary procedures by the Ministries of Budget and Finance; 
- Nonconcessional medium- and long-term foreign loans contracted or guaranteed by the central government, local governments, or the $\mathrm{BCC}$;

- Nonconcessional short-term foreign loans contracted or guaranteed by the State, local governments, or the $\mathrm{BCC}$; and

- The accumulation of external payment arrears.

\section{A. Floors on the net foreign assets (NFA) of the BCC}

4. Definition: NFA are defined as the difference between the BCC gross international reserves and its total liabilities. Gross foreign assets are defined as the sum of the following items: (i) monetary gold holdings of the BCC; (ii) SDR holdings; (iii) convertible claims on nonresidents, such as foreign deposits and foreign securities. The following items are excluded from the definition of gross reserves: claims on residents in foreign exchange, nonconvertible currency holdings, and reserves that are encumbered or pledged in one form or another, including but not limited to reserve assets used as collateral or security for foreign third-party liabilities. Foreign liabilities are all BCC foreign exchange liabilities to nonresidents (including SDR allocations), including the IMF.

5. The following adjustments will be made to the NFA floors.

- Balance of payments support (BPS): NFA will be adjusted (i) upward by an amount equivalent to total BPS in excess of the programmed levels, (ii) in July-December 2009 downward by an amount equivalent to the lesser of total shortfalls of BPS (excluding that from the IMF) relative to programmed levels and US\$45 million; (iii) downward by an amount equivalent to any shortfall in debt relief under the MDRI from the IMF; and (iv) in January-December 2010 downward by an amount equivalent to the excess of external debt service payments relative to programmed amounts.

- Signing bonus from the Sino-Congolese Cooperation Agreement (SCCA): NFA will be adjusted (i) upward by an amount equivalent to total disbursement of signing bonus from SCCA in excess of the programmed levels; (ii) downward by an amount equivalent to total shortfalls in the disbursement of signing bonus from SCCA relative to programmed levels.

- Privatization proceeds in convertible currencies (PPCC): NFA will be adjusted (i) upward by an amount equivalent to total PPCC in excess of the programmed levels; and (ii) in July-December 2009 downward by an amount equivalent to the lesser of total shortfalls in PPCC relative to programmed levels and US\$45 million. 
6. Definition: BPS includes (all grants and loans) minus (grants and loans for externally financed projects) plus (debt relief granted by the IMF under the Multilateral Debt Relief Initiative) minus (external debt service payments (see $\uparrow 7$ below)). External financing for the National Disarmament, Demobilization, and Reintegration Program (DDR) falls under the heading of externally financed projects and is therefore not included in the definition of BPS.

7. Definition: scheduled external debt service payments (excluding those to the IMF) are defined as debt service due (principal and interest) minus debt relief (excluding debt relief offered by the IMF).

\section{B. Ceilings on the net domestic assets (NDA) of the BCC}

8. Definition: The NDA of the BCC are defined as base money (see paragraph 18 below) minus NFA. Based on this definition, the NDA of the BCC include: (i) net credit to the government (central government) (see paragraph 10 below); (ii) credit to the private sector; (iii) credit to parastatal enterprises; (iv) credit to commercial banks; and (v) other net assets.

9. The following adjustments will be made to the NDA ceilings.

- BPS: NDA will be adjusted (i) downward by an amount equivalent to total BPS in excess of the programmed level; (ii) in July-December 2009 upward by an amount equivalent to the lesser of total shortfalls of BPS (excluding that from the IMF) relative to programmed levels and CF 35,276 million; (iii) upward by an amount equivalent to any shortfall in debt relief under the MDRI from the IMF; and (iv) in January-December 2010 upward by an amount equivalent to the excess of external debt service payments relative to programmed amounts.

- Signing bonus from the SCCA: NDA will be adjusted (i) downward by an amount equivalent to total disbursement of signing bonus from SCCA in excess of the programmed levels; (ii) upward by an amount equivalent to total shortfalls in the disbursement of signing bonus from SCCA relative to programmed levels.

- Privatization proceeds: NDA will be adjusted (i) downward by the total amount of privatization proceeds (including PPCC) in excess of the programmed level; and (ii) in July-December 2009 upward by an amount equivalent to the lesser of total shortfalls in PPCC relative to programmed levels and CF 35,276 million.

\section{Ceiling on net banking system credit to the government (NCG)}

Definition: NCG is defined as the sum of net $\mathrm{BCC}$ and commercial bank claims on the central government, plus the BCC's net cash deficit. For purposes of program monitoring, government deposits related to externally financed projects are excluded from NCG. External 
budget support (BPS excluding balance of payment support from the IMF) will be converted to domestic currency by using the market exchange rate prevailing at the time of the disbursement.

10. The following adjustments will be made to the NCG ceiling.

- BPS: NCG will be adjusted (i) downward by an amount equivalent to total BPS (excluding that from the IMF) in excess of the programmed level, and (ii) upward by an amount equivalent to the lesser of total shortfalls in BPS (excluding that from IMF) relative to programmed levels and CF 35,276 million; and (iii) in JanuaryDecember 2010 upward by an amount equivalent to the excess of external debt service payments relative to programmed amounts.

- Signing bonus from the SCCA: NCG will be adjusted (i) downward by an amount equivalent to total disbursement of signing bonus from SCCA in excess of the programmed levels; (ii) upward by an amount equivalent to total shortfalls relative to programmed levels in the disbursement of signing bonus from SCCA.

- Privatization proceeds: the NCG ceiling will be adjusted (i) downward by an amount equivalent to total privatization proceeds in excess of the programmed levels, and (ii) in July-December 2009 upward by an amount equivalent to the lesser of total shortfalls in privatization proceeds relative to programmed levels and CF 35,276 million.

\section{Ceilings on new nonconcessional loans contracted or guaranteed by the central government, local governments, or the BCC}

11. Definition: With regard to contracted or guaranteed debt, the central government is defined as all units of government that exercise authority over the entire economic territory, including nonprofit organizations controlled and financed by the central government.

12. Definition: Debt is defined in point 9 of the Guidelines on Performance Criteria with Respect to Foreign Debt in IMF arrangements (Decision No. 12274-00/85 of August 24, 2000; see Annex).

\section{Ceilings on new medium- and long-term nonconcessional loans contracted or guaranteed by the central government, local governments, or the BCC}

13. Definition: nonconcessional debt is defined as all loans with a grant element of less than 35 percent, calculated as the difference between the present value (PV) of the debt and its nominal value, expressed as a percentage of the nominal value of the debt. The PV of the debt at the time it is contracted is calculated by discounting future flows of payable debt service. The discount rates used for that purpose are the commercial interest reference rates (CIRR) specific to each currency, published by the OECD. The average ten-year CIRRs will 
be used to calculate the PV of debt having a maturity of at least 15 years, and six-month average CIRRs for loans with shorter maturity. For the purposes of the program, the most recent CIRRs published by the OECD will be used to assess concessionality of loans.

14. Definition: the limit on nonconcessional medium- and long-term loans applies to contracted or guaranteed debt and liabilities for which the equivalent value has not been received. It excludes the use of IMF resources and debt contracted within the framework of a rescheduling or restructuring.

Ceilings on new nonconcessional short-term loans contracted or guaranteed by the central government, local governments, or the BCC

15. Definition: short-term debt is defined as debt having an initial maturity of one year or less, with the exception of regular import credits having a maturity of up to one year.

\section{E. Ceiling on the accumulation of external payment arrears}

16. Definition: external payment arrears include external debt service obligations (principal and interest) that were not paid on the contractual due date. The ceiling on new external payment arrears applies continuously throughout the period covered by the PRGF arrangement. It does not apply to external payment arrears in process of renegotiation or to cases in which the creditor has agreed to the suspension of payments pending the outcome of negotiations.

\section{QUANTITATIVE INDICATIVE TARGETS}

17. The indicative targets pertain to: (i) base money; (ii) the non-accumulation of wage arrears; and (iii) the domestic fiscal balance.

\section{A. Ceilings on base money}

18. Definition: base money is defined as the sum of (i) currency outside banks; (ii) cash holdings of commercial banks; (iii) deposits of commercial banks with the BCC; (iv) private sector deposits with the $\mathrm{BCC}$; (v) deposits of parastatal enterprises with the $\mathrm{BCC}$; and (vi) foreign exchange deposits and provisions for imports with the BCC.

\section{B. Ceilings on the accumulation of wage arrears}

19. Definitions: Wage arrears are defined as approved personnel expenses which have not been paid for 30 days. Wages include the total compensation paid employees (civil service; including permanent benefits). These arrears will be valued on a cumulative basis from January 1, 2009. 


\section{Ceiling on the domestic fiscal balance}

20. Definitions: the domestic fiscal balance is defined as (domestic revenue) minus (domestically financed expenditure). Domestic revenue is defined as (total revenue and grants) minus (grants) minus (signing bonus from the SCCA). Domestically financed expenditure is defined as (total expenditure and net loans) minus (externally financed investments) minus (foreign interest payments) plus (the BCC's operating deficit) plus (the net accumulation of domestic arrears).

21. The following adjustments will apply to the ceiling on the domestic fiscal balance:

- BPS: In July-December 2009, the floors on the domestic fiscal balance will be adjusted upward by an amount equivalent to the excess of total shortfall of BPS (excluding that from the IMF) relative to programmed levels over CF 35,276 million.

- Privatization proceeds: In July-December 2009, the floors on domestic fiscal balance will be adjusted upward by an amount equivalent to the excess of total shortfall of privatization proceeds relative to programmed levels over CF 35,276 million.

\section{Consultation Clause}

22. In the event that revenue exceeds the programmed amounts during the program period, the authorities will consult the IMF before allocating any surplus revenue to additional expenditure. Further, the authorities will consult with the IMF before implementing any revisions to the policies contained in the MEFP.

\section{Data to be Reported for Program Monitoring Purposes}

23. The authorities of the DRC will provide IMF staff with the data needed to monitor the program within the prescribed time limits, as indicated in the following table. 


\section{Summary of Data to be Reported}

\begin{tabular}{|c|c|c|c|}
\hline Information & Responsible entity & Frequency & $\begin{array}{l}\text { Reporting } \\
\text { deadline }\end{array}$ \\
\hline $\begin{array}{l}\text { Volume of foreign exchange purchases and } \\
\text { sales on the interbank market }\end{array}$ & $\mathrm{BCC}$ & Daily & One day \\
\hline $\begin{array}{l}\text { Volume of BCC purchases and sales on the } \\
\text { interbank market }\end{array}$ & $\mathrm{BCC}$ & Daily & One day \\
\hline $\begin{array}{l}\text { Average CGF/USD reference exchange } \\
\text { rate on the interbank market }\end{array}$ & $\mathrm{BCC}$ & Daily & One day \\
\hline $\begin{array}{l}\text { Average CGF/USD reference exchange } \\
\text { rate }\end{array}$ & $\mathrm{BCC}$ & Daily & One day \\
\hline $\begin{array}{l}\text { Average CGF/USD reference exchange } \\
\text { rate offered by commercial banks to their } \\
\text { customers }\end{array}$ & $\mathrm{BCC}$ & Daily & One day \\
\hline $\begin{array}{l}\text { Average CGF/USD reference exchange } \\
\text { rate used by exchange bureaus }\end{array}$ & $\mathrm{BCC}$ & Daily & One day \\
\hline Integrated monetary survey & $\mathrm{BCC}$ & Monthly & 2 weeks \\
\hline BCC balance sheet & $\mathrm{BCC}$ & Monthly & One week \\
\hline Monetary survey of retail banks & $\mathrm{BCC}$ & Monthly & 2 weeks \\
\hline Structure of retail banks' interest rates & $\mathrm{BCC}$ & Monthly & 2 weeks \\
\hline $\begin{array}{l}\text { Reserves (voluntary and required) of retail } \\
\text { banks }\end{array}$ & $\mathrm{BCC}$ & Daily & One day \\
\hline $\begin{array}{l}\text { Volume of CGF transactions on the } \\
\text { interbank market }\end{array}$ & $\mathrm{BCC}$ & Daily & One day \\
\hline $\begin{array}{l}\text { Outstanding central bank claims on retail } \\
\text { banks }\end{array}$ & $\mathrm{BCC}$ & Daily & One day \\
\hline $\begin{array}{l}\text { Foreign exchange sales (including through } \\
\text { auctions) by the BCC }\end{array}$ & $\mathrm{BCC}$ & Weekly & One week \\
\hline $\begin{array}{l}\text { Outstanding and new issues of central bank } \\
\text { bills (BTR) }\end{array}$ & $\mathrm{BCC}$ & Weekly & One day \\
\hline Change in the free reserves of banks & $\mathrm{BCC}$ & Weekly & One day \\
\hline Structure of BCC interest rates & $\mathrm{BCC}$ & Monthly & One week \\
\hline Consumer price index & $\mathrm{BCC}$ & Weekly & One week \\
\hline $\begin{array}{l}\text { Retail banks' financial soundness } \\
\text { indicators }\end{array}$ & $\mathrm{BCC}$ & Monthly & 2 weeks \\
\hline $\begin{array}{l}\text { Commodity exports (value and volume), } \\
\text { imports (value and volume) and domestic } \\
\text { production indicators }\end{array}$ & $\mathrm{BCC}$ & Monthly & 3 weeks \\
\hline $\begin{array}{l}\text { Implementation of the } \mathrm{BCC} \text { foreign } \\
\text { exchange cash flow plan }\end{array}$ & $\mathrm{BCC}$ & Weekly & 1 week \\
\hline
\end{tabular}




\section{Summary of Data to be Reported (Concluded)}

\begin{tabular}{|c|c|c|c|}
\hline Information & Responsible entity & Frequency & $\begin{array}{l}\text { Reporting } \\
\text { deadline }\end{array}$ \\
\hline $\begin{array}{l}\text { Implementation of the BCC domestic } \\
\text { currency cash flow plan }\end{array}$ & $\mathrm{BCC}$ & Monthly & 1 week \\
\hline $\begin{array}{l}\text { Amounts and holders of promissory notes } \\
\text { (bills) guaranteed by the BCC }\end{array}$ & $\mathrm{BCC}$ & Monthly & 3 weeks \\
\hline $\begin{array}{l}\text { Evolution of Commitment Plan and } \\
\text { Treasury Plan Implementation }\end{array}$ & $\mathrm{MF} / \mathrm{MB}$ & Weekly & One day \\
\hline $\begin{array}{l}\text { Implementation of the government cash } \\
\text { flow plan }\end{array}$ & MF & Monthly & 2 weeks \\
\hline $\begin{array}{l}\text { Amount, terms, holders, and stock of } \\
\text { promissory notes (bills) }\end{array}$ & $\mathrm{MF} / \mathrm{BCC}$ & Monthly & 3 weeks \\
\hline $\begin{array}{l}\text { Breakdown of customs and excise } \\
\text { revenues }\end{array}$ & MF & Monthly & 4 weeks \\
\hline Breakdown of direct and indirect taxes & MF & Monthly & 4 weeks \\
\hline Breakdown of nontax revenues & MF & Monthly & 4 weeks \\
\hline $\begin{array}{l}\text { Projected expenditure commitment } \\
\text { schedule }\end{array}$ & MB & Quarterly & 2 weeks \\
\hline Budgetary monitoring statement (ESB) & MB & Monthly & 2 weeks \\
\hline Approved wage bill by type of beneficiary & MF & Monthly & 3 weeks \\
\hline Wage bill paid by type of beneficiary & MF & Monthly & 3 weeks \\
\hline Compensated employees by category & MF & Monthly & 3 weeks \\
\hline Civil service wage scale & MF & $\begin{array}{l}\text { In the event of } \\
\text { change }\end{array}$ & 3 weeks \\
\hline $\begin{array}{l}\text { Amounts of emergency spending, amounts } \\
\text { approved by the emergency spending } \\
\text { committee, amounts adjusted and paid by } \\
\text { the BCC }\end{array}$ & $\mathrm{MF} / \mathrm{BCC}$ & Monthly & 3 weeks \\
\hline Privatization receipts & $\mathrm{MF} / \mathrm{BCC}$ & Monthly & 3 weeks \\
\hline $\begin{array}{l}\text { Public sector domestic debt by category } \\
\text { and by creditor }\end{array}$ & MF & Monthly & 3 weeks \\
\hline $\begin{array}{l}\text { Loan contracts for any new external debt } \\
\text { contracted or guaranteed by the central } \\
\text { government, the BCC and local } \\
\text { governments }\end{array}$ & $\mathrm{MF} / \mathrm{BCC}$ & Monthly & 3 weeks \\
\hline $\begin{array}{l}\text { Budget execution monitoring table } \\
\text { showing Annual Treasury and } \\
\text { Commitment Plans and all stages of } \\
\text { expenditure execution through payments. }\end{array}$ & $\mathrm{MF} / \mathrm{MB}$ & Weekly & 3 days \\
\hline $\begin{array}{l}\text { Price Waterhouse Coopers audit reports, } \\
\text { indicating any adjustments made to data } \\
\text { reported at test dates }\end{array}$ & $\mathrm{BCC}$ & & 1 week \\
\hline
\end{tabular}




\section{Annex \\ Definition of debt}

Debt is defined as direct, and therefore unconditional obligation created under a contractual agreement providing for the provision of value in the form of assets (including monetary) or services, which requires the debtor to make one or more payments in the form of assets (including monetary) or to provide services on one or more future dates; these payments discharge the principal and interest liabilities incurred by the debtor under the contract. Debt can take a number of forms, including: (i) loans, that is, advances of funds to the debtor by the creditor on the basis of an undertaking that the debtor will repay the funds in the future (including deposits, bonds, debentures, commercial loans, and buyers' credits) and temporary exchanges of assets that are equivalent to fully collateralized loans, under which the debtor is required to repay the loaned funds and usually pay interest, by repurchasing in the future the assets given as collateral to the seller (for example, repurchase agreements or official swap arrangements); (ii) suppliers' credits, that is, contracts whereby the supplier permits the customer to defer payment until sometime after the date on which the goods are delivered or the services are provided; and (iii) leases, that is, arrangements giving the lessee the right to use a property for periods of time that are usually shorter than the useful life of the property in question, but without transferring ownership, title to which is retained by the lessor. For the purposes of this directive, the debt is the present value (at the inception of the lease) of all lease payments expected to be made during the term of the arrangement, excluding payments covering the operation, repair, and maintenance of the properties in question.

Under the definition of debt set out above, arrears, penalties, and court-awarded damages arising from a failure to make payment under a contractual obligation having the character of debt also constitute debt. Failure to make payment on an obligation that is not considered debt under this definition (e.g., payment on delivery) will not give rise to debt. 


\title{
INTERNATIONAL MONETARY FUND AND \\ INTERNATIONAL DEVELOPMENT ASSOCIATION
}

\author{
DEMOCRATIC REPUBLIC OF CONGO
}

\author{
Joint IMF/World Bank Debt Sustainability Analysis 2009 \\ Prepared by the Staffs of the International Monetary Fund and \\ the International Development Association \\ Approved by Mark Plant and Dominique Desruelle (IMF) and \\ Carlos Alberto Braga and Sudhir Shetty (IDA)
}

November 30, 2009

This note provides an updated debt sustainability analysis (DSA) for the Democratic Republic of the Congo (DRC) using the joint Bank-Fund Debt Sustainability Framework (DSF) for Low Income Countries. The results indicate that the DRC remains in debt distress, as was the case in the most recent DSA conducted in August 2007. In the absence of debt relief provided under the enhanced Heavily Indebted Poor Country (HIPC) Initiative and Multilateral Debt Relief Initiative (MDRI), external debt burden indicators are projected to remain above policy-dependent thresholds over prolonged periods. Even after HIPC/MDRI debt relief, the external debt outlook would be vulnerable to adverse shocks. External borrowing to finance public infrastructure projects under the Sino-Congolese Cooperation Agreement (SCCA) are projected to increase significantly the country's external debt burden indicators over the medium term, but related risks to debt sustainability appear manageable.

24. The Democratic Republic of the Congo (DRC) remains in debt distress. At end 2008 the present value of public and publicly guaranteed (PPG) external debt is estimated at 93 percent of GDP, 150 percent of exports and 501 percent of government revenue (excluding grants), well above policy-dependent threshold levels. ${ }^{10}$ Moreover, all debt burden indicators are projected to worsen significantly in 2009 due to marked declines in GDP, exports and government revenue in the wake of the global economic slowdown and accompanying sharp decline in world commodity prices.

\section{BACKGROUND}

25. The DRC continues to accumulate external arrears mainly on debt owed to bilateral creditors. The DRC reached the decision point of the enhanced HIPC Initiative in

\footnotetext{
${ }^{10}$ With an average CPIA rating of 2.80 in $2006-08$, the DRC is classified having a weak policy framework.
} 
July 2003, qualifying for interim debt relief. ${ }^{11}$ The IMF discontinued interim assistance in June 2006 when the PRGF program went off track. The Paris Club agreed to reschedule DRC's debt under Cologne flow terms in November 2003, which was also suspended in June 2006 when the PRGF program went off track. The International Development Agency's (IDA) interim HIPC assistance expired in October 2009. The African Development Bank/Fund will deliver interim assistance in 2010. The nominal value of PPG external debt (including arrears) is estimated at US\$13.1 billion at end-2008, US\$7.1 billion of which is owed to bilateral creditors, US\$4.4 billion to multilateral institutions, and US $\$ 1.5$ billion to commercial creditors. Arrears are estimated at US\$3.3 billion at end-2008, of which US\$1.5 billion is owed to Paris Club creditors.

\section{External Debt SustainabiLity Analysis}

26. The baseline scenario in this DSA assumes a strong recovery in economic activity over the medium term, supported by large investments in mining and public infrastructure projects. In particular, it assumes that the security situation in the eastern provinces stabilizes and that the government adopts prudent macroeconomic policies and makes significant progress on key structural reforms, including strengthening tax collection, improving budget preparation and execution, and reforming the civil service. The near-term outlook is dominated by the impact of the global economic slowdown (Box 1), followed by a strong recovery over the medium term fueled by a US\$3.2 billion investment in a mining project along with US\$3 billion in investments in public infrastructure projects envisaged under the SCCA (Box 2).

27. The baseline scenario assumes that a new three-year PRGF arrangement will be in place by the end of 2009, which will allow the IMF and the DRC's Paris Club creditors to provide additional interim assistance under the enhanced HIPC Initiative. ${ }^{12}$ It also assumes that arrears to bilateral creditors are rescheduled or deferred, the African Development Bank/Fund delivers interim HIPC assistance in 2010, and that IDA provided interim HIPC assistance up to October 2009.

\footnotetext{
11 "Democratic Republic of the Congo-Decision Point Document for the Enhanced Heavily Indebted Poor Countries (HIPC) Initiative”, and IDA/R2003-0059.

12 The baseline scenario does not assume that the DRC reaches the HIPC completion point in accordance with current LIC DSA guidelines outlined in: "Staff Guidance Note on the Application of the Joint Fund-Bank Debt Sustainability Framework for Low-Income Countries", April 2007and IDA/No. 39748. An alternative scenario assumes that the DRC reaches the HIPC completion point in mid-2010.
} 


\section{Box 1. Democratic Republic of the Congo: Macroeconomic Assumptions for the DSA}

Although short-term economic conditions remain weak, the medium-term outlook is positive. The baseline projection scenario has the following key elements.

- GDP growth declines from 6.2 percent in 2008 to 2.7 percent in 2009 due to the slowdown in global growth and the sharp decline in world commodity prices from record levels reached in mid-2008. Growth rebounds strongly over the medium term, averaging 7.5 percent over the period 2010-16, spurred by large investment expenditures under the SCCA (Box 2), and a recovery in mining activity. The mining project financed by the SCCA is projected to increase real GDP growth by 0.3 percentage points on average during the construction phase 2009-12 and by 0.6 percentage points on average during the production phase in 2013-16. The public infrastructure projects financed by SCCA are projected to increase real GDP growth by 0.7 percentage points on average during the construction phase 2009-12, declining to 0.2 percentage points in the long run (Box 3).

- Inflation declines to 48.7 percent by end-2009 and to single digits over the medium term, supported by prudent fiscal and monetary policies.

- The overall fiscal deficit (including grants) widens substantially in the short run, reaching 12 percent in 2010, before narrowing gradually to around 2 percent of GDP in the long run as ongoing reforms expand the tax base and noninterest expenditures decline slowly as a percent of GDP. Grants decline from 8.7 percent of GDP in 2009 to under 4 percent in 2018.

- The current account deficit widens from 15 percent of GDP in 2008 to 32 percent in 2011 mainly due to a large increase in imported factors used in the mining and public infrastructure projects. The deficit narrows thereafter as mining exports expand and investment-related imports diminish, stabilizing at around 6 percent of GDP over the long run.

- $\quad$ External financing over the medium term is dominated by loans disbursed to fund public infrastructure projects under the SCCA, accounting for 22 percent of gross borrowing needs over the period 2009-14. The average grant element of new borrowing rises steadily from 20 percent in 2009 to 43 percent in 2015 and beyond.

- $\quad$ Net foreign direct investment declines slightly from an average level of 6.3 percent of GDP in 2004-08 to 5.8 percent in 2009 in the wake of the global economic downturn, recovering to an average level of 7.2 percent of GDP by 2010-12 led by foreign investment in mining projects. 


\section{Box 2. Democratic Republic of the Congo: The Sino-Congolese Cooperation Agreement (SCCA)}

In April 2008, the DRC signed a cooperation agreement between a Congolese parastatal mining enterprise (GECAMINES) and a consortium of Chinese enterprises, forming a joint venture company (SICOMINES). The net operating income from the joint venture is to be distributed by equity shares with 32 percent going to GECAMINES and 68 percent to the consortium of Chinese enterprises.

The original agreement (signed in April 2008) included a US\$3.2 billion mining project, along with US\$6 billion in public infrastructure projects to be implemented in two phases over the period 2009-17. The agreement was amended in October 2009 to exclude the second phase of public infrastructure projects totaling US\$3 billion that was to be implemented over the period 2014-17, leaving just the first phase of public infrastructure projects totaling US\$3 billion to be implemented over the period 2009-13.

Under the original agreement, the Congolese government provided a public guarantee on the loans for the mining and public infrastructure projects. However, the public guarantee on the mining loan was removed in the amended agreement. The public guarantee requires that the Congolese government repay any principal outstanding on the public infrastructure loans at end 2034 (25 years after the formation of the joint venture SICOMINES) with capitalized interest.

The US\$3.2 billion mining investment is to be financed by a US\$1.1 billion interest-free loan along with a US\$2.1 billion loan with an interest rate of 6.1 percent. The US\$3 billion investment in public infrastructure is to be financed by a series of loans to be disbursed for the individual projects, each with a fixed interest rate of 4.4 percent (LIBOR plus 100 basis points as of April 22, 2008). All loans are denominated in US dollars.

The consortium of Chinese enterprises provides the DRC government with a US\$250 million signing bonus ("pas de porte"), half of which was disbursed in March 2009. The remaining balance will be disbursed upon the transfer of the mining concession title to SICOMINES. GECAMINES receives a US\$100 million signing bonus, also disbursed in two tranches.

The mining project is expected to generate operating profits beginning in 2013, which will be used to repay the mining and public infrastructure loans in three stages. In the first stage, all profits from the mine will go to repay principal and capitalized interest on US\$375 million in "urgent" public infrastructure loans. In the second stage, 85 percent of operating profits will be used to repay the mining loans. In the third stage, 85 percent of operating profits will be used to repay the remaining public infrastructure loans. In the second and third stages, 15 percent of operating profits will be paid to the shareholders of the joint venture SICOMINES. 


\section{Box 3. The Democratic Republic of the Congo: The Economic Impact of Public Infrastructure Projects}

A two-stage approach was used to analyze the long-run growth impact of the US\$3 billion in public infrastructure projects to be undertaken under the SCCA. ${ }^{1}$ In the first stage, the analysis identified the location and size of current economic activities (e.g., agriculture, forestry, and mining) and the locationspecific economic potential. Location-specific transport costs take the distance to relevant markets into account using a "cost-distance" algorithm. Sector-specific responses to changes in transport costs were estimated and used to simulate the long-term impact of transport investments on production location levels. Long-term elasticities based on cross-sectional reduced-form regressions were used to estimate the response of local production to a change in transportation costs. In a second stage, a multiplier approach was used to gauge the demand side effects of public infrastructure projects during the implementation phase. The analysis indicates that the public infrastructure projects would raise real GDP growth by 0.7 percentage points on average during the construction phase 2009-13 mainly through the impact of the higher investment on domestic demand. The growth impact declines to 0.2 percentage points upon completion of the projects reflecting gains in total factor productivity associated with the improved public infrastructure.

See World Bank (2009, forthcoming), "Prioritizing Infrastructure Investments: A Spatial Approach”.

\section{Under the baseline scenario, external debt burden indicators breach} policy-dependent thresholds over prolonged periods (Figure 1). The present value of PPG external debt exceeds the 30 percent of GDP threshold over most of the 20 -year projection period (Figure 1b), notwithstanding the strong recovery projected over the medium term. The debt-to-export and debt-to-revenue ratio indicators decline somewhat faster over the projection period, falling below their thresholds in 2018 and 2019, respectively (Figures 1c and 1d). This reflects higher export revenues resulting from the large mining projects and higher tax revenue ensuing from reforms undertaken to broaden the tax base.

29. The baseline projections are subject to significant downside risks. Given the DRC's high dependence on mining exports, the external account and overall economic outlook remains highly vulnerable to adverse terms of trade shocks. Moreover, poor governance, unresolved security issues, weak implementation capacity and lower than envisaged donor support could also worsen the external debt outlook significantly.

30. All debt burden indicators worsen substantially under standard stress tests. The debt profile is most vulnerable to a shock that entails public sector debt financed under less favorable terms than assumed in the baseline (Figure 1). ${ }^{13}$ The external debt burden indicators decline somewhat more rapidly under the historical scenario. This reflects the fact

\footnotetext{
${ }^{13}$ The average grant element on net borrowing is only 30 percent in the period 2009-13, well below the 35 percent grant element required to qualify as concessional. The large difference reflects two main factors. First, the concessionality calculation is based on a much higher discount rate than that used in the DSA. Second, the DSA includes refinancing of arrears to bilateral creditors on non-concessional terms.
} 
that the country had no access to external financing during the conflict period which restricted the noninterest current account deficit.

\section{The external debt burden indicators are projected to breach their thresholds} even if the country were to receive debt relief under the enhanced HIPC Initiative and the MDRI. If the DRC were to reach the completion point in mid-2010, all three external debt burden indicators breach their thresholds (Figure 2). The external debt-to-GDP ratio breaches the 30 percent threshold over the period 2010-18 reaching a high of 45 percent in 2012 (Figure 2b). The external debt-to-revenue ratio breaches the 200 percent threshold slightly in just two years (2012 and 2014). New purchases under the proposed PRGF arrangement would account for 6.5 percent of the PV of external PPG debt in 2012 (2.9 percent of GDP), declining to 2.7 percent in 2019 ( 0.7 percent of GDP). The external debt profiles are robust to lower long-run growth projections. Reducing real GDP growth by half of a percentage point after 2017 (which roughly offsets the assumed benefit from the public infrastructure projects) raises the PV of the PPG external debt-to-GDP ratio by only 1.3 percentage points in 2029. The debt profiles are vulnerable to adverse shocks, however. In particular, two of the three external debt burden indicators breach their respective thresholds over the entire projection period under the most extreme stress test (Figures $2 \mathrm{~b}$ and $2 \mathrm{~d}$ ).

\section{The public guarantee on the infrastructure financing under the SCCA raises the} external debt burden indicators over the medium term but does not have a major impact on debt sustainability over the long term. Removing the public guarantee on the loans for the public infrastructure projects would reduce the external debt-to-GDP ratio by 8.6 percentage points on average over the period 2011-16, but would have no impact after 2019 when the loans are projected to be fully repaid (Figure 3). ${ }^{14}$ The present-value calculations underlying the DSA take into account that the public guarantee can only be invoked after 25 years. ${ }^{15}$ If the public guarantee were based on a conventional loan agreement with a fixed repayment schedule equal to the projected payments from the net operating income of SICOMINES, ${ }^{16}$ the external debt burden indicators would be just slightly higher over medium term, but with no impact beyond 2019 (Figure 3).

\footnotetext{
${ }^{14}$ The DSA includes the public guarantee on the infrastructure financing made under the SCCA as external PPG debt, but does not include the debt service payments made by the net operating profit from the joint venture SICOMINES.

${ }^{15}$ To illustrate, consider the following example. In 2009, the Chinese consortium is projected to disburse a total of $\$ 375$ million in loans for public infrastructure projects. The legal obligation of the government under the terms of the agreement is to repay any outstanding balance (principal and compounded interest) in 2034. In keeping with the conventional for valuing public guarantees, we assume the worst outcome-zero operating income over the entire 25-year period. This implies a legal obligation of $\$ 375$ million in principal and $\$ 725$ million in compounded interest in 2034, which has a present value of \$397 million in 2009 (based on a discount rate of 4 percent used in DSAs for low-income countries).

${ }^{16}$ For example, the $\$ 375$ million in loans to be disbursed in 2009 is projected to be fully repaid (principal and capitalized interest) in 2013. This would raise the present value of external debt by $\$ 426$ million in the case of a
} 


\section{Public Debt Sustainability Analysis}

\section{The DRC's domestic debt is relatively low and as a result, the above external} debt sustainability analysis broadly applies to total public debt, albeit with slightly higher debt burden indicators (Figure 4). Although information on domestic debt is poor, the authorities recently provided updated estimates on claims of about US\$1.3 billion at end-2008 (11 percent of GDP). These claims are mainly from suppliers, public enterprises and public sector employees dating back to the period of conflict in the 1990s. The authorities are in the process of developing a strategy — to be finalized in mid 2010 - to address with these claims.

\section{CONCLUSION}

\section{The DRC would remain in debt distress in the absence of substantial debt relief.}

The external debt indicators would breach policy-dependent thresholds over prolonged periods even with a strong economic recovery over the medium term (assumed under the baseline scenario). Even if the DRC were to reach the HIPC completion point in mid-2010 and benefit from HIPC/MDRI debt relief, the external debt outlook remain vulnerable to adverse shocks. The public guarantee on the SCCA financing increases the external debt-toGDP ratio significantly above the policy-dependent threshold level for a prolonged period. However, these calculations substantially overstate the risk of debt distress because they assume that the mining project will not generate any net operating profits over the entire 25-year projection period. Calculations based on a model of income generated by the mining project developed by the DRC authorities predict that the net operating profits from the mining project would fully repay the public infrastructure loans by 2018, 16 years before the public guarantee would be invoked. Net operating income would have to decline by 65 percent of the amount projected in the baseline scenario in order for the public guarantee to be invoked. These calculations indicate that the risk for debt sustainability is manageable.

conventional loan with a fixed repayment schedule, compared to only $\$ 267$ million in the case where the public guarantee can only be invoked after 25 years. 
Figure 1. Democratic Republic of the Congo: Indicators of Public and Publicly Guaranteed External Debt without Debt Relief, 2009-29

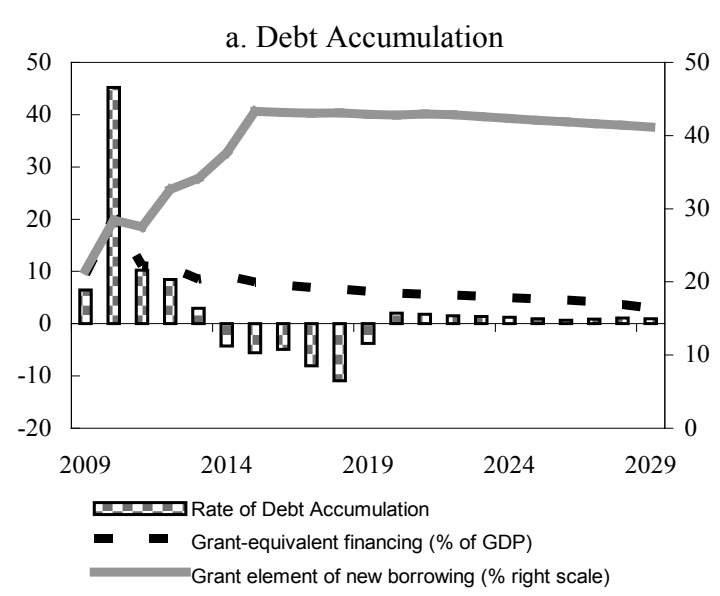

c. PV of debt-to-exports ratio
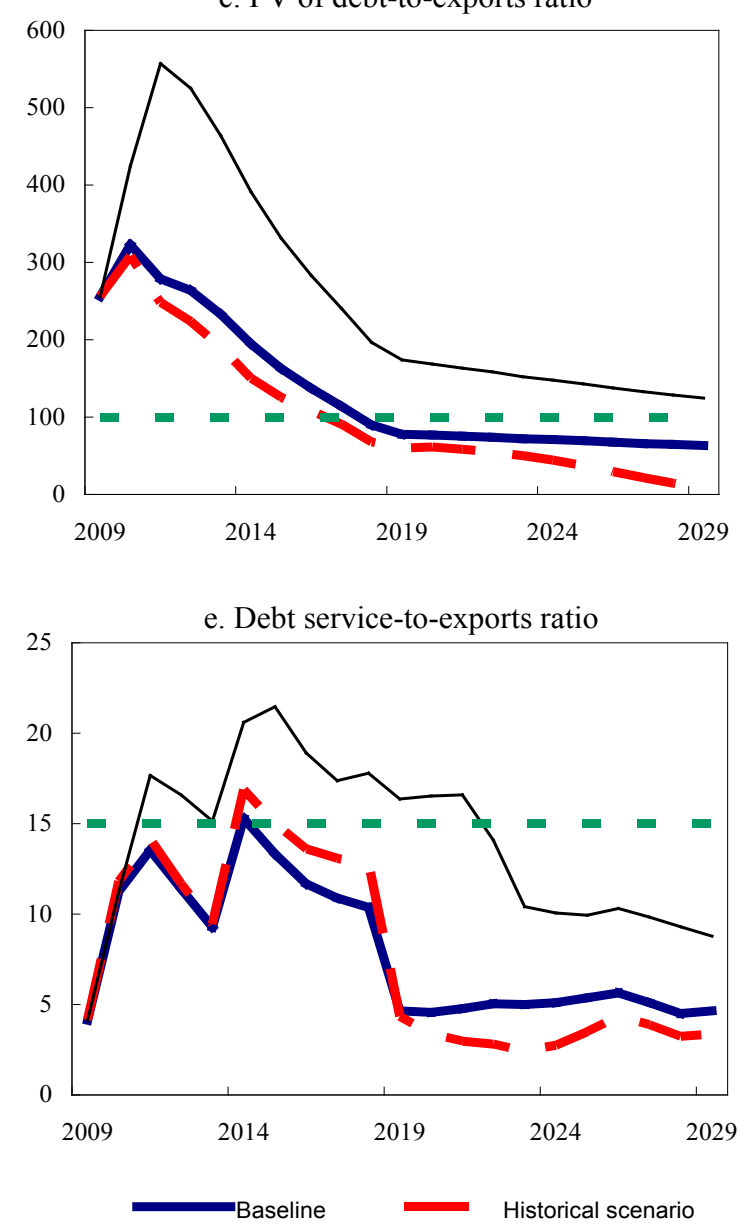

Source: IMF staff projections and simulations. b. PV of debt-to-GDP ratio

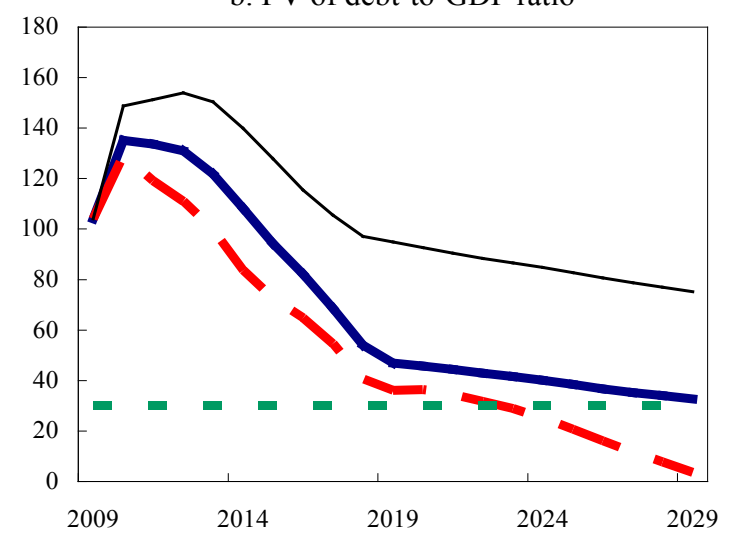

d. PV of debt-to-revenue ratio
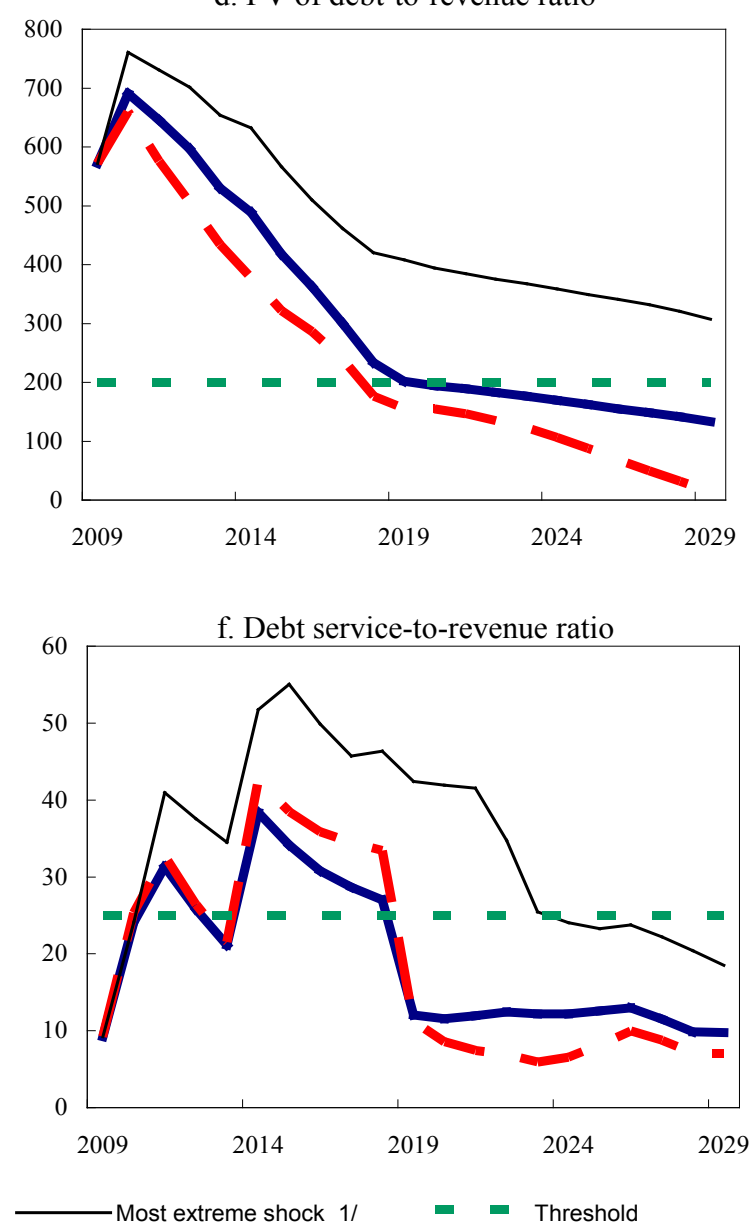

$1 /$ The most extreme stress test is the test that yields the highest ratio in 2019, which is new public sector loans on less favorable terms in figures $b, d$ and $f$ and lower exports in figures $c$ and $e$. 
Figure 2. Democratic Republic of the Congo: Indicators of Public and Publicly Guaranteed External Debt - HIPC Completion Point Scenario, 2009-29
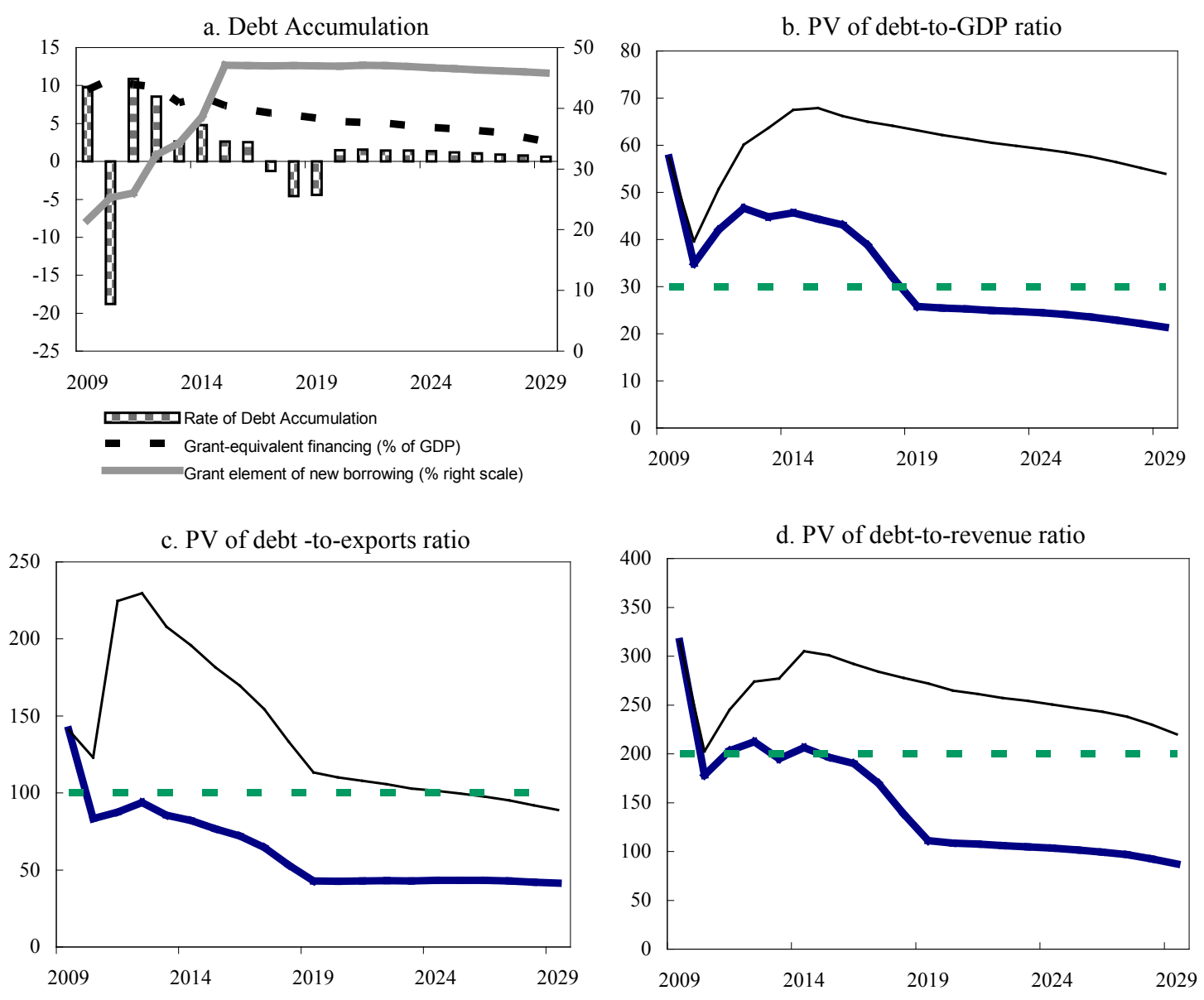

e. Debt service-to-exports ratio
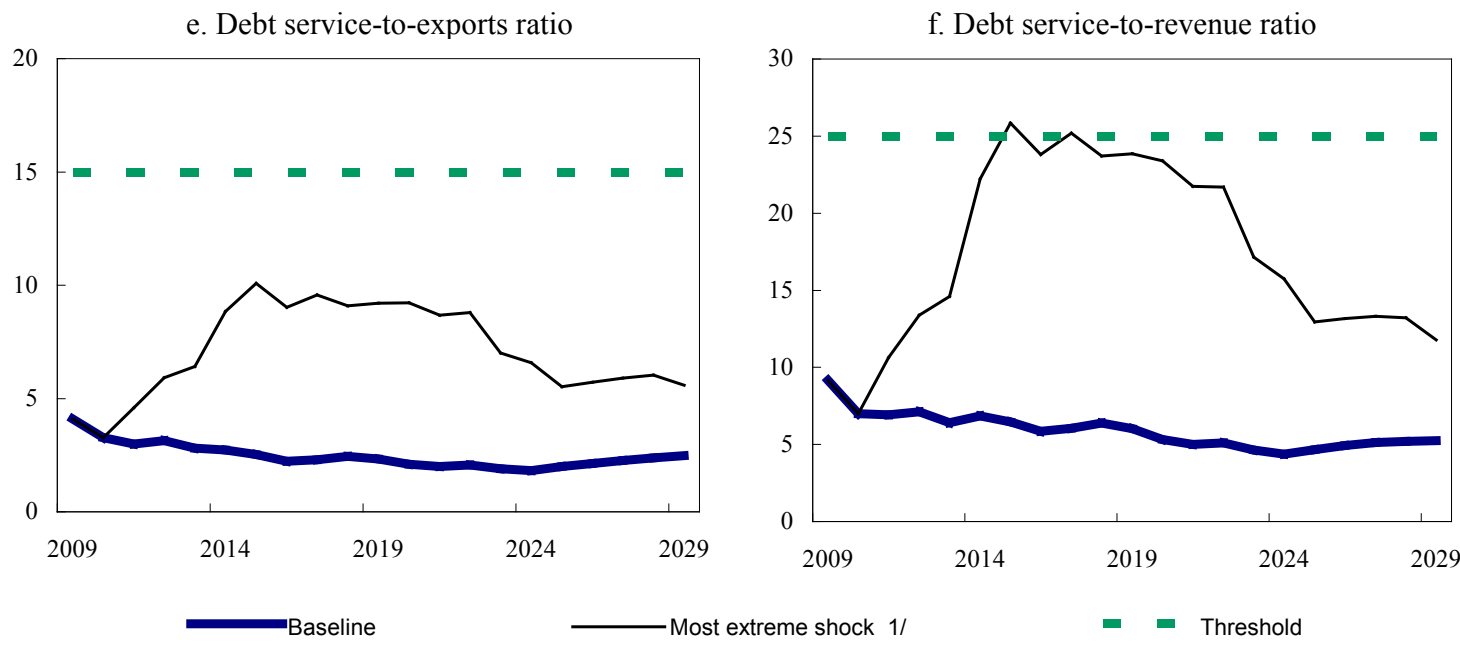

Source: IMF staff projections and simulations.

$1 /$ The most extreme stress test is the test that yields the highest ratio in 2019 , which is new public sector loans on less favorable terms in figures $b$ to $f$. 
Figure 3. Democratic Republic of the Congo: Indicators of Public and Publicly Guaranteed External Debt HIPC Completion Point Scenario with Alternative Valuations of Public Guarantee, 2009-29

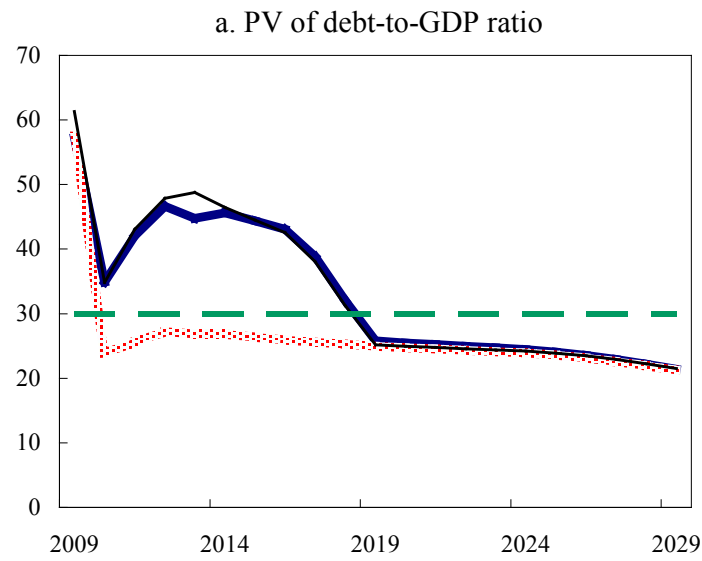

b. PV of debt-to-exports ratio

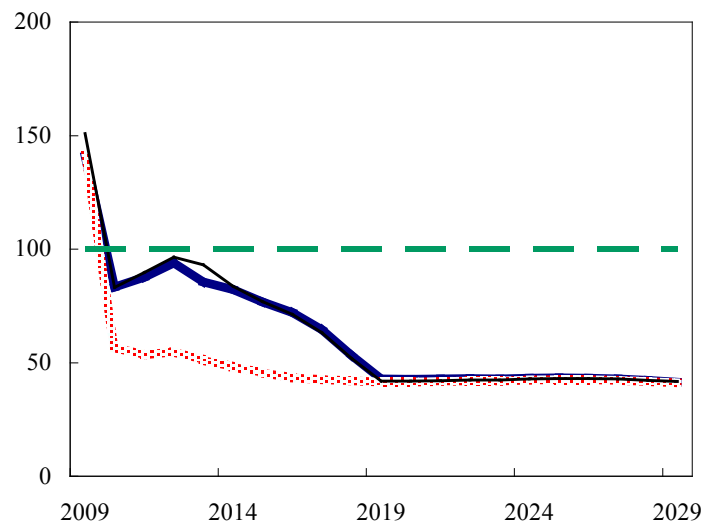

d. Debt service-to-exports ratio

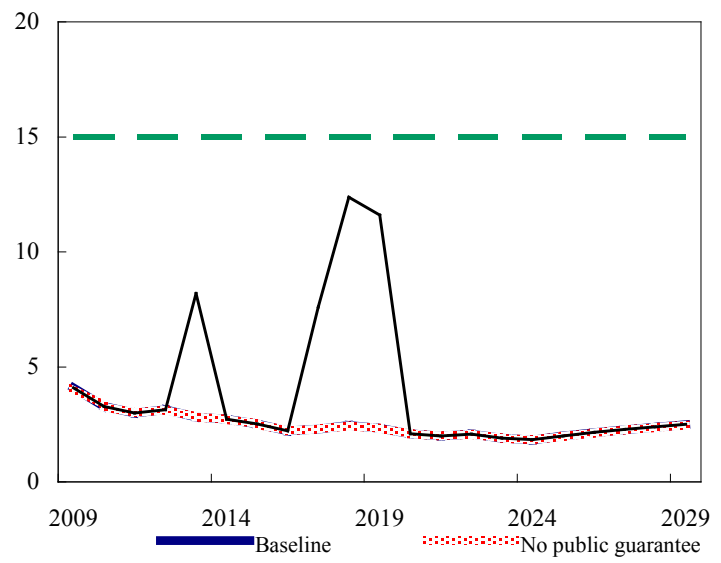

c. PV of debt-to-revenue ratio
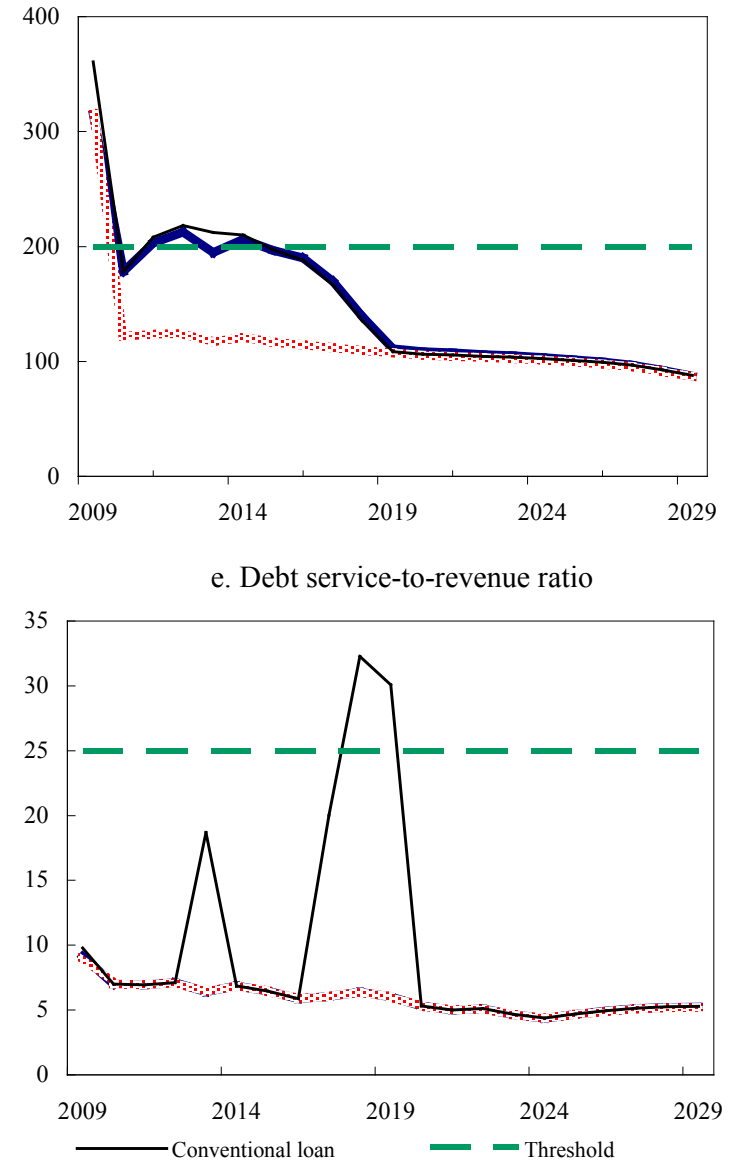

Sources: Congolese authorities; and IMF staff estimates and projections. 
Figure 4. Democratic Republic of the Congo: Indicators of Public Debt without Debt Relief, 2009-29

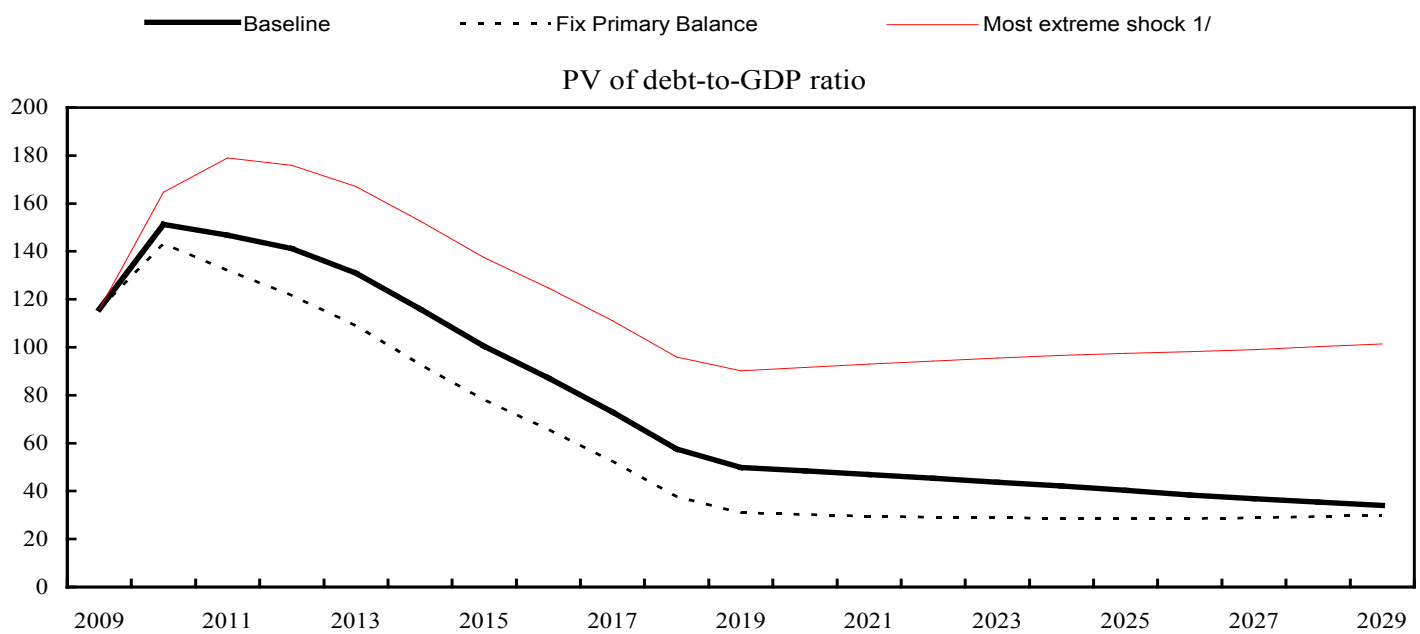

PV of debt-to-revenue ratio

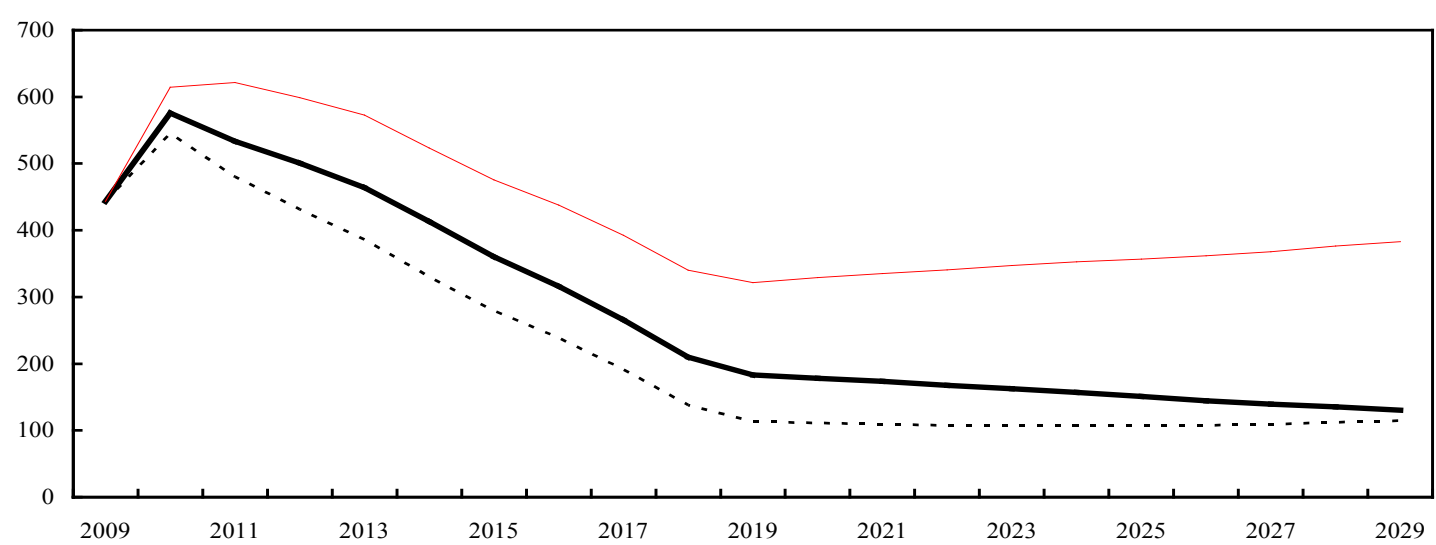

Debt service-to-revenue ratio $2 /$

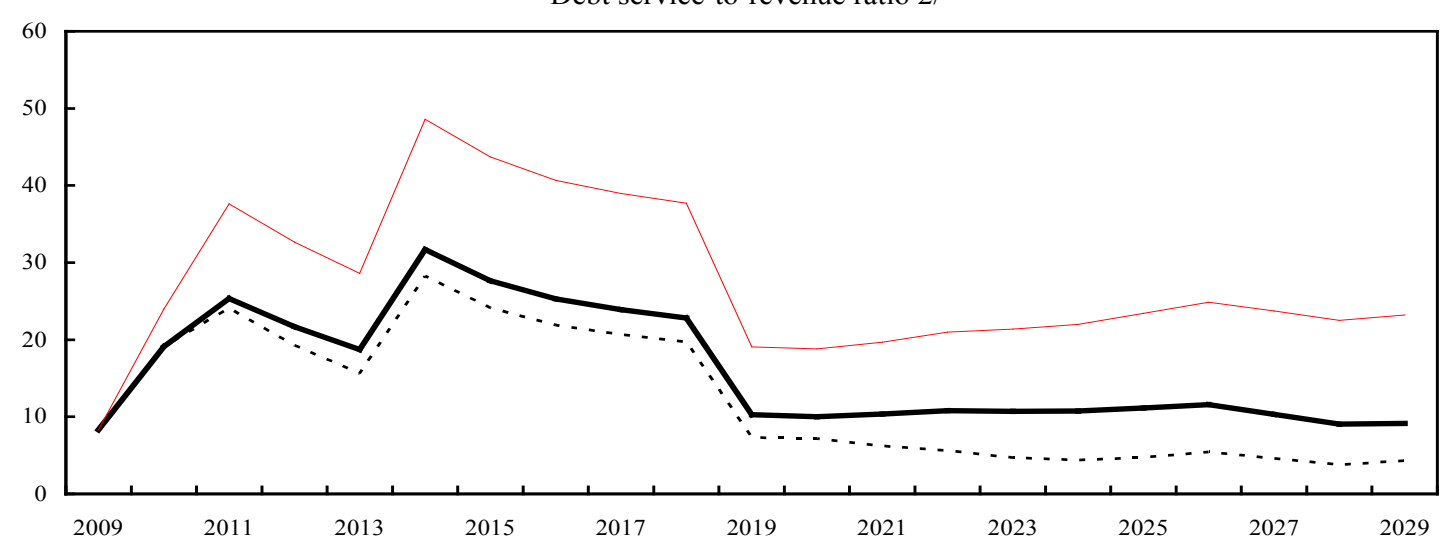

Sources: Congolese authorities; and IMF staff estimates and projections.

1/ The most extreme stress test is the test that yields the highest ratio in 2019.

2/ Revenues are defined inclusive of grants. 
Table 1. Democratic Republic of the Congo: External Debt Sustainability Framework, Baseline Scenario, 2008-29 1/ (In percent of GDP, unless otherwise indicated)

\begin{tabular}{|c|c|c|c|c|c|c|c|c|c|c|c|c|c|}
\hline & \multirow{2}{*}{$\begin{array}{r}\text { Actual } \\
2008 \\
\end{array}$} & \multirow{2}{*}{$\begin{array}{l}\text { Historical } \\
\text { Average }\end{array}$} & \multirow{2}{*}{$\begin{array}{l}\text { Standard } \\
\text { Deviation }\end{array}$} & \multicolumn{6}{|c|}{ Projections } & \multirow[b]{2}{*}{$\begin{array}{l}2009-14 \\
\text { Average }\end{array}$} & \multirow[b]{2}{*}{2019} & \multirow[b]{2}{*}{2029} & \multirow[b]{2}{*}{$\begin{array}{l}2015-29 \\
\text { Average }\end{array}$} \\
\hline & & & & 2009 & 2010 & 2011 & 2012 & 2013 & 2014 & & & & \\
\hline External debt (nominal) 1/ & 128.4 & & & 138.7 & 154.2 & 165.8 & 170.2 & 164.7 & 157.5 & & 77.7 & 54.0 & \\
\hline $\mathrm{o} / \mathrm{w}$ public and publicly guaranteed (PPG) & 128.4 & & & 132.4 & 138.8 & 139.1 & 138.1 & 131.0 & 123.0 & & 74.9 & 54.0 & \\
\hline Change in external debt & 3.8 & & & 10.3 & 15.4 & 11.6 & 4.5 & -5.6 & -7.2 & & -12.8 & -2.1 & \\
\hline Identified net debt-creating flows & -19.1 & & & 4.8 & 9.7 & 7.8 & 2.8 & -2.6 & -3.7 & & -6.0 & 2.3 & \\
\hline Non-interest current account deficit & 12.7 & 2.6 & 4.9 & 13.7 & 21.2 & 23.2 & 17.6 & 13.7 & 10.4 & & 2.7 & 6.9 & 5.6 \\
\hline Deficit in balance of goods and services & 14.9 & & & 23.8 & 27.9 & 31.5 & 24.6 & 18.6 & 14.3 & & 2.6 & 6.1 & \\
\hline Exports & 61.6 & & & 40.6 & 41.8 & 48.0 & 49.7 & 52.4 & 55.6 & & 60.2 & 51.7 & \\
\hline Imports & 76.4 & & & 64.5 & 69.8 & 79.4 & 74.2 & 70.9 & 69.8 & & 62.7 & 57.8 & \\
\hline Net current transfers (negative $=$ inflow) & -10.6 & -6.7 & 2.6 & -13.9 & -10.0 & -9.5 & -8.7 & -7.1 & -6.5 & & -5.1 & -3.7 & -4.7 \\
\hline o/w official & -8.8 & & & -12.8 & -9.4 & -8.8 & -8.0 & -6.4 & -5.7 & & -3.9 & -2.2 & \\
\hline Other current account flows $($ negative $=$ net inflow) & 8.5 & & & 3.8 & 3.3 & 1.2 & 1.7 & 2.2 & 2.7 & & 5.2 & 4.4 & \\
\hline Net FDI (negative = inflow) & -14.8 & -4.8 & 4.3 & -5.7 & -6.5 & -8.4 & -7.1 & -6.1 & -6.1 & & -5.7 & -2.7 & -4.6 \\
\hline Endogenous debt dynamics 21 & -17.0 & -4.0 & 4.0 & $\begin{array}{l}-3.1 \\
-3.3\end{array}$ & -5.0 & -7.0 & -7.6 & -10.2 & -8.0 & & $\begin{array}{l}-3.1 \\
-3.0\end{array}$ & -1.8 & -4.0 \\
\hline Contribution from nominal interest rate & 0.3 & & & 0.3 & 1.8 & 3.3 & 2.9 & 2.6 & 2.4 & & 1.1 & 0.5 & \\
\hline Contribution from real GDP growth & -6.6 & & & -3.6 & -6.7 & -10.2 & -10.5 & -12.8 & -10.4 & & -4.1 & -2.3 & \\
\hline Contribution from price and exchange rate changes & -10.7 & & & & & & & & & & & & \\
\hline $\begin{array}{l}\text { Residual (3-4) } 3 / \\
\text { o/w excoptional financing }\end{array}$ & $\begin{array}{l}22.9 \\
-60\end{array}$ & & & $\begin{array}{r}5.6 \\
-7.3\end{array}$ & $\begin{array}{r}5.7 \\
-356\end{array}$ & $\begin{array}{r}3.8 \\
-4.3\end{array}$ & $\begin{array}{r}1.6 \\
-3.4\end{array}$ & $\begin{array}{l}-2.9 \\
-2.7\end{array}$ & $\begin{array}{r}-3.4 \\
2.0\end{array}$ & & $\begin{array}{l}-6.8 \\
-12\end{array}$ & $\begin{array}{l}-4.4 \\
-0.4\end{array}$ & \\
\hline $\begin{array}{l}\text { O/W exceptional financing } \\
\text { PV of external debt } 4 /\end{array}$ & $\begin{array}{l}-6.0 \\
92.6\end{array}$ & & & $\begin{array}{r}-1.3 \\
110.4\end{array}$ & $\begin{array}{r}-35.6 \\
150.5\end{array}$ & $\begin{array}{r}-4.3 \\
160.4\end{array}$ & $\begin{array}{r}-3.4 \\
163.1\end{array}$ & 155.6 & 142.8 & & 49.7 & $\begin{array}{r}-0.4 \\
32.7\end{array}$ & \\
\hline In percent of exports & $\begin{array}{r}92.6 \\
150.4\end{array}$ & & & 271.7 & 359.6 & 334.3 & 328.5 & 297.1 & 256.9 & & 82.6 & 63.3 & \\
\hline PV of PPG external debt & 92.6 & & & 104.1 & 135.1 & 133.7 & 131.0 & 121.8 & 108.2 & & 46.9 & 32.7 & \\
\hline In percent of exports & 150.4 & & & 256.1 & 322.9 & 278.6 & 263.8 & 232.7 & 194.7 & & 78.0 & 63.4 & \\
\hline In percent of government revenues & 501.5 & & & 572.3 & 690.9 & 645.6 & 596.9 & 530.1 & 489.1 & & 202.1 & 133.5 & \\
\hline Debt service-to-exports ratio (in percent) & 3.6 & & & 4.1 & 11.3 & 13.5 & 11.4 & 12.8 & 19.4 & & 12.4 & 4.6 & \\
\hline PPG debt service-to-exports ratio (in percent) & 3.6 & & & 4.1 & 11.3 & 13.5 & 11.4 & 9.3 & 15.3 & & 4.6 & 4.6 & \\
\hline PPG debt service-to-revenue ratio (in percent) & 11.9 & & & 9.2 & 24.1 & 31.4 & 25.8 & 21.1 & 38.4 & & 12.0 & 9.8 & \\
\hline Total gross financing need (Billions of U.S. dollars) & 0.0 & & & 1.1 & 2.4 & 2.8 & 2.3 & 2.3 & 2.6 & & 1.1 & 3.2 & \\
\hline Non-interest current account deficit that stabilizes debt ratio & 8.9 & & & 3.4 & 5.8 & 11.6 & 13.1 & 19.2 & 17.6 & & 15.5 & 8.9 & \\
\hline \multicolumn{14}{|l|}{ Key macroeconomic assumptions } \\
\hline Real GDP growth (in percent) & 6.2 & 2.8 & 5.3 & 2.7 & 5.4 & 7.2 & 6.9 & 8.2 & 6.8 & 6.2 & 4.8 & 4.4 & 4.9 \\
\hline GDP deflator in US dollar terms (change in percent) & 9.4 & 2.3 & 13.8 & -7.3 & 4.8 & 1.5 & 1.5 & 1.6 & 1.6 & 0.6 & 2.2 & 2.2 & 2.1 \\
\hline Effective interest rate (percent) 5/ & 0.3 & 0.3 & 0.1 & 0.2 & 1.4 & 2.3 & 1.9 & 1.7 & 1.6 & 1.5 & 1.3 & 0.9 & 1.1 \\
\hline Growth of exports of G\&S (US dollar terms, in percent) & 8.7 & 22.0 & 31.7 & -37.2 & 13.8 & 24.7 & 12.3 & 15.9 & 15.3 & 7.5 & 7.0 & 4.7 & 6.7 \\
\hline Growth of imports of G\&S (US dollar terms, in percent) & 28.8 & 25.2 & 31.8 & -19.7 & 19.6 & 23.9 & 1.4 & 5.1 & 6.9 & 6.2 & 6.6 & 4.8 & 5.8 \\
\hline Grant element of new public sector borrowing (in percent) & & $\ldots$ & $\ldots$ & 21.6 & 28.4 & 27.5 & 32.6 & 34.2 & 37.6 & 30.3 & 42.9 & 41.2 & 42.5 \\
\hline Government revenues (excluding grants, in percent of GDP) & 18.5 & & & 18.2 & 19.6 & 20.7 & 21.9 & 23.0 & 22.1 & & 23.2 & 24.5 & 23.5 \\
\hline Aid flows (in Billions of US dollars) 6/ & 0.4 & & & 1.6 & 1.5 & 1.8 & 1.5 & 1.6 & 1.7 & & 2.0 & & \\
\hline o/w Grants & 0.2 & & & 0.9 & 0.8 & 0.9 & 0.9 & 0.8 & 1.0 & & 1.0 & 0.8 & \\
\hline o/w Concessional loans & 0.1 & & & 0.8 & 0.7 & 0.9 & 0.6 & 0.7 & 0.7 & & 1.0 & & \\
\hline Grant-equivalent financing (in percent of GDP) 7/ & & & & 9.4 & 20.5 & 11.1 & 10.6 & 8.5 & 9.1 & & 6.3 & 3.1 & 5.5 \\
\hline Grant-equivalent financing (in percent of external financing) $7 /$ & $\cdots$ & & & 64.1 & 37.1 & 49.6 & 54.0 & 57.4 & 63.3 & & 68.1 & 60.6 & 67.0 \\
\hline \multicolumn{14}{|l|}{ Memoran } \\
\hline Nominal GDP (Billions of US dollars) & 11.6 & & & 11.0 & 12.2 & 13.3 & 14.4 & 15.8 & 17.2 & & 25.1 & 48.3 & \\
\hline Nominal dollar GDP growth & 16.1 & & & -4.8 & 10.4 & 8.8 & 8.5 & 9.9 & 8.6 & 6.9 & 7.1 & 6.7 & 7.1 \\
\hline PV of PPG external debt (in Billions of US dollars) & 10.7 & & & 11.5 & 16.5 & 17.7 & 18.8 & 19.3 & 18.6 & & 11.8 & 15.8 & \\
\hline (PVt-PVt-1)/GDPt-1 (in percent) & & & & 6.4 & 45.2 & 10.3 & 8.5 & 2.9 & -4.3 & 11.5 & -3.8 & 0.9 & -1.4 \\
\hline
\end{tabular}

Source: IMF staff simulations.

1/ Includes both public and private sector external debt.

$3 /$ Includes exceptional financing (i.e., changes in arrears and debt relief); changes in gross foreign assets; and valuation adjustments. For projections also includes contribution from price and exchange rate changes. 4/ Assumes that PV of private sector debt is equivalent to its face value.

6/ Current-year interest payments divided by previous peris as grants, concessional loans, and debt relief.

7/ Grant-equivalent financing includes grants provided directly to the government and through new borrowing (difference between the face value and the PV of new debt). 
Table 2. Democratic Republic of the Congo: Sensitivity Analysis for Key Indicators of Public and Publicly Guaranteed External Debt, 2009-29 (In percent)

\begin{tabular}{|c|c|c|c|c|c|c|c|c|c|c|c|c|}
\hline & \multicolumn{12}{|c|}{ Projections } \\
\hline & 2009 & 2010 & 2011 & 2012 & 2013 & 2014 & 2015 & 2016 & 2017 & 2018 & 2019 & 2029 \\
\hline \multicolumn{13}{|c|}{ PV of debt-to GDP ratio } \\
\hline Baseline & 104.1 & 135.1 & 133.7 & 131.0 & 121.8 & 108.2 & 94.4 & 82.1 & 68.7 & 54.0 & 46.9 & 32.7 \\
\hline \multicolumn{13}{|l|}{ A. Alternative Scenarios } \\
\hline A1. Key variables at their historical averages in 2009-2029 1/ & 104.1 & 129.0 & 119.2 & 111.0 & 99.8 & 83.6 & 72.6 & 64.9 & 54.7 & 40.8 & 36.1 & 3.5 \\
\hline A2. New public sector loans on less favorable terms in 2009-2029 2/ & 104.1 & 148.7 & 151.3 & 154.0 & 150.4 & 139.9 & 127.9 & 115.5 & 105.6 & 97.1 & 94.8 & 75.3 \\
\hline \multicolumn{13}{|l|}{ B. Bound Tests } \\
\hline B1. Real GDP growth at historical average minus one standard deviation in 2010-2011 & 104.1 & 145.9 & 158.4 & 155.1 & 144.2 & 128.1 & 111.6 & 97.0 & 81.2 & 63.7 & 55.3 & 38.6 \\
\hline B2. Export value growth at historical average minus one standard deviation in 2010-2011 3/ & 104.1 & 141.2 & 153.6 & 149.9 & 139.6 & 125.1 & 110.3 & 97.2 & 83.3 & 68.0 & 60.2 & 37.0 \\
\hline B3. US dollar GDP deflator at historical average minus one standard deviation in 2010-2011 & 104.1 & 160.0 & 181.4 & 177.7 & 165.2 & 146.7 & 127.8 & 111.1 & 93.0 & 73.0 & 63.3 & 44.2 \\
\hline $\begin{array}{l}\text { B4. Net non-debt creating flows at historical average minus one standard deviation in 2010-2011 } 4 /\end{array}$ & 104.1 & 143.5 & 150.9 & 147.3 & 137.1 & 122.8 & 108.1 & 95.1 & 81.2 & 66.1 & 58.2 & 36.3 \\
\hline B5. Combination of B1-B4 using one-half standard deviation shocks & 104.1 & 166.1 & 206.5 & 201.4 & 187.6 & 168.3 & 148.7 & 131.3 & 112.8 & 92.7 & 82.2 & 49.6 \\
\hline B6. One-time 30 percent nominal depreciation relative to the baseline in $20105 /$ & 104.1 & 189.3 & 187.0 & 183.2 & 170.3 & 151.2 & 131.8 & 114.6 & 95.8 & 75.2 & 65.3 & 45.6 \\
\hline \multicolumn{13}{|c|}{ PV of debt-to-exports ratio } \\
\hline Baseline & 256.1 & 322.9 & 278.6 & 263.8 & 232.7 & 194.7 & 163.0 & 137.4 & 114.2 & 89.7 & 78.0 & 63.4 \\
\hline \multicolumn{13}{|l|}{ A. Alternative Scenarios } \\
\hline A1. Key variables at their historical averages in 2009-2029 1/ & 256.1 & 308.3 & 248.4 & 223.5 & 190.5 & 150.4 & 125.4 & 108.5 & 90.8 & 67.7 & 60.0 & 6.8 \\
\hline A2. New public sector loans on less favorable terms in 2009-2029 $2 /$ & 256.1 & 355.4 & 315.5 & 310.1 & 287.2 & 251.7 & 221.0 & 193.2 & 175.3 & 161.3 & 157.7 & 145.7 \\
\hline \multicolumn{13}{|l|}{ B. Bound Tests } \\
\hline B1. Real GDP growth at historical average minus one standard deviation in 2010-2011 & 256.1 & 322.8 & 278.3 & 263.4 & 232.2 & 194.2 & 162.5 & 136.8 & 113.6 & 89.2 & 77.5 & 63.0 \\
\hline B2. Export value growth at historical average minus one standard deviation in 2010-2011 3/ & 256.1 & 424.9 & 556.8 & 525.0 & 463.6 & 391.3 & 331.5 & 282.9 & 240.5 & 196.5 & 174.1 & 124.6 \\
\hline B3. US dollar GDP deflator at historical average minus one standard deviation in 2010-2011 & 256.1 & 322.8 & 278.3 & 263.4 & 232.2 & 194.2 & 162.5 & 136.8 & 113.6 & 89.2 & 77.5 & 63.0 \\
\hline B4. Net non-debt creating flows at historical average minus one standard deviation in 2010-2011 4/ & 256.1 & 342.9 & 314.5 & 296.7 & 261.9 & 220.9 & 186.8 & 159.2 & 134.9 & 109.8 & 96.8 & 70.4 \\
\hline B5. Combination of B1-B4 using one-half standard deviation shocks & 256.1 & 368.2 & 412.1 & 388.3 & 343.0 & 289.9 & 246.0 & 210.4 & 179.4 & 147.4 & 130.7 & 92.0 \\
\hline B6. One-time 30 percent nominal depreciation relative to the baseline in 20105 / & 256.1 & 322.8 & 278.3 & 263.4 & 232.2 & 194.2 & 162.5 & 136.8 & 113.6 & 89.2 & 77.5 & 63.0 \\
\hline \multicolumn{13}{|c|}{ PV of debt-to-revenue ratio } \\
\hline Baseline & 572.3 & 690.9 & 645.6 & 596.9 & 530.1 & 489.1 & 418.0 & 362.2 & 300.4 & 233.9 & 202.1 & 133.5 \\
\hline \multicolumn{13}{|l|}{ A. Alternative Scenarios } \\
\hline A1. Key variables at their historical averages in 2009-2029 1/ & 572.3 & 659.6 & 575.7 & 505.8 & 434.0 & 377.6 & 321.6 & 286.2 & 238.8 & 176.4 & 155.4 & 14.3 \\
\hline A2. New public sector loans on less favorable terms in 2009-2029 $2 /$ & 572.3 & 760.4 & 731.0 & 701.6 & 654.2 & 632.1 & 566.6 & 509.6 & 461.3 & 420.2 & 408.4 & 307.0 \\
\hline \multicolumn{13}{|l|}{ B. Bound Tests } \\
\hline B1. Real GDP growth at historical average minus one standard deviation in 2010-2011 & 572.3 & 745.8 & 765.1 & 706.9 & 627.5 & 578.7 & 494.2 & 428.1 & 354.7 & 275.7 & 238.0 & 157.4 \\
\hline B2. Export value growth at historical average minus one standard deviation in 2010-2011 3/ & 572.3 & 721.7 & 742.0 & 683.1 & 607.3 & 565.2 & 488.7 & 429.0 & 363.8 & 294.5 & 259.3 & 151.0 \\
\hline B3. US dollar GDP deflator at historical average minus one standard deviation in 2010-2011 & 572.3 & 818.3 & 876.2 & 809.6 & 718.7 & 662.8 & 566.0 & 490.3 & 406.2 & 315.8 & 272.6 & 180.2 \\
\hline B4. Net non-debt creating flows at historical average minus one standard deviation in 2010-2011 4/ & 572.3 & 733.6 & 728.8 & 671.3 & 596.6 & 554.7 & 478.9 & 419.8 & 355.0 & 286.1 & 250.8 & 148.2 \\
\hline B5. Combination of B1-B4 using one-half standard deviation shocks & 572.3 & 849.4 & 997.4 & 917.8 & 816.1 & 760.6 & 658.8 & 579.4 & 492.9 & 401.1 & 353.7 & 202.5 \\
\hline B6. One-time 30 percent nominal depreciation relative to the baseline in 20105 / & 572.3 & 967.7 & 903.5 & 834.9 & 741.1 & 683.4 & 583.7 & 505.6 & 418.8 & 325.6 & 281.1 & 185.8 \\
\hline \multicolumn{13}{|c|}{ Debt service-to-exports ratio } \\
\hline Baseline & 4.1 & 11.3 & 13.5 & 11.4 & 9.3 & 15.3 & 13.3 & 11.7 & 10.9 & 10.4 & 4.6 & 4.6 \\
\hline \multicolumn{13}{|l|}{ A. Alternative Scenarios } \\
\hline A1. Key variables at their historical averages in 2009-2029 1/ & 4.1 & 11.8 & 14.1 & 11.7 & 9.4 & 16.9 & 15.0 & 13.6 & 13.1 & 12.8 & 4.3 & 3.4 \\
\hline A2. New public sector loans on less favorable terms in 2009-2029 $2 /$ & 4.1 & 11.3 & 17.7 & 16.6 & 15.1 & 20.6 & 21.5 & 18.9 & 17.4 & 17.8 & 16.4 & 8.8 \\
\hline \multicolumn{13}{|l|}{ B. Bound Tests } \\
\hline B1. Real GDP growth at historical average minus one standard deviation in 2010-2011 & 4.1 & 11.3 & 13.5 & 11.4 & 9.3 & 15.3 & 13.3 & 11.7 & 10.9 & 10.4 & 4.6 & 4.6 \\
\hline B2. Export value growth at historical average minus one standard deviation in 2010-2011 3/ & 4.1 & 14.2 & 24.0 & 21.2 & 17.4 & 27.7 & 24.1 & 21.2 & 19.8 & 18.8 & 9.5 & 10.0 \\
\hline B3. US dollar GDP deflator at historical average minus one standard deviation in 2010-2011 & 4.1 & 11.3 & 13.5 & 11.4 & 9.3 & 15.3 & 13.3 & 11.7 & 10.9 & 10.4 & 4.6 & 4.6 \\
\hline B4. Net non-debt creating flows at historical average minus one standard deviation in 2010-2011 4/ & 4.1 & 11.3 & 13.9 & 12.1 & 9.9 & 15.8 & 13.8 & 12.1 & 11.3 & 10.8 & 5.5 & 5.6 \\
\hline B5. Combination of B1-B4 using one-half standard deviation shocks & 4.1 & 12.1 & 17.5 & 15.6 & 12.7 & 20.2 & 17.6 & 15.4 & 14.4 & 13.7 & 7.2 & 7.5 \\
\hline B6. One-time 30 percent nominal depreciation relative to the baseline & 4.1 & 11.3 & 13.5 & 11.4 & 9.3 & 15.3 & 13.3 & 11.7 & 10.9 & 10.4 & 4.6 & 4.6 \\
\hline
\end{tabular}

Debt service-to-revenue ratio

Baseline

A. Alternative Scenarios

A1. Key variables at their historical averages in 2009-2029 $1 /$

A2. New public sector loans on less favorable terms in 2009-2029 21

B. Bound Tests

B1. Real GDP growth at historical average minus one standard deviation in 2010-2011

B2. Export value growh at historical average minus one standard deviation in 2010-2011 $3 /$

B4. Net non-debt creating flows at historical average minus one standard deviation in 2010-2011 4/

B5. Combination of B1-B4 using one-half standard deviation shocks

B6. One-time 30 percent nominal depreciation relative to the baseline in $20105 /$

Memorandum item:

Grant element assumed on residual financing (i.e., financing required above baseline) $6 /$

Source: IMF staff projections and simulations.

1/ Variables include real GDP growth, growth of GDP deflator (in U.S. dollar terms), non-interest current account in percent of GDP, and non-debt creating flows.

2/ Assumes that the interest rate on new borrowing is by 2 percentage points higher than in the baseline., while grace and maturity periods are the same as in the baseline.

of GDP is assumed to return to its baseline level after the shock (implicitly assuming an offsetting adjustment

$4 /$ Includes official and private transfers and FDI.

$5 /$ Depreciation is defined as percentage decline in dollar/local currency rate, such that it never exceeds 100 percent.

6/ Applies to all stress scenarios except for A2 (less favorable financing) in which the terms on all new financing are as specified in footnote 2.

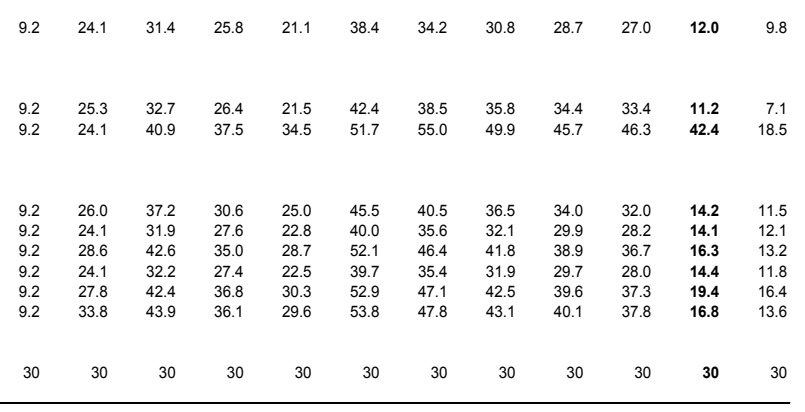

30 
Table 3. Democratic Republic of the Congo: Public Sector Debt Sustainability Framework, Baseline Scenario, 2008-29 (In percent of GDP, unless otherwise indicated)

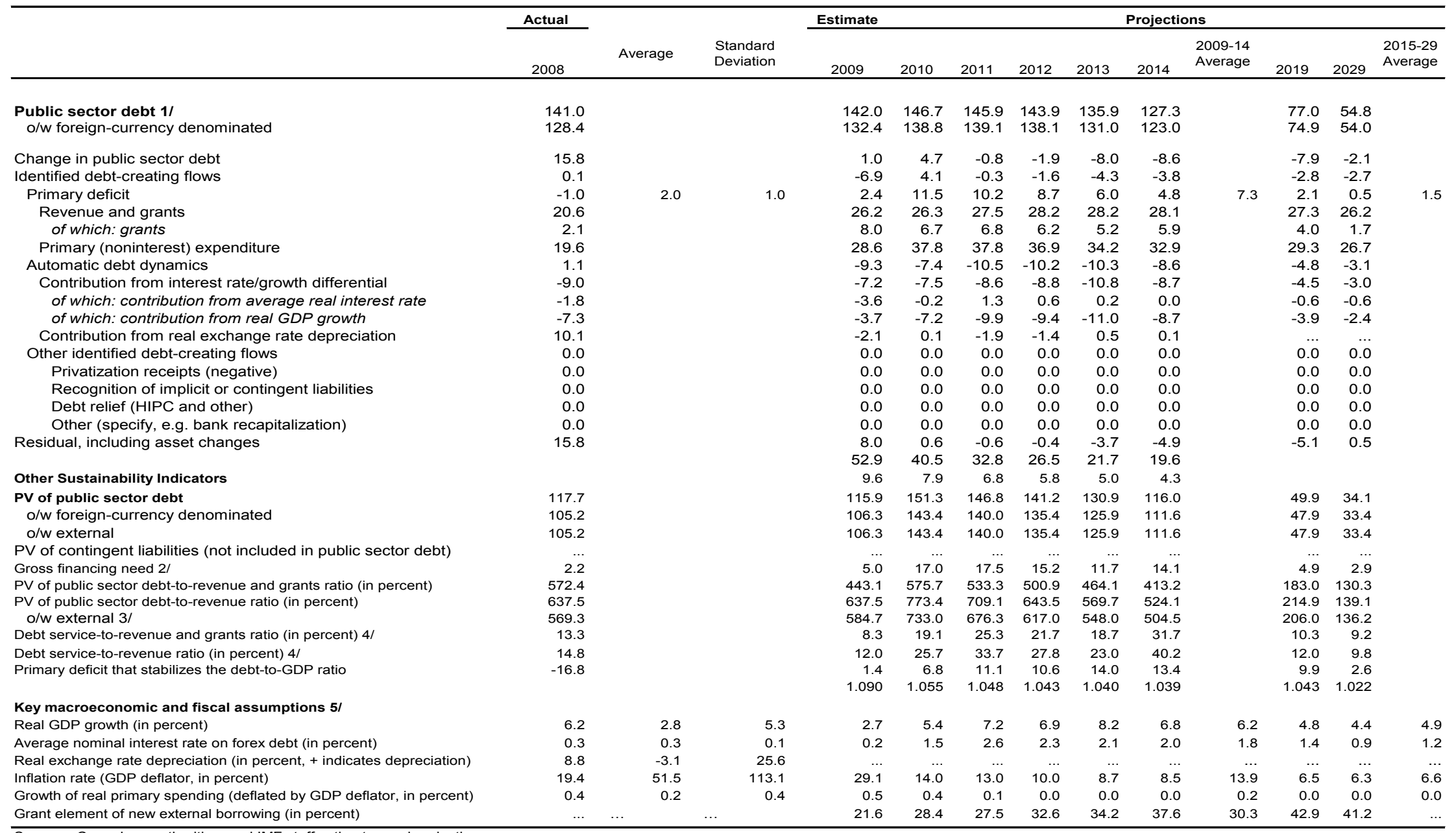

Sources: Congolese authorities; and IMF staff estimates and projections.

1/ Gross general government debt.

/ Gross financing need is defined as the primary deficit plus debt service plus the stock of short-term debt at the end of the last period.

3/ Revenues excluding grants.

5/ Historical averages and standard deviations are generally derived over the past 10 years, subject to data availability. 
Table 4. Democratic Republic of the Congo: Sensitivity Analysis for Key Indicators of Public Debt 2009-29

\begin{tabular}{|c|c|c|c|c|c|c|c|c|}
\hline & \multicolumn{8}{|c|}{ Projections } \\
\hline & 2009 & 2010 & 2011 & 2012 & 2013 & 2014 & 2019 & 2029 \\
\hline \multicolumn{9}{|l|}{ PV of Debt-to-GDP Ratio } \\
\hline Baseline & 115.9 & 151.3 & 146.8 & 141.2 & 130.9 & 116.0 & 49.9 & 34.1 \\
\hline \multicolumn{9}{|l|}{ A. Alternative scenarios } \\
\hline A1. Real GDP growth and primary balance are at historical averages & 115.9 & 145.4 & 138.4 & 130.8 & 121.5 & 105.0 & 25.9 & 9.4 \\
\hline A2. Primary balance is unchanged from 2009 & 115.9 & 143.2 & 132.1 & 121.6 & 109.0 & 92.7 & 30.9 & 30.0 \\
\hline A3. Permanently lower GDP growth $1 /$ & 115.9 & 153.1 & 150.6 & 147.1 & 138.8 & 125.5 & 67.5 & 91.2 \\
\hline \multicolumn{9}{|l|}{ B. Bound tests } \\
\hline B1. Real GDP growth is at historical average minus one standard deviations in $2010-2011$ & 115.9 & 164.7 & 179.0 & 175.9 & 167.1 & 152.6 & 90.1 & 101.5 \\
\hline B2. Primary balance is at historical average minus one standard deviations in $2010-2011$ & 115.9 & 143.7 & 133.1 & 128.2 & 118.4 & 103.9 & 39.6 & 26.1 \\
\hline B3. Combination of B1-B2 using one half standard deviation shocks & 115.9 & 150.1 & 147.5 & 144.4 & 136.0 & 122.1 & 61.0 & 67.0 \\
\hline B4. One-time 30 percent real depreciation in 2010 & 115.9 & 219.0 & 208.5 & 197.8 & 183.7 & 163.3 & 81.3 & 62.2 \\
\hline B5. 10 percent of GDP increase in other debt-creating flows in 2010 & 115.9 & 160.2 & 155.3 & 149.4 & 138.7 & 123.5 & 56.4 & 39.1 \\
\hline
\end{tabular}

PV of Debt-to-Revenue Ratio 2/

Baseline

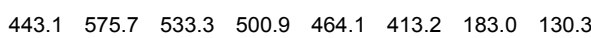

\section{A. Alternative scenarios}

A1. Real GDP growth and primary balance are at historical averages

A2. Primary balance is unchanged from 2009

A3. Permanently lower GDP growth 1/

\section{B. Bound tests}

B1. Real GDP growth is at historical average minus one standard deviations in 2010-2011 B2. Primary balance is at historical average minus one standard deviations in 2010-2011

B3. Combination of B1-B2 using one half standard deviation shocks

B4. One-time 30 percent real depreciation in 2010

B5. 10 percent of GDP increase in other debt-creating flows in 2010

Debt Service-to-Revenue Ratio 2/

Baseline

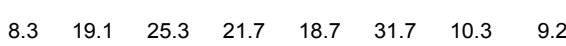

A. Alternative scenarios

A1. Real GDP growth and primary balance are at historical averages

A2. Primary balance is unchanged from 2009

A3. Permanently lower GDP growth 1/

$\begin{array}{llllllll}443.1 & 549.9 & 494.4 & 452.8 & 417.5 & 357.7 & 89.8 & 34.5\end{array}$ $\begin{array}{llllllll}443.1 & 544.9 & 479.9 & 431.2 & 386.2 & 330.2 & 113.4 & 114.7 \\ 443.1 & 581.1 & 544.2 & 518.0 & 487.9 & 442.1 & 243.3 & 343.0\end{array}$

$\begin{array}{llllllll}443.1 & 614.3 & 621.7 & 599.0 & 572.5 & 523.1 & 321.7 & 383.0\end{array}$ $\begin{array}{llllllll}443.1 & 546.9 & 483.6 & 454.5 & 419.8 & 370.3 & 145.1 & 99.7\end{array}$ $\begin{array}{llllllll}443.1 & 563.7 & 519.6 & 498.4 & 471.3 & 423.7 & 219.8 & 253.9\end{array}$ $\begin{array}{lllllllll}443.1 & 833.3 & 757.7 & 701.6 & 651.0 & 581.7 & 298.2 & 237.5\end{array}$ $\begin{array}{lllllllllllll}443.1 & 609.5 & 564.2 & 529.8 & 491.7 & 440.0 & 206.7 & 1 & & \end{array}$

$\begin{array}{rrrrrrrr}8.3 & 19.5 & 25.1 & 20.4 & 17.0 & 31.9 & 7.3 & -2.6 \\ 8.3 & 19.1 & 24.1 & 19.2 & 15.6 & 28.3 & 7.3 & 4.3 \\ 8.3 & 19.3 & 25.8 & 22.3 & 19.6 & 33.4 & 12.8 & 20.4\end{array}$

\section{B. Bound tests}

B1. Real GDP growth is at historical average minus one standard deviations in 2010-2011 B2. Primary balance is at historical average minus one standard deviations in 2010-2011 B3. Combination of B1-B2 using one half standard deviation shocks B4. One-time 30 percent real depreciation in 2010

B5. 10 percent of GDP increase in other debt-creating flows in 2010

Debt Service-to-GDP Ratio

Baseline

$\begin{array}{rrrrrrrr}8.3 & 20.2 & 28.9 & 25.4 & 22.7 & 38.0 & 16.1 & 25.5 \\ 8.3 & 19.1 & 24.1 & 19.4 & 16.6 & 29.8 & 8.7 & 5.7 \\ 8.3 & 19.8 & 26.2 & 20.9 & 18.3 & 33.2 & 11.8 & 14.9 \\ 8.3 & 24.0 & 37.6 & 32.6 & 28.6 & 48.6 & 19.1 & 23.2 \\ 8.3 & 19.1 & 26.7 & 23.2 & 19.9 & 32.9 & 11.2 & 11.3\end{array}$

$\begin{array}{llllllll}2.2 & 5.0 & 7.0 & 6.1 & 5.3 & 8.9 & 2.8 & 2.4\end{array}$

A. Alternative scenarios

A1. Real GDP growth and primary balance are at historical averages A2. Primary balance is unchanged from 2006 A3. Permanently lower GDP growth 1/

B. Bound tests

B1. Real GDP growth is at historical average minus one standard deviations in 2008-2009 B2. Primary balance is at historical average minus one standard deviations in 2008-2009

B3. Combination of B1-B2 using one half standard deviation shocks

B4. One-time 30 percent real depreciation in 2008

B5. 10 percent of GDP increase in other debt-creating flows in 2008

$\begin{array}{rrrrrrrr}2.2 & 5.4 & 8.3 & 7.5 & 6.6 & 11.1 & 4.5 & 6.7 \\ 2.2 & 5.0 & 6.6 & 5.5 & 4.7 & 8.4 & 2.4 & 1.5 \\ 2.2 & 5.3 & 7.4 & 6.0 & 5.3 & 9.6 & 3.3 & 3.9 \\ 2.2 & 6.3 & 10.4 & 9.2 & 8.1 & 13.6 & 5.2 & 6.1 \\ 2.2 & 5.0 & 7.4 & 6.5 & 5.6 & 9.2 & 3.1 & 3.0\end{array}$

Sources: Congolese authorities; and IMF staff estimates and projections.

1/ Assumes that real GDP growth is at baseline minus one standard deviation divided by the square root of the length of the projection period. 2/ Revenues are defined inclusive of grants.

\section{CInternational Monetary Fund. Not for Redistribution}


INTERNATIONAL MONETARY FUND AND

INTERNATIONAL DEVELOPMENT ASSOCIATION

DEMOCRATIC REPUBLIC OF CONGO

\begin{abstract}
Joint IMF/World Bank Debt Sustainability Analysis 2009
Prepared by the Staffs of the International Monetary Fund and the International Development Association

Approved by Mark Plant and Dominique Desruelle (IMF) and Carlos Alberto Braga and Sudhir Shetty (IDA)
\end{abstract}

November 30, 2009

This note provides an updated debt sustainability analysis (DSA) for the Democratic Republic of the Congo (DRC) using the joint Bank-Fund Debt Sustainability Framework (DSF) for Low Income Countries. The results indicate that the DRC remains in debt distress, as was the case in the most recent DSA conducted in August 2007. In the absence of debt relief provided under the enhanced Heavily Indebted Poor Country (HIPC) Initiative and Multilateral Debt Relief Initiative (MDRI), external debt burden indicators are projected to remain above policy-dependent thresholds over prolonged periods. Even after HIPC/MDRI debt relief, the external debt outlook would be vulnerable to adverse shocks. External borrowing to finance public infrastructure projects under the Sino-Congolese Cooperation Agreement (SCCA) are projected to increase significantly the country's external debt burden indicators over the medium term, but related risks to debt sustainability appear manageable.

35. The Democratic Republic of the Congo (DRC) remains in debt distress. At end 2008 the present value of public and publicly guaranteed (PPG) external debt is estimated at 93 percent of GDP, 150 percent of exports and 501 percent of government revenue (excluding grants), well above policy-dependent threshold levels. ${ }^{17}$ Moreover, all debt burden indicators are projected to worsen significantly in 2009 due to marked declines in GDP, exports and government revenue in the wake of the global economic slowdown and accompanying sharp decline in world commodity prices.

\footnotetext{
${ }^{17}$ With an average CPIA rating of 2.80 in $2006-08$, the DRC is classified having a weak policy framework.
} 


\section{BACKGROUND}

36. The DRC continues to accumulate external arrears mainly on debt owed to bilateral creditors. The DRC reached the decision point of the enhanced HIPC Initiative in July 2003, qualifying for interim debt relief. ${ }^{18}$ The IMF discontinued interim assistance in June 2006 when the PRGF program went off track. The Paris Club agreed to reschedule DRC's debt under Cologne flow terms in November 2003, which was also suspended in June 2006 when the PRGF program went off track. The International Development Agency's (IDA) interim HIPC assistance expired in October 2009. The African Development Bank/Fund will deliver interim assistance in 2010. The nominal value of PPG external debt (including arrears) is estimated at US\$13.1 billion at end-2008, US\$7.1 billion of which is owed to bilateral creditors, US\$4.4 billion to multilateral institutions, and US\$1.5 billion to commercial creditors. Arrears are estimated at US\$3.3 billion at end-2008, of which US\$1.5 billion is owed to Paris Club creditors.

\section{External DebT Sustainability Analysis}

37. The baseline scenario in this DSA assumes a strong recovery in economic activity over the medium term, supported by large investments in mining and public infrastructure projects. In particular, it assumes that the security situation in the eastern provinces stabilizes and that the government adopts prudent macroeconomic policies and makes significant progress on key structural reforms, including strengthening tax collection, improving budget preparation and execution, and reforming the civil service. The near-term outlook is dominated by the impact of the global economic slowdown (Box 1), followed by a strong recovery over the medium term fueled by a US\$3.2 billion investment in a mining project along with US\$3 billion in investments in public infrastructure projects envisaged under the SCCA (Box 2).

38. The baseline scenario assumes that a new three-year PRGF arrangement will be in place by the end of 2009, which will allow the IMF and the DRC's Paris Club creditors to provide additional interim assistance under the enhanced HIPC Initiative. ${ }^{19}$ It also assumes that arrears to bilateral creditors are rescheduled or deferred, the African Development Bank/Fund delivers interim HIPC assistance in 2010, and that IDA provided interim HIPC assistance up to October 2009.

\footnotetext{
18 "Democratic Republic of the Congo-Decision Point Document for the Enhanced Heavily Indebted Poor Countries (HIPC) Initiative", and IDA/R2003-0059.

${ }^{19}$ The baseline scenario does not assume that the DRC reaches the HIPC completion point in accordance with current LIC DSA guidelines outlined in: "Staff Guidance Note on the Application of the Joint Fund-Bank Debt Sustainability Framework for Low-Income Countries”, April 2007and IDA/No. 39748. An alternative scenario assumes that the DRC reaches the HIPC completion point in mid-2010.
} 


\section{Box 1. Democratic Republic of the Congo: Macroeconomic Assumptions for the DSA}

Although short-term economic conditions remain weak, the medium-term outlook is positive. The baseline projection scenario has the following key elements.

- GDP growth declines from 6.2 percent in 2008 to 2.7 percent in 2009 due to the slowdown in global growth and the sharp decline in world commodity prices from record levels reached in mid-2008. Growth rebounds strongly over the medium term, averaging 7.5 percent over the period 2010-16, spurred by large investment expenditures under the SCCA (Box 2), and a recovery in mining activity. The mining project financed by the SCCA is projected to increase real GDP growth by 0.3 percentage points on average during the construction phase 2009-12 and by 0.6 percentage points on average during the production phase in 2013-16. The public infrastructure projects financed by SCCA are projected to increase real GDP growth by 0.7 percentage points on average during the construction phase 2009-12, declining to 0.2 percentage points in the long run (Box 3).

- Inflation declines to 48.7 percent by end-2009 and to single digits over the medium term, supported by prudent fiscal and monetary policies.

- The overall fiscal deficit (including grants) widens substantially in the short run, reaching 12 percent in 2010, before narrowing gradually to around 2 percent of GDP in the long run as ongoing reforms expand the tax base and noninterest expenditures decline slowly as a percent of GDP. Grants decline from 8.7 percent of GDP in 2009 to under 4 percent in 2018.

- The current account deficit widens from 15 percent of GDP in 2008 to 32 percent in 2011 mainly due to a large increase in imported factors used in the mining and public infrastructure projects. The deficit narrows thereafter as mining exports expand and investment-related imports diminish, stabilizing at around 6 percent of GDP over the long run.

- $\quad$ External financing over the medium term is dominated by loans disbursed to fund public infrastructure projects under the SCCA, accounting for 22 percent of gross borrowing needs over the period 2009-14. The average grant element of new borrowing rises steadily from 20 percent in 2009 to 43 percent in 2015 and beyond.

- $\quad$ Net foreign direct investment declines slightly from an average level of 6.3 percent of GDP in 2004-08 to 5.8 percent in 2009 in the wake of the global economic downturn, recovering to an average level of 7.2 percent of GDP by 2010-12 led by foreign investment in mining projects. 


\section{Box 2. Democratic Republic of the Congo: The Sino-Congolese Cooperation Agreement (SCCA)}

In April 2008, the DRC signed a cooperation agreement between a Congolese parastatal mining enterprise (GECAMINES) and a consortium of Chinese enterprises, forming a joint venture company (SICOMINES). The net operating income from the joint venture is to be distributed by equity shares with 32 percent going to GECAMINES and 68 percent to the consortium of Chinese enterprises.

The original agreement (signed in April 2008) included a US\$3.2 billion mining project, along with US\$6 billion in public infrastructure projects to be implemented in two phases over the period 2009-17. The agreement was amended in October 2009 to exclude the second phase of public infrastructure projects totaling US $\$ 3$ billion that was to be implemented over the period 2014-17, leaving just the first phase of public infrastructure projects totaling US\$3 billion to be implemented over the period 2009-13.

Under the original agreement, the Congolese government provided a public guarantee on the loans for the mining and public infrastructure projects. However, the public guarantee on the mining loan was removed in the amended agreement. The public guarantee requires that the Congolese government repay any principal outstanding on the public infrastructure loans at end 2034 (25 years after the formation of the joint venture SICOMINES) with capitalized interest.

The US $\$ 3.2$ billion mining investment is to be financed by a US $\$ 1.1$ billion interest-free loan along with a US\$2.1 billion loan with an interest rate of 6.1 percent. The US\$3 billion investment in public infrastructure is to be financed by a series of loans to be disbursed for the individual projects, each with a fixed interest rate of 4.4 percent (LIBOR plus 100 basis points as of April 22, 2008). All loans are denominated in US dollars.

The consortium of Chinese enterprises provides the DRC government with a US\$250 million signing bonus ("pas de porte"), half of which was disbursed in March 2009. The remaining balance will be disbursed upon the transfer of the mining concession title to SICOMINES. GECAMINES receives a US\$100 million signing bonus, also disbursed in two tranches.

The mining project is expected to generate operating profits beginning in 2013, which will be used to repay the mining and public infrastructure loans in three stages. In the first stage, all profits from the mine will go to repay principal and capitalized interest on US\$375 million in "urgent" public infrastructure loans. In the second stage, 85 percent of operating profits will be used to repay the mining loans. In the third stage, 85 percent of operating profits will be used to repay the remaining public infrastructure loans. In the second and third stages, 15 percent of operating profits will be paid to the shareholders of the joint venture SICOMINES. 


\section{Box 3. The Democratic Republic of the Congo: The Economic Impact of Public Infrastructure Projects}

A two-stage approach was used to analyze the long-run growth impact of the US\$3 billion in public infrastructure projects to be undertaken under the SCCA. ${ }^{1}$ In the first stage, the analysis identified the location and size of current economic activities (e.g., agriculture, forestry, and mining) and the locationspecific economic potential. Location-specific transport costs take the distance to relevant markets into account using a "cost-distance" algorithm. Sector-specific responses to changes in transport costs were estimated and used to simulate the long-term impact of transport investments on production location levels. Long-term elasticities based on cross-sectional reduced-form regressions were used to estimate the response of local production to a change in transportation costs. In a second stage, a multiplier approach was used to gauge the demand side effects of public infrastructure projects during the implementation phase. The analysis indicates that the public infrastructure projects would raise real GDP growth by 0.7 percentage points on average during the construction phase 2009-13 mainly through the impact of the higher investment on domestic demand. The growth impact declines to 0.2 percentage points upon completion of the projects reflecting gains in total factor productivity associated with the improved public infrastructure.

See World Bank (2009, forthcoming), "Prioritizing Infrastructure Investments: A Spatial Approach”.

\section{Under the baseline scenario, external debt burden indicators breach} policy-dependent thresholds over prolonged periods (Figure 1). The present value of PPG external debt exceeds the 30 percent of GDP threshold over most of the 20 -year projection period (Figure 1b), notwithstanding the strong recovery projected over the medium term. The debt-to-export and debt-to-revenue ratio indicators decline somewhat faster over the projection period, falling below their thresholds in 2018 and 2019, respectively (Figures 1c and 1d). This reflects higher export revenues resulting from the large mining projects and higher tax revenue ensuing from reforms undertaken to broaden the tax base.

40. The baseline projections are subject to significant downside risks. Given the DRC's high dependence on mining exports, the external account and overall economic outlook remains highly vulnerable to adverse terms of trade shocks. Moreover, poor governance, unresolved security issues, weak implementation capacity and lower than envisaged donor support could also worsen the external debt outlook significantly.

41. All debt burden indicators worsen substantially under standard stress tests. The debt profile is most vulnerable to a shock that entails public sector debt financed under less favorable terms than assumed in the baseline (Figure 1). ${ }^{20}$ The external debt burden indicators decline somewhat more rapidly under the historical scenario. This reflects the fact

\footnotetext{
${ }^{20}$ The average grant element on net borrowing is only 30 percent in the period 2009-13, well below the 35 percent grant element required to qualify as concessional. The large difference reflects two main factors. First, the concessionality calculation is based on a much higher discount rate than that used in the DSA. Second, the DSA includes refinancing of arrears to bilateral creditors on non-concessional terms.
} 
that the country had no access to external financing during the conflict period which restricted the noninterest current account deficit.

\section{The external debt burden indicators are projected to breach their thresholds} even if the country were to receive debt relief under the enhanced HIPC Initiative and the MDRI. If the DRC were to reach the completion point in mid-2010, all three external debt burden indicators breach their thresholds (Figure 2). The external debt-to-GDP ratio breaches the 30 percent threshold over the period 2010-18 reaching a high of 45 percent in 2012 (Figure 2b). The external debt-to-revenue ratio breaches the 200 percent threshold slightly in just two years (2012 and 2014). New purchases under the proposed PRGF arrangement would account for 6.5 percent of the PV of external PPG debt in 2012 (2.9 percent of GDP), declining to 2.7 percent in 2019 ( 0.7 percent of GDP). The external debt profiles are robust to lower long-run growth projections. Reducing real GDP growth by half of a percentage point after 2017 (which roughly offsets the assumed benefit from the public infrastructure projects) raises the PV of the PPG external debt-to-GDP ratio by only 1.3 percentage points in 2029. The debt profiles are vulnerable to adverse shocks, however. In particular, two of the three external debt burden indicators breach their respective thresholds over the entire projection period under the most extreme stress test (Figures $2 \mathrm{~b}$ and $2 \mathrm{~d}$ ).

\section{The public guarantee on the infrastructure financing under the SCCA raises the} external debt burden indicators over the medium term but does not have a major impact on debt sustainability over the long term. Removing the public guarantee on the loans for the public infrastructure projects would reduce the external debt-to-GDP ratio by 8.6 percentage points on average over the period 2011-16, but would have no impact after 2019 when the loans are projected to be fully repaid (Figure 3). ${ }^{21}$ The present-value calculations underlying the DSA take into account that the public guarantee can only be invoked after 25 years. ${ }^{22}$ If the public guarantee were based on a conventional loan agreement with a fixed repayment schedule equal to the projected payments from the net operating income of SICOMINES, ${ }^{23}$ the external debt burden indicators would be just slightly higher over medium term, but with no impact beyond 2019 (Figure 3).

\footnotetext{
${ }^{21}$ The DSA includes the public guarantee on the infrastructure financing made under the SCCA as external PPG debt, but does not include the debt service payments made by the net operating profit from the joint venture SICOMINES.

${ }^{22}$ To illustrate, consider the following example. In 2009, the Chinese consortium is projected to disburse a total of $\$ 375$ million in loans for public infrastructure projects. The legal obligation of the government under the terms of the agreement is to repay any outstanding balance (principal and compounded interest) in 2034. In keeping with the conventional for valuing public guarantees, we assume the worst outcome-zero operating income over the entire 25-year period. This implies a legal obligation of $\$ 375$ million in principal and $\$ 725$ million in compounded interest in 2034, which has a present value of \$397 million in 2009 (based on a discount rate of 4 percent used in DSAs for low-income countries).

${ }^{23}$ For example, the $\$ 375$ million in loans to be disbursed in 2009 is projected to be fully repaid (principal and capitalized interest) in 2013. This would raise the present value of external debt by $\$ 426$ million in the case of a
} 


\section{Public Debt Sustainability analysis}

\section{The DRC's domestic debt is relatively low and as a result, the above external debt sustainability analysis broadly applies to total public debt, albeit with slightly} higher debt burden indicators (Figure 4). Although information on domestic debt is poor, the authorities recently provided updated estimates on claims of about US\$1.3 billion at end-2008 (11 percent of GDP). These claims are mainly from suppliers, public enterprises and public sector employees dating back to the period of conflict in the 1990s. The authorities are in the process of developing a strategy — to be finalized in mid 2010 - to address with these claims.

\section{Conclusion}

45. The DRC would remain in debt distress in the absence of substantial debt relief. The external debt indicators would breach policy-dependent thresholds over prolonged periods even with a strong economic recovery over the medium term (assumed under the baseline scenario). Even if the DRC were to reach the HIPC completion point in mid-2010 and benefit from HIPC/MDRI debt relief, the external debt outlook remain vulnerable to adverse shocks. The public guarantee on the SCCA financing increases the external debt-toGDP ratio significantly above the policy-dependent threshold level for a prolonged period. However, these calculations substantially overstate the risk of debt distress because they assume that the mining project will not generate any net operating profits over the entire 25-year projection period. Calculations based on a model of income generated by the mining project developed by the DRC authorities predict that the net operating profits from the mining project would fully repay the public infrastructure loans by 2018, 16 years before the public guarantee would be invoked. Net operating income would have to decline by 65 percent of the amount projected in the baseline scenario in order for the public guarantee to be invoked. These calculations indicate that the risk for debt sustainability is manageable.

conventional loan with a fixed repayment schedule, compared to only \$267 million in the case where the public guarantee can only be invoked after 25 years. 
Figure 1. Democratic Republic of the Congo: Indicators of Public and Publicly Guaranteed External Debt without Debt Relief, 2009-29
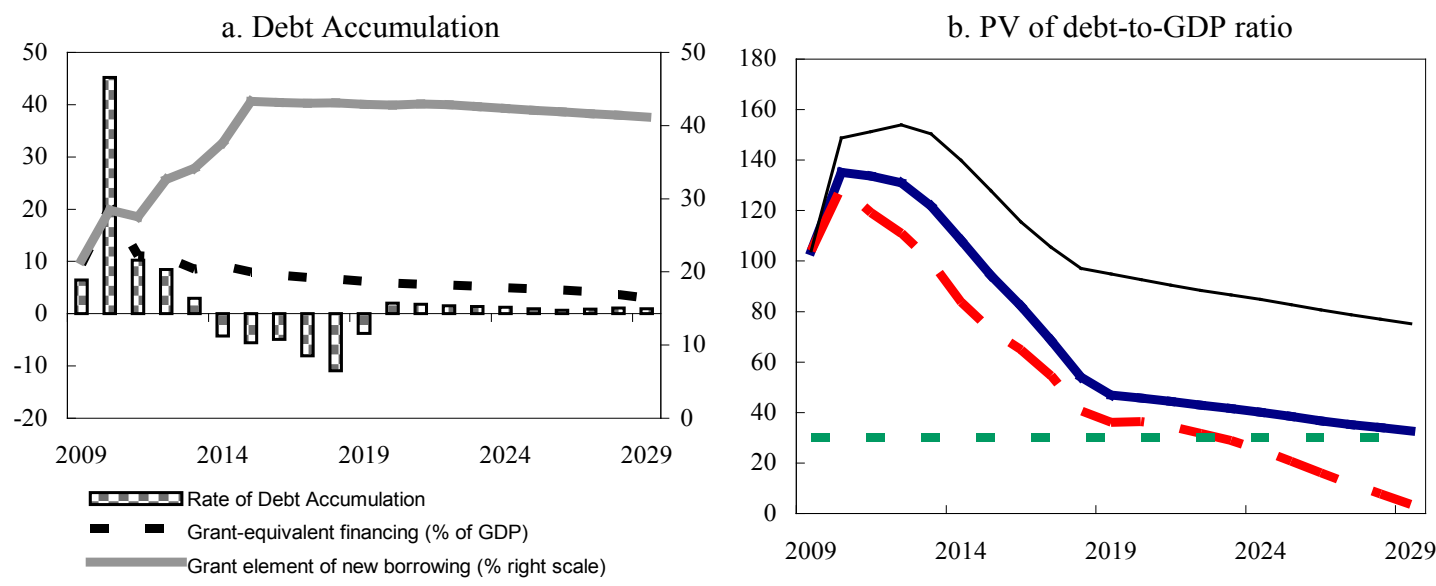

c. PV of debt-to-exports ratio

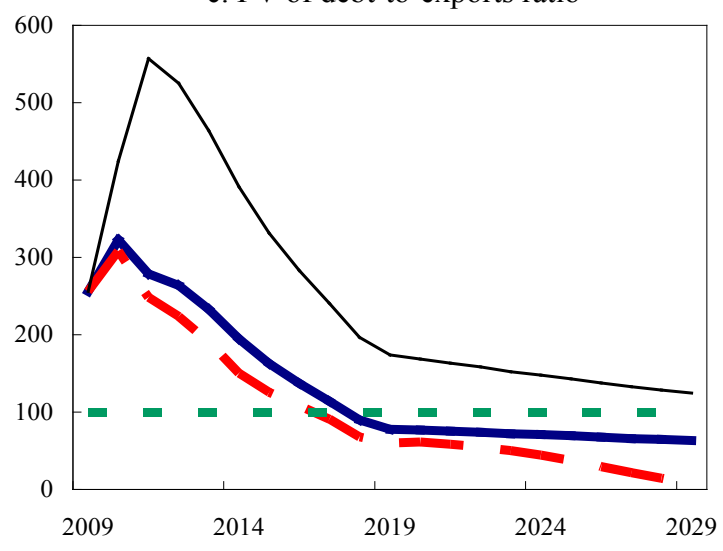

d. PV of debt-to-revenue ratio
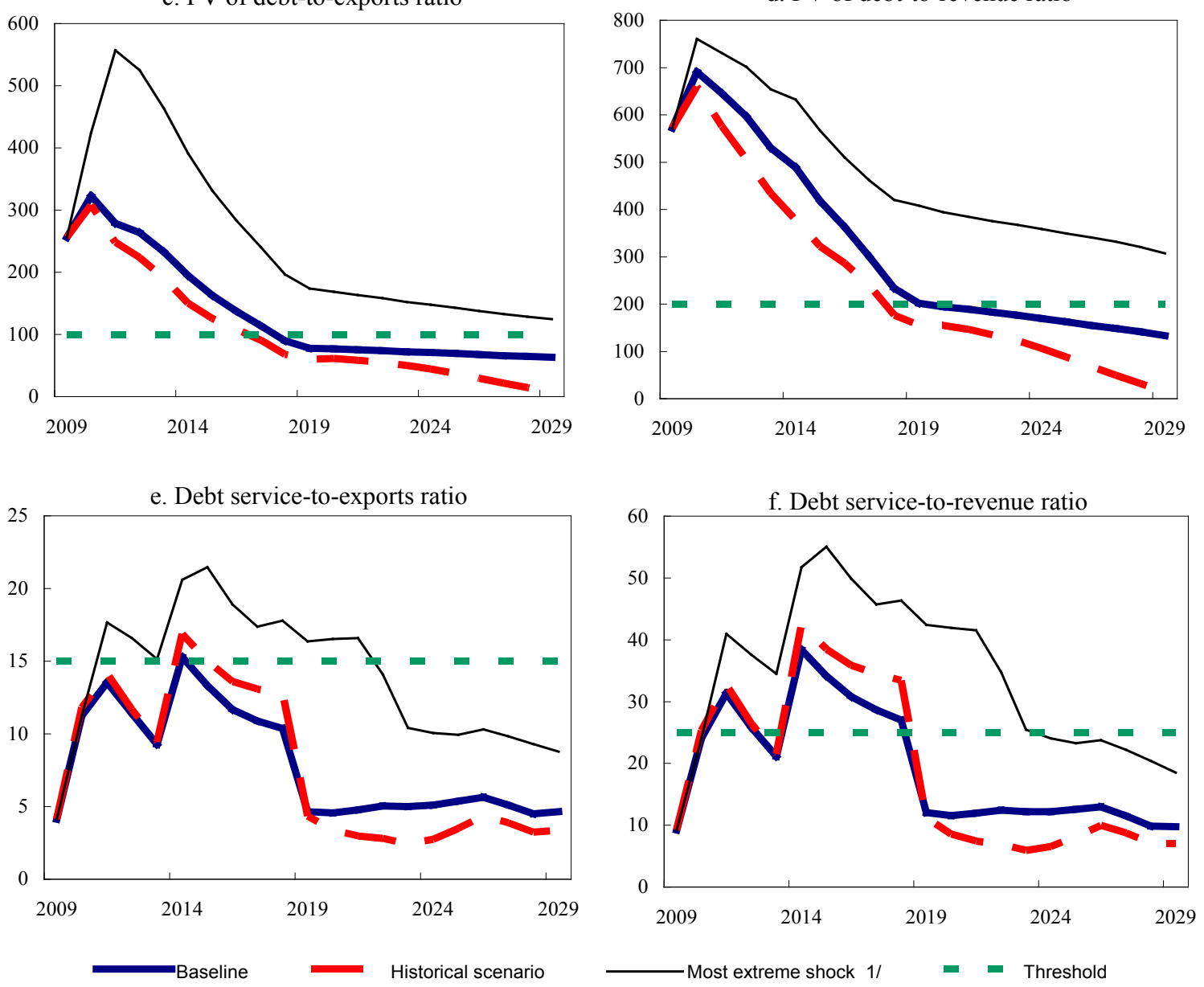

Source: IMF staff projections and simulations.

$1 /$ The most extreme stress test is the test that yields the highest ratio in 2019, which is new public sector loans on less favorable terms in figures $b, d$ and $f$ and lower exports in figures $c$ and $e$. 
Figure 2. Democratic Republic of the Congo: Indicators of Public and Publicly Guaranteed External Debt - HIPC Completion Point Scenario, 2009-29
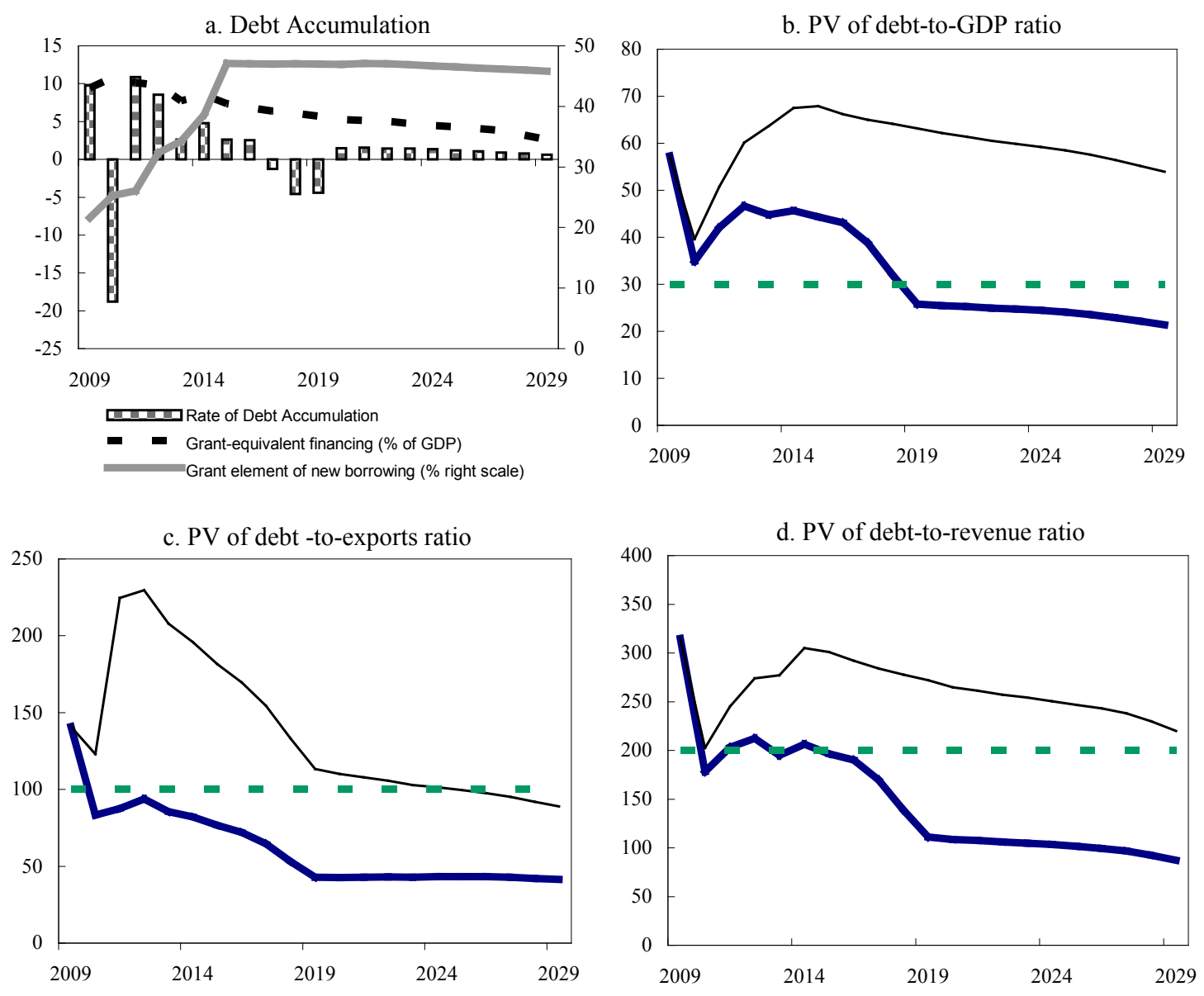

e. Debt service-to-exports ratio
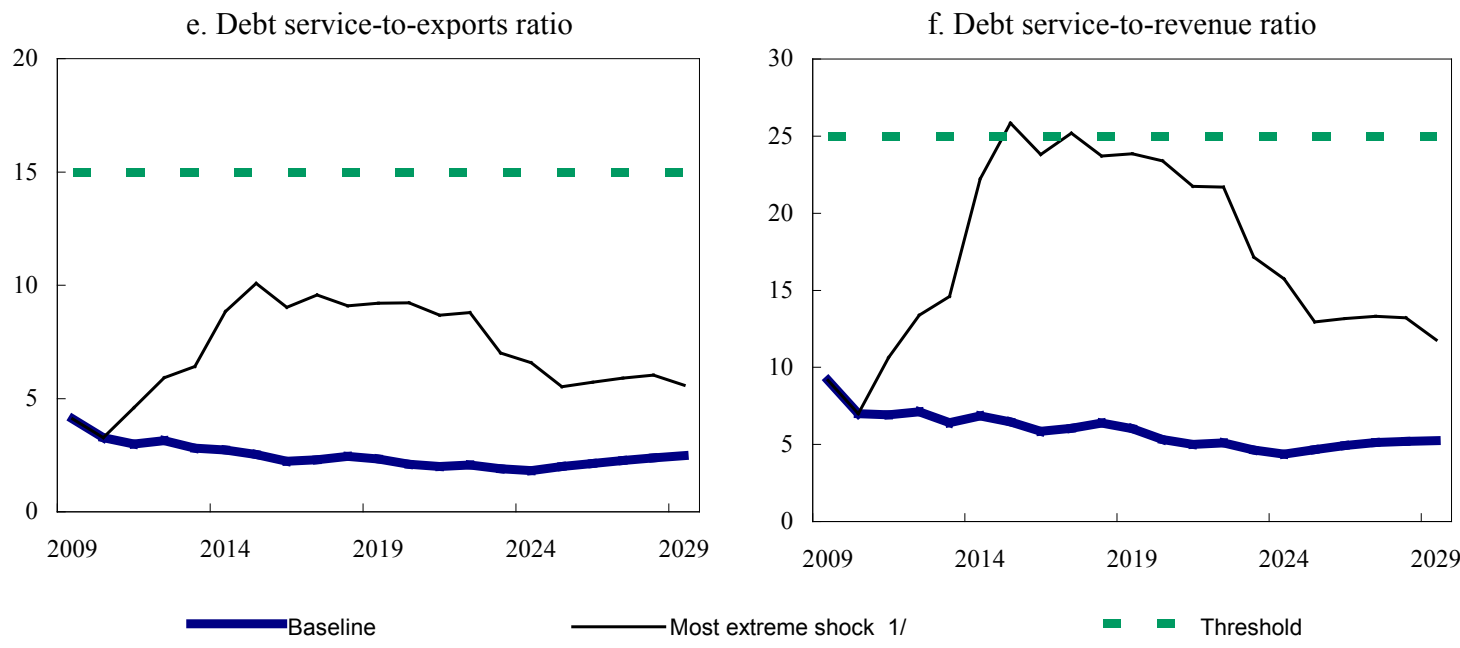

Source: IMF staff projections and simulations.

$1 /$ The most extreme stress test is the test that yields the highest ratio in 2019 , which is new public sector loans on less favorable terms in figures $b$ to $f$. 
Figure 3. Democratic Republic of the Congo: Indicators of Public and Publicly Guaranteed External Debt HIPC Completion Point Scenario with Alternative Valuations of Public Guarantee, 2009-29

a. PV of debt-to-GDP ratio

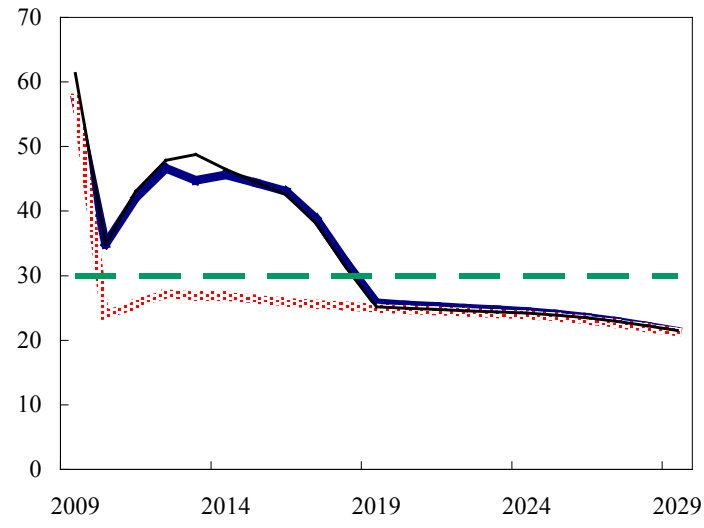

b. PV of debt-to-exports ratio

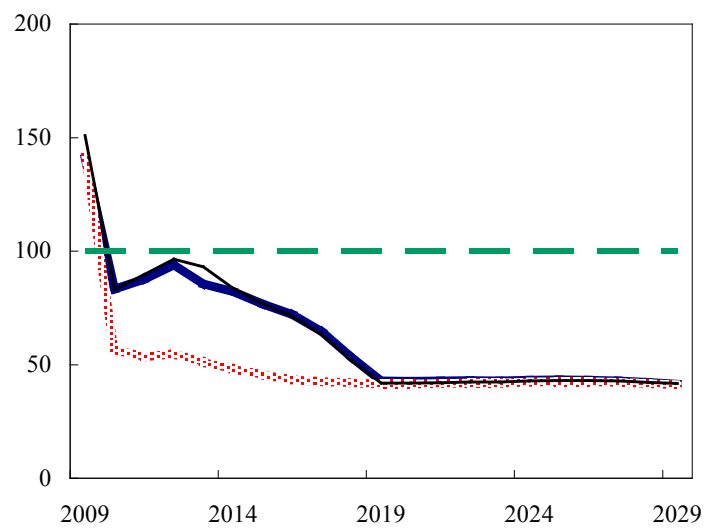

d. Debt service-to-exports ratio

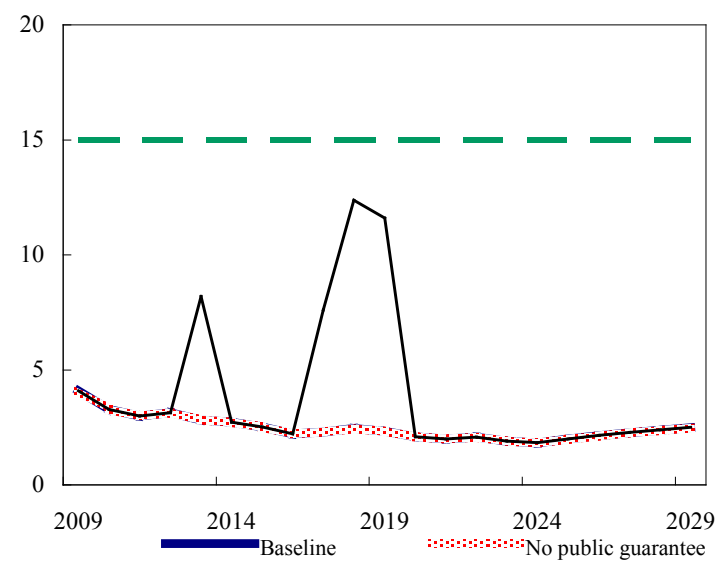

c. PV of debt-to-revenue ratio
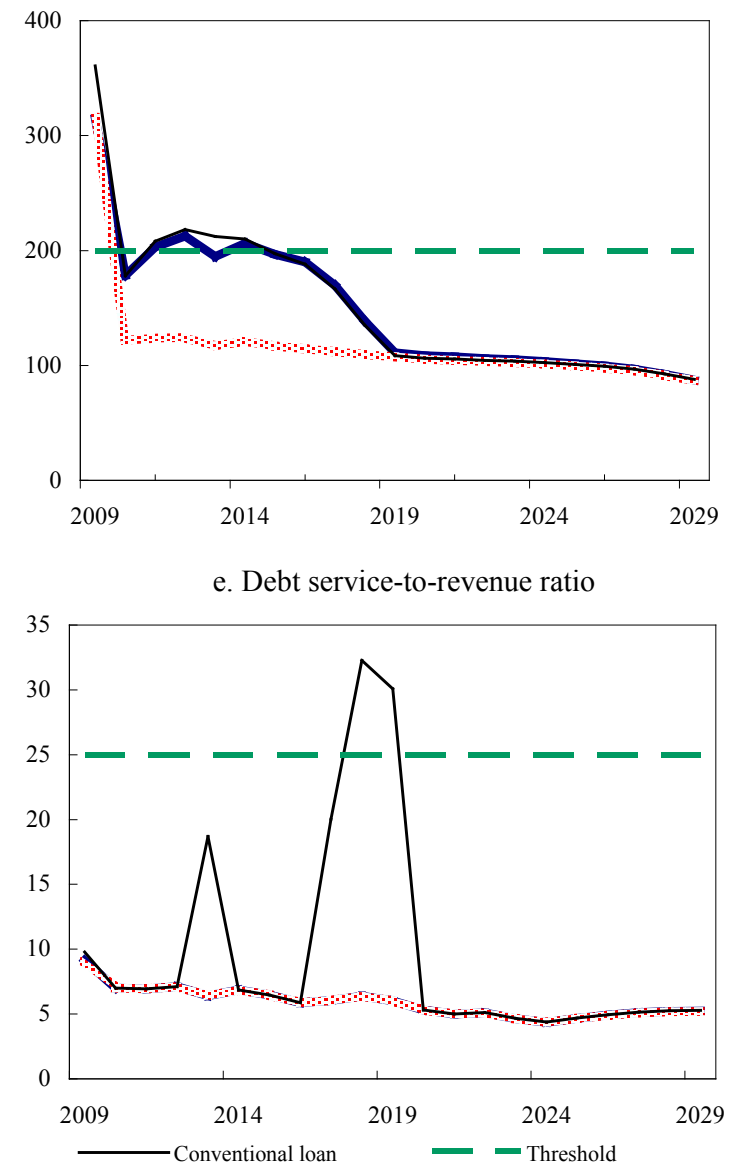

Sources: Congolese authorities; and IMF staff estimates and projections. 
Figure 4. Democratic Republic of the Congo: Indicators of Public Debt without Debt Relief, 2009-29

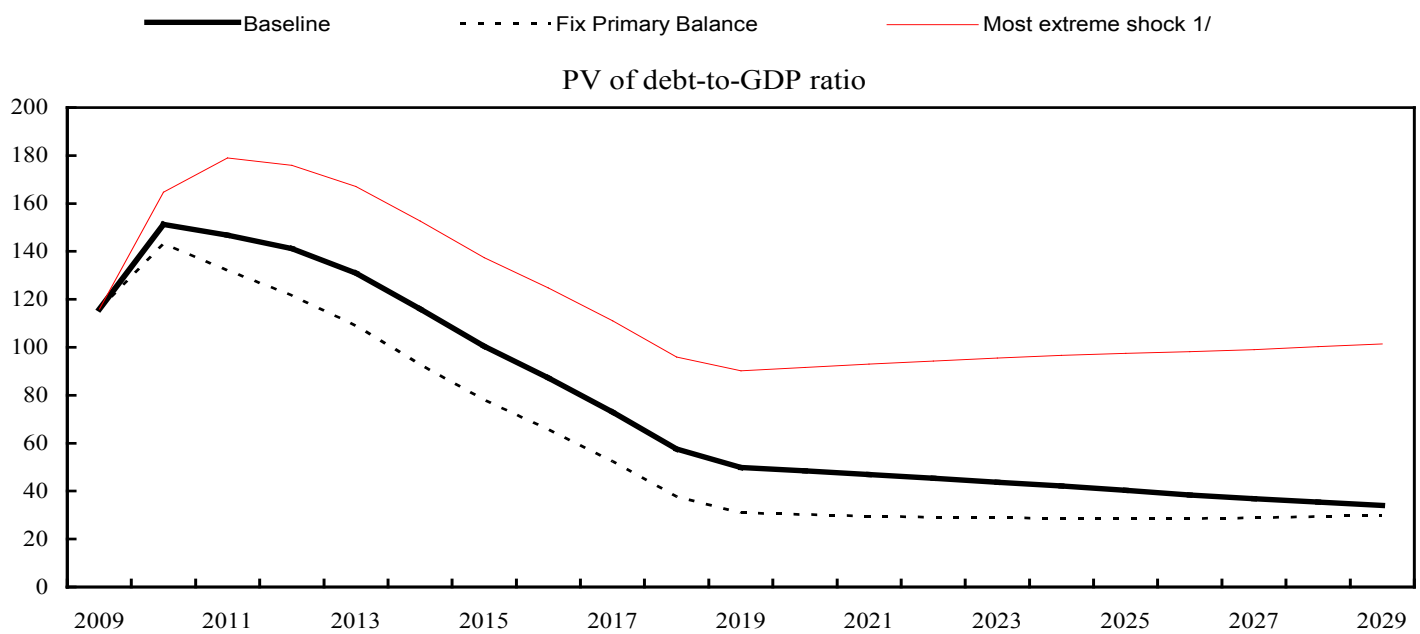

PV of debt-to-revenue ratio

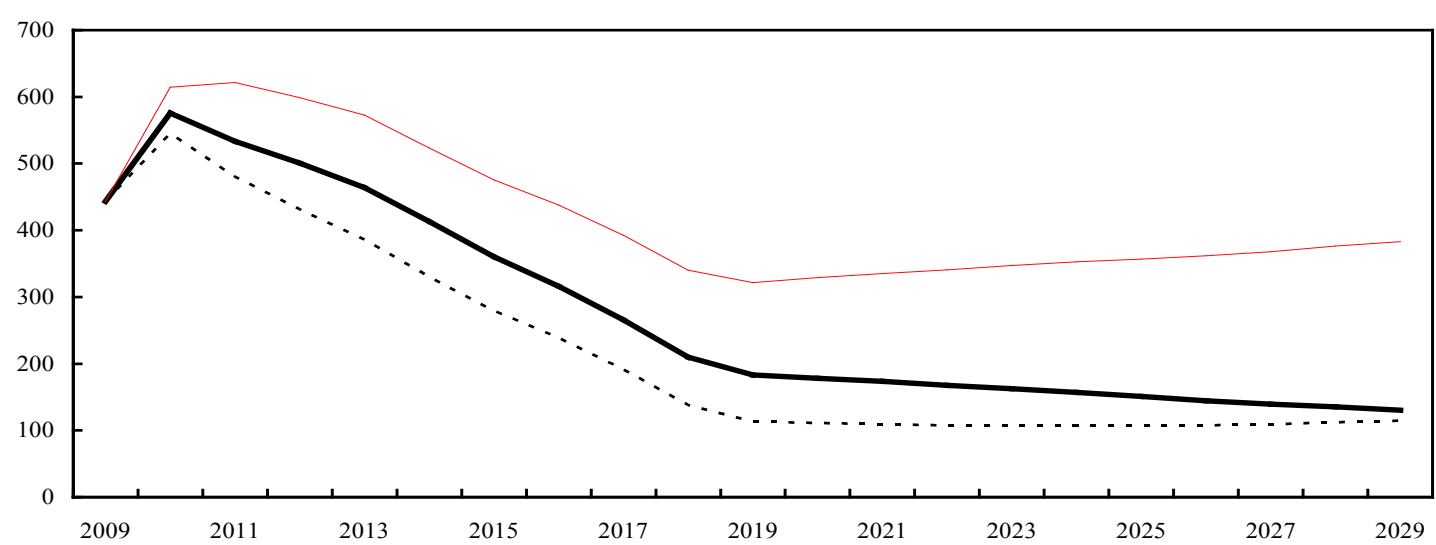

Debt service-to-revenue ratio $2 /$

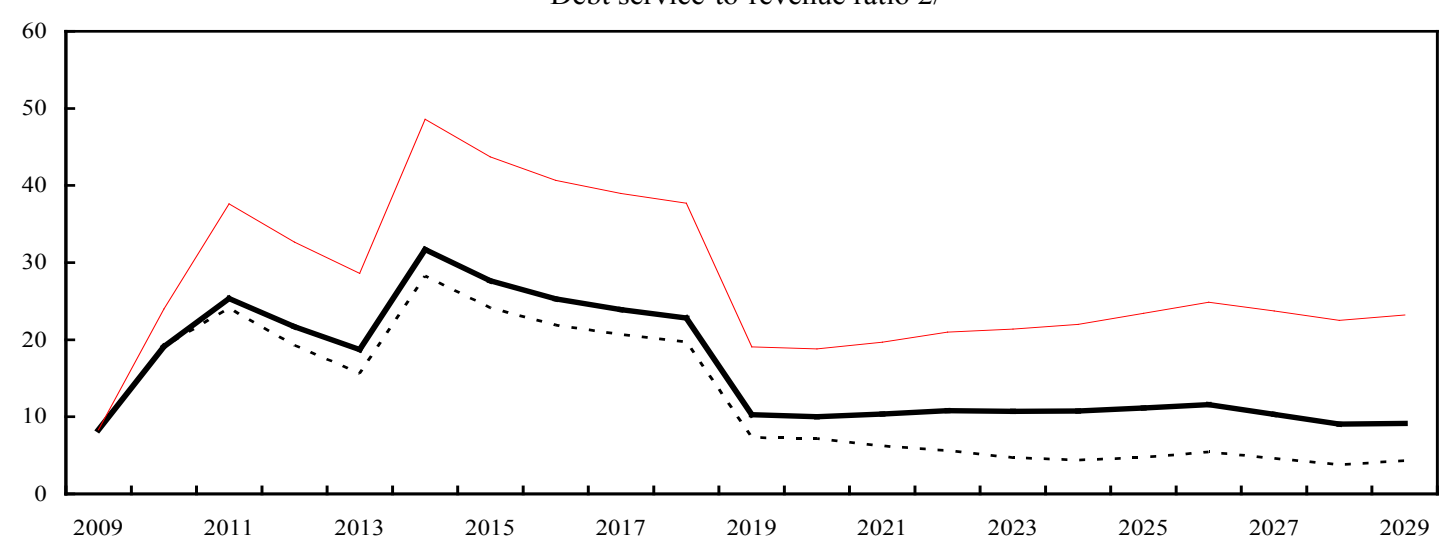

Sources: Congolese authorities; and IMF staff estimates and projections.

1/ The most extreme stress test is the test that yields the highest ratio in 2019.

2/ Revenues are defined inclusive of grants. 
Table 1. Democratic Republic of the Congo: External Debt Sustainability Framework, Baseline Scenario, 2008-29 1/ (In percent of GDP, unless otherwise indicated)

\begin{tabular}{|c|c|c|c|c|c|c|c|c|c|c|c|c|c|}
\hline & \multirow{2}{*}{$\begin{array}{r}\text { Actual } \\
2008 \\
\end{array}$} & \multirow{2}{*}{$\begin{array}{l}\text { Historical } \\
\text { Average }\end{array}$} & \multirow{2}{*}{$\begin{array}{l}\text { Standard } \\
\text { Deviation }\end{array}$} & \multicolumn{6}{|c|}{ Projections } & \multirow[b]{2}{*}{$\begin{array}{l}2009-14 \\
\text { Average }\end{array}$} & \multirow[b]{2}{*}{2019} & \multirow[b]{2}{*}{2029} & \multirow[b]{2}{*}{$\begin{array}{l}2015-29 \\
\text { Average }\end{array}$} \\
\hline & & & & 2009 & 2010 & 2011 & 2012 & 2013 & 2014 & & & & \\
\hline External debt (nominal) 1/ & 128.4 & & & 138.7 & 154.2 & 165.8 & 170.2 & 164.7 & 157.5 & & 77.7 & 54.0 & \\
\hline $\mathrm{o} / \mathrm{w}$ public and publicly guaranteed (PPG) & 128.4 & & & 132.4 & 138.8 & 139.1 & 138.1 & 131.0 & 123.0 & & 74.9 & 54.0 & \\
\hline Change in external debt & 3.8 & & & 10.3 & 15.4 & 11.6 & 4.5 & -5.6 & -7.2 & & -12.8 & -2.1 & \\
\hline Identified net debt-creating flows & -19.1 & & & 4.8 & 9.7 & 7.8 & 2.8 & -2.6 & -3.7 & & -6.0 & 2.3 & \\
\hline Non-interest current account deficit & 12.7 & 2.6 & 4.9 & 13.7 & 21.2 & 23.2 & 17.6 & 13.7 & 10.4 & & 2.7 & 6.9 & 5.6 \\
\hline Deficit in balance of goods and services & 14.9 & & & 23.8 & 27.9 & 31.5 & 24.6 & 18.6 & 14.3 & & 2.6 & 6.1 & \\
\hline Exports & 61.6 & & & 40.6 & 41.8 & 48.0 & 49.7 & 52.4 & 55.6 & & 60.2 & 51.7 & \\
\hline Imports & 76.4 & & & 64.5 & 69.8 & 79.4 & 74.2 & 70.9 & 69.8 & & 62.7 & 57.8 & \\
\hline Net current transfers (negative $=$ inflow) & -10.6 & -6.7 & 2.6 & -13.9 & -10.0 & -9.5 & -8.7 & -7.1 & -6.5 & & -5.1 & -3.7 & -4.7 \\
\hline o/w official & -8.8 & & & -12.8 & -9.4 & -8.8 & -8.0 & -6.4 & -5.7 & & -3.9 & -2.2 & \\
\hline Other current account flows $($ negative $=$ net inflow) & 8.5 & & & 3.8 & 3.3 & 1.2 & 1.7 & 2.2 & 2.7 & & 5.2 & 4.4 & \\
\hline Net FDI (negative = inflow) & -14.8 & -4.8 & 4.3 & -5.7 & -6.5 & -8.4 & -7.1 & -6.1 & -6.1 & & -5.7 & -2.7 & -4.6 \\
\hline Endogenous debt dynamics 21 & -17.0 & -4.0 & 4.0 & $\begin{array}{l}-3.1 \\
-3.3\end{array}$ & -5.0 & -7.0 & -7.6 & -10.2 & -8.0 & & $\begin{array}{l}-3.1 \\
-3.0\end{array}$ & -1.8 & -4.0 \\
\hline Contribution from nominal interest rate & 0.3 & & & 0.3 & 1.8 & 3.3 & 2.9 & 2.6 & 2.4 & & 1.1 & 0.5 & \\
\hline Contribution from real GDP growth & -6.6 & & & -3.6 & -6.7 & -10.2 & -10.5 & -12.8 & -10.4 & & -4.1 & -2.3 & \\
\hline Contribution from price and exchange rate changes & -10.7 & & & & & & & & & & & & \\
\hline $\begin{array}{l}\text { Residual (3-4) } 3 / \\
\text { o/w excoptional financing }\end{array}$ & $\begin{array}{l}22.9 \\
-60\end{array}$ & & & $\begin{array}{r}5.6 \\
-7.3\end{array}$ & $\begin{array}{r}5.7 \\
-356\end{array}$ & $\begin{array}{r}3.8 \\
-4.3\end{array}$ & $\begin{array}{r}1.6 \\
-3.4\end{array}$ & $\begin{array}{l}-2.9 \\
-2.7\end{array}$ & $\begin{array}{r}-3.4 \\
2.0\end{array}$ & & $\begin{array}{l}-6.8 \\
-12\end{array}$ & $\begin{array}{l}-4.4 \\
-0.4\end{array}$ & \\
\hline $\begin{array}{l}\text { O/W exceptional financing } \\
\text { PV of external debt } 4 /\end{array}$ & $\begin{array}{l}-6.0 \\
92.6\end{array}$ & & & $\begin{array}{r}-1.3 \\
110.4\end{array}$ & $\begin{array}{r}-35.6 \\
150.5\end{array}$ & $\begin{array}{r}-4.3 \\
160.4\end{array}$ & $\begin{array}{r}-3.4 \\
163.1\end{array}$ & 155.6 & 142.8 & & 49.7 & $\begin{array}{r}-0.4 \\
32.7\end{array}$ & \\
\hline In percent of exports & $\begin{array}{r}92.6 \\
150.4\end{array}$ & & & 271.7 & 359.6 & 334.3 & 328.5 & 297.1 & 256.9 & & 82.6 & 63.3 & \\
\hline PV of PPG external debt & 92.6 & & & 104.1 & 135.1 & 133.7 & 131.0 & 121.8 & 108.2 & & 46.9 & 32.7 & \\
\hline In percent of exports & 150.4 & & & 256.1 & 322.9 & 278.6 & 263.8 & 232.7 & 194.7 & & 78.0 & 63.4 & \\
\hline In percent of government revenues & 501.5 & & & 572.3 & 690.9 & 645.6 & 596.9 & 530.1 & 489.1 & & 202.1 & 133.5 & \\
\hline Debt service-to-exports ratio (in percent) & 3.6 & & & 4.1 & 11.3 & 13.5 & 11.4 & 12.8 & 19.4 & & 12.4 & 4.6 & \\
\hline PPG debt service-to-exports ratio (in percent) & 3.6 & & & 4.1 & 11.3 & 13.5 & 11.4 & 9.3 & 15.3 & & 4.6 & 4.6 & \\
\hline PPG debt service-to-revenue ratio (in percent) & 11.9 & & & 9.2 & 24.1 & 31.4 & 25.8 & 21.1 & 38.4 & & 12.0 & 9.8 & \\
\hline Total gross financing need (Billions of U.S. dollars) & 0.0 & & & 1.1 & 2.4 & 2.8 & 2.3 & 2.3 & 2.6 & & 1.1 & 3.2 & \\
\hline Non-interest current account deficit that stabilizes debt ratio & 8.9 & & & 3.4 & 5.8 & 11.6 & 13.1 & 19.2 & 17.6 & & 15.5 & 8.9 & \\
\hline \multicolumn{14}{|l|}{ Key macroeconomic assumptions } \\
\hline Real GDP growth (in percent) & 6.2 & 2.8 & 5.3 & 2.7 & 5.4 & 7.2 & 6.9 & 8.2 & 6.8 & 6.2 & 4.8 & 4.4 & 4.9 \\
\hline GDP deflator in US dollar terms (change in percent) & 9.4 & 2.3 & 13.8 & -7.3 & 4.8 & 1.5 & 1.5 & 1.6 & 1.6 & 0.6 & 2.2 & 2.2 & 2.1 \\
\hline Effective interest rate (percent) 5/ & 0.3 & 0.3 & 0.1 & 0.2 & 1.4 & 2.3 & 1.9 & 1.7 & 1.6 & 1.5 & 1.3 & 0.9 & 1.1 \\
\hline Growth of exports of G\&S (US dollar terms, in percent) & 8.7 & 22.0 & 31.7 & -37.2 & 13.8 & 24.7 & 12.3 & 15.9 & 15.3 & 7.5 & 7.0 & 4.7 & 6.7 \\
\hline Growth of imports of G\&S (US dollar terms, in percent) & 28.8 & 25.2 & 31.8 & -19.7 & 19.6 & 23.9 & 1.4 & 5.1 & 6.9 & 6.2 & 6.6 & 4.8 & 5.8 \\
\hline Grant element of new public sector borrowing (in percent) & & $\ldots$ & $\ldots$ & 21.6 & 28.4 & 27.5 & 32.6 & 34.2 & 37.6 & 30.3 & 42.9 & 41.2 & 42.5 \\
\hline Government revenues (excluding grants, in percent of GDP) & 18.5 & & & 18.2 & 19.6 & 20.7 & 21.9 & 23.0 & 22.1 & & 23.2 & 24.5 & 23.5 \\
\hline Aid flows (in Billions of US dollars) 6/ & 0.4 & & & 1.6 & 1.5 & 1.8 & 1.5 & 1.6 & 1.7 & & 2.0 & & \\
\hline o/w Grants & 0.2 & & & 0.9 & 0.8 & 0.9 & 0.9 & 0.8 & 1.0 & & 1.0 & 0.8 & \\
\hline o/w Concessional loans & 0.1 & & & 0.8 & 0.7 & 0.9 & 0.6 & 0.7 & 0.7 & & 1.0 & & \\
\hline Grant-equivalent financing (in percent of GDP) 7/ & & & & 9.4 & 20.5 & 11.1 & 10.6 & 8.5 & 9.1 & & 6.3 & 3.1 & 5.5 \\
\hline Grant-equivalent financing (in percent of external financing) $7 /$ & $\cdots$ & & & 64.1 & 37.1 & 49.6 & 54.0 & 57.4 & 63.3 & & 68.1 & 60.6 & 67.0 \\
\hline \multicolumn{14}{|l|}{ Memoran } \\
\hline Nominal GDP (Billions of US dollars) & 11.6 & & & 11.0 & 12.2 & 13.3 & 14.4 & 15.8 & 17.2 & & 25.1 & 48.3 & \\
\hline Nominal dollar GDP growth & 16.1 & & & -4.8 & 10.4 & 8.8 & 8.5 & 9.9 & 8.6 & 6.9 & 7.1 & 6.7 & 7.1 \\
\hline PV of PPG external debt (in Billions of US dollars) & 10.7 & & & 11.5 & 16.5 & 17.7 & 18.8 & 19.3 & 18.6 & & 11.8 & 15.8 & \\
\hline (PVt-PVt-1)/GDPt-1 (in percent) & & & & 6.4 & 45.2 & 10.3 & 8.5 & 2.9 & -4.3 & 11.5 & -3.8 & 0.9 & -1.4 \\
\hline
\end{tabular}

Source: IMF staff simulations.

1/ Includes both public and private sector external debt.

$3 /$ Includes exceptional financing (i.e., changes in arrears and debt relief); changes in gross foreign assets; and valuation adjustments. For projections also includes contribution from price and exchange rate changes. 4/ Assumes that PV of private sector debt is equivalent to its face value.

6/ Current-year interest payments divided by previous peris as grants, concessional loans, and debt relief.

7/ Grant-equivalent financing includes grants provided directly to the government and through new borrowing (difference between the face value and the PV of new debt). 
Table 2. Democratic Republic of the Congo: Sensitivity Analysis for Key Indicators of Public and Publicly Guaranteed External Debt, 2009-29 (In percent)

\begin{tabular}{|c|c|c|c|c|c|c|c|c|c|c|c|c|}
\hline & \multicolumn{12}{|c|}{ Projections } \\
\hline & 2009 & 2010 & 2011 & 2012 & 2013 & 2014 & 2015 & 2016 & 2017 & 2018 & 2019 & 2029 \\
\hline \multicolumn{13}{|c|}{ PV of debt-to GDP ratio } \\
\hline Baseline & 104.1 & 135.1 & 133.7 & 131.0 & 121.8 & 108.2 & 94.4 & 82.1 & 68.7 & 54.0 & 46.9 & 32.7 \\
\hline \multicolumn{13}{|l|}{ A. Alternative Scenarios } \\
\hline A1. Key variables at their historical averages in 2009-2029 1/ & 104.1 & 129.0 & 119.2 & 111.0 & 99.8 & 83.6 & 72.6 & 64.9 & 54.7 & 40.8 & 36.1 & 3.5 \\
\hline A2. New public sector loans on less favorable terms in 2009-2029 2/ & 104.1 & 148.7 & 151.3 & 154.0 & 150.4 & 139.9 & 127.9 & 115.5 & 105.6 & 97.1 & 94.8 & 75.3 \\
\hline \multicolumn{13}{|l|}{ B. Bound Tests } \\
\hline B1. Real GDP growth at historical average minus one standard deviation in 2010-2011 & 104.1 & 145.9 & 158.4 & 155.1 & 144.2 & 128.1 & 111.6 & 97.0 & 81.2 & 63.7 & 55.3 & 38.6 \\
\hline B2. Export value growth at historical average minus one standard deviation in 2010-2011 3/ & 104.1 & 141.2 & 153.6 & 149.9 & 139.6 & 125.1 & 110.3 & 97.2 & 83.3 & 68.0 & 60.2 & 37.0 \\
\hline B3. US dollar GDP deflator at historical average minus one standard deviation in 2010-2011 & 104.1 & 160.0 & 181.4 & 177.7 & 165.2 & 146.7 & 127.8 & 111.1 & 93.0 & 73.0 & 63.3 & 44.2 \\
\hline $\begin{array}{l}\text { B4. Net non-debt creating flows at historical average minus one standard deviation in 2010-2011 } 4 /\end{array}$ & 104.1 & 143.5 & 150.9 & 147.3 & 137.1 & 122.8 & 108.1 & 95.1 & 81.2 & 66.1 & 58.2 & 36.3 \\
\hline B5. Combination of B1-B4 using one-half standard deviation shocks & 104.1 & 166.1 & 206.5 & 201.4 & 187.6 & 168.3 & 148.7 & 131.3 & 112.8 & 92.7 & 82.2 & 49.6 \\
\hline B6. One-time 30 percent nominal depreciation relative to the baseline in $20105 /$ & 104.1 & 189.3 & 187.0 & 183.2 & 170.3 & 151.2 & 131.8 & 114.6 & 95.8 & 75.2 & 65.3 & 45.6 \\
\hline \multicolumn{13}{|c|}{ PV of debt-to-exports ratio } \\
\hline Baseline & 256.1 & 322.9 & 278.6 & 263.8 & 232.7 & 194.7 & 163.0 & 137.4 & 114.2 & 89.7 & 78.0 & 63.4 \\
\hline \multicolumn{13}{|l|}{ A. Alternative Scenarios } \\
\hline A1. Key variables at their historical averages in 2009-2029 1/ & 256.1 & 308.3 & 248.4 & 223.5 & 190.5 & 150.4 & 125.4 & 108.5 & 90.8 & 67.7 & 60.0 & 6.8 \\
\hline A2. New public sector loans on less favorable terms in 2009-2029 $2 /$ & 256.1 & 355.4 & 315.5 & 310.1 & 287.2 & 251.7 & 221.0 & 193.2 & 175.3 & 161.3 & 157.7 & 145.7 \\
\hline \multicolumn{13}{|l|}{ B. Bound Tests } \\
\hline B1. Real GDP growth at historical average minus one standard deviation in 2010-2011 & 256.1 & 322.8 & 278.3 & 263.4 & 232.2 & 194.2 & 162.5 & 136.8 & 113.6 & 89.2 & 77.5 & 63.0 \\
\hline B2. Export value growth at historical average minus one standard deviation in 2010-2011 3/ & 256.1 & 424.9 & 556.8 & 525.0 & 463.6 & 391.3 & 331.5 & 282.9 & 240.5 & 196.5 & 174.1 & 124.6 \\
\hline B3. US dollar GDP deflator at historical average minus one standard deviation in 2010-2011 & 256.1 & 322.8 & 278.3 & 263.4 & 232.2 & 194.2 & 162.5 & 136.8 & 113.6 & 89.2 & 77.5 & 63.0 \\
\hline B4. Net non-debt creating flows at historical average minus one standard deviation in 2010-2011 4/ & 256.1 & 342.9 & 314.5 & 296.7 & 261.9 & 220.9 & 186.8 & 159.2 & 134.9 & 109.8 & 96.8 & 70.4 \\
\hline B5. Combination of B1-B4 using one-half standard deviation shocks & 256.1 & 368.2 & 412.1 & 388.3 & 343.0 & 289.9 & 246.0 & 210.4 & 179.4 & 147.4 & 130.7 & 92.0 \\
\hline B6. One-time 30 percent nominal depreciation relative to the baseline in 20105 / & 256.1 & 322.8 & 278.3 & 263.4 & 232.2 & 194.2 & 162.5 & 136.8 & 113.6 & 89.2 & 77.5 & 63.0 \\
\hline \multicolumn{13}{|c|}{ PV of debt-to-revenue ratio } \\
\hline Baseline & 572.3 & 690.9 & 645.6 & 596.9 & 530.1 & 489.1 & 418.0 & 362.2 & 300.4 & 233.9 & 202.1 & 133.5 \\
\hline \multicolumn{13}{|l|}{ A. Alternative Scenarios } \\
\hline A1. Key variables at their historical averages in 2009-2029 1/ & 572.3 & 659.6 & 575.7 & 505.8 & 434.0 & 377.6 & 321.6 & 286.2 & 238.8 & 176.4 & 155.4 & 14.3 \\
\hline A2. New public sector loans on less favorable terms in 2009-2029 $2 /$ & 572.3 & 760.4 & 731.0 & 701.6 & 654.2 & 632.1 & 566.6 & 509.6 & 461.3 & 420.2 & 408.4 & 307.0 \\
\hline \multicolumn{13}{|l|}{ B. Bound Tests } \\
\hline B1. Real GDP growth at historical average minus one standard deviation in 2010-2011 & 572.3 & 745.8 & 765.1 & 706.9 & 627.5 & 578.7 & 494.2 & 428.1 & 354.7 & 275.7 & 238.0 & 157.4 \\
\hline B2. Export value growth at historical average minus one standard deviation in 2010-2011 3/ & 572.3 & 721.7 & 742.0 & 683.1 & 607.3 & 565.2 & 488.7 & 429.0 & 363.8 & 294.5 & 259.3 & 151.0 \\
\hline B3. US dollar GDP deflator at historical average minus one standard deviation in 2010-2011 & 572.3 & 818.3 & 876.2 & 809.6 & 718.7 & 662.8 & 566.0 & 490.3 & 406.2 & 315.8 & 272.6 & 180.2 \\
\hline B4. Net non-debt creating flows at historical average minus one standard deviation in 2010-2011 4/ & 572.3 & 733.6 & 728.8 & 671.3 & 596.6 & 554.7 & 478.9 & 419.8 & 355.0 & 286.1 & 250.8 & 148.2 \\
\hline B5. Combination of B1-B4 using one-half standard deviation shocks & 572.3 & 849.4 & 997.4 & 917.8 & 816.1 & 760.6 & 658.8 & 579.4 & 492.9 & 401.1 & 353.7 & 202.5 \\
\hline B6. One-time 30 percent nominal depreciation relative to the baseline in 20105 / & 572.3 & 967.7 & 903.5 & 834.9 & 741.1 & 683.4 & 583.7 & 505.6 & 418.8 & 325.6 & 281.1 & 185.8 \\
\hline \multicolumn{13}{|c|}{ Debt service-to-exports ratio } \\
\hline Baseline & 4.1 & 11.3 & 13.5 & 11.4 & 9.3 & 15.3 & 13.3 & 11.7 & 10.9 & 10.4 & 4.6 & 4.6 \\
\hline \multicolumn{13}{|l|}{ A. Alternative Scenarios } \\
\hline A1. Key variables at their historical averages in 2009-2029 1/ & 4.1 & 11.8 & 14.1 & 11.7 & 9.4 & 16.9 & 15.0 & 13.6 & 13.1 & 12.8 & 4.3 & 3.4 \\
\hline A2. New public sector loans on less favorable terms in 2009-2029 $2 /$ & 4.1 & 11.3 & 17.7 & 16.6 & 15.1 & 20.6 & 21.5 & 18.9 & 17.4 & 17.8 & 16.4 & 8.8 \\
\hline \multicolumn{13}{|l|}{ B. Bound Tests } \\
\hline B1. Real GDP growth at historical average minus one standard deviation in 2010-2011 & 4.1 & 11.3 & 13.5 & 11.4 & 9.3 & 15.3 & 13.3 & 11.7 & 10.9 & 10.4 & 4.6 & 4.6 \\
\hline B2. Export value growth at historical average minus one standard deviation in 2010-2011 3/ & 4.1 & 14.2 & 24.0 & 21.2 & 17.4 & 27.7 & 24.1 & 21.2 & 19.8 & 18.8 & 9.5 & 10.0 \\
\hline B3. US dollar GDP deflator at historical average minus one standard deviation in 2010-2011 & 4.1 & 11.3 & 13.5 & 11.4 & 9.3 & 15.3 & 13.3 & 11.7 & 10.9 & 10.4 & 4.6 & 4.6 \\
\hline B4. Net non-debt creating flows at historical average minus one standard deviation in 2010-2011 4/ & 4.1 & 11.3 & 13.9 & 12.1 & 9.9 & 15.8 & 13.8 & 12.1 & 11.3 & 10.8 & 5.5 & 5.6 \\
\hline B5. Combination of B1-B4 using one-half standard deviation shocks & 4.1 & 12.1 & 17.5 & 15.6 & 12.7 & 20.2 & 17.6 & 15.4 & 14.4 & 13.7 & 7.2 & 7.5 \\
\hline B6. One-time 30 percent nominal depreciation relative to the baseline & 4.1 & 11.3 & 13.5 & 11.4 & 9.3 & 15.3 & 13.3 & 11.7 & 10.9 & 10.4 & 4.6 & 4.6 \\
\hline
\end{tabular}

Debt service-to-revenue ratio

Baseline

$\begin{array}{rrrrrrrrrrrr}9.2 & 24.1 & 31.4 & 25.8 & 21.1 & 38.4 & 34.2 & 30.8 & 28.7 & 27.0 & \mathbf{1 2 . 0} & 9.8 \\ & & & & & & & & & & & \\ 9.2 & 25.3 & 32.7 & 26.4 & 21.5 & 42.4 & 38.5 & 35.8 & 34.4 & 33.4 & \mathbf{1 1 . 2} & 7.1 \\ 9.2 & 24.1 & 40.9 & 37.5 & 34.5 & 51.7 & 55.0 & 49.9 & 45.7 & 46.3 & \mathbf{4 2 . 4} & 18.5 \\ & & & & & & & & & & & \\ & & & & & & & & & & & \\ 9.2 & 26.0 & 37.2 & 30.6 & 25.0 & 45.5 & 40.5 & 36.5 & 34.0 & 32.0 & 14.2 & 11.5 \\ 9.2 & 24.1 & 31.9 & 27.6 & 22.8 & 40.0 & 35.6 & 32.1 & 29.9 & 28.2 & 14.1 & 12.1 \\ 9.2 & 28.6 & 42.6 & 35.0 & 28.7 & 52.1 & 46.4 & 41.8 & 38.9 & 36.7 & 16.3 & 13.2 \\ 9.2 & 24.1 & 32.2 & 27.4 & 22.5 & 39.7 & 35.4 & 31.9 & 29.7 & 28.0 & 14.4 & 11.8 \\ 9.2 & 27.8 & 42.4 & 36.8 & 30.3 & 5.9 & 47.1 & 42.5 & 39.6 & 37.3 & 19.4 & 16.4 \\ 9.2 & 33.8 & 43.9 & 36.1 & 29.6 & 53.8 & 47.8 & 43.1 & 40.1 & 37.8 & 16.8 & 13.6 \\ & & & & & & & & & & & \end{array}$

A1. Key variables at their historical averages in 2009-2029 $1 /$

A2. New public sector loans on less favorable terms in 2009-2029 2

B. Bound Tests

B1. Real GDP growth at historical average minus one standard deviation in 2010-2011

B2. Export value growth at historical average minus one standard deviation in 2010-2011 3/

B4. Net non-debt creating flows at historical average minus one standard deviation in 2010-2011 4/

B5. Combination of B1-B4 using one-half standard deviation shocks

B6. One-time 30 percent nominal depreciation relative to the baseline in $20105 /$

Memorandum item:

Grant element assumed on residual financing (i.e., financing required above baseline) 6

$\begin{array}{lllllllllll}30 & 30 & 30 & 30 & 30 & 30 & 30 & 30 & 30 & 30 & 30\end{array}$

Source: IMF staff projections and simulations.

1/ Variables include real GDP growth, growth of GDP deflator (in U.S. dollar terms), non-interest current account in percent of GDP, and non-debt creating flows.

2/ Assumes that the interest rate on new borrowing is by 2 percentage points higher than in the baseline., while grace and maturity periods are the same as in the baseline.

of GDP is assumed to return to its baseline level after the shock (implicitly assuming an offsetting adjustment

$4 /$ Includes official and private transfers and FDI.

$5 /$ Depreciation is defined as percentage decline in dollar/local currency rate, such that it never exceeds 100 percent.

6/ Applies to all stress scenarios except for A2 (less favorable financing) in which the terms on all new financing are as specified in footnote 2. 
Table 3. Democratic Republic of the Congo: Public Sector Debt Sustainability Framework, Baseline Scenario, 2008-29 (In percent of GDP, unless otherwise indicated)

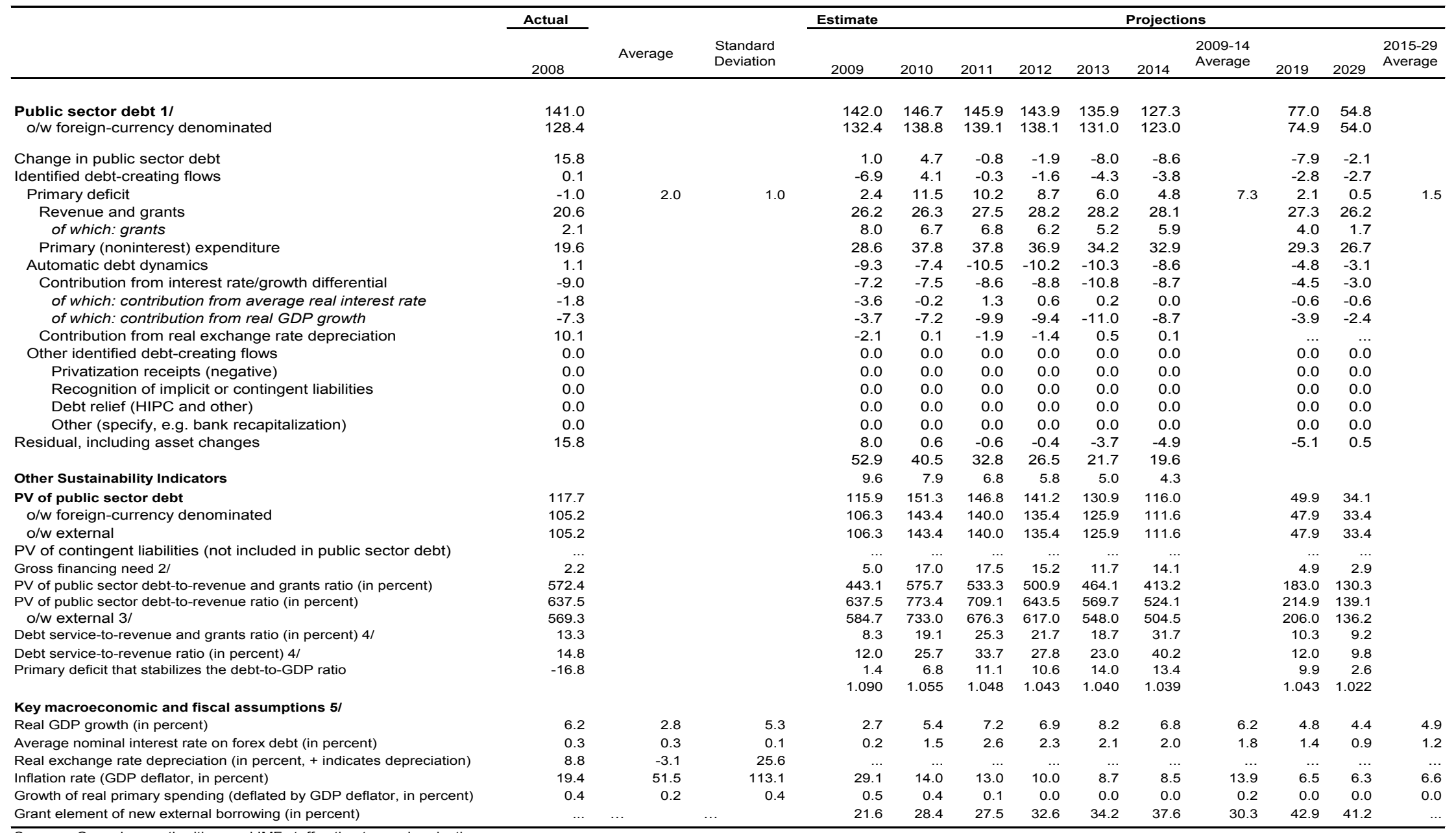

Sources: Congolese authorities; and IMF staff estimates and projections.

1/ Gross general government debt.

2/ Gross financing need is defined as the primary deficit plus debt service plus the stock of short-term debt at the end of the last period.

3/ Revenues excluding grants.

4/ Debt service is defined as the sum of interest and amortization of medium and long-term debt.

5/ Historical averages and standard deviations are generally derived over the past 10 years, subject to data availability. 
Table 4. Democratic Republic of the Congo: Sensitivity Analysis for Key Indicators of Public Debt 2009-29

\begin{tabular}{|c|c|c|c|c|c|c|c|c|}
\hline & \multicolumn{8}{|c|}{ Projections } \\
\hline & 2009 & 2010 & 2011 & 2012 & 2013 & 2014 & 2019 & 2029 \\
\hline \multicolumn{9}{|l|}{ PV of Debt-to-GDP Ratio } \\
\hline Baseline & 115.9 & 151.3 & 146.8 & 141.2 & 130.9 & 116.0 & 49.9 & 34.1 \\
\hline \multicolumn{9}{|l|}{ A. Alternative scenarios } \\
\hline A1. Real GDP growth and primary balance are at historical averages & 115.9 & 145.4 & 138.4 & 130.8 & 121.5 & 105.0 & 25.9 & 9.4 \\
\hline A2. Primary balance is unchanged from 2009 & 115.9 & 143.2 & 132.1 & 121.6 & 109.0 & 92.7 & 30.9 & 30.0 \\
\hline A3. Permanently lower GDP growth 1/ & 115.9 & 153.1 & 150.6 & 147.1 & 138.8 & 125.5 & 67.5 & 91.2 \\
\hline \multicolumn{9}{|l|}{ B. Bound tests } \\
\hline B1. Real GDP growth is at historical average minus one standard deviations in $2010-2011$ & 115.9 & 164.7 & 179.0 & 175.9 & 167.1 & 152.6 & 90.1 & 101.5 \\
\hline B2. Primary balance is at historical average minus one standard deviations in $2010-2011$ & 115.9 & 143.7 & 133.1 & 128.2 & 118.4 & 103.9 & 39.6 & 26.1 \\
\hline B3. Combination of B1-B2 using one half standard deviation shocks & 115.9 & 150.1 & 147.5 & 144.4 & 136.0 & 122.1 & 61.0 & 67.0 \\
\hline B4. One-time 30 percent real depreciation in 2010 & 115.9 & 219.0 & 208.5 & 197.8 & 183.7 & 163.3 & 81.3 & 62.2 \\
\hline B5. 10 percent of GDP increase in other debt-creating flows in 2010 & 115.9 & 160.2 & 155.3 & 149.4 & 138.7 & 123.5 & 56.4 & 39.1 \\
\hline
\end{tabular}

PV of Debt-to-Revenue Ratio 2/

Baseline

$\begin{array}{llllllll}443.1 & 575.7 & 533.3 & 500.9 & 464.1 & 413.2 & 183.0 & 130.3\end{array}$

\section{A. Alternative scenarios}

A1. Real GDP growth and primary balance are at historical averages

A2. Primary balance is unchanged from 2009

A3. Permanently lower GDP growth $1 /$

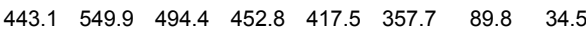

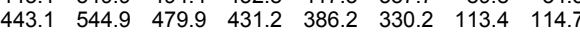

$\begin{array}{llllllll}443.1 & 581.1 & 544.2 & 518.0 & 487.9 & 442.1 & 243.3 & 343.0\end{array}$

\section{B. Bound tests}

B1. Real GDP growth is at historical average minus one standard deviations in 2010-2011

B2. Primary balance is at historical average minus one standard deviations in 2010-2011

B3. Combination of B1-B2 using one half standard deviation shocks

B4. One-time 30 percent real depreciation in 2010

B5. 10 percent of GDP increase in other debt-creating flows in 2010

$\begin{array}{lllllllr}443.1 & 614.3 & 621.7 & 599.0 & 572.5 & 523.1 & 321.7 & 383.0 \\ 443.1 & 546.9 & 483.6 & 454.5 & 419.8 & 370.3 & 145.1 & 99.7 \\ 443.1 & 563.7 & 519.6 & 498.4 & 471.3 & 423.7 & 219.8 & 253.9 \\ 443.1 & 833.3 & 757.7 & 701.6 & 651.0 & 581.7 & 298.2 & 237.5 \\ 443.1 & 609.5 & 564.2 & 529.8 & 491.7 & 440.0 & 206.7 & 149.3\end{array}$

Debt Service-to-Revenue Ratio 2l

Baseline

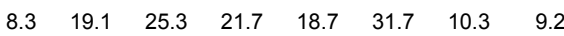

\section{A. Alternative scenarios}

A1. Real GDP growth and primary balance are at historical averages

A2. Primary balance is unchanged from 2009

A3. Permanently lower GDP growth 1/

$\begin{array}{rrrrrrrr}8.3 & 19.5 & 25.1 & 20.4 & 17.0 & 31.9 & 7.3 & -2.6 \\ 8.3 & 19.1 & 24.1 & 19.2 & 15.6 & 28.3 & 7.3 & 4.3 \\ 8.3 & 19.3 & 25.8 & 22.3 & 19.6 & 33.4 & 12.8 & 20.4\end{array}$

B. Bound tests

B1. Real GDP growth is at historical average minus one standard deviations in 2010-2011 B2. Primary balance is at historical average minus one standard deviations in 2010-2011 B3. Combination of B1-B2 using one half standard deviation shocks

B4. One-time 30 percent real depreciation in 2010

B5. 10 percent of GDP increase in other debt-creating flows in 2010

Debt Service-to-GDP Ratio

Baseline

$\begin{array}{rrrrrrrr}8.3 & 20.2 & 28.9 & 25.4 & 22.7 & 38.0 & 16.1 & 25.5 \\ 8.3 & 19.1 & 24.1 & 19.4 & 16.6 & 29.8 & 8.7 & 5.7 \\ 8.3 & 19.8 & 26.2 & 20.9 & 18.3 & 33.2 & 11.8 & 14.9 \\ 8.3 & 24.0 & 37.6 & 32.6 & 28.6 & 48.6 & 19.1 & 23.2 \\ 8.3 & 19.1 & 26.7 & 23.2 & 19.9 & 32.9 & 11.2 & 11.3\end{array}$

\section{A. Alternative scenarios}

A1. Real GDP growth and primary balance are at historical averages

A2. Primary balance is unchanged from 2006

A3. Permanently lower GDP growth 1/

$\begin{array}{rrrrrrrr}2.2 & 5.1 & 7.0 & 5.9 & 5.0 & 9.4 & 2.1 & -0.7 \\ 2.2 & 5.0 & 6.6 & 5.4 & 4.4 & 7.9 & 2.0 & 1.1 \\ 2.2 & 5.1 & 7.1 & 6.3 & 5.6 & 9.5 & 3.5 & 5.4\end{array}$

\section{B. Bound tests}

B1. Real GDP growth is at historical average minus one standard deviations in 2008-2009

B2. Primary balance is at historical average minus one standard deviations in 2008-2009

B3. Combination of B1-B2 using one half standard deviation shocks

B4. One-time 30 percent real depreciation in 2008

B5. 10 percent of GDP increase in other debt-creating flows in 2008 
INTERNATIONAL MONETARY FUND

THE DEMOCRATIC REPUBLIC OF THE CONGO

Staff Report for the 2009 Article IV Consultation, Request for a Three-Year Arrangement Under the Poverty Reduction and Growth Facility, and Request for Additional Interim Assistance Under the Enhanced Initiative for Heavily Indebted Poor Countries

\section{Informational Annex}

Prepared by the African Department

November 30, 2009

- $\quad$ Relations with the Fund. Describes financial and technical assistance from the Fund and provides information on the safeguards assessment and exchange rate system. Outstanding purchases and loans amounted to SDR 502.71 million (94.32 percent of quota) as of October 31, 2009.

- Joint Managerial Action Plan. Describes the IMF's and World Bank Group's work program.

- $\quad$ Statistical Issues. Provides assessment of data adequacy for surveillance and program monitoring purposes.

- Table of Common Indicators for Surveillance. Provides information on statistical data for surveillance purpose. 


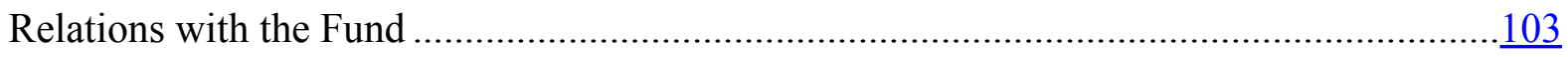

Relations with the World Bank Group....................................................................... 107

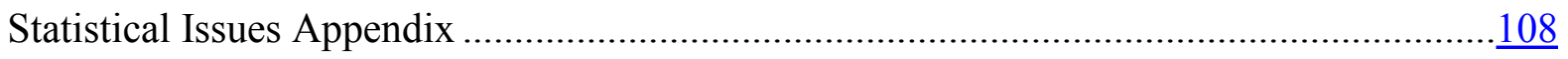

Table of Common Indicators Required for Surveillance...............................................109 


\section{DEMOCRATIC REPUBLIC OF THE CONGO \\ RELATIONS WITH THE FUND \\ (As of October 31, 2009)}

I. Membership Status: Joined September 28, 1963; Article VIII

II. General Resources Account:

Quota

Fund holdings of currency

III. SDR Department:

Net cumulative allocation

Holdings

IV. Outstanding Purchases and Loans:

ESF RAC loan

Poverty Reduction and Growth Facility (PRGF)

Arrangement

V. Latest Financial Arrangements:

Type

PRGF

Stand-By

SAF Approval Date June 12, 2002

Expiration

Date

March 31, 2006

June 9, 1989

May 15, 1987

June 8, 1990

May 14, 1990
Amount Approved
(SDR Million)

580.00

116.40

203.70
Percent of Quota

533.00

100.00

533.00

100.00

SDR Million

510.86

419.46

SDR Million

133.25

369.46
Percent of Quota

100.0

82.11

Percent of Quota

25.00

69.32

VI. Projected Payments to Fund:

(SDR million; based on current use of resources and present holdings of SDRs):

\begin{tabular}{lrrrrr} 
& \multicolumn{5}{c}{ Forthcoming } \\
\cline { 2 - 6 } & $\mathbf{2 0 0 9}$ & $\mathbf{2 0 1 0}$ & $\mathbf{2 0 1 1}$ & $\mathbf{2 0 1 2}$ & $\mathbf{2 0 1 3}$ \\
Principle & 42.00 & 105.35 & 110.69 & 68.69 & 24.03 \\
Charges/interest & 1.32 & 2.39 & 1.85 & 1.31 & 1.09 \\
Total & 43.32 & 107.75 & 112.55 & 70.01 & 25.12
\end{tabular}


VII. Implementation of HIPC Initiative:

I. Commitment of HIPC assistance

Enhanced Framework

Decision point date

July 2003

Assistance committed by all creditors (US\$ millions)

$6,311.00$

of which: IMF assistance (US\$ millions)

(SDR equivalent millions)

228.30

Completion point date

Floating

II. Disbursement of IMF assistance (SDR millions)

Assistance disbursed to the member $\quad 3.39$

Interim assistance

3.39

Completion point balance

Additional disbursement of interest income

Total disbursement

3.39

VIII. Implementation of MDRI Assistance: Not applicable

IX. Exchange Rate Arrangement:

The currency of the Democratic Republic of the Congo is the Congo franc which, since May 26, 2001, has been freely floating. On November 25, 2009, the rate was US\$1=CF 905.

Effective February 10, 2003, the DRC accepted the obligations of Article VIII, Sections 2 (a), 3, and 4, of the Fund's Articles of Agreement; however, the DRC maintains measures that give rise to one restrictions and one multiple currency practice subject to Fund approval. The exchange restrictions involves an outstanding net debt position against other contracting members under the inoperative regional payments agreement with the Economic Community of the Great Lakes Countries. The multiple currency practice relates to a fixed exchange rate set quarterly applying to transactions through the bilateral payments agreement with Zimbabwe.

\section{Last Article IV Consultation:}

(a) Consultations with the Democratic Republic of the Congo are held in accordance with the provisions of the decision on consultation cycles approved on July 15, 2002.

(b) The last Article IV consultation was concluded by the Executive Board on September 5, 2007. 


\section{Safeguards Assessment:}

A safeguards assessment of the central bank (BCC) was completed on December 24, 2002, and updated in January 2008. Both initial and updated assessments concluded that there may be substantial risks of misreporting or misuse due to vulnerabilities in the external audit mechanism, financial reporting, and system of internal controls. Staff findings, recommendations related to program conditionality, and other recommendations may be found in IMF Country Report No. 03/161 and IMF Country Report No. 07/328. In the context of the proposed PRGF, an update safeguards assessment of the Banque Centrale du Congo (BCC) is currently in progress.

\section{Technical Assistance:}

\section{STA}

FY 2004 - Multisector statistics

FY 2010 - Monetary statistics

\section{MCM}

\section{Capacity building assistance to the central bank}

Strengthening capacity in Anti-Money Laundering/Combating Financial Terrorism (AML/CFT)

FY 2005 - One short visit

FY 2006 - Two short visits

Strengthening capacity in monetary policy implementation, exchange operations, and liquidity management

FY 2005 - Three short visits

FY 2006 - Two short visits

FY 2007 - Seven short visits

FY 2008 - Three short visits

FY 2009 - One short visit

Restructuring and reorganization of the central bank

FY 2009 - One short visit

FY 2010 - One short visit

Strengthening capacity in banking supervision and financial sector development

FY 2005 - One short visit on bank supervision and regulation

FY 2008 - Two short visits on bank supervision and regulation

FY 2010 - Three short visits on bank supervision and regulation

FY 2006 - One short visit on internal audit

FY 2008 - One short visit on internal audit

FY 2007 - Action plan for developing the financial system

FY 2008 - One short visit on TA assessment 


\section{FAD}

Public financial management

FY 2007 - Short visit

FY 2009 - Short visit

Revenue administration

FY 2004 - Two short visits

FY 2005 - One short visit

FY 2006 - One short visit

FY 2007 - Short visit

FY 2007 - short visit

FY 2009 - short visit

Fiscal decentralization

FY 2005 - Short visit

XIII. Long-term Resident Experts:

Expenditure management

Customs administration

Tax administration

Advisor to Governor of BCC

Capacity building of BCC

Statistics
Mr. Catalan

Mr. Nguenang

Mr. Bremeersch

Mr. Fossat

Mr. Nyssens

Mr. d'Ambrières

Mr. Pauwels

Mr. Métreau

Mr. Gbossa
Jan. 2002-Jun. 2004

Since Sept. 2004

Jan. 2002-Jan. 2004

Sept 2005

Jan. 2004-Jan. 2005

Since Feb. 25, 2006

Since Sept. 2004

Oct. 2004

Dec. 2005-08

XIV. Resident Representative: Mr. Jahjah assumed his duties as Resident Representative in January 2009. 


\section{Democratic Republic of the Congo: Relations with the World Bank Group Joint Managerial Action Plan (JMAP) (As of October 31, 2009)}

1. The IMF and World Bank Democratic Republic of Congo teams discussed a joint managerial action plan under this initiative.

2. The World Bank's work program entails moving forward in FY10 with a Development Policy Operation (DPO) to provide US\$100 million in budget support, a Diagnostic Trade Integration Study (DTIS) and a Country Economic Memorandum (CEM) that will focus on constraints to growth.

3. The IMF's work program entails a new PRGF arrangement.

4. The staffs are working together to deliver (a) a JSAN of the implementation of the existing PRSP and (b) the HIPC completion point document.

5. Both institutions are supporting an FSAP that will take place during FY11.

\begin{tabular}{|c|c|c|c|}
\hline Title & Products & Provisional timing of missions & Actual or Expected delivery date \\
\hline \multicolumn{4}{|c|}{ A. Mutual information on relevant work program } \\
\hline \multirow[t]{6}{*}{$\begin{array}{l}\text { World Bank work program in } \\
\text { the next } 12 \text { months }\end{array}$} & $\begin{array}{l}\text { Extension of Interim HIPC debt } \\
\text { relief }\end{array}$ & None & October 2009 \\
\hline & $\begin{array}{l}\text { Development Policy Operation } \\
\text { that may provide }\end{array}$ & $\begin{array}{l}\text { September 2009-November } \\
2009\end{array}$ & March 2010 \\
\hline & $\begin{array}{l}\text { US } \$ 100 \text { million in budget } \\
\text { support }\end{array}$ & February 2010 & \\
\hline & $\begin{array}{l}\text { Diagnostic Trade Integration } \\
\text { Study }\end{array}$ & October 2009 & March 2010 \\
\hline & Country Economic & October 2009 & September 2010 \\
\hline & Memorandum & July 2009 & \\
\hline \multirow{2}{*}{$\begin{array}{l}\text { IMF work program in the next } \\
12 \text { months }\end{array}$} & Article IV and PRGF program & - & December 2009 \\
\hline & First review of PRGF & February/March 2010 & June 2010 \\
\hline \multicolumn{4}{|c|}{ B. Requests for work program inputs } \\
\hline Fund request to Bank & $\begin{array}{l}\text { Provide comments on } \\
\text { implications of sector-specific } \\
\text { issues, including developments } \\
\text { in the mining, energy, telecom } \\
\text { and agricultural sectors. }\end{array}$ & None & FY 10 \\
\hline Bank request to Fund & $\begin{array}{l}\text { Macroeconomic scenarios for } \\
\text { HIPC Interim Debt Relief, } \\
\text { DPO, DTIS and CEM }\end{array}$ & None & FY 10 \\
\hline \multicolumn{4}{|c|}{ C. Agreement on joint products and missions } \\
\hline \multirow{4}{*}{$\begin{array}{l}\text { Joint products in the next } \\
12 \text { months }\end{array}$} & JSAN of PRSP progress report & February/March 2010 & June 2010 \\
\hline & $\begin{array}{l}\text { HIPC/MDRI completion point } \\
\text { document }\end{array}$ & March 2010 & June 2010 \\
\hline & $\begin{array}{l}\text { Public Finance Management } \\
\text { (PFM) technical assistance }\end{array}$ & FY10-11 & FY10-11 \\
\hline & $\begin{array}{l}\text { Banking sector supervision } \\
\text { technical assistance }\end{array}$ & FY10-11 & FY10-11 \\
\hline
\end{tabular}




\section{Democratic Republic of the Congo: Statistical Issues Appendix (As of October 31, 2009)}

\section{Assessment of Data Adequacy for Surveillance}

General: Data provision to the Fund is broadly adequate for surveillance and program monitoring purposes, but upgrades to the national accounts, balance of payments, and government finance statistics would be helpful. In spite of difficulties, the authorities have continued to produce an array of economic and financial statistics, most of which are published in the annual report of the Central Bank of the Congo (BCC) or its monthly statistical bulletin.

National accounts: Annual national accounts are compiled in constant and current prices by the Directorate of Research of the BCC and published yearly. The compilation methodology conforms to the System of National Accounts 1968 (1968 SNA) and is based on the balance sheets of enterprises and surveys of public and semipublic enterprises and agencies, most of which date from the late 1980s. The activities of the traditional sector (including the informal sector) are estimated with extrapolation techniques using industry-specific data that are outdated. As a result, GDP is likely to be underestimated.

With assistance from AFRITAC, work on the national accounts is underway to update the base year to 2005, incorporate the results of a recent 1-2-3 household survey, as well as implement the methodological recommendations of the 1993 SNA. While outputs of this work remain to be validated and officially published, as expected, the 2005 GDP estimates show a considerable upward revision compared to previously published estimates.

Price statistics: Consumer price indices are calculated for Kinshasa by the BCC, the National Statistics Institute (INS), the Institute of Economic and Social Research (IESR), and the Economics Section of the U.S. Embassy in Kinshasa. The IESR also calculates a monthly consumer price index for the Lubumbashi market. The household surveys on which these calculations are based date back to the late 1980s; they need to be updated to account for changes in household consumption patterns and demographic shifts, while geographical coverage should be expanded.

Government finance statistics: The BCC produces aggregated monthly statistics on a cash basis based on its own accounting for the government cash operations. The treasury produces two sets of monthly statistics based on its own records: one relates to the transactions executed through the BCC, the other set attempts to consolidate operations through commercial bank accounts and off-budget operations. These statistics do not rely on an integrated double-entry public accounting system and do not provide sufficient details about the nature of expenditures owing to problems in the expenditure chain. However, the treasury has started to produce quarterly expenditure data reports by ministry and institution.

The current work on tax administration and expenditure control is expected to improve the quality and timeliness of fiscal statistics. In parallel with technical assistance on public expenditure management, a STA multisector statistics advisor has been advising on government finance statistics, producing improvements in the quality of the statistics. Furthermore, adoption of a simplified double-entry bookkeeping system should significantly improve the availability of data on government financial operations.

In 2003 the DRC reported annual data up to 2002 for the IMF's Government Finance Statistics Yearbook in the format of the Government Finance Statistics Manual 2001, but has not reported subsequently.

Monetary statistics: The BCC Directorate of Research regularly produces timely monetary statistics. These statistics are now generally reliable, but some problems remain with the classification of accounts.

Since STA money and banking statistics mission of June 2000, BCC reporting of data for publication in the IMF's International Financial Statistics (IFS) has improved and monetary data are currently reported on time. A 2003 multisector statistics mission recommended a new chart of accounts for the BCC and other deposit-taking institutions, including the information needed for improved sectorization of economic units and classification of financial assets.

Balance of payments: Annual balance of payments statistics are compiled with information on the exports and imports of large public and semipublic enterprises, $\mathrm{BCC}$ payment records, and a survey of the foreign operations of residents. The estimates also take account of information on the informal sector and foreign aid flows provided by the World Bank and the local UN Development Program (UNDP) office, which collects the data from the European Union, embassies, and nongovernmental organizations. However, the 2003 multisector mission found that, due to computer problems, data have not been processed since 1999, so in effect balance of payments statistics are compiled using data from different sources, supplemented by estimates. The mission suggested that the BCC implement a system of quarterly surveys of corporations that are authorized to hold accounts overseas. The mission also recommended reinvigoration of a working group comprising staff of the customs and other agencies to prepare quarterly foreign trade data and take measures to improve data on services and transfers. No external sector data are reported to STA for publication.

\section{Data Standards and Quality}

Since April 24, 2004, the Democratic Republic of the Congo participates in the general Data Dissemination System (GDDS). The metadata needs to be updated. 


\begin{tabular}{|l|r|r|r|r|c|}
\hline \multicolumn{5}{|c|}{ The Democratic Republic of the Congo: Table of Common Indicators Required for Surveillance } \\
(As of November 25, 2009)
\end{tabular}

${ }^{1}$ Any reserves assets that are pledged or otherwise encumbered should be specified separately. Also, data should comprise short-term liabilities linked to a foreign currency but settled by other means as well as the national values of financial derivatives to pay and receive foreign currency, including those linked to a foreign curreny but settled by other means.

${ }^{2}$ Both market-based and officially-determined, including discount rates, money markets rates, rates on treasury bills, notes and bonds.

${ }^{3}$ Foreign, domestic bank, and domestic nonbank financing.

${ }^{4}$ Including currency and maturity composition.

${ }^{5}$ Includes external gross financial asset and libility positions vis-à-vis nonresidents.

${ }^{6}$ Daily(D), Weekly (W), Monthly (M), Quarterly (Q), Irregular (I), Not available (NA). 
Press Release No. 09/455

International Monetary Fund

FOR IMMEDIATE RELEASE

Washington, D.C. 20431 USA

December 11, 2009

\section{IMF Executive Board Approves US\$551 Million PRGF Arrangement for the Democratic Republic of the Congo and US\$73 Million in Interim HIPC Assistance}

The Executive Board of the International Monetary Fund (IMF) today approved a three-year, SDR 346.45 million (about US\$551.45 million) arrangement for the Democratic Republic of the Congo under the Poverty Reduction and Growth Facility (PRGF). The Executive Board also approved additional interim assistance of SDR 45.66 million (about US\$72.68 million) under the enhanced Heavily Indebted Poor Countries (HIPC) Initiative to reduce the DRC's debt service payments to the IMF.

Satisfactory completion of the first review of the government's PRGF-supported economic program is a key requirement for the DRC to reach the completion point under the enhanced HIPC Initiative and to benefit from the Multilateral Debt Relief Initiative (MDRI), which together could result in relief from the vast majority of the country's external debt. Other requirements for the HIPC completion point are 12 months of satisfactory implementation of the government's Poverty Reduction and Growth Strategy (PRGS) and other measures--including in social areas such as healthcare and education--which were agreed when the DRC reached its HIPC Decision Point in July 2003.

Following the Executive Board's discussion of the Democratic Republic of the Congo, Mr. John Lipsky, First Deputy Managing Director and Acting Chair, said:

"The Democratic Republic of the Congo (DRC) has made important socioeconomic progress since 2001, demonstrated by the political transition to a democratically-elected government, robust rates of economic growth, and the taming of hyperinflation. Nonetheless, socioeconomic conditions remain poor; the country's infrastructure is dilapidated; and the country's external debt is unsustainable. The global economic crisis has further aggravated these conditions. 
"The new three-year PRGF arrangement will support the authorities' implementation of their poverty reduction and growth strategy and economic reform program. The key priorities are to generate strong economic growth, reduce inflation to single digits, strengthen public financial management, achieve debt sustainability, and accelerate structural reforms.

"Prudent and credible fiscal policies are key to addressing fiscal dominance over monetary policy and making progress toward fiscal sustainability. The authorities aim to bolster domestic revenue, strengthen expenditure management, and implement a prudent wage policy to support fiscal consolidation while expanding priority spending programs. The authorities are committed to avoiding recourse to bank financing over the medium term.

"Monetary policy will continue to focus on reducing inflation. The central bank aims to enhance the effectiveness of monetary policy by improving its liquidity forecasting capacity and strengthening coordination with the Treasury. Completion of the restructuring and recapitalization of the central bank is important to enhance the central bank's credibility and independence. The central bank's supervision of the commercial banks also needs to be strengthened.

“The DRC's flexible exchange rate regime has served the country well. The central bank will limit intervention in the foreign exchange market to smoothing short-term volatility and achieving its gross official reserve target.

"Strong and sustained economic growth requires implementation of key structural reforms. The authorities will focus on public enterprise reform, improving governance, and streamlining the business regulatory environment.

"Given the DRC's high debt burden, the authorities recognize the need for prudent debt management. They aim to meet the enhanced HIPC Initiative requirements and triggers to reach the completion point and receive debt relief under the Multilateral Debt Relief Initiative as early as possible.

Continued highly concessional assistance from the donor community will be necessary to support the authorities' reform efforts."

Links to interviews with Mr. Brian Ames, IMF Mission Chief:

Audio: $\quad$ http://www.imf.org/external/mmedia/view.asp?eventID=1696

Video (English) $\quad$ http://www.imf.org/external/mmedia/view.asp?eventID $=1673$

Video (French): $\quad$ http://www.imf.org/external/mmedia/view.asp?eventID=1674 


\section{Recent Economic Developments}

Socioeconomic conditions in the DRC remain dire following a decade-long conflict that has had enormous human costs and resulted in widespread destruction of the country's social and economic infrastructure. Although rich in natural resources, the country's per capita income and human development indicators remain among the lowest in Africa.

A sharp decline in world commodity prices in late 2008 slowed economic growth in 2009 to a projected 2.7 percent, while the escalation of conflict in the eastern provinces led to higher security spending, increased government borrowing from the central bank, and an upsurge in inflation and depreciation of the exchange rate during the first quarter of 2009. The Congolese franc depreciated by 35 percent against the U.S. dollar between December 2008 and September 2009. Reserves declined to a historically low level of US $\$ 30$ million in February 2009 before rising to US\$894 million at end-September following the disbursement of emergency assistance from the Fund and other development partners, the arrival of the first tranche of the Sino-Congolese Cooperation Agreement (SCCA) signing bonus, and the IMF's general and special SDR allocations (SDR 424.5 million).

The DRC is in debt distress with an external debt stock of about US\$13.1 billion and debt service amounting to about one-fourth of total expenditure. At the end of 2008, publicly contracted or guaranteed external debt was an estimated 93 percent of GDP, 150 percent of exports, and 502 percent of government revenue, excluding foreign aid. The DRC reached the decision point of the enhanced HIPC Initiative in July 2003. It is current on repayments to multilateral financial institutions including the IMF, but continues to accumulate arrears vis-à-vis bilateral and commercial creditors. IMF staff received financing assurances in November 2009 regarding the rescheduling of the DRC's debts to the Paris Club of sovereign bilateral creditors.

\section{Program Summary}

The Congolese Authorities' program for the three years to June 2012 includes the following targets: (i) average real GDP growth of 5.5 percent; (ii) end-period inflation rate of 9 percent by 2012;

(iii) gross reserves equivalent to 10 weeks of nonaid imports by 2012; and (iv) the external current account deficit (including grants) limited to an average of 25 percent of GDP.

The program includes structural reforms that focus on:

- Improving domestic revenue mobilization by expanding the tax base and improving its management

- Strengthening public financial management through improved budget preparation, execution and accountability 
- Reforming the central bank reform, with a focus on restoring the independence of the Banque Centrale du Congo and strengthening its supervisory capacity with technical assistance from the IMF's Monetary and Capital Markets department

- Developing the private sector, including the reform of public enterprises, streamlining government regulations, protecting foreign investment and improving transparency in natural resource management.

The authorities' 2006-08 PRGS, which has been extended through 2010 and endorsed by IMF staff, is based on the five pillars: promotion of peace and good governance; consolidation of macroeconomic stability and promotion of economic growth; improvement in access to social services; the fight against HIV/AIDS; and promotion of a dynamic community.

The DRC became a member of the IMF on September 28, 1963 and has a quota in the Fund of SDR 533 million. The DRC's previous PRGF arrangement, approved in June 2002 in the sum of SDR 580 million (US\$924 million -- see Press Release No. 02/27), was almost fully drawn (SDR 553.47 million) before it expired in March 2006 without completion of the sixth review. IMF staff have subsequently monitored the Congolese authorities' economic program through a series of staff monitored programs. On March 12, 2009, the Executive Board approved a disbursement in an amount equivalent to SDR 133.25 million (about US\$212 million) to the Democratic Republic of the Congo under the Rapid-Access Component of the Exogenous Shocks Facility (RAC-ESF - see Press Release No. 09/74). 
114

Table 1. Democratic Republic of the Congo: Selected Economic and Financial Indicators, 2007-14

\begin{tabular}{|c|c|c|c|c|c|c|c|c|}
\hline & 2007 & $\underline{2008}$ & $\underline{2009}$ & 2010 & 2011 & 2012 & 2013 & 2014 \\
\hline & & Prel. & Est. & \multicolumn{5}{|c|}{ Projections } \\
\hline & \multicolumn{8}{|c|}{ (Annual percentage change; unless otherwise indicated) } \\
\hline \multicolumn{9}{|l|}{ GDP and prices } \\
\hline Real GDP & 6.3 & 6.2 & 2.7 & 5.4 & 7.3 & 6.8 & 8.1 & 7.0 \\
\hline GDP deflator & 17.8 & 19.4 & 30.3 & 23.4 & 12.8 & 9.8 & 8.3 & 8.4 \\
\hline Consumer prices, period average & 16.7 & 18.0 & 45.0 & 24.7 & 13.5 & 10.5 & 9.0 & 8.8 \\
\hline Consumer prices, end-of-period & 10.0 & 27.6 & 48.7 & 15.0 & 12.0 & 9.0 & 9.0 & 8.5 \\
\hline \multicolumn{9}{|l|}{ External sector } \\
\hline Exports, f.o.b. (U.S.Dollars) & 109.6 & 7.2 & -42.5 & 18.3 & 27.8 & 13.2 & 17.2 & 16.4 \\
\hline Imports, f.o.b. (U.S.Dollars) & 81.8 & 27.6 & -21.7 & 21.6 & 26.1 & 0.5 & 4.5 & 6.8 \\
\hline Export volume & 75.0 & -3.9 & 5.4 & 9.4 & 23.9 & 11.8 & 16.5 & 13.9 \\
\hline Import volume & 69.0 & 15.8 & -11.9 & 17.0 & 23.4 & -1.4 & 2.5 & 4.9 \\
\hline Terms of trade & 11.3 & 1.1 & -38.6 & 4.1 & 0.9 & -0.8 & -1.4 & 0.3 \\
\hline Nominal effective exchange rate ${ }^{1}$ & -15.5 & -11.7 & $\ldots$ & $\ldots$ & $\ldots$ & $\ldots$ & $\ldots$ & $\ldots$ \\
\hline Real effective exchange rate ${ }^{1}$ & -3.1 & -0.7 & $\ldots$ & $\ldots$ & $\ldots$ & $\ldots$ & $\ldots$ & $\ldots$ \\
\hline \multicolumn{9}{|c|}{$\begin{array}{c}\text { (Annual change in percent of beginning-of-period broad money; } \\
\text { unless otherwise indicated) }\end{array}$} \\
\hline \multicolumn{9}{|l|}{ Money and credit } \\
\hline Broad money & 49.5 & 55.7 & 33.8 & 27.0 & $\ldots$ & $\ldots$ & $\ldots$ & $\ldots$ \\
\hline Net foreign assets & 30.8 & -6.0 & -6.9 & 38.6 & $\ldots$ & $\ldots$ & $\ldots$ & $\ldots$ \\
\hline Net domestic assets & 20.2 & 65.6 & 41.6 & -11.2 & $\ldots$ & $\ldots$ & $\ldots$ & $\ldots$ \\
\hline Domestic credit & 29.5 & 54.7 & 15.6 & 13.8 & $\ldots$ & $\ldots$ & $\ldots$ & $\ldots$ \\
\hline \multicolumn{9}{|l|}{ Of which: } \\
\hline Net credit to government & 10.3 & 11.3 & -10.6 & 0.0 & \multirow{2}{*}{$\begin{array}{l}\cdots \\
\cdots\end{array}$} & $\ldots$ & \multirow{2}{*}{$\begin{array}{l}\ldots \\
\ldots\end{array}$} & \multirow{2}{*}{$\begin{array}{l}\cdots \\
\cdots\end{array}$} \\
\hline $\begin{array}{l}\text { Credit to the private sector (annual } \\
\text { percent change) }\end{array}$ & 73.6 & 141.9 & 55.5 & 25.4 & & $\ldots$ & & \\
\hline & \multicolumn{8}{|c|}{ (Percent of GDP; unless otherwise indicated) } \\
\hline \multicolumn{9}{|l|}{ Central government finance } \\
\hline Total government revenue & 14.8 & 18.5 & 18.0 & 17.9 & 19.0 & 20.2 & 20.8 & 21.2 \\
\hline $\begin{array}{l}\text { Excluding signing bonus from the Sino- } \\
\text { Congolese Cooperation Agreement }\end{array}$ & 14.8 & 18.5 & 15.7 & 17.9 & 19.0 & 20.2 & 20.8 & 21.2 \\
\hline Grants & 1.5 & 1.9 & 7.9 & 6.1 & 6.2 & 5.7 & 4.8 & 4.3 \\
\hline Total government expenditure $^{2}$ & 18.8 & 22.7 & 29.1 & 36.2 & 35.3 & 34.6 & 32.1 & 30.8 \\
\hline Underlying fiscal balance (cash basis) & 0.8 & 0.9 & -1.9 & -0.5 & -0.6 & -0.4 & 0.4 & 0.4 \\
\hline Overall fiscal balance (payment order & & & & & & & & \\
\hline basis, incl. grants) & -2.5 & -2.3 & -3.2 & -12.2 & -10.1 & -8.7 & -6.4 & -5.3 \\
\hline Overall fiscal balance (cash basis, incl. grants) & -3.1 & -3.1 & -4.8 & -12.7 & -10.8 & -9.2 & -6.2 & -4.9 \\
\hline \multicolumn{9}{|l|}{ Investment and saving } \\
\hline Gross national saving & 16.7 & 6.1 & 7.0 & 5.8 & 5.6 & 7.4 & 11.2 & 14.4 \\
\hline Government & -1.0 & -0.5 & 1.0 & -0.1 & 1.8 & 2.9 & 3.8 & 4.2 \\
\hline Nongovernment & 17.7 & 6.5 & 6.0 & 5.9 & 3.8 & 4.4 & 7.4 & 10.2 \\
\hline Investment & 18.3 & 22.0 & 23.4 & 33.5 & 37.3 & 32.5 & 30.8 & 30.0 \\
\hline Government $^{3}$ & 2.4 & 3.7 & 9.3 & 18.0 & 17.9 & 17.1 & 15.1 & 13.8 \\
\hline Nongovernment & 15.9 & 18.2 & 14.1 & 15.5 & 19.4 & 15.4 & 15.7 & 16.2 \\
\hline \multicolumn{9}{|l|}{ Balance of payments } \\
\hline Exports of goods and services & 65.5 & 61.3 & 41.8 & 42.7 & 49.0 & 50.8 & 53.9 & 57.2 \\
\hline Imports of goods and services & 68.9 & 76.4 & 66.4 & 71.1 & 81.1 & 76.0 & 73.0 & 71.8 \\
\hline Current account balance, incl. transfers & -1.5 & -15.9 & -16.4 & -27.7 & -31.7 & -25.1 & -19.6 & -15.6 \\
\hline Current account balance, excl. transfers & -9.0 & -24.7 & -30.4 & -34.4 & -37.7 & -30.7 & -24.6 & -20.2 \\
\hline $\begin{array}{l}\text { Current account balance, incl. transfers, after } \\
\text { debt relief }\end{array}$ & 3.0 & -12.3 & -13.0 & -24.1 & -27.3 & -21.6 & -16.8 & -13.4 \\
\hline $\begin{array}{l}\text { Gross official reserves (weeks of nonaid- } \\
\text { related imports of goods and services) }\end{array}$ & 1.5 & 1.0 & 9.9 & 8.7 & 9.2 & 9.9 & 11.5 & 11.5 \\
\hline
\end{tabular}


115

Table 1. Democratic Republic of the Congo: Selected Economic and Financial Indicators, 2007-14 (concluded)

External public debt

Total stock, including IMF ${ }^{4}$

Net present value (NPV) of debt ${ }^{5}$

NPV of debt (percent of exports of goods and services) ${ }^{5}$

Scheduled debt service

Percent of exports of goods and services

Percent of government revenue

(Millions of U.S. dollars; unless otherwise indicated)

Exchange rate, (Congo franc per U.S.

dollar)

Period average $\quad 516.0$

End-of-period 503.0

$\begin{array}{rrrrrrrr}13,425 & 13,149 & 12,785 & 4,329 & 5,386 & 6,434 & 6,882 & 7,761 \\ 10,820 & 10,729 & 10,603 & 4,106 & 5,255 & 6,248 & 6,599 & 7,326 \\ & & & & & & & \\ 264.5 & 189.7 & 175.5 & 73.8 & 98.9 & 100.8 & 90.9 & 88.0 \\ 739.6 & 891.2 & 815.5 & 252.1 & 346.6 & 430.4 & 355.7 & 350.8 \\ 11.3 & 14.0 & 18.2 & 4.9 & 5.5 & 6.0 & 4.3 & 3.7 \\ 45.6 & 37.8 & 29.4 & 8.8 & 10.6 & 11.8 & 9.0 & 8.2\end{array}$

$\begin{array}{llllllll}16.0 & 563 & \ldots & \ldots & \ldots & \ldots & \ldots & \ldots\end{array}$

Memorandum item:

Nominal GDP (billions of Congo

francs)

$5,148 \quad 6,526$

8,729

$11,361 \quad 13,746$

$16,117 \quad 18,869$

Sources: Congolese authorities; and IMF staff estimates and projections.

${ }^{1}$ Change in annual average. Minus sign indicates depreciation.

${ }^{2}$ Includes interest due before debt relief and expenditure financed by HIPC resources.

${ }^{3}$ Includes investment financed by resources released under the enhanced HIPC Initiative.

${ }^{4}$ End-of-period debt stock includes most of London Club debt (some US\$1.2 billion in 2008), which is expected to be bought back with deep discount grants from IDA, and accumulated arrears.

${ }_{5}^{5}$ Estimates and projections are based on the 2009 DSA and after HIPC Initiative interim relief assistance. Includes assistance beyond the terms of the enhanced HIPC Initiative granted by some Paris Club creditors. Exports are on a three-year backward moving average; projections assume DRC reaches the HIPC completion point during the first half of 2010. 


\section{Statement by Mr. Rutayisire, Executive Director Democratic Republic of the Congo December 11, 2009}

On behalf of my Congolese authorities, I would like to thank Management and staff for maintaining dialogue with the Democratic Republic of the Congo (DRC) throughout the years, amid difficult circumstances. In particular, I thank the Managing Director for his fruitful visit to Kinshasa in May 2009 which contributed to set the way forward in the relations between the DRC and the IMF. I would also like to commend staff for their quality advice and technical assistance to my Congolese authorities and, notably, for the constructive policy discussions held during their visits to DRC in the context of the 2009 Article IV consultation and program negotiations.

The Democratic Republic of the Congo has come a long way from a period of civil conflicts and macroeconomic instability. The process of reconciliation and peace embarked upon in 2001 resulted in encouraging economic results supported by the international community, notably the IMF. The overall implementation of the 2002-06 PRGF arrangement and subsequent staff-monitored programs (SMPs), while not always smoothly implemented due to capacity constraints, repeated conflicts and external shocks, testify of the authorities' willingness and ability to pursue sound macroeconomic policies and structural reforms.

To address the capacity constraints and other perceived risks to their program, my Congolese authorities have, in agreement with Fund staff, designed a series of reforms, notably to enhance public financial management and strengthen the central bank through restructuring and recapitalization. They have already put in place institutional committees at both the political and technical levels, with the view to enhance the government's program coordination, monitoring and evaluation capacity. Their structural reform agenda also includes boosting domestic revenue mobilization, promoting private sector development and diversifying the economy, with the aim to enhance the economy's capacity to better weather exogenous shocks. In particular, improving the business climate is at the core of the authorities' strategy to revitalize the private sector, and this objective has been forcefully reasserted at the highest level recently. As for the risks related to a reemergence of conflicts, my authorities are pushing ahead their peace efforts, as evidenced by an agreement reached early this year with rebel groups in the eastern provinces and consolidation of improved relations with neighboring countries, including at the diplomatic level. My authorities also count on increased financial and technical support of the international community, which are essential to sustaining their actions.

My authorities are fully aware that their reconstruction and development efforts are predicated on their attracting direct foreign investments and allocating considerable resources to infrastructure and the priority sectors of education, health and agriculture. These in turn depend on the country benefiting from significant debt relief under the HIPC Initiative and the MDRI given that the country is in debt distress. To this vein, my authorities are fully committed to meeting the HIPC trigger points set forth at the decision point, pursuing their poverty reduction efforts through the implementation of the country's updated Poverty Reduction and Growth Strategy (PRGS), and implementing their agenda of short- and medium-term economic policies and reforms laid out in their Memorandum of Economic and Financial Policies (MEFP). 
Despite the challenging external environment and domestic conditions, the performance of the most recent SMP has been satisfactory. All prior actions towards the PRGF-supported program and indicative targets through end-September were observed, while implementation of the structural reform agenda remains on track. These prior actions include measures to strengthen revenue mobilization; promote development of the private sector through identifying government arrears and adopting a plan to eliminate them; make operational the national financial intelligence unit; amend the Sino-Congolese cooperation agreement with the aim of meeting the concessionality and debt sustainability requirements; set the initial stage to reinforce program monitoring and control via audits of the PRGF criteria; and making institutional steps to modernize the central bank and improve its monetary policy design and banking supervision.

Based on these achievements and demonstrated commitment to sound policies and reforms going forward, my DRC authorities request Fund's financial support under a three-year PRGF arrangement with an access level of 65 percent of quota, adequate to meet the anticipated financing gap over 2009-12. They also request an additional interim assistance under the HIPC Initiative.

\section{Recent Economic Developments}

Against the backdrop of escalated conflict in 2008, consequent urgent spending pressures, global financial crisis and supply bottlenecks, economic activity in 2008 and 2009 fell short of the initial projections, reaching 6 percent and 2.7 percent respectively. Inflation has been on the rise in recent months, reaching more than 50 percent on an annual basis at end-October 2009 due to the aforementioned budget pressures and consequent government borrowing from the central bank, Banque Centrale du Congo (BCC). Moreover, the global financial crisis has contributed to wide the current account deficit, while the Congolese franc depreciated significantly against the U.S. dollar throughout the period of December 2008 to November 2009.

The fragile state of the banking sector, as evidenced by the capital adequacy, liquidity and net foreign exchange position ratios as well as the elevated foreign currency risk, is being addressed notably through tightening and enforcing prudential regulations and strengthening on-site and off-site banking supervision. The BCC is implementing this comprehensive strategy with technical assistance of the Fund and the World Bank. It is also taking steps to restructure one large bank.

Despite the recent difficult environment, the medium-term outlook remains bright, with growth projections of about 6 percent annually over 2010-12, owing to higher public and private investment and a recovery in the mining sector; declining inflation down to single digit by 2012 and significant capital and financial flows linked to large infrastructure and mining projects. My DRC authorities have, in close collaboration with Fund staff, designed a medium-term program underpinned by conservative assumptions and aiming at realistic objectives.

\section{The Medium-term Economic Program}

The design of DRC's 2009-12 economic program has built on the recommendations from the 2009 Article IV consultation which itself draws from the 2007 Article IV consultation. My authorities broadly agree with Fund staff recommendations aimed at fostering sustained and high growth, enhancing external sustainability and strengthening institutional capacity for better economic 
management. In some instances, the authorities are taking steps towards meeting these objectives, notably pushing forward DRC participation in OHADA to help simplify procedures for business registration and strengthen legal protection for foreign investment and amending an important cooperation agreement in the mining and infrastructure sectors to minimize risks of external debt distress.

\section{Fiscal policy}

The medium-term fiscal policy is geared at preventing government recourse to central bank financing. The authorities intend to reach a domestic fiscal deficit of 0.4 percent of GDP in 2012. On the revenue side, efforts will aim at mobilizing greater domestic revenue through the implementation of an action plan to enhance the efficiency of revenue collection and expand the tax base via the introduction of a single rate VAT; measures to rationalize tax exemptions and streamline duties and taxes; modernize the customs and tax administrations; as well as efforts to increase the contribution of the mining sector to domestic revenue by strengthening the Large Taxpayers Office responsible, within the tax department, for revenue collection from the sector. The authorities also recognize the need to enhance public financial management and will implement a five-pillar action plan to this end, as laid out in paragraph 27 of their MEFP.

On the expenditure side, the authorities are committed to implement a prudent policy, with a wage bill capped at 6.7 percent of GDP over the medium term, while maintaining the policy of standardizing public sector wages of central government and provinces in 2010 . This will be attained by enhancing the computerized payroll procedure and rationalizing civil service personnel. Both domestically- and foreign-financed investment will double as a percentage of GDP over the course of the program on account of large investments in infrastructure.

In implementing their fiscal policy, my authorities will put emphasis on creating fiscal space needed to meet the country's poverty reduction objectives, notably by transferring the constitutionallymandated 40 percent of domestic revenue to provinces, which become responsible for the implementation of education, health and rural sector development.

\section{Monetary and exchange policies}

The goal of monetary policy remains price stability under a floating exchange rate regime, which has served the economy well as a buffer to external shocks. To make monetary policy more effective, the $\mathrm{BCC}$ will strengthen its policy framework by introducing more flexibility in the indirect monetary policy instruments (open market operations and reserve requirements) and enhancing its liquidity management and forecasting capacity. It will also improve its communication strategy to better signal policies to market participants. The central bank will intervene in foreign exchange market only to smooth out exchange rate fluctuations.

\section{Financial sector policies}

The authorities will strive to enhance the BCC's operational capacity and put it on a sound financial footing through restructuring and recapitalization, with technical assistance from the IMF. They have adopted an action plan to improve governance and strengthen the central bank's operational 
effectiveness. The recapitalization of the Bank, to be provided by the government, is expected in 2011.

Regarding the banking sector, the authorities aim at safeguarding financial system stability and soundness through an improved regulatory framework, including increased minimum capital requirements for banks and more rigorous licensing procedures; enhanced supervision notably human capacity; strengthened on- and off-site inspection; and macro-prudential functions.

\section{Other structural reforms}

Other structural measures will contribute to reforming the civil service, with the view to streamline it and make it more efficient, and improving economic governance and the business climate to promote private sector-led growth, as detailed in paragraphs 32-36 of the authorities' MEFP. In the same vein, the authorities intend to accelerate the reforms of public enterprises to improve the delivery of services indispensable for boosting growth. In this regard, the government plans to divest from all public enterprises with commercial activities, towards their privatization.

\section{SHORT-TERM POLICIES AND REFORMS}

My authorities recognize that the immediate risks to macroeconomic stability brought about by the current challenging environment ought to be addressed by tightening fiscal and monetary policies and promoting structural and sectoral reforms to pave the way for a viable private sector and for high and lasting economic growth. Already, on the fiscal policy front, they have cancelled all expenditure commitments up to year-end for which goods and services have not been delivered, and postponed the repayment of part of the arrears accumulated in 2007-08, pending their audit.

As regards monetary and exchange policies, the $\mathrm{BCC}$ will pursue the sales of central bank bills, in close collaboration with the Treasury, to mop up excess liquidity in the economy. Exchange market intervention will aim at smoothing unwarranted exchange rate volatility and potentially mopping up excess liquidity.

In the structural reform area, the agenda throughout June 2010 includes the promulgation of a new organic public finance law, adoption of a modern procurement code, streamlining duties and taxes, rationalization of tax exemptions, the continuing restructuring of the $\mathrm{BCC}$, the modernization of the central bank's monetary policy instruments, and the submission to Parliament of a new statistics law.

\section{Debt sustainability and the HIPC process}

The updated debt sustainability analysis conducted jointly by Fund and World Bank staffs show that the DRC remains in debt distress. Absent debt relief under the HIPC Initiative and MDRI, its external debt burden indicators are projected to remain above the policy-dependent thresholds over a protracted periods even with a strong recovery over the medium term. As debt distress continues to exert a drag on the economy, the DRC is in dire need to reach the HIPC completion point as soon as possible. The authorities expect to meet all the completion point requirements, including the trigger points, in 2010, in order to benefit from substantial debt relief and allocate freed domestic resources and additional external support to their poverty reduction and reconstruction efforts. 
Furthermore, even with the HIPC/MDRI debt relief, the country's external outlook would remain vulnerable to adverse shocks. Therefore, the authorities fully understand the need, on their part, to maintain a prudent debt management policy. After amending an important cooperation agreement aimed at building and modernizing infrastructure, while revitalizing the mining sector, to make it consistent with program-related concessionality requirements and with debt sustainability, they recently reiterated their commitment to refrain from contracting or guaranteeing new debt that would jeopardize debt sustainability. They also plan to improve the business climate and enhance legal protection for investments, as indicated earlier. Moreover, my DRC authorities maintain close communication with the Paris Club on the implementation of their economic program, and intend to request from these creditors rescheduling agreements to alleviate the country's debt service burden. They also maintain dialogue with London Club private creditors, with the view to obtain debt restructuring on comparable terms and plan to normalize relations with all bilateral and commercial creditors. In turn, the DRC authorities commit to avoid accumulating any new arrears on nonreschedulable external debt during the program period.

\section{Conclusion}

As a post-conflict country, the Democratic Republic of the Congo now faces an opportunity to consolidate peace and political stability as well as make significant advances towards reconstruction, reducing poverty and meeting the MDGs. As hope is reemerging in DRC, with renewed efforts towards reconciliation and peace in a context of good regional cooperation, it is critical that the international community provides financial and technical support to allow the DRC to pursue its ambitious policy and reform agenda outlined in the authorities' MEFP and step up development efforts.

The swift involvement of the Fund in 2002 had been instrumental to the progress made between that year and late 2005. Fund assistance this time around, through a new PRGF-supported arrangement in support of the Congolese authorities' ongoing efforts to maintain macroeconomic stability, will enable them to mobilize donor support and attract private investors, thereby helping put the country on a path of robust and sustained growth and lasting poverty reduction. Therefore, my DRC authorities would greatly appreciate the Executive Board's support of their requests for a three-year PRGF arrangement and additional interim assistance under the Enhanced HIPC Initiative. 